\title{
ACOUSTIC MODE COHERENCE \\ IN THE ARCTIC OCEAN
}

\author{
by \\ John J. Polcari \\ B.S. (1977, U.S.N.A.), M.S.E.E. (1983, M.I.T.), O.E. (1983, M.I.T.) \\ SUBMITTED IN FARTLAL FULFILLMENT \\ OF THE REQUIREMENTS FOR THE DEGREE OF \\ DOCTOR OF SCIENCE
}

at the

\section{MASSACHUSETTS INSTITUTE OF TECHNOLOGY \\ and the}

\section{WOODS HOLE OCEANOGRAPHIC INSTITUTION}

May, 1986

(c) John J. Polcari, 1986

The author hereby grants M.I.T., W.H.O.I., and the U. S. Government permission to produce and to distribute copies of this thesis document in whole or in part.

Signature of Author

J6int Program in Oceanographic Engineering Massachusetts Institute of Technology and Woods Hole Oceanographic Institution

Certified by

Dr. A.B. Baggeroer, Professor of Ocean Engineering and Electrical Enginetring Thesis Supervisor

Accepted by

William D. Cirant, Chairman, Joint Conmittee for Oceanographic Engineering 


\begin{abstract}
The dual issues of modal decomposition for tonal sound fields and the temporal coherence of the modal amplitudes are investigated for the case of the central Arctic sound channel at very low frequencies $(15-80 \mathrm{~Hz})$. A detailed study of the Arctic modal structure for these frequencies reveals the central role played by the strong Arctic surface duct. The performance of each of four different moclal beamforming algorithms when applied to the vertical array deployed during the FRAM IV Arctic Acoustic Experiment is analyzed. A multiple beam (or decoupled beam) least squares processor produces the most acceptable results for Arctic conditions. The modal decomposition is sensitive to vertical array tilt caused by hydrodynamic drag; a technique for its estimation from the acoustic data is developed.

Tonal data taken from both the horizontal and vertical arrays deployed during FRAM IV is analyzed. Horizontal array results confirm the modal amplitudes generated from vertical array data. The rough surface scattering from the ice canopy places an upper limit of $40 \mathrm{~Hz}$ on efficient surface duct propagation. Attenuation measurements for the first mode show excellent agreement with predictions made for ice scattering using the method of small perturbations and experimental ice statistics. The high levels of coherence observed $(0.95$ to 0.99$)$ show that tonal signal propagation in the Arctic channel is essentially deterministic for time periods well in excess of one hour. The various modes may then be considered to maintain a constant phase relationship over time.
\end{abstract}




\section{Acknowledgements}

A number of people have contributed to the successful conclusion of this thesis, and for their help I am extremely grateful. My thesis advisor. Professor Arthur Baggeroer, has provided both the technical guidance and the moral support that I so badly needed at various times through this effort. Most importantly, I wish to thank him for his boundless patience during what has often seemed an endless task. My thesis committee, consisting of Professors Ira Dyer, George Frisk, Jim Lynch, and Peter Mikhalevsky, has provided me a potent reserve of technical expertise that has significantly broadened my understanding by providing new insights and perspectives on the problems encountered. I consider the conversations I have held with all five of these men to be perhaps the most valuable thing I take with me as I leave the Joint Program.

I am deeply indebted to the U.S. Navy for providing me both the opportunity and the means to complete this degree. In particular, I wish to thank Captain Clark Graham and Commander Dave Whiddon for their unfailing support and their expert help in balancing Navy requirements with educational opportunities. Their patience in what has often seemed a frustrating and drawn out process has also been of critical importance.

I would like to thank the various WHOI personnel with whom I have dealt with for their help in conquering the great number of technical problems associated with the reduction and display of the data. It has been a distinct pleasure to work closely with Eddie Scheer, who has created much of the signal processing and data display software used here (as well as playing a mean piano). Kieth Von Der Heydt. and Nan Galbraith have been most helpful in keeping a rather cranky computer 
system up and running as needed.

Doctor Fred DiNapoli of NUSC, New London, has spent a great amount of time with me carefully reviewing the results and conclusions of this thesis post mortem, an exercise that I have found to be invaluable, particularly for its insight into how this work affects the larger picture of sound propagation in the Arctic Ocean.

My discussions with fellow students have been enlightening, refreshing. and motivating. Once again, I consider these to be a very important part of the "doctoral experience". Josko Catapovic, Peter Dahl, Peter Stein, and Nick Makris have all provided excellent sounding boards for various ideas and speculations. Mike Wengrovitz, (now Professor) Greg Duckworth, Rajan, Steve Petri, and Jiang: Sheng-li have also provided useful comments.

To my roommate, Alan Dion, I owe a note of thanks for putting up with me under what have certainly been less than ideal circumstances. Additional thanks go to the various secretaries and staff associated either directly or indirectly with the Joint Program Office, particularly Marilyn Staruch, Mary Athanis, and Denise Cormier, who have been instrumental in helping to overcome various logistical obstacles encountered along the way. Finally, I wish to thank the person (who, as requested, shall remain anonymous) that invested such a great deal of time and effort to help proof and edit the final version of this thesis. 


\section{Table of Contents}

Abstract $\quad 2$

Acknowledgements $\quad 3$

Table of Contents $\quad 5$

$\begin{array}{lr}\text { List of Figures } & 8\end{array}$

List of Tables $\quad 11$

1. Introduction $\quad 12$

1.1 Motivation 13

1.2 Preliminary Concepts

1.3 Objectives $\quad \underline{24}$

1.4 Contributions $\quad 26$

1.5 Thesis Organization 28

2. A Modal Source Description $\quad 31$

2.1 Normal Mode Propagation in a Range Independent Channel 32

2.2 Normal Mode Propagation in a Range Varying Channel 40

2.3 Modal Source Description at a Far Field Receiving Array

2.4 Summary $\quad 49$

3. The FRAM IV Data Set $\quad 50$

3.1 The FRAM IV Experiment $\quad 50$

3.2 FRAM IV Navigation Data

3.3 Horizontal Azimuth Angle Conventions $\quad 54$

3.4 The TRISTEN Low Frequency Source $\quad \mathbf{5 6}$

3.5 The FRAM IV Receiving Arrays • 58

3.6 The Effects of Hydrophone Sensitivity Mismatches 60

3.7 Sensor Displacement Predictions for the Horizontal and Vertical 61 Arrays

3.8 The FRAM IV Data Acquisition System 66

3.9 Details of the Vertical Array Data Set

3.10 Details of the Horizontal Array Data Set 70

3.11 Preprocessing of the Data $\quad 70$

3.12 An Analysis of the Preprocessed Data 76

3.13 Summary 87

4. The Modal Structure of the Arctic Sound Channel 89

4.1 A Representative Sound Speed Profile from the Central Arctic Ocean $9: 2$

4.2 Temporal and Spatial Sound Speed Variations

4.3 The Acoustic Impact of the Ice Cover 96

4.4 Solutions of the Helmholtz Equation for the Mode Shapes

4.5 An Analysis of the FRAM IV Modal Structure 100

4.5.1 Mode Shape Analysis 
4.5.2 Phase Speed Analysis $\quad 107$

4.5.3 General Observations $\quad 110$

4.6 The Deepest RSR Ray Paths of the TRISTEN/FRAM Channel 113

$\begin{array}{lll}4.7 & \text { Summary } & 113\end{array}$

5. Modal Beamforming: Theory and Simulation 116

5.1 Development of the Least Squares Modal Beamformer 119

5.2 Single Beam versus Multiple Beam Beamformers 123

5.3 Generalizations of the Least Squares Modal Beamformer and the 125 MLM Modal Beamformer

5.4 A Comparison of Modal Beamforming with Plane Wave Beamforming 128

5.5 Estimation of the Sensor Cross Coherence Matrix 130

5.6 Performance of the Various Modal Beamformers 132

5.7 Performance of the Single Beam Least Squares Beamformer 134

5.8 Performance of the MLM Beamformers 142

5.8.1 Performance of the Single Beam MLM Beamformer for 142 Incoherent Modes

5.8.2 The Relationship Between the Single and Multiple Beam 1.47 Variants of the MLM Beamformer for Incoherent Modes

5.8.3 Performance of the Single Beam MLM Algorithm for Vertical 151 Arrays in the Presence of Coherent Interference

5.8.4 Performance of the Multiple Beam MLM Algorithm for Vertical 15z Arrays in the Presence of Coherent Interference

5.8.5 Alternative Implementations of the MLM Algorithm in the 160 Presence of Coherent Interference

5.8.6 Performance of MLM Algorithms for Horizontal Arrays in the 161 Presence of Coherent Interference

5.9 Performance of the Multiple Beam Least Squares Beamformer 16:

5.10 Determination of the Number of Modes to Model 170

5.11 Summary 175

6. The Array Tilt Problem 178

6.1 A Simplified Model for the Shape of the Vertical Array 180

6.2 The Sensitivity of Modal Beamforming to Array Tilt 184

6.3 Examples of the Array Tilt Sensitivity in the Processing 191

6.4 The Array Tilt Accuracy Required for Valid Modal Estimates 193

6.5 The Effect of Tilt on Horizontal Arrays 197

6.6 Recovery of Vertical Array Tilts from the Acoustic Data 198

6.7 Summary 203

7. Data Presentation and Analysis 207

7.1 General Comments 209

7.2 Presentation of the Horizontal Array Processing Results 211

7.2.1 Azimuthal Beamforming 211

7.2.2 Vertical Beamforming 213

7.2.3 Summary of the Horizontal Array Processing Results 2.21

7.2.4 Verification of the Modal Structure 2:22

7.3 Presentation of the Vertical Array Processing Results

7.3.1 The Sensitivity of Vertical Array Data to Array Tilt 2:5 
7.3.2 Determination of Vertical Array Tilt Angles $\quad 229$

7.3.3 Time Series of the Modal Amplitudes $\quad \mathbf{2 3 6}$

7.3.4 Possible Array Movements Seen in the Vertical Array Data 2:38

7.4 Modal Amplitude Analysis $\quad 24:$

7.4.1 Comparison of First Mode Amplitude Estimates from the 243 Horizontal and Vertical Arrays

7.4.2 Modal Attenuation Coefficient Measurements 247

7.4.3 Comparison with Shot Data $\quad \mathbf{2 5 4}$

7.5 Mode Coherence Measurements $\quad 256$

7.6 Relative Phase Measurements for the Vertical Array Data

$\begin{array}{lll}7.7 & \text { Summary } & \mathbf{2 6 0}\end{array}$

8. Summary and Conclusions $\quad 266$

$\begin{array}{ll}8.1 \text { Summary } & 266\end{array}$

8.2 Conclusions 271

8.2.1 Signal Processing Conclusions 271

8.2.2 Conclusions Concerning the Practical Use of Vertical Arrays 275

8.2.3 Underwater Acoustics Conclusions 276

$\begin{array}{ll}8.3 \text { Some Thoughts on Further Work } & 280\end{array}$

Appendices $\quad \mathbf{2 8 3}$

A. On the Inclusion of Signal Components in Generalized Least Squares 28.3

Weighting Matrices

B. The Performance Relationship Between Single Beam and Multiple 286 Beam MLM Algorithms 


\section{List of Figures}

Figure 1-1: Signal Replication for Hydrophones in a Horizontal Array (0 to $80 \mathrm{~Hz}$ Band)

Figure 1-2: The Effect of Modal Structure on the Signals Received by Different Hydrophones in a Vertical Array (0 to $80 \mathrm{~Hz}$ Band)

Figure 2-1: An Idealized Range-Independent Oceanic Waveguide

Figure 2-2: Range-Independent Modal Propagation

Figure 2-3: An Idealized Range-Dependent Oceanic Waveguide

Figure 2-4: Range-Dependent Adiabatic Modal Propagation

Figure 2-5: Relationship of Source and Receiver Coordinate Systems (top view)

Figure 3-1: General Locations of the FRAM and TRISTEN Ice Camps 51

Figure 3-2: Drift of the FRAM and TRISTEN Ice Camps

Figure 3-3: Source Levels for the TRISTEN Source 56

Figure 3-4: Arrangement of the FRAM Receiving Arrays

Figure 3-5: Two Models for the FRAM Vertical Array Shape (second 64 model taken from [85])

Figure 3-6: The FRAM IV Digital Recording System 6 7

Figure 3-7: The Preprocessing Scheme

Figure 3-8: Parks-McClellan FIR Filter Frequency Response 72

Figure 3-9: Preprocessing Output Time Series - Vertical Array Sensor it Magnitudes at $47.00 \mathrm{~Hz}$

Figure 3-10: Preprocessing Output Time Series - Vertical Array Sensor T Phases at $47.00 \mathrm{~Hz}$

Figure 3-11: Preprocessing Output Time Series - Vertical Array Sensor Magnitudes at $17.75 \mathrm{~Hz}$

Figure 3-12: Preprocessing Output Time Series - Vertical Array Sensor 84 Phases at $17.75 \mathrm{~Hz}$

Figure 4-1: Sound Speed Profile of the Water Column at the Receiving 91 Arrays

Figure 4-2: Assumed Bottom Characteristics at the Receiving Arrays 03

Figure 4-3: Historical Bathymetry along the TRISTEN/FR.AM 9-4 Propagation Path (taken from [14])

Figure 4-4: Shape of the First Mode at Various Frequencies

Figure 4-5: Shape of the Second Mode at Various Frequencies

100

Figure 4-6: Shape of the Third Mode at Various Frequencies

101

Figure 4-7: Shape of the Fourth Mode at Various Frequencies

$10 \%$

$10: 3$

Figure 4-8: Mode Shape Levels at $91 \mathrm{~m}$ for Various Frequencies 105

Figure 4-8: Relative Advantage of the First Mode over the Next Four 106 Modes at $91 \mathrm{~m}$ versus Frequency

Figure 4-10: Horizontal Phase Speeds of the First Three Modes versus 108 Frequency

Figure 4-11: Modified Sound Speed Profiles Used as Limiting Cases for 10 ? the Central Arctic Profile 
Figure 4-12: Deepest RSR Ray for the TRISTEN/FRAM Transmission 112 Path

Figure 5-1: Theoretical Modal Sidelobe Pattern - $30 \mathrm{~Hz}$ Single Beam Least Squares Beamformer for 10 Modes (all 18 elements)

Figure 5-2: Theoretical Modal Sidelobe Pattern - $47 \mathrm{~Hz}$ Single Beam Least Squares Beamformer for 45 Modes (all 18 elements)

Figure 5-3: Theoretical Modal Sidelobe Pattern - $47 \mathrm{~Hz}$ Single Beam Least Squares Beamformer for 45 Modes (top 9 elements only)

Figure 5-4: Theoretical Modal Sidelobe Pattern - $47 \mathrm{~Hz}$ Single Beam Least Squares Beamformer for 45 Modes (every other element only)

Figure 5-5: Theoretical Modal Sidelobe Pattern - $30 \mathrm{~Hz}$ Single Beam MLM Beamformer for 28 Modes (all 18 elements)

Figure 5-6: Output of Single Beam MLM Beamformer versus Time for Incoherent Synthetic Data at $30 \mathrm{~Hz}$

Figure 5-7: Output of Single Beam MLM Eeamformer versus Time for Incoherent Synthetic Data at $47 \mathrm{~Hz}$

Figure 5-8: Output of Single Beam MLM Beamformer versus Time for Coherent Synthetic Data at $47 \mathrm{~Hz}$

Figure 5-9: Components of a Coherent Sound Field for a Notional 16: Horizontal Array

Figure 5-10: Output of Multiple Beam Least Squares Beamformer versus Time for Coherent Synthetic Data at $47 \mathrm{~Hz}(7$ modes included in beamformer)

Figure 5-11: Output of Single Beam Least Squares Beamformer versus 168 Time for Coherent Synthetic Data at $47 \mathrm{~Hz}$ ( 7 modes shown)

Figure 5-12: Output of Multiple Beam Least Squares Beamformer versus Time for Coherent Synthetic Data at $47 \mathrm{~Hz} / 8$ modes included in beamformer)

Figure 5-13: Singularity Coefficient versus Mode Count at $15 \mathrm{~Hz}, 30 \mathrm{~Hz}, 172$ and $47 \mathrm{~Hz}$

Figure 6-1: Modal Beamforming for an Untilted Array in a Simple 18; Channel

Figure 6-2: Modal Beamforming for a Tilted Array in a Simple Channel 186

Figure 6-3: Sensitivity of Vertical Array Modal Beamforming to Array 188 Tilt (Synthetic Data at $30 \mathrm{~Hz}$ - First Realization)

Figure 6-4: Sensitivity of Vertical Array Modal Beamforming to Array Ix! Tilt (Synthetic Data at $30 \mathrm{~Hz}$ - Second Realization)

Figure 6-5: Sensitivity of Vertical Array Modal Beamforming to Array 190 Tilt (Synthetic Data at $\mathbf{4 7} \mathrm{Hz}$ )

Figure 6-6: Horizontal Phase Speed for the DC Mode versus Frequency 19:;

Figure 6-7: Normalized Residual Error as a Function of Tilt Angle for 200 Synthetic Data at $47 \mathrm{~Hz}$ (No Excluded Modes Present)

Figure 6-8: Normalized Residual Error as a Function of Tilt Angle for 201 Synthetic Data at $47 \mathrm{~Hz}$ (Excluded Modes Present)

Figure 7-1: Output of Single Beam MLM Beamformer Scanned in 21: Azimuth for $27.00 \mathrm{~Hz}$ Tone (Horizontal Array) 
Figure 7-2: Output of Single Beam MLM Beamformer Scanned in Phase Speed for $27.00 \mathrm{~Hz}$ Tone (Horizontal Array)

Figure 7-3: Output of Single Beam Least Squares Beamformer Scanned in Phase Speed for $27.00 \mathrm{~Hz}$ Tone (Horizontal Array)

Figure 7-4: Output of Single Beam MLM Beamformer Scanned in Phase Speed for $17.75 \mathrm{~Hz}$ Tone (Horizontal Array)

Figure 7-5: Output of Single Beam MLM Beamformer Scanned in Phase Speed for $17.75 \mathrm{~Hz}$ Tone (Horizontal Array with 3 Sensors Excluded)

Figure 7-6: Output of Single Beam MLM Beamformer Scanned in Phase Speed for $47.00 \mathrm{~Hz}$ Tone (Horizontal Array)

Figure 7-7: First Mode Phase Speed versus Frequency; Comparison of Horizontal Array Observations with Theory

Figure 7-8: Conceptual Relationship of the Different Types of Vertical Array Processing Outputs

Figure 7-9: Output of Multiple Beam Least Squares Modal Beamformer versus Array Tilt for $17.75 \mathrm{~Hz}$ Tone (Vertical Array)

Figure 7-10: Output of Multiple Beam Least Squares Modal Beamformer versus Array Tilt for $47.00 \mathrm{~Hz}$ Tone (Vertical Array)

Figure 7-11: Residual Error of Multiple Beam Least Squares Modal Beamformer versus Array Tilt for $17.75 \mathrm{~Hz}$ Tone (Vertical Array with 3 Modes Included)

Figure 7-12: Residual Error of Multiple Beam Least Squares Modal Beamformer versus Array Tilt for $47.00 \mathrm{~Hz}$ Tone (Vertical Array with 7 Modes Included)

Figure 7-13: Output of Multiple Beam Least Squares Beamformer versus Time for $17.75 \mathrm{~Hz}$ Tone (Vertical Array)

Figure 7-14: Residual Error of Multiple Beam Least Squares Modal Beamformer versus Array Tilt for $23.50 \mathrm{~Hz}$ Tone (Vertical Array with 4 Modes Included)

Figure 7-15: Output of Multiple Beam Least Squares Beamformer versus Time for $23.50 \mathrm{~Hz}$ Tone (Vertical Array)

Figure 7-16: Summary of First Mode Amplitude Measurements

Figure 7-17: Summary of Second Mode Amplitude Measurements

Figure 7-18: Summary of Third Mode Amplitude Measurements

Figure 7-19: First Mode Attenuation - Experimental and Predicted

Figure 7-20: Second Mode Attenuation - Experimental and Predicted

Figure 7-21: Third Mode Attenuation - Experimental and Predicted

Figure 7-22: Sonogram of Explosive Shot Transmitted Over the TRISTEN/FRAM C'hannel as Received on $90 \mathrm{~m}$ Hydrophone of the Vertical Array (300 ft Source Depth)

Figure 7-23: Time Series of the Relative Phases of Various Mlode Pairs :58

for $17.75 \mathrm{~Hz}$ Data 


\section{List of Tables}

Table 3-I: TRISTEN/FRAM Navigation Data for the Horizontal Array 5.1 Data Set

Table 3-II: TRISTEN/FRAM Navigation Data for the Vertical Array 5.5 Data Set

Table 3-III: The FRAM IV Vertical Array Recording Configuration 68

Table 3-IV: The FRAM IV Vertical Array Data Set 69

Table 3-V: The FRAM IV Horizontal Array Data Set 70

Table 3-VI: FIR Filter Characteristics

Table 4-I: Number of Modes with Turning Points above $3800 \mathrm{~m}$ and 101 $2500 \mathrm{~m}$

Table 5-I: Modes Resolvable by Multiple Beam Least Squares Modal 17t Beamformer

Table 6-I: Results of Legendre Polynomial Analysis Results for the 18:3 NRL Model of the FRAM IV Vertical Array Shape

Table 6-II: Tilt Angle Accuracy Requirements for the FRAM IV 196 Vertical Array

Table 7-I: Results of the Array Tilt Estimation Procedure

Table 7-II: Mode Coherence Estimates for the Vertical Array Data

Table 7-III: Relative Phase Estimates for the Vertical Array Data $\underline{2} 60$

Table 7-IV: Comparison of Measured and Expected Relative Mode 260 Phases for $17.75 \mathrm{~Hz}$ Data 


\section{Chapter 1}

\section{Introduction}

It is the spatial structure in a wave field that carries the great majority of what might be called the immediate information about the surrounding environment. Exploitation of this structure can provide answers to questions of object existence, location, identification, and arrangement. Making instrumentation sensitive to the spatial structure of a wave field is accomplished by providing the detection system with a directional response. While it is possible to build detectors having inherent directionality, it is often easier to synthesize the desired directional response through the employment of arrays of simpler detectors. The outputs of these detectors are then combined, usually in a linear fashion, through a process known as beamforming.

The art and science of beamforming has a rich history of application. The human body employs the concept by incorporating pairs of both eyes and ears. The fundamental physics of the technique is similar to that of such diverse areas of science as diffraction grating theory, lens optics, $x$-ray crystallography, and radio antennae design. The underlying principles of beamforming are applicable whenever one is dealing with either the directional transmission of energy or the directional reception of energy propagating in a wave field.

This thesis deals with a fairly new and unique method of applying the principles of beamforming in the complex vertical structure exhibited by the low frequency acoustic field found in the world's oceans. Since the electromagnetic spectrum encounters unacceptable levels of attenuation in seawater, the acoustic spectrum is the wave field of choice for all oceanic sciences. In particular, the very 
lowest frequency acoustic waves (those below a few hundred Hertz) are (apable of propagating efficiently over hundreds of kilometers in water, making them a prime candidate for use in long range ocean surveillance and communication applications. An understanding of the beamforming techniques appropriate for use with this wave field is a central engineering issue in all but the simplest efforts to use it both scientifically and practically.

\subsection{Motivation}

Traditional acoustic beamforming theories typically consider the detection for generation) of plane waves in an unbounded fluid medium. The primary properties of the medium that impact sound transmission (its sound speed and density) are typically assumed to be constant throughout the medium. The constant medium assumption is made to avoid an overly complicated propagation problem, while the plane wave assumption is attractive for two reasons. First, it is physically realistic in the case of a true unbounded uniform medium, since the spherical wave generated by a point source may be considered to be locally planar at long ranges from the source. Second, it produces mathematically tractable results, since, once the assumption is made, the beamforming problem can be interpreted in terms of spatial Fourier transforms of the observed sound field. A large body of theory and experience involving Fourier techniques can then be borrowed from other disciplines.

The underwater acoustic research of the last two decades has increasingly pointed towards the conclusion that an unbounded uniform medium is a poor choice of model for the world's oceans. This is particularly true at the low frequencies of interest here, where even the deepest ocean depths may correspond to only a few hundred acoustic wavelengths. This conclusion is well understood within the sound 
propagation community, where more appropriate layered waveguide models have gained wide acceptance. However, its impact on beamforming theory has been considerably more limited. This is primarily because the incorporation of a nonuniform medium in beamforming theory makes the plane wave assumption physically unrealistic; it is the propagating normal modes that are the fundamental spatial sound structures in a waveguide. Giving up the plane wave assumption in turn requires that the Fourier transform interpretation and its attendiant mathematical elegance be abandoned. Instead, the traditional techniques have generally been extended through the assumption of a medium that is locally uniform in the vicinity of the array. The plane wave assumption may then be maintained: the resultant beamformer output is interpreted in terms of the spatial Fourier transform of the sound field present at the array.

This approach has three advantages. First, the procedure is reasonably robust. Second, it is well understood, from both the theoretical and application viewpoints. Third, it still works well for horizontal arrays in oceanic waveguides, since, for this particular geometry, the vertical structure of the normal modes is effectively hidden; for a horizontal array, each mode appears to. be no more than a plane wave with a particular grazing angle.

The technique also has a number of serious drawbacks. First, it is not very insightful, since, as mentioned earlier, the modal decomposition is much more physically relevant in a waveguide than the Fourier decomposition. Second, it is not a particularly efficient estimation scheme, for it requires a large number of parameters the amplitudes and phases of the incoming plane wares from all possible directions) to be estimated in order to characterize the total sound field at the array. Representations that minimize the number of parameters needed to completely describe the field generally make better estimation techniques because 
each parameter's estimate includes at least a small amount of error. Finally. and most importantly, the approach obscures the impact that the waveguide model ('an have on the issue of target parameter estimation.

Consider the information about a point source that an array can extract from the sound field in a uniform unbounded medium. The direction to the source (its three-dimensional bearing) is easily found by estimating the direction of the signal's wavenumber vector, but an estimate of target range can only be made through the measurement of the curvature of the spherical wave. At anything other than short ranges from the source, this implies an impractically long array, so that range estimation is generally not considered feasible. This limitation on target range estimation is closely linked to the unbounded medium assumption; if one adopts a layered waveguide model, though, direct estimation of the source range (and depth) from the observed sound field is at least theoretically possible for an array of finite aperture deployed vertically across the waveguide. Source range information can be obtained most directly from the relative phases of the various propagating modes, while the source depth can be extracted from the relative modal amplitudes. Other. less direct (and perhaps more robust), target range and depth estimation techni(jues are also possible.

The great potential value of source range and depth estimation serves as the motivation for studying the nature of the modal amplitudes and phases. One of the fundamental scientific issues that must be addressed in assessing the practical utility of range estimation in a waveguide is whether or not the relative modal amplitude's and phases are temporally stable. If both types of parameter can be considered to be constant over reasonable lengths of time, then there is some chance that range estimation techniques might be feasible; conversely, if one or both show significant random behavior, then the chance for practical success is small. Note that it is the 
stability of relative modal amplitudes and phases that is of interest here; it is entirely possible for these parameters to be essentially constant even if the equivalent absolute measurements display a great deal of random behavior. All that is required is that the absolute measurements not be independently random.

The ultimate purpose of this thesis is to study the temporal stability of relative modal amplitudes and phases in one specific instance; that of long range sound propagation in the central Arctic Ocean. There are a number of reasons for choosing to study the Arctic channel. First, a reasonably extensive data set, including vertical array data, is available from the FRAM IV Arctic Acoustic Experiment. Second, the unique nature of the Arctic simplifies investigation in some important respects, although it complicates the effort in others. Finally, the Arctic channel possesses characteristics that make it a prime candidate for successful application of the range and depth estimation techniques of interest.

Intimately related to any such attempt is, of course, the ability to estimate the modal amplitudes and phases directly, which is equivalent to the development of beamformers more appropriate to the waveguide nature of the low frequency ocean acoustic channel. In addition to supporting the ultimate objective of the thesis. these are of obvious interest in their own right. An ancillary purpose of this thesis is to characterize some of the different ways in which the modal decomposition might be implemented and to study the performance of these methods in the Arctic sound channel. 


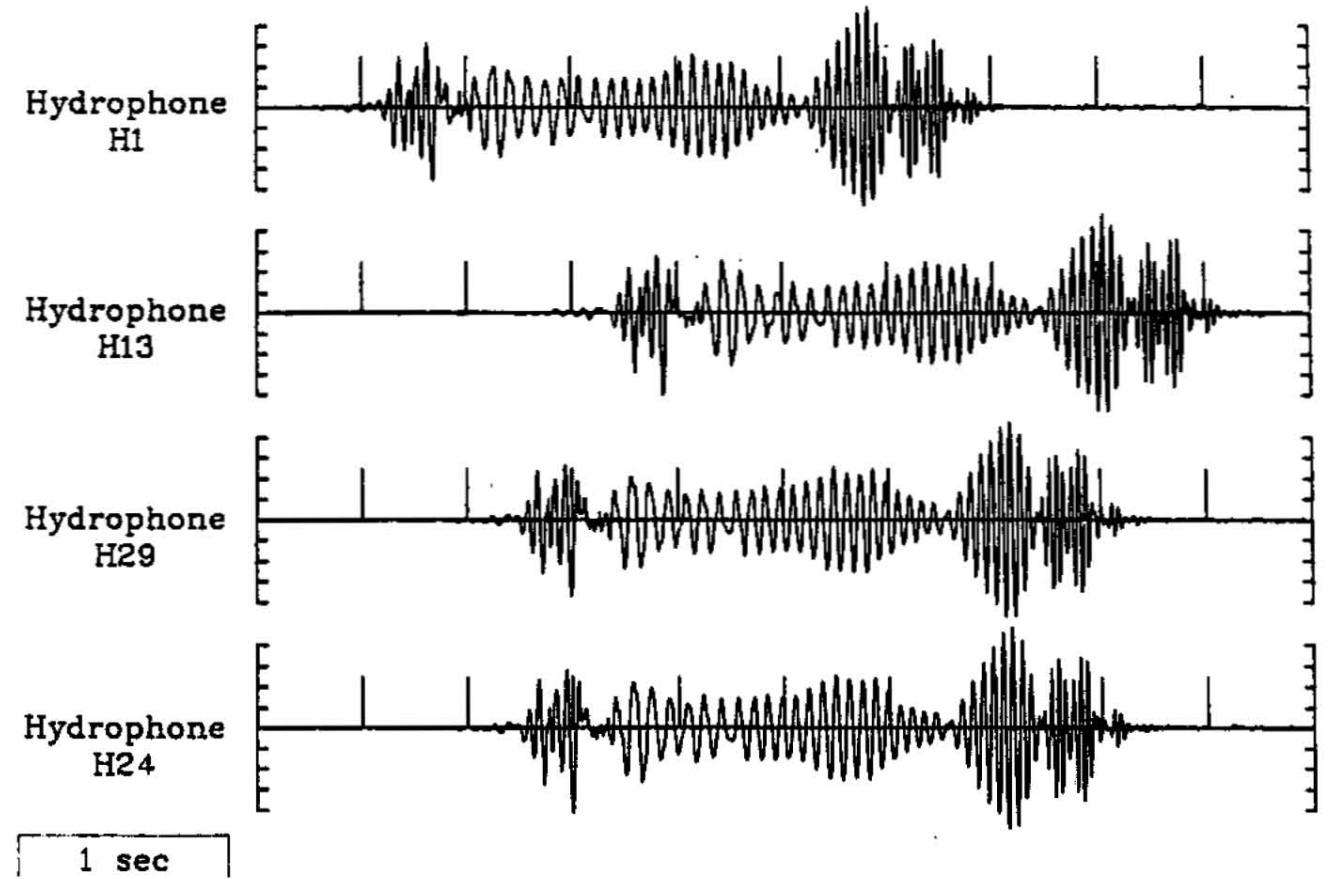

Figure 1-1: Signal Replication for Hydrophones in a Horizontal Array (0 to $80 \mathrm{~Hz}$ Band)

\subsection{Preliminary Concepts}

Consider the set of time series displayed in Figure 1-1. These traces are the signals received on various hydrophones of a horizontal array from an explosive source several hundred kilometers away. The important observation to make is that the signal received on any sensor can be considered to be just a time shifted replica of the signal received on any other sensor. Most linear beamforming techniques, at some point in their derivation, assume that the effect demonstrated in Figure 1-1 is true, whether or not it actually holds in practice.

Because traditional beamforming techniques are so dependent on the shifted replica assumption, the methods experience difficulty when employed in situations 
where the the assumption is not valid, as is the case with the set of time series shown in Figure 1-2. These traces are the signals received from the same explosive source as in the previous figure, but now taken from various elements in a vertical array, so that the elements are distributed above and below each other in the water column rather than to either side, as was the case in Figure 1-1. Vertical arrays are of natural interest in a waveguide, since they sample the modal structure of the sound propagation much more fully than do horizontal arrays. Figure 1-2, for example, clearly highlights the modal structure of the channel much better than Figure 1-1. In particular, the long coda for the signal is the arrival of the first mode, which travels with a slower group velocity and with more dispersion than the other modes. The gradual shortening of the coda with depth demonstrates quite dramatically the variation of the shape of the first mode with frequency. Since the propagation path for the signals of Figures 1-1 and 1-2 is the same central Arctic channel examined throughout this thesis, the reader will find the actual shape of the first mode for various frequencies in Figure 4-4.

If it is the modal propagation structure that is to be studied, as it is here, then the shifted replica assumption is clearly inappropriate. A review of the channel model assumed by traditional beamforming techniques provides a better understanding of the alternatives. This model may be partially described in the vernacular of signal processing as the known signal in noise model. This means that the sound energy of interest, called the signal, is considered to be a known. deterministic waveform, with some unknown but non-random parameters that do not vary over time. The sound energy not of interest is called the noise, and is assumed to be an additive random process. A tonal signal of known operating frequency typically has two unknown parameters, its amplitude and its absolute phase; these can be incorporated into a single unknown complex amplitude if. the 


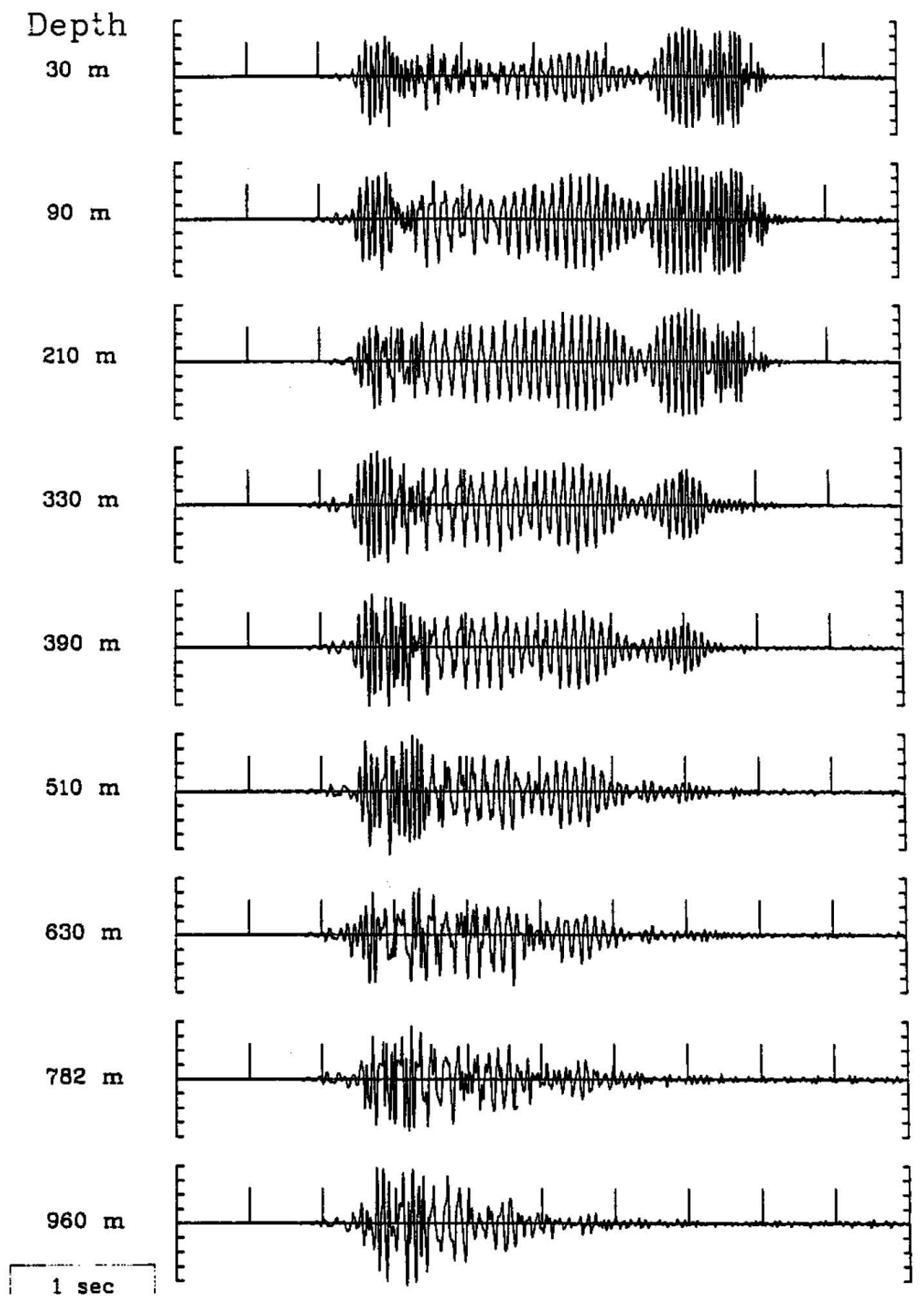

Figure 1-2: The Effect of Modal Structure on the Signals Received by Different Hydrophones in a Vertical Array (0 to $80 \mathrm{~Hz}$ Band) 
complex exponential notation is used. If one first quadrature demodulates the received signal at the nominal tonal frequency and then performs a low pass filtering operation, then the resultant output for any single hydrophone may be written as

$$
p(t)=A+n(t)
$$

where $p(t)$ is the complex demodulated form of the received signal, $A$ is the unknown complex amplitude, and $n(t)$ is the complex noise process that results from processing the noise process received at the hydrophone through the demodulator and filter.

This description tells only part of the full story, however, since it includes no information about the spatial nature of the field; that is, it does not indicate how the signal at one hydrophone is related to the signal at some other hydrophone located nearby. The shifted replica assumption is used to define this relationship. The model of equation (1.1) can then to be extended to cover all $N$ sensors in the array. Grouping all the different demodulated hydrophone outputs into one $N \times 1$ complex vector, and their noise processes into another, one may write

$$
\mathbf{p}(t)=\underline{\mathbf{E}} A+\underline{\mathbf{n}}(t),
$$

where the $N \times 1$ complex steering vector $\underline{\mathbf{E}}$ indicates the phase shift that each replica undergoes in propagating to its particular hydrophone

$$
\underline{\mathbf{E}}=\left[\begin{array}{c}
e^{j \theta_{1}} \\
e^{j \theta_{2}} \\
\dot{\vdots} \\
e^{j \theta_{N}}
\end{array}\right]
$$

When the sensors in the array are distributed at varying depths in a 
waveguide, as they are in Figure 1-2, the modal nature of the propagation must be acknowledged, making the simple shifted replica assumption an inadequate description. Instead, each normal mode of the total field can be considered to be an independent waveform, so that the the shifted replica assumption must be applied on a mode by mode basis rather than to the full signal. A vector of unknown complex amplitudes now exist, one for each of the $M$ modes included in the model. Note that the selection of the number of modes $M$ to consider is essentially a modeling decision that must be made by the user. The mathematical signial description $\underline{\mathbf{E}}$ now becomes an $N \times M$ complex steering matrix of the form

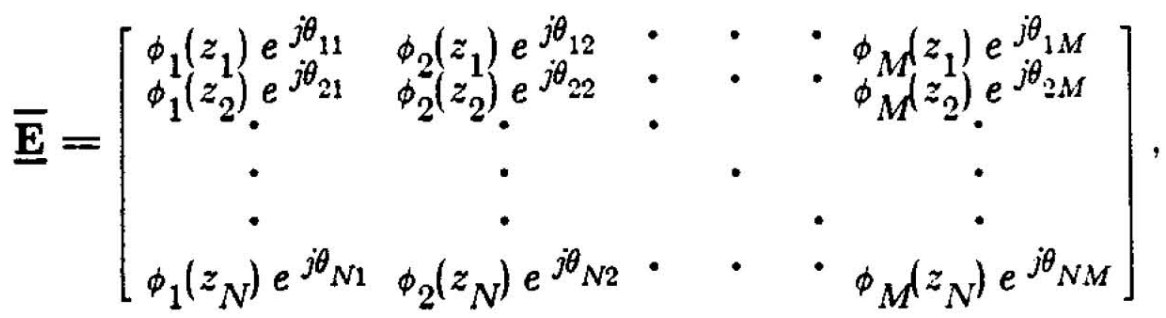

where the $k^{\text {th }}$ column of the matrix represents the generalized steering vector for the $k^{\text {th }}$ mode, which now includes amplitude factors $\phi_{k}\left(z_{i}\right)$ that reflect the size of the mode shape at the various sensor depths in addition to the modal phase shifts $\theta_{i k}$. The resulting model is then

$$
\underline{\mathbf{p}}(t)=\overline{\mathbf{E}} \underline{\mathbf{A}}+\underline{\mathbf{n}}(t),
$$

where $\underline{\mathbf{A}}$ is the $M \times 1$ vector of unknown complex modal amplitudes. In Chapter 2 , this model is derived rigorously from both range-independent and rangedependent normal mode descriptions.

The model implied by equation (1.5) forms the basis for all the different modal beamforming algorithms developed in Chapter 5. In its temporal aspects. this model is similar to the one used in the plane wave beamforming development. The two differ only in their spatial aspects, and then only in the number, and not the 
type, of the unknown signal parameters.

Even though they have been modeled here as non-random constants, it is reasonable to assume that the complex modal amplitudes will actually vary randomly over time in any real ocean. The size and structure of these fluctuations, particularly the size and structure of the phase fluctuations for the different modes. is of fundamental importance to the proper characterization of the acoustic propagation from a signal processing viewpoint. Indeed, the measurement and analysis of these fluctuations is the ultimate objective of this thesis. The eritical issue here is whether or not the phase variations found for any one mode are independent of the phase variations found for the other modes. If the fluctuations occur independently of one another, then they destroy the phase relationship between the various modes; in this case the modes are said to be incoherent with respect to each other. On the other hand, if any fluctuations that occur do so simultaneously across all the modes, then the modes remain phase locked with respect to each other, and may be considered to be coherent.

Examination of the coherence of the different modes is important for a number of reasons, three of which are mentioned here. First, the issue affects propagation modeling. If the modes are incoherent with respect to each other, then the individual modal phases can be ignored, and the total field energy should be computed by summing individual modal energies. Conversely, if the modes are coherent, then both their amplitudes and their relative phases have to be predicted accurately, since they are needed to properly compute the total field. Second, the issue is central to the question of the feasibility of direct target range estimation, which, as discussed earlier, requires a strongly coherent mode field in order to produce accurate results. Finally, from a signal processing point of view, a measure of the mode coherence is a critical element in any complete description of the multi- 
path environment of the channel. An incoherent mode field indicates that the various transmission paths are independent and phase random; the often used WSSUS assumption (wide sense stationary, uncorrelated scatterers) implicitly assumes this characterization. On the other hand, a consideration of the linear relationship between rays and modes leads to the conclusion that the multiple paths cannot be considered independent if the mode field remains coherent.

A good measure of mode coherence can be made from the $M \times M$ modal cross-coherence matrix, which exists in two versions. The unnormalized form of the matrix can be defined as

$$
\overline{\mathbf{S}}_{\mathrm{M}}=\mathrm{E}\left[\underline{\mathbf{A}}^{+}\right]
$$

where the + symbol stands for the conjugate transpose operation. The notation E [.] nominally represents the average over an ensemble of different trials; however, time averages rather than ensemble averages are almost always used when implementing the process. Each element of the matrix can then be written as

$$
\overline{\mathrm{S}}_{\mathrm{Mik}}=\mathrm{E}\left[A_{i} A_{k}^{*}\right]=\mathrm{E}\left[\left|A_{i}\right|\left|A_{k}\right| e^{j\left(\psi_{i}-\psi_{k}\right)}\right] \text {, }
$$

where

$$
A_{i}=\left|A_{i}\right| e^{j \psi_{i}}
$$

is the magnitude-phase representation of the complex modal amplitude. The elements of the normalized modal cross-coherence matrix are scaled versions of the unnormalized matrix elements, namely,

$$
\overline{\mathbf{T}}_{\mathrm{M} i k}=\frac{\mathrm{E}\left[A_{i} A_{k}^{*}\right]}{\sqrt{\mathrm{E}\left[\left|A_{i}\right|^{2}\right] \mathrm{E}\left[\left|A_{k}\right|^{2}\right]}}
$$

The normalized matrix has two useful properties: its diagonal elements are all unity; and the magnitude of any non-diagonal element is not larger than 1.0. 
To understand how the cross-coherence matrix reflects the phase randomness of the various modes, consider a situation where the magnitudes of the modal amplitudes are constant and only the modal phases are allowed to be random. Then the elements of the normalized modal cross-coherence matrix assume the form

$$
\overline{\mathbf{T}}_{\mathrm{Mik}}=\mathrm{E}\left[e^{\jmath\left(\psi_{i}-\psi_{k}\right)}\right] .
$$

If the two random phases are now completely independent of each other, then their difference is also completely random, so that the expectation takes on a value of zero. On the other hand, if the two modes are phase locked, then the phase difference takes on a constant value, even though the individual phases themselves may be totally random; the resulting magnitude of the matrix term is one. The magnitude of any term of the normalized modal cross-coherence matrix, therefore, provides a quantitative measure of the coherence of the two modes comprising the term.

Two issues remain; that of estimating the modal amplitudes and their crosscoherence based on the model of equation (1.5), which is exactly the modal beamforming problem; and that of the applying a model that assumes a non-random signal to mode coherence estimation, which is a measure of the signal's randomness. These subjects are taken up in Chapter 5.

\subsection{Objectives}

The ultimate purpose of this thesis is to measure the coherence between different normal modes emanating from a single tonal source and then propagating through the central Arctic acoustic sound channel to ranges of several hundred kilometers. Such an investigation implies a great deal more than that which is explicitly stated. To begin with, if one wishes to measure the properties of the 
normal modes, then one must first understand the modal structure of the sound channel of interest; this, in turn, requires thorough investigation of some of the oceanographic properties of the local environment, including items such as the channel sound speed profile and surface and bottom descriptions. Obviously, there is a need to understand in detail the nature of the available data and the equipment and techniques used to obtain it. The received signal energy must be separated into the component modes while at the same time rejecting as much background noise as possible. This effort turns out to be non-trivial, and much of this thesis is spent understanding the methods available, their performance in various situations, and their sensitivity to the realities of field research.

If one has managed to accomplish all of the above, then there is a reasonable chance of making some valid measurements of the coherence of the various modes. At the same time, greater insight is (hopefully) gained into the nature of sound transmission in the channel under consideration, and thus it is valuable to consider the acoustic propagation implications of the results.

This thesis thus has five general objectives:

1. To describe and understand the modal structure of the central Arctic sound channel encountered during the FRAM IV Experiment, including the effect of different environmental aspects on this structure and its implications for the source and receiver geometries involved;

2. To develop the beamforming methods needed to make direct modal amplitude estimates for narrowband tonal signals, particularly when vertical arrays are employed, and to answer some of the performance questions regarding these techniques;

3. To assess the operational utility of vertical arrays, and to understand the impact of some of the practical realities encountered in their use, particularly with respect to the modal decomposition process;

4. To answer the question of whether or not the amplitudes of the various modes generated from from a single harmonic point source remain 
coherent after propagation to long ranges in the Arctic sound channel; and

5. To assess the implications of the modal amplitude and coherence estimates made in light of the current understanding of central Arctic sound channel propagation.

\subsection{Contributions}

This thesis provides a number of significant contributions to the fields of underwater acoustics and signal processing. These contributions may be classified into three areas.

First, there are several contributions made to the discipline of signal processing and, in particular, to beamforming theory. The modal beamforming algorithms developed in Chapter 5 are not really new. Hinich [42], [43], Clay [17]. and Bucker [9], among others, have all covered similar ground. In any case, the results are straightforward analogs of earlier plane wave processing results examined by such a large number of other investigators, such as Schweppe [74], Capon [10]. and Baggeroer [2]. Rather, it is the performance evaluation of these estimates which is unique in this work, especially because it is made for a real array and a set of modes developed from a real channel. The assessment of mode resolution provides some valuable insight into which modes can be distinguished and which cannot. The study of the numerical stability limits to the number of modes that can be simultaneously included in a multiple beam beamformer contributes in a similar way. The performance relationships of multiple beam and single beam algorithms, especially for the MLM approach, is a subject not adequately addressed previously. Finally, the examination of the difficulties of the MLM algorithm in the face of coherent modal signals provides a different perspective on another important signal processing issue that is all too often incorrectly ignored. 
-27-

The second area of contribution deals with the practical issues involved in the use of vertical arrays. Most important here is the finding that modal decomposition is extremely sensitive to array tilt. This result has tremendous operational significance, because it indicates that the shapes of vertical arrays must be known far better than they are presently, if the devices are to be exploited to anything near their theoretical potential.

The area in which this thesis makes the largest number of significant contributions, however, is to the discipline of underwater acoustics, particularly to the understanding of how low frequency sound propagates to long ranges in the central Arctic Ocean. This thesis represents the first time that modal amplitude and phase measurements have been made for low frequency tonal signals using actual field data from a vertical array and direct modal decomposition techniques. Previous efforts involving modal amplitude estimation for tonal sources, such as the shallow water experiment conducted by Ferris [33] and the laboratory investigation of Hobaek, Tindle, and Muir [44], have all allowed the channel itself to accomplish the modal separation by utilizing pulsed sinusoids which then separate temporally into the various modal arrivals because of different modal group velocities. The present effort obviously has much wider scientific and practical application.

Beyond the intrinsic value of modal beamforming as a tool for further scientific investigation, additional contributions are made in understanding the process of low frequency transmission in the central Arctic. In particular, both the modal amplitude and coherence estimates made here are the first of their kind to be attempted, and provide some very new and very different insights into Arctic Ocean propagation. The picture of an essentially deterministic sound field that emerges from the coherence measurements and the stability observations not only verifies Mikhalevsky's earlier findings [57], but also expands on them considerably, since the 
various propagation paths have now been at least partially separated. The classification of two significantly different propagation regimes, one below $40 \mathrm{~Hz}$ and the other above, has practical implications beyond its usefulness in cataloging Arctic propagation; these are discussed in the conclusions of Chapter 8 . The ice scattering effects displayed in the results provide new insight into a very important problem that has not been satisfactorily solved. Of the most interest here are the indications that sound energy scattered by the ice remains coherent with the specular field, and may play a significant role in the overall sound transmission picture. Finally, the in depth study of the modal structure of the central Arctic conducted in Chapter 4 provides new perspective on the role that the strong Arctic surface duct plays in channel propagation.

\subsection{Thesis Organization}

The remainder of this thesis consists of seven chapters and two appendices. In general, each chapter covers one major aspect of the overall analysis.

Chapter 2 lays the theoretical groundwork for modal beamforming techniques by providing a mathematical description of the sound field generated at a distant array by a point source that is located in a waveguide. This description, which is based on acoustic normal mode propagation theory, is then couched in a form to which optimal estimation theory can easily be applied.

Chapter 3 provides a full description of the FRAM IV Arctic Acoustic Experiment from which the data set of interest is drawn. Most of the important technical details of the experiment (navigation data, hardware descriptions, etc.) are also provided. A preliminary analysis of some of the practical issues involved in dealing with suspended array systems is included. This is done to highlight the issues' relative importance to the modal beamforming problem when using both 
horizontal and vertical arrays. The preprocessing scheme used to compress the data set to a usable size is discussed. Finally, some of the resultant time series are analyzed to provide preliminary insight into the modal energy distributions and coherence values that might be expected.

Chapter 4 presents a detailed analysis of the significant environmental aspects of the central Arctic sound channel. An in depth study of the modal structure encountered during the FRAM IV Experiment is made for the source-receiver geometries of interest. This study is a necessary preliminary to any modal beamforming effort, since it defines the specific spatial structures of interest throughout the rest of the thesis.

In Chapter 5, several different modal beamforming algorithms are developed theoretically from optimal estimation theory. Their theoretical performance is then analyzed, leading to the selection of a multiple beam least squares technique as the most suitable method for processing the data from the vertical array.

Chapter 6 studies the most important practical issue that arises when attempting modal beamforming with vertical arrays; that of array tilt. The modal decomposition process is shown to be extremely sensitive to the effective tilt angle. Since no direct tilt measurements were made during the FRAM IV experiment, a relatively simple method for its estimation from the acoustic data is developed.

Chapter 7 presents the outputs of the modal beamforming processor when applied to the actual data set. Horizontal and vertical array measurements are contrasted, and both are compared against theoretical predictions. Some corroborating shot data is also presented. The modal amplitude and mode coherence results are analyzed to understand their implications for central Arctic sound propagation. 
Chapter 8 provides a summary of the results and a general discussion of the final conclusions of the thesis.

Each of the two appendices deals with a particular mathematical aspect of modal beamforming theory needed to make the analysis rigorous. 


\section{Chapter 2}

\section{A Modal Source Description}

The purpose of this chapter is to rigorously develop, in a form that is tractable within the context of signal processing theory, a mathematical model to describe the sound pressure field that a harmonic point source imbedded in a waveguide generates at a distant receiving array. The final result of this development has already been presented (in a somewhat simplified form) in equations (1.4) and (1.5). The present chapter is designed to provide a more detailed presentation of the implied assumptions and the line of reasoning that leads to this result. The development consists of two parts. First, normal mode propagation theory is reviewed for both the range independent and range dependent channels. Normal mode theory is the natural starting point for this thesis, since it is modal characteristics that are of interest here. Besides providing a solid link from the various forms of normal mode theory to the present effort, the review also provides an opportunity to introduce the nomenclature and notation that is used throughout the rest of the work.

The remainder of this chapter is concerned with the conversion of the various modal propagation theories to forms that describe the sound pressure field in the immediate vicinity of a distant receiving array. This conversion is important, as it represents the boundary between theories in the underwater acoustics domain and those of the signal processing domain. The results then become the basis for all further work in this thesis. The assumptions and approximations needed to complete the conversion are carefully presented, and the change in notation to the matrix forms typically used in signal processing is described. 
In its simplest form, that of a hard or non-propagating bottom, range independent normal mode theory is a simple application of the standard separation of variables approach to the solution of the wave equation. Pekeris, in his classic paper [64], first showed how the concept could be extended to the case of a more realistic ocean bottom, that of an infinite fluid half space with a given sound speed and density. Today, of course, any number of standard references include discussions on the subject [79], [18]. Range dependent normal mode theory is somewhat more recent and not as well documented. Originally proposed by Pierce [65] and Milder [58], it is an area of active interest that has been used to investigate the effect of range variations in the surface, in the water column, and in the bottom. Examples of recent work include Rutherford [72], Dozier and Tappert [27], [28], and Beilis and Tappert [5].

\subsection{Normal Mode Propagation in a Range Independent Channel}

Consider the channel illustrated in Figure 2-1. An idealized oceanic waveguide is assumed to be horizontally stratified and symmetric with respect to the angle dimension of a cylindrical coordinate system, making only the range and depth dimensions of interest. The waveguide is of depth $H$ with boundaries consisting of a pressure release surface at $z=0$ and a bottom at $z=H$. The bottom may be either hard (non-propagating) or soft (propagating), but the former is assumed for ease of development. A discussion of the propagating bottom development is presented in the sequel. An arbitrary sound speed variation that is a function of depth, but not of time or range, is assumed. For simplicity, a density which is constant over depth is assumed; the approach can be easily extended to include densities that vary with depth.

Let a harmonic point source of frequency $f_{0}$ be located at $(r, z)=\left(0, z_{s}\right)$. The 


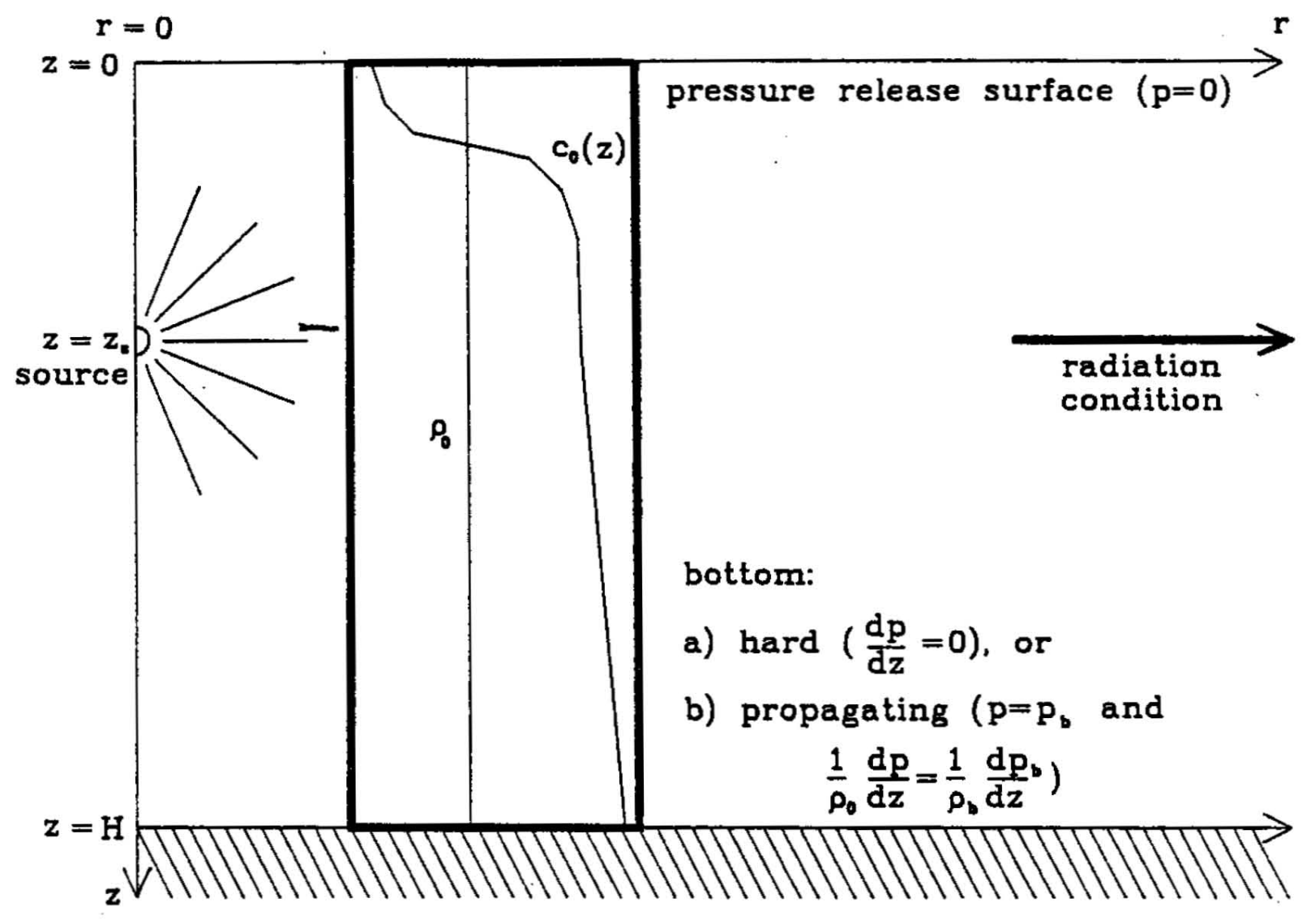

Figure 2-1: An Idealized Range-Independent Oceanic Waveguide

wave equation for the sound pressure may be written as

$$
\frac{1}{r} \frac{\partial}{\partial r}\left(r \frac{\partial p}{\partial r}\right)+\frac{\partial^{2} p}{\partial z^{2}}-\frac{1}{c_{0}^{2}(z)} \frac{\partial^{2} p}{\partial t^{2}}=-j 2 S e^{j 2 \pi \int_{0} t} \frac{\delta(r)}{r} \delta\left(z-z_{s}\right)
$$

where

$$
p=p(r, z, t)
$$

and where $S$ is the sound pressure level in $\mu \mathrm{Pa}$ at a distance of 1 meter from the source in an infinite uniform medium (the units of $S$ are $\mu \mathrm{Pa} \cdot \mathrm{m}$ ). The factor of 2 arises from the different normalizations of the impulse function in the cylindrical 
and spherical coordinate systems. The appropriate boundary conditions are

$$
\begin{aligned}
& p(r, 0, t)=0, \\
& \left.\frac{\partial p}{\partial z}\right|_{r, H, t}=0,
\end{aligned}
$$

and a radiation condition at infinite range.

Taking the temporal Fourier transform of equation (2.1) and evaluating it at the frequency of interest yields the Helmholtz equation

$$
\frac{1}{r} \frac{\partial}{\partial r}\left(r \frac{\partial \mathrm{p}}{\partial r}\right)+\frac{\partial^{2} \mathrm{p}}{\partial z^{2}}+\left(\frac{2 \pi f_{0}}{c_{0}(z)}\right)^{2} \mathrm{p}=-j 2 S \frac{\delta(r)}{r} \delta\left(z-z_{s}\right),
$$

where

$$
\mathrm{p}=\mathrm{p}\left(r, z, f_{0}\right)=\left.\mathbf{F}[p(r, z, t)]\right|_{f=f_{0}}
$$

Range independent normal mode theory for the hard bottom case then arises as a natural separation of variables solution to equation (2.5). The depth functions $p_{i}(z)$ which provide the various mode shapes are the eigenfunctions of the onedimensional Helmholtz equation

$$
\frac{d^{2} \phi_{i}}{d z^{2}}+\left[\left(\frac{2 \pi f_{0}}{c_{0}(z)}\right)^{2}-k_{i}^{2}\right] \phi_{i}=0
$$

with appropriate boundary conditions derived from equations (2.3) and (2.4). The horizontal wavenumbers $k_{i}$ of the various modes are related to the associated eigenvalues. Because of the nature of the boundary conditions, it is easy to cast this problem into a Sturm-Liouville form [41], so that the mode shapes form a complete orthonormal (CON) set. This CON set has three important properties. To begin with, the functions within the set are orthogonal, so that

$$
\int_{0}^{H} \frac{\phi_{i}(z) \phi_{k}(z)}{\rho_{0}} d z=0 \quad \text { for } \quad i \neq k .
$$


The density $\rho_{0}$ is included here in analogy to the results obtained when the density is allowed to vary with depth; for that condition, the reciprocal of the density function acts as the weighting function in the orthogonality relation for the mode shapes of pressure. Note that this differs from the results obtained for the mode shapes of acoustic velocity potential, where the density term appears in the numerator of the orthogonality relation rather than the denominator.

In addition to being orthogonal, the functions are also normalized, so that

$$
\int_{0}^{H} \frac{\phi_{i}^{2}(z)}{\rho_{0}} d z=1 .
$$

Finally, and most importantly, the completeness or closure property guarantees that any arbitrary function of $z$ can be completely represented by an appropriately weighted sum (perhaps infinite) of the various $\phi_{i}(z)$.

Decomposing equation (2.5) on the mode set and using the delta function expansion

$$
\delta\left(z-z_{s}\right)=\sum_{i=1}^{\infty} \frac{\phi_{i}(z) \phi_{i}\left(z_{s}\right)}{\rho_{0}}
$$

leads to the range equation for each mode

$$
\frac{1}{r} \frac{d}{d r}\left(r \frac{d R_{i}}{d r}\right)+k_{i}^{2} R_{i}=-j \frac{2}{\pi} \frac{\delta(r)}{r} .
$$

This is a Bessel equation of order zero, having the two types of Hankel functions as its solution. Such a result is expected, since the range dependence represents the cylindrical spreading of each mode. The radiation condition requires that the Hankel function of the second kind be selected for strictly outward propagation. Note that if the temporal exponential in equation (2.1) were chosen to have the 
opposite sign, the proper choice to meet the radiation condition would be the Hankel function of the first kind. At distances of more than a few wavelengths from the source, the Hankel function may be replaced by its asymptotic equivalent

$$
H_{0}^{(2)}\left(k_{i} r\right) \sim \sqrt{\frac{2}{\pi k_{i} r}} e^{j\left(k_{i} r-\pi / 4\right)}
$$

yielding

$$
p(r, z, t)=\sum_{i} b_{i}(r) \phi_{i}(z) e^{\jmath\left(2 \pi f_{0} t-k_{i} r\right)},
$$

where

$$
b_{i}(r)=\frac{S}{\rho_{0}} \sqrt{\frac{2 \pi}{k_{i} r}} \phi_{i}\left(z_{s}\right) e^{j \pi / 4}
$$

Each term of the result has been conveniently arranged in three parts. $b_{i}(r)$ plays the role of a range dependent modal amplitude, while $\phi_{i}(z)$ provides the normalized depth dependence that represents the shape of the mode and the complex exponential provides the traveling wave phase advance. While the purpose of this development has traditionally been to compute a sound pressure level as a function of depth, the point of interest in this thesis is rather the internal form of each term and, particularly, the result for the modal amplitude given in equation (2.14).

The resulting solution thus provides the following description of the propagation (shown in Figure 2-2):

1. The source excites each mode to a level proportional to the size of the mode shape at the source depth; and

2. Each mode then propagates independently outward from the source as a non-homogeneous cylindrical wave (since the wave amplitude varies with depth) having its own unique wavenumber and depth dependence. 


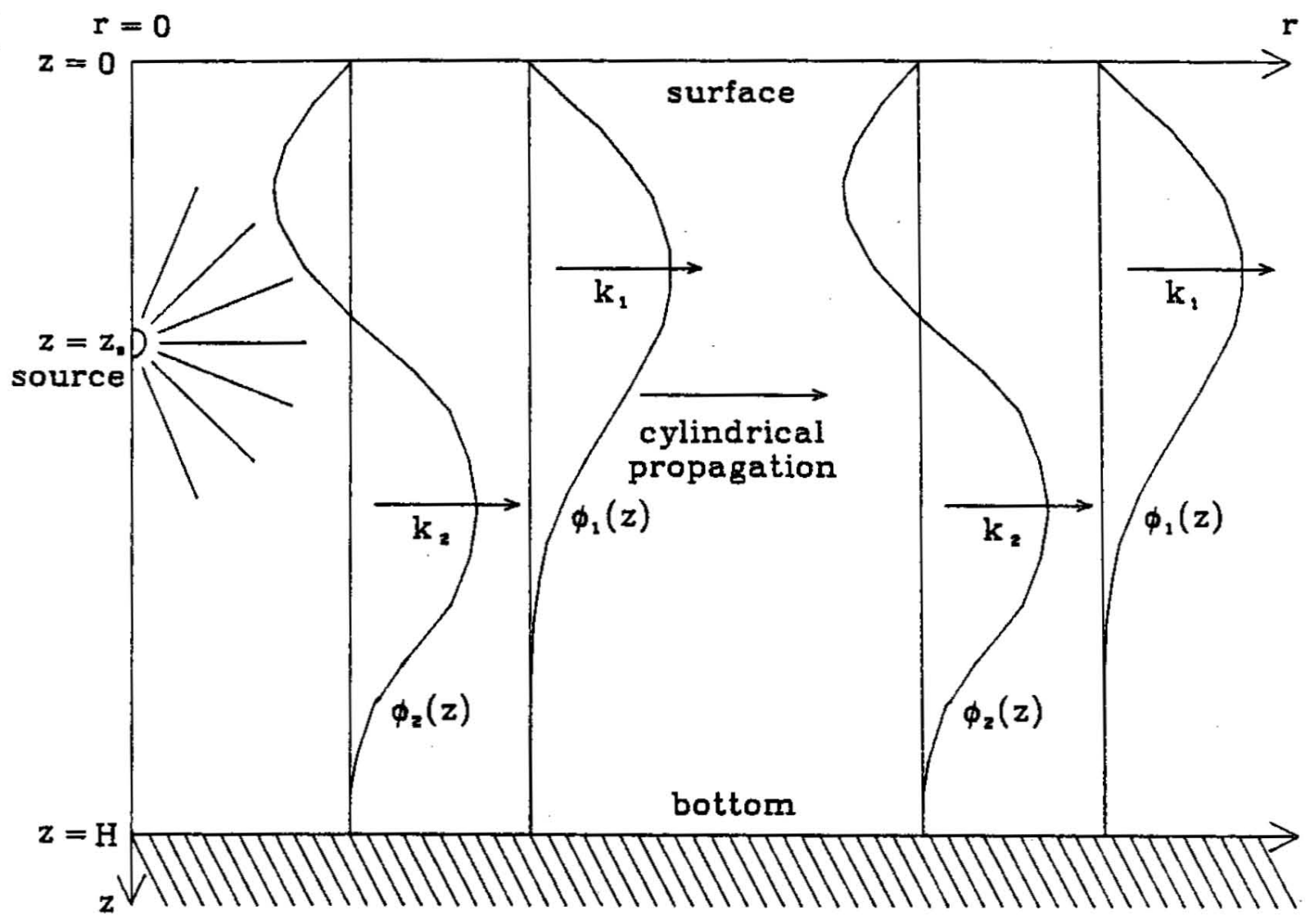

Figure 2-2: Range-Independent Modal Propagation

Although the expression in equation (2.13) nominally extends over an infinite mode set, only a finite number of modes actually propagate. Beyond a certain mode number, the associated modal horizontal wavenumbers turn imaginary, yielding a decaying exponential in range rather than a propagating wave solution. Such modes are known as being in cutoff. At long ranges from the source, the contributions to the sound pressure field from these modes in cutoff become negligible, effectively limiting the sum to a finite (although possibly large) number of modes. The number of modes that need to be included in the sum may be further reduced by other effects on an individual case basis. Two examples are 
selective modai excitation due to the deployment depth of the source and physical attenuation processes which favor some modes over others.

Having completed the development for the condition of a hard bottom, the more physically realistic case of a propagating bottom must now be addressed. A full development for a propagating bottom along the lines of Pekeris [64] has been avoided, as it requires much more mathematical complexity yet generates no significant differences in the final result. Instead, the propagating bottom results are simply contrasted with those presented above to provide some insight into the relationship between the two problems.

The primary difference between the hard bottom condition and the propagating bottom condition occurs in the definition of the CON mode set. The lower boundary condition associated with equation (2.7) in the latter case no longer allows it to be classified as a Sturm-Liouville problem, so that a CON set of mode functions cannot be guaranteed. However, it is still possible to define a set consisting of a finite number of trapped mode shapes. These mode shapes can be made orthonormal through proper scaling, so that equations (2.8) and (2.9) are still applicable if the range of integration is extended to infinity. This is reasonable, since, for an infinite half space bottom, the resulting sound pressure field also extends infinitely in depth.

The set of trapped modes is not complete, however, so that although the results presented in equations $(2.13)$ and $(2.14)$ are applicable to the portion of energy carried in the trapped modes, it is possible for energy not resident in the trapped modes to also contribute to the sound pressure field. This additional contribution is carried by the continuum of modes that propagate into the bottom. and a further term involving an integral over the infinitely dense continuum mode set must be included in equation (2.13) to account for it. The contribution can be 
expected to attenuate with range, however, as more and more of the continuum energy is lost due to propagation into the bottom. At long ranges, the continuum may be neglected, so that equations (2.13) and (2.14) still pertain; but with the sum limited to the trapped modes. Thus, the net effect of the propagating bottom is twofold: to change the range of the summation from the modes not in cutoff to the trapped modes; and to increase the minimum range at which the result is valid.

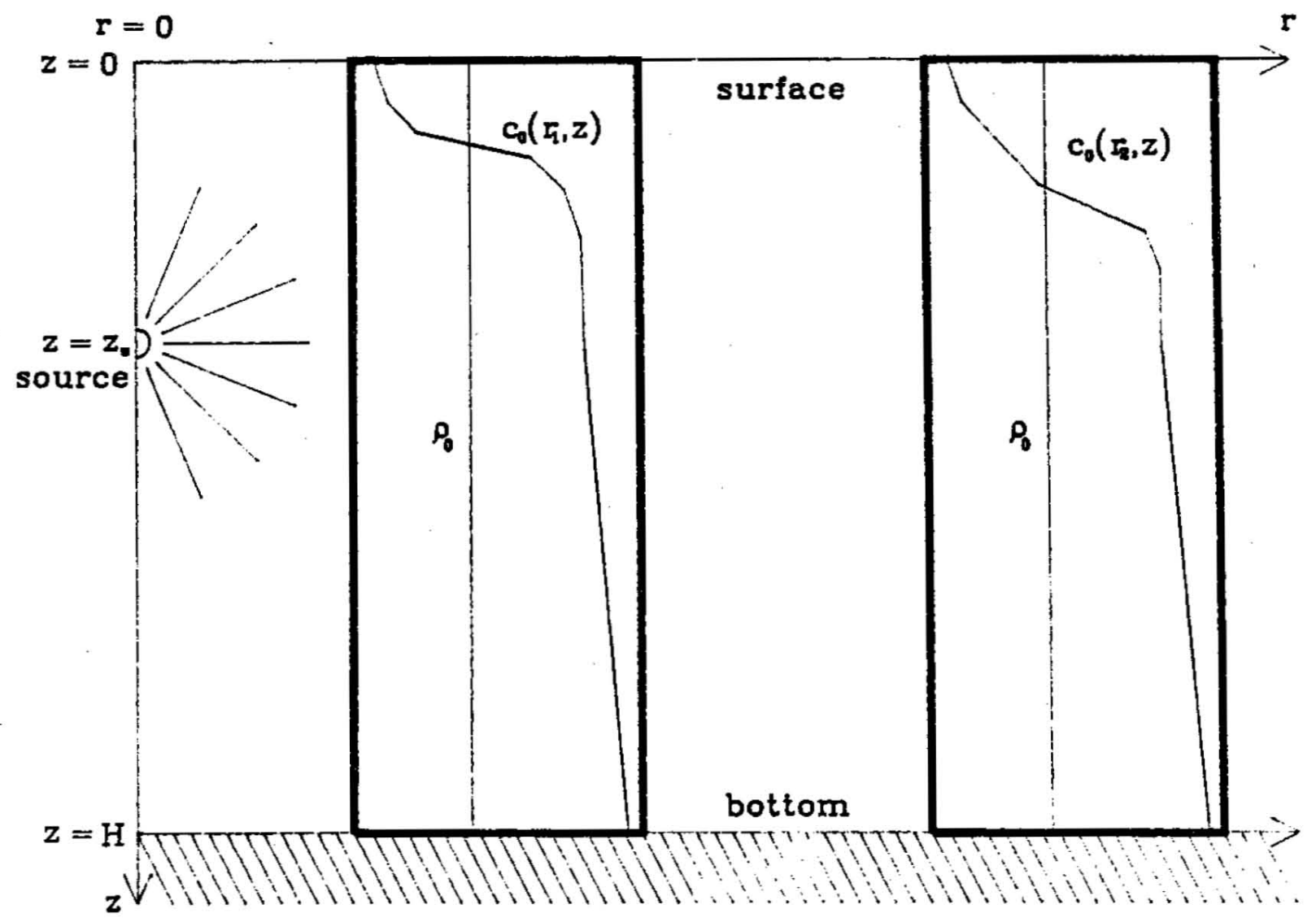

Figure 2-3: An Idealized Range-Dependent Oceanic Waveguide 


\subsection{Normal Mode Propagation in a Range Varying Channel}

The most restrictive assumption made in the previous section is the characterization of the channel properties, including boundary conditions, as being independent of range. To relax this assumption, one can assume a sound speed that is now a function of both depth and range (see Figure 2-3). This change makes the resulting partial differential equation inseparable, so that the approach of the previous section must be abandoned. Instead, a partial separation of variables is invoked by allowing the various mode shapes to vary in range as well. In other words, as the modes propagate outward from the source, their shapes are now allowed to change as they encounter channel range variations. To find the range dependent mode shapes, one must solve the equivalent of the one-dimensional Helmholtz equation (2.7), now parameterized in range

$$
\frac{\partial^{2} \phi_{i}}{\partial z^{2}}+\left[\left(\frac{2 \pi f_{0}}{c_{0}(z, r)}\right)^{2}-k_{i}^{2}(r)\right] \phi_{i}=0,
$$

where

$$
\phi_{i}=\phi_{i}(z, r) .
$$

Suitable boundary conditions must also be included. The approach requires that the associated eigenvalues, and thus the modal horizontal wavenumbers, also be functions of range. If the discussion is again restricted to a hard bottom boundary condition, a CON set of mode shapes can be found at any particular range. Because the separation of variables is only partial, the resulting range equations remain coupled; following Rutherford [72], one obtains 


$$
\begin{aligned}
\frac{1}{r} \frac{d}{d r}\left(r \frac{d R_{i}}{d r}\right) & +k_{i}^{2}(r) R_{i}=-j 2 S_{\phi_{i}}\left(z_{s}, r\right) \frac{\delta(r)}{r} \\
& -\sum_{k}\left[A_{i k}(r)\left(\frac{1}{r} R_{k}+2 \frac{d R_{k}}{d r}\right)+B_{i k}(r) R_{k}\right],
\end{aligned}
$$

where the coupling parameters are

$$
\begin{gathered}
B_{i k}(r)=\frac{2}{k_{i}^{2}(r)-k_{k}^{2}(r)} \int_{0}^{H} \frac{1}{\rho_{0}} \frac{\left(2 \pi f_{0}\right)^{2}}{c_{0}^{3}(z, r)} \frac{\partial c_{0}(z, r)}{\partial r} \phi_{i}(z, r) \phi_{k}(z, r) d z \\
\text { for } i \neq k ; \quad 0 \quad \text { for } \quad i=k ;
\end{gathered}
$$

and

$$
A_{i k}(r)=\frac{d B_{i k}}{d r}-\int_{0}^{H} \frac{1}{\rho_{0}} \frac{\partial \phi_{i}(z, r)}{\partial r} \frac{\partial \phi_{k}(z, r)}{\partial r} d z
$$

The assumptions implicit in the derivation of these coupling parameters are a constant density and range invariant boundary conditions. Allowing the boundary conditions to vary with range affects the form of the coupling coefficients [ 720$]$. However, most problems involving range varying boundary conditions can be recast to have range independent boundaries by the proper redefinition of sound speed and density. The comments made previously concerning the inclusion of soft or propagating bottoms are, in general, also applicable here.

Because the range equations presented in (2.17) are coupled, they are not typically amenable to analytical solution, and further development beyond this point involves either numerical evaluation or the application of approximations. As might be expected, the resulting general solution is not very revealing: 


$$
p(r, z, t)=\sum_{i} R_{i}(r) \phi_{i}(z, r) e^{j 2 \pi \int_{0} t}
$$

One particular approximation generates an important subset of coupled mode theory that is more analytically tractable. Adiabatic mode theory arises by ignoring the effects of the coupling coefficients in (2.17). This is equivalent to assuming only very gradual channel variations in range, which is often the case in practice. Dropping the coupling terms once again leads to independent range equations. In addition, the assumption of gradual range variations requires that, in the large range limit, the solutions of these equations must be asymptotic to

$$
R_{i}(r) \sim b_{i}(r) e^{-j \int_{0}^{r} k_{i}(\epsilon) \epsilon d \epsilon}
$$

The full adiabatic solution is then

$$
p(r, z, t)=\sum_{i} b_{i}(r) \phi_{i}(z, r) e^{\left.\not \lambda 2 \pi f_{0} t-\int_{0}^{r} k_{i}(\epsilon) \epsilon d \epsilon\right)}
$$

Unlike the range independent case, it is not possible to find a general closed form solution for $b_{i}(r)$. The nature of the coefficient preceding the range delta function on the right hand side of equation (2.17) does guarantee that $b_{i}(r)$ are proportional to both the source level $S$ and the size of the mode shape at $r=0$.

Adiabatic mode theory thus generalizes the physical picture of mode propagation as follows (as illustrated in Figure 2-4):

1. The source excites each mode to a level proportional to the size of the mode shape at the source depth, where the mode set is evaluated for the source sound speed profile; and

2. Each mode then propagates independently outward from the source as a 


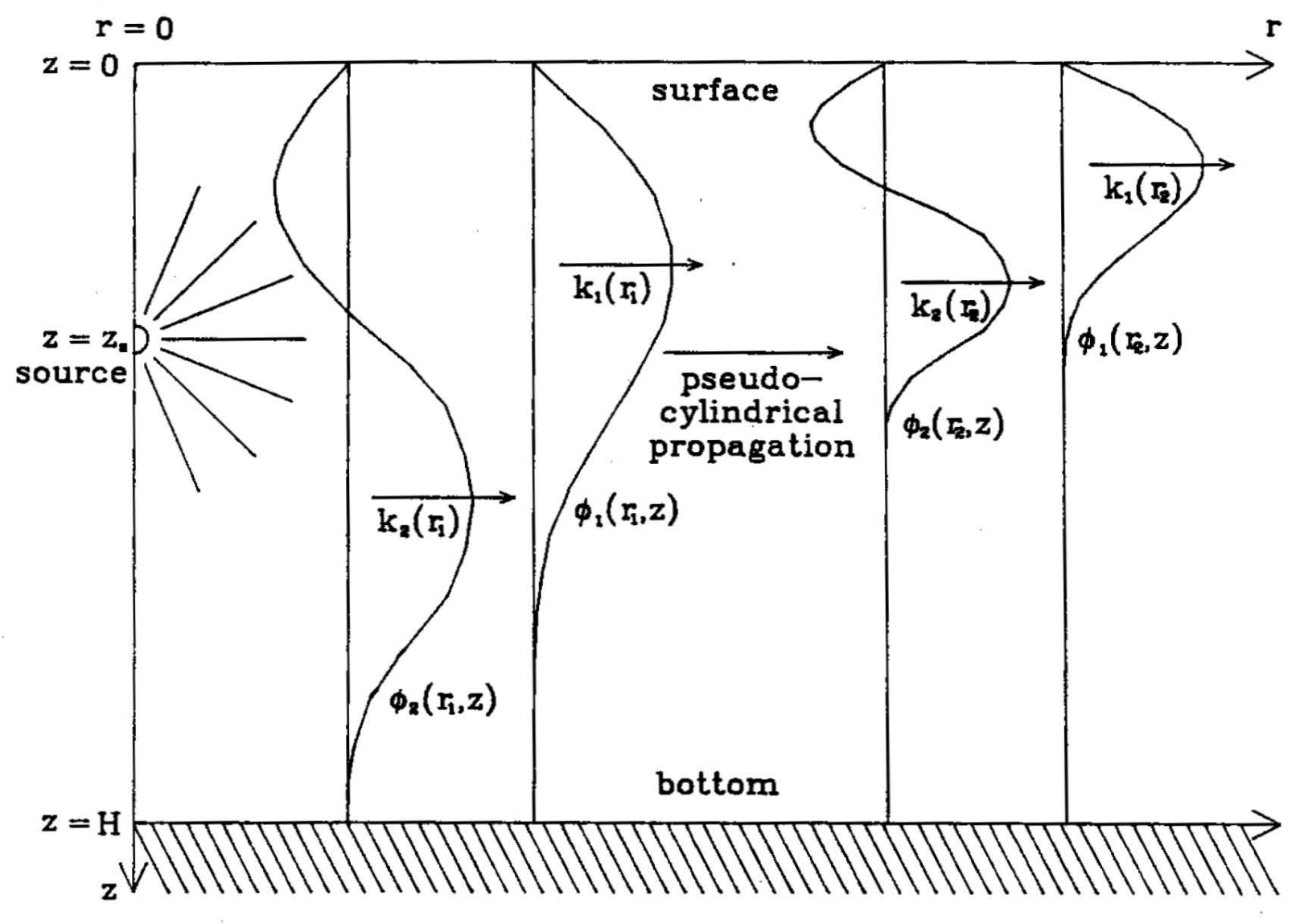

Figure 2-4: Range-Dependent Adiabatic Modal Propagation

non-homogeneous cylindrical wave (since the wave amplitude varies with depth) having its own unique wavenumber and depth dependence. Both of these are now allowed to vary with range. It is important to realize, however, that in this approximation the modes still propagate independently.

Full coupled mode theory further complicates the picture by including mode conversion effects. In this case, the various modes are allowed to exchange energy in addition to varying their shapes and wavenumbers as they propagate down the channel, so that they no longer propagate outward in a completely independent fashion. 


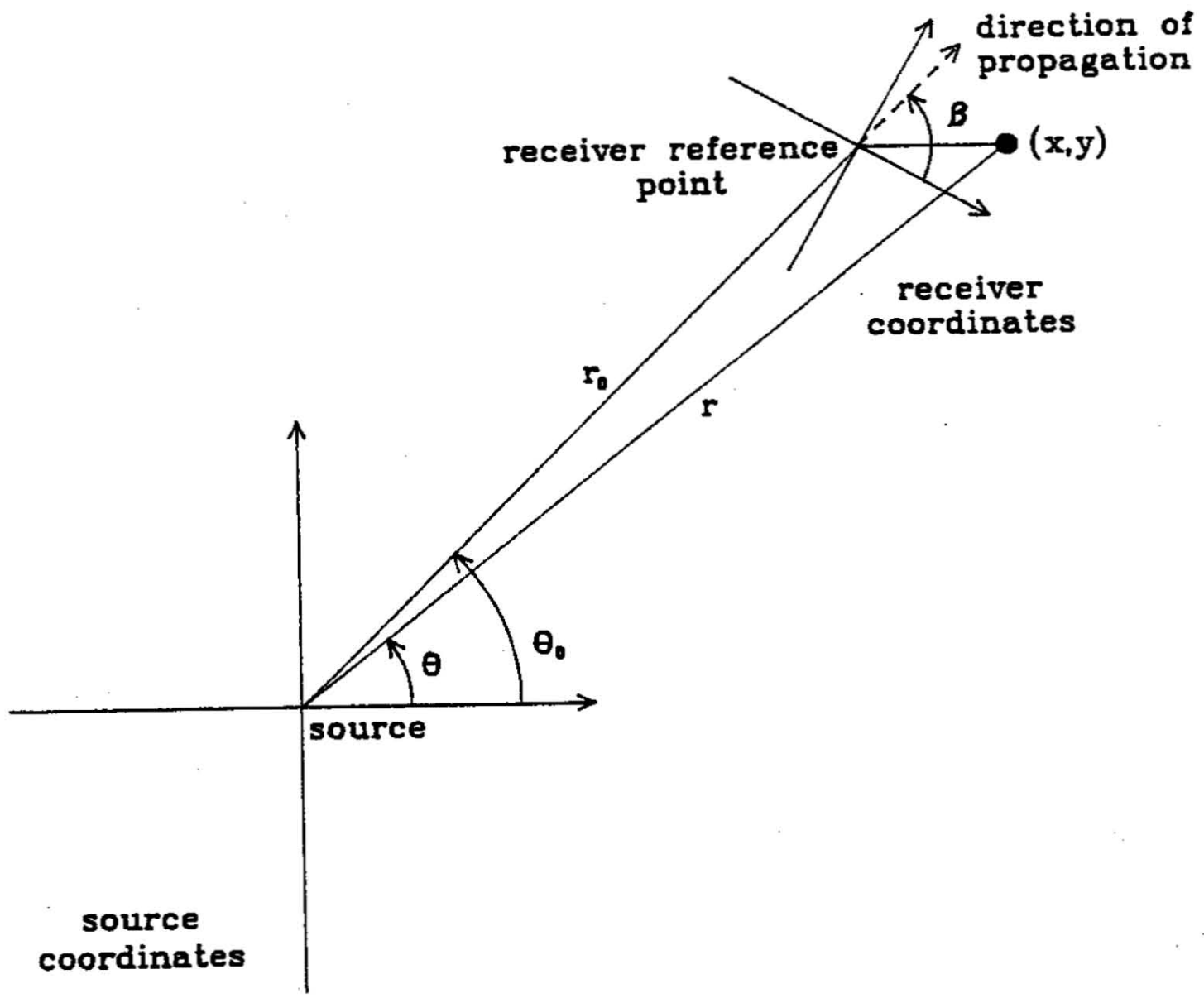

Figure 2-5: Relationship of Source and Receiver Coordinate Systems (top view)

\subsection{Modal Source Description at a Far Field Receiving Array}

While the descriptions developed above are quite useful when dealing with underwater acoustic propagation theory, a further step is necessary to convert them into a form useful in the context of signal processing. Array theory deals with the field at the receiver rather than at the source; therefore, it is appropriate to shift coordinates to a system with its origin at some arbitrarily defined reference point 
near the receiving array, and then to expand the field representation in the vicinity of this new origin. Several approximations and assumptions can then be used to simplify the resulting expressions.

Consider the diagram of the pair of two-dimensional horizontal coordinate systems shown in Figure 2-5. The $(r, \theta)$ coordinate system at the left represents the polar coordinates in which the propagation problem has been solved. The $(x, y)$ coordinate system on the right represents the new receiver coordinates, with an origin at the arbitrarily defined receiver reference point. This point is assumed to be located at range $r_{0}$ and angle $\theta_{0}$ in source coordinates. The angle $\beta$ is the horizontal direction of propagation at the receiver reference point with respect to the receiver coordinate system.

From the law of cosines, the source range $r$ for any point $(x, y)$ in receiver coordinates may be found to be

$$
r=r_{0} \sqrt{1+2\left(x / r_{0}\right) \cos \beta+2\left(y / r_{0}\right) \sin \beta+\left(x / r_{0}\right)^{2}+\left(y / r_{0}\right)^{2}}
$$

In the immediate vicinity of the receiver reference point, the receiver coordinate offsets $x$ and $y$ are small when compared to $r_{0}$. For this condition, the terms containing these offsets are of first order, while the terms involving their squares are of second order. Expanding the square root as a binomial series and dropping all . terms beyond first order leads to

$$
r \approx r_{0}+x \cos \beta+y \sin \beta
$$

Neglecting the higher order terms is equivalent to approximating the cylindrically propagating modes with ones that are planar in the immediate vicinity of the array. In the sequel, the approximation in equation (2.24) is applied to all the sensors in the receiving array. To guarantee that the approximation is valid, two restrictions 
are needed. First, the receiver reference point must be chosen to be close to the array; second, the array aperture must be small compared to the source-receiver range. Both of these conditions are easily met in practice.

Conversion of the range independent modal solution to the new coordinate system simply requires the replacement of $r$ in equations (2.13) and (2.14) with the expression given in (2.24); retaining first order accuracy in the phase but limiting amplitude terms to order zero only, one obtains

$$
p(\vec{r}, z, t)=\sum_{i} a_{i} \phi_{i}(z) e^{j\left(2 \pi f_{0} t-\vec{k}_{i} \vec{r}\right)}
$$

where the vector

$$
\vec{r}=(x, y),
$$

and the vector

$$
\vec{x}_{i}=\left(k_{i} \cos \beta, k_{i} \sin \theta\right)
$$

is of magnitude $k_{i}$ and points in the direction of propagation. The modal amplitude is now a function of only $r_{0}$

$$
a_{i}=\frac{S}{\rho_{0}} \sqrt{\frac{2 \pi}{k_{i} r_{0}}} \phi_{i}\left(z_{s}\right) e^{\mathcal{J}\left(\pi / 4-k_{i} r_{0}\right)}
$$

For the range dependent cases, an additional assumption of the horizontal homogeneity of the channel over the aperture of the array is needed for tractability. Given this assumption, the adiabatic theory solution (equation (2.22)) can then be converted to

$$
p(\vec{r}, z, t)=\sum_{i} a_{i} \phi_{i}\left(z, r_{0}\right) e^{j\left(2 \pi f_{0} t-\vec{\kappa}_{i} \cdot \vec{r}\right)}, .
$$


with

$$
a_{i}=b_{i}\left(r_{0}\right) e^{-j \int_{0}^{r_{0}} k_{i}(\epsilon) \epsilon d \epsilon}
$$

Here the magnitude of the wavenumber vector is the value of the horizontal wavenumber evaluated at the receiver reference point $k_{i}\left(r_{0}\right)$. Conversion of the full coupled mode solution (equation (2.20)) is more complicated, but results in the same form as for the adiabatic solution with a different definition of the modal amplitude $a_{i}$. Because the channel is assumed to have no range variations over the aperture of the array, the two sets of coupling coefficients $A_{i k}$ and $B_{i k}$ vanish in this region. A far field representation of the solution of equation (2.17) that is valid over the aperture of the array can be written as

$$
R_{i}(r)=R_{i}\left(r_{0}\right) \sqrt{\frac{r_{0}}{r}} e^{j k_{i}\left(r_{0}\right)\left(r-r_{0}\right)},
$$

where any energy scattered back to the array from channel range variations that are beyond it has been ignored. The resulting solution is then has the same form as equation (2.29), with the modal amplitude now defined as

$$
a_{i}=R_{i}\left(r_{0}\right)
$$

Again, the magnitude of the wavenumber vector is that of $k_{i}\left(r_{0}\right)$.

It can be seen that all three approaches lead to the same representation in the region of the receiver. In vector notation this may be written as

$$
p(\vec{r}, z, t)=\underline{\Psi}^{+}(\vec{r}, z) \underline{\mathbf{A}} e^{j 2 \pi \int_{0} t} .
$$

If $M$ modes are included in the model, $\underline{\mathbf{A}}$ becomes an $M \times 1$ column vector, each element of which is one of the complex modal amplitudes $\left.a_{i}, \underline{\Psi} \vec{r}, z\right)$ is also an $M \times 1$ column vector with elements 


$$
\psi_{i}(\vec{r}, z)=\phi_{i}(z) e^{j \vec{\kappa}_{i} \cdot \vec{r}}
$$

where all mode shapes and wavenumbers are those for the vertical channel structure found at the receiver. While the functional form of the modal amplitudes $a_{i}$ varies depending on the particular theory chosen, in all cases they may be considered to be constant across the aperture of the array.

The bridge from equations (2.33) and (2.34) to equations (1.4) and (1.5) is now straightforward. The total signal received on any hydrophone is assumed to consist of the signal model just developed plus additive noise. If equation (2.33) is then evaluated at each of the $N$ hydrophone locations in the receiving array, the results collected into an $N \times 1$ complex vector, and a quadrature demodulator used to remove the harmonic time dependence, equation (1.4) is recovered exactly. Each row of the $N \times M$ steering matrix is comprised of the $\underline{\Psi}^{+}$vector evaluated at a different sensor location

$$
\underline{\overline{\mathrm{E}}}=\left[\begin{array}{c}
\underline{\Psi}^{+}\left(\vec{r}_{1}, z_{1}\right) \\
\underline{\underline{\Psi}}^{+}\left(\vec{r}_{2}, z_{2}\right) \\
\dot{\Psi^{2}} \\
\dot{\Psi^{+}\left(\vec{r}_{N}, z_{N}\right)}
\end{array}\right] .
$$

Comparison with equation (1.5) allows $\theta_{i k}$ to be identified as

$$
\theta_{i k}=-\vec{\kappa}_{k} \cdot \vec{r}_{i} .
$$




\subsection{Summary}

A mathematical model to describe the sound pressure field that a harmonic point source imbedded in a waveguide generates at a distant receiving array has been developed from first principles. This model has been presented in a form to which optimal estimation theory can be applied. It has been shown that range independent mode theory, adiabatic mode theory, and coupled mode theory all lead to the same model; only the nature of the modal amplitudes is different (equations (2.28), (2.30), and (2.32), respectively). To achieve this result, both of the range dependent theories require an additional assumption of horizontal homogeneity of the channel characteristics over the aperture of the array. Subject to this assumption, the modal amplitudes can be considered constant across the array. For the range independent and adiabatic results, the modal amplitudes have been shown to be directly proportional to both the source level and the size of the mode shape at the source depth.

The long range or far field assumption has been used to justify a number of approximations. These include the neglect of either the modes in cutoff or the continuum modes, as applicable; the use of asymptoticly equivalent forms for the various range functions; and the acceptance of a local plane wave approximation in place of the actual cylindrically spreading mode.

As seen by the array, each mode appears to be a non-homogeneous plane wave propagating horizontally away from the source with unique horizontal wavenumber and depth dependence. The depth dependence of each mode is defined by its mode shape function; the various mode shapes have been shown to be orthonormal at any particular range. 


\section{Chapter 3}

\section{The FRAM IV Data Set}

The purpose of this chapter is to describe the details of the experiment during which the data set to be analyzed was taken. The nature and general layout of the experiment are first discussed. Following that are descriptions of the acoustic source and the two receiving arrays available, one a horizontally deployed twodimensional array and the other a vertical line array. Some important conclusions about practical differences between horizontal and vertical arrays are included. The details of the data acquisition and storage system and a description of the actual signals analyzed in this thesis are then presented. A discussion of the preprocessing employed prior to beamforming and an initial analysis of some of the preprocessed data complete the chapter.

\subsection{The FRAM IV Experiment}

FRAM IV, conducted in the spring of 1982 , was one of a continuing series of multi-institutional Arctic Ocean research projects sponsored by the Office of Naval Research. The experiment was designed to study the low frequency acoustical and geophysical properties of the central Arctic environment. Additionally, several physical oceanography and bottom geology experiments were conducted; besides characterizing the central Arctic region in their own right, these secondary investigations were designed to provide concurrent measurement of the environmental parameters necessary to characterize the acoustic channel from a theoretical standpoint.

The experiment was conducted from two ice camps located on the central 


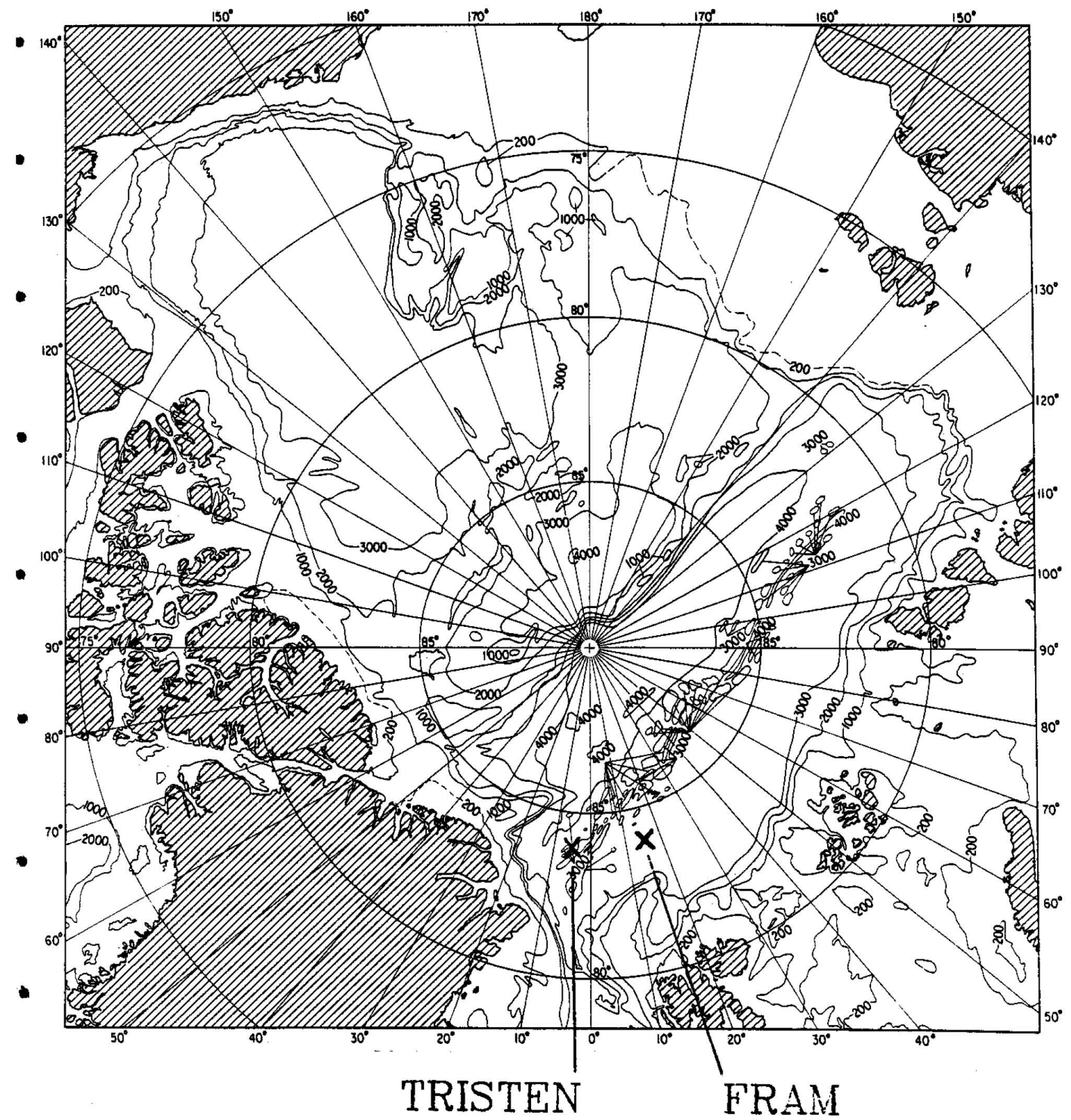

Figure 3-1: General Locations of the FRAM and TRISTEN Ice Camps 
Arctic permanent pack ice. The larger of these camps was named FRAM, and was established in the Barents Abyssal Plain, as shown in Figure 3-1. The hydrophone receiver arrays and the data acquisition systems were located here. A second camp. known as TRISTEN, was established about $300 \mathrm{~km}$ to the west of FRAM near the Mid-Arctic Ridge, primarily for the purpose of deploying a high power. low frequency acoustic source used to transmit tones and other, more complex waveforms. Explosive charges were also employed to generate impulsive source data for both acoustic and geophysical analyses. These charges were set off from various sites.

The FRAM IV data set has formed the basis for a number of recent papers; several cover topics that are germane to this thesis. The report by Tiemann, Ardai, Allen, and Manley [78] provides a full analysis of the navigation data for the experiment. Dyer [32] and Makris and Dyer [50] summarize what is currently understood about the nature and causes of Arctic ambient noise. Duckworth [29] and Duckworth and Baggeroer [30] provide detailed investigations of the bottom characteristics in the region near the FRAM camp. Mellen and DiNapoli [55] and DiNapoli and Mellen [25] characterize the propagation loss for the central Arctic and attempt to quantify the effect of the Arctic ice canopy on this propagation loss. Mikhalevsky [56], [57] investigates the temporal stability of tonal signals propagated in the Arctic channel in some detail; although the data examined in these studies were taken from the earlier FRAM II experiment, the results are equally applicable here. Polcari [67] characterizes the structure of the TRISTEN/FRAM acoustic transmission path. Finally, Yang and Giellis [84] have recently attempted modal decomposition from the FRAM IV vertical array using spatial Fourier techniques, with mixed success. 


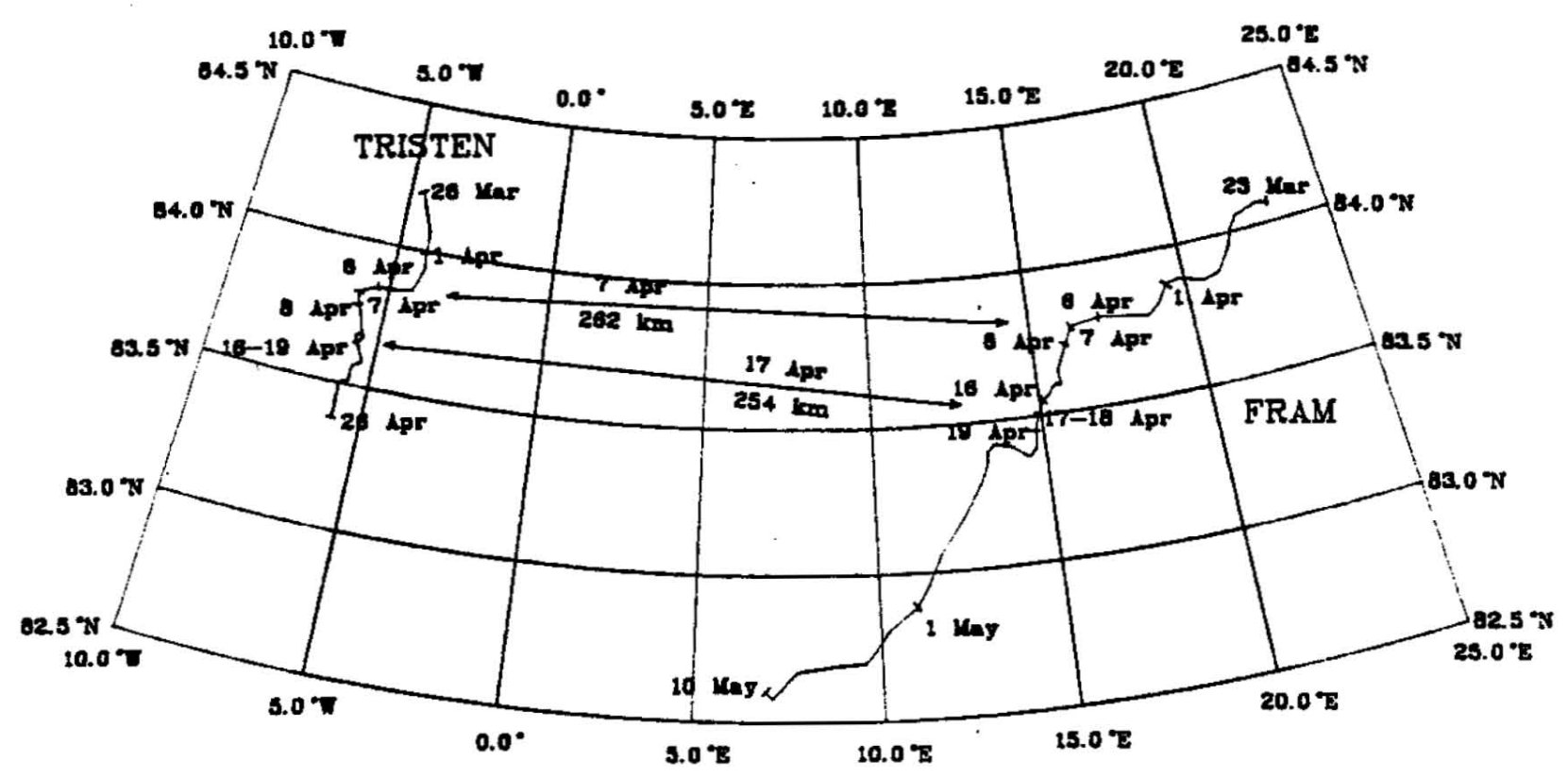

Figure 3-2: Drift of the FRAM and TRISTEN Ice Camps

\subsection{FRAM IV Navigation Data}

The FRAM receiving camp was established at $84.1^{\circ} \mathrm{N}, 23.1^{\circ} \mathrm{E}$ on $23 \mathrm{March}$, 1982 (see Figure 3-2). It drifted approximately $260 \mathrm{~km}$ to the southwest over the course of the experiment, and was abandoned at $82.6^{\circ} \mathrm{N}, 6.9^{\circ} \mathrm{E}$ on 11 May. The TRISTEN source camp was established at $84.2^{\circ} \mathrm{N}, 4.6^{\circ} \mathrm{W}$ on $26 \mathrm{March}$; it was manned until 26 April, during which time it drifted $90 \mathrm{~km}$ south to $83.4^{\circ} \mathrm{N}, 5.8^{\circ} \mathrm{W}$. The acoustic data of interest was transmitted from TRISTEN to FRAM during two separate periods of time, 6 to 8 April and 16 to 19 April. Figure 3-2 indicates representative positions for each of these periods, while Tables 3-I and 3-II tabulate the full navigation data for the same positions. All the data presented here has been derived from reference [78]. 
Date: 6 Apr 82

Time: 1800

FRAM Posit: $83.80^{\circ} \mathrm{N} \quad 16.82^{\circ} \mathrm{E}$

TRISTEN Posit: $83.84^{\circ} \mathrm{N} \quad 5.55^{\circ} \mathrm{W}$

Array Rotation: $15.6^{\circ}$ east of true North

TRISTEN Azimuth Angle: $86.5^{\circ}$

TRISTEN/FRAM Range: $265 \mathrm{~km}$

Date: 7 Apr 82

Time: 2100

FRAM Posit: $83.76^{\circ} \mathrm{N} \quad 16.08^{\circ} \mathrm{E}$

TRISTEN Posit: $83.81^{\circ} \mathrm{N} \quad 5.88^{\circ} \mathrm{W}$

Array Rotation: $15.9^{\circ}$ east of true North

TRISTEN Azimuth Angle: $86.1^{\circ}$

TRISTEN/FRAM Range: $262 \mathrm{~km}$

Date: 8 Apr 82

Time: 1530

FRAM Posit: $83.72^{\circ} \mathrm{N} \quad 15.96^{\circ} \mathrm{E}$

TRISTEN Posit: $83.78^{\circ} \mathrm{N} \quad 5.83^{\circ} \mathrm{W}$

Array Rotation: $16.2^{\circ}$ east of true North

TRISTEN Azimuth Angle: $86.1^{\circ}$

TRISTEN/FRAM Range: $262 \mathrm{~km}$

Table 3-I: TRISTEN/FRAM Navigation Data for the Horizontal Array Data Set

\subsection{Horizontal Azimuth Angle Conventions}

Before continuing, a short comment on the measurement conventions used in this work for horizontal direction angles is in order. Whenever incoming signal horizontal azimuth angle values are quoted, the convention implied throughout the thesis is that the azimuth angle has been measured from the reference direction to the direction in which the signal is propagating in a clockwise direction. This makes the azimuth angle exactly the reciprocal angle of the more traditional bearing angle. As a simple example, a signal propagating from a source to the northwest arrives on a bearing of $315^{\circ}$; since the signal itself is traveling towards 
Date: 16 Apr 82

Time: 1430

FRAM Posit: $83.55^{\circ} \mathrm{N} \quad 15.04^{\circ} \mathrm{E}$

TRISTEN Posit: $83.66^{\circ} \mathrm{N} \quad 5.70^{\circ} \mathrm{W}$

Array Rotation: $17.6^{\circ}$ east of true North

TRISTEN Azimuth Angle: $85.6^{\circ}$

TRISTEN/FRAM Range: $255 \mathrm{~km}$

Date: 17 Apr 82

Time: 1930

FRAM Posit: $83.49^{\circ} \mathrm{N} \quad 14.86^{\circ} \mathrm{E}$ TRISTEN Posit: $83.66^{\circ} \mathrm{N} \quad 5.69^{\circ} \mathrm{W}$

Array Rotation: $17.4^{\circ}$ east of true North

TRISTEN Azimuth Angle: $86.8^{\circ}$

TRISTEN/FRAM Range: $254 \mathrm{~km}$

Date: 19 Apr 82

Time: 0300

FRAM Posit: $83.48^{\circ} \mathrm{N} \quad 14.77^{\circ} \mathrm{E}$

TRISTEN Posit: $83.64^{\circ} \mathrm{N} \quad 5.59^{\circ} \mathrm{W}$

Array Rotation: $17.1^{\circ}$ east of true North

TRISTEN Azimuth Angle: $87.2^{\circ}$

mEN/FRAM Range: $253 \mathrm{~km}$

fnr the Vertical Array Data Set 
significant, especially when high resolution beamforming techniques are employed. Tables 3-I and 3-II both include appropriate array rotation data.

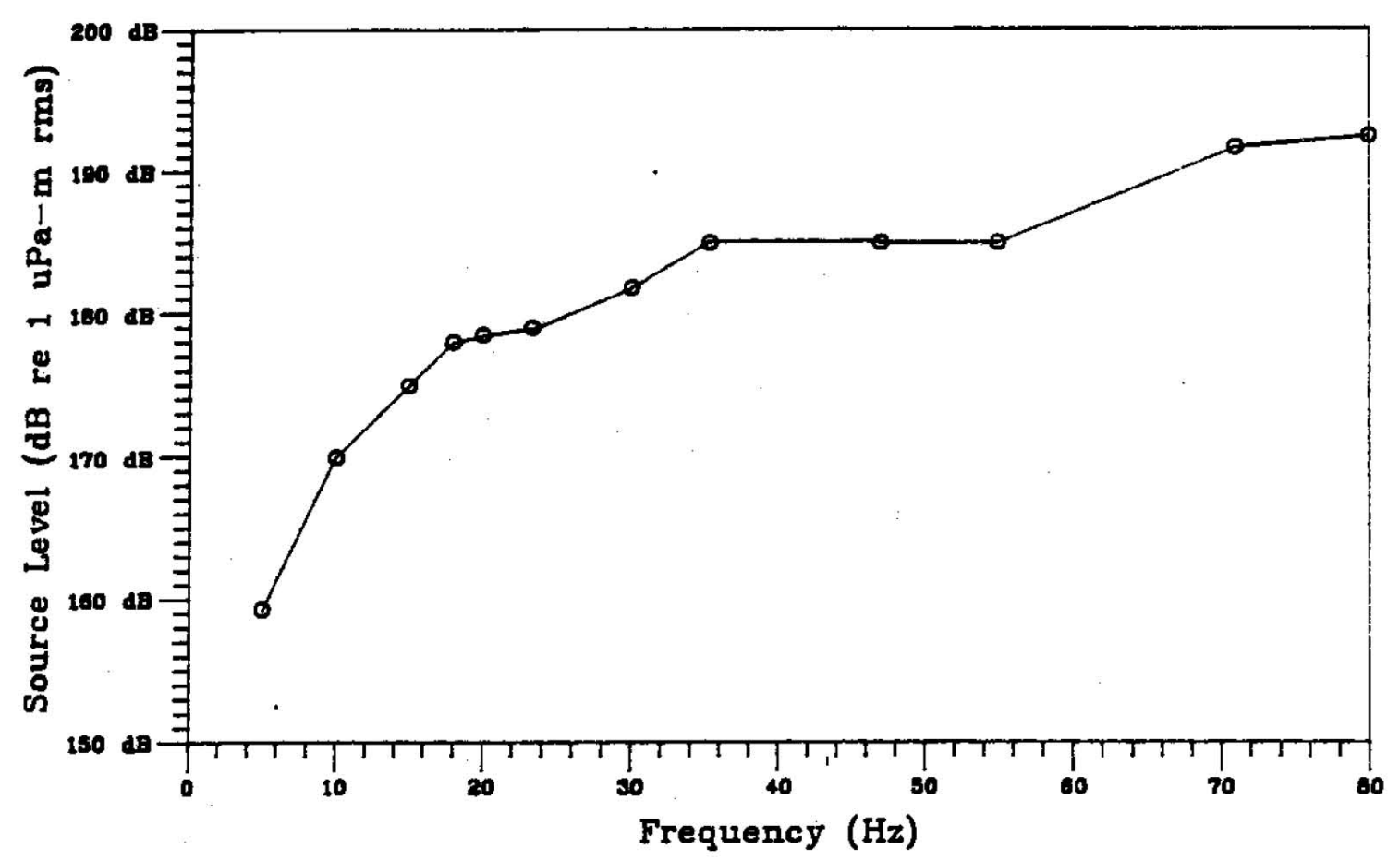

Figure 3-3: Source Levels for the TRISTEN Source

\subsection{The TRISTEN Low Frequency Source}

A modified HLF low frequency source was deployed to a depth of $91 \mathrm{~m}$ at the TRISTEN camp. Approximately 150 hours of signals were broadcast in a frequency range from 5 to $110 \mathrm{~Hz}$. Several different waveform types are included in the signal set. Of interest here are a series of tonal signals in the range from 5 to $71 \mathrm{~Hz}$. The typical signal format consists of one tone per hour, with the source on continuously for the first 55 minutes and then off for the last 5 minutes of the hour. Scheduling constraints often forced more abbreviated formats; most of the horizontal array 
data used here was broadcast at half-hour intervals rather in the full hour format, for example. The source has a rated frequency stability of better than one part in $10^{6}$; it can therefore be considered to be temporally coherent over the full duration of any signal investigated in this thesis. Figure 3-3 provides a plot of source levels versus frequency in the range from 5 to $80 \mathrm{~Hz}$ for the TRISTEN source. This data was extracted from reference [26].
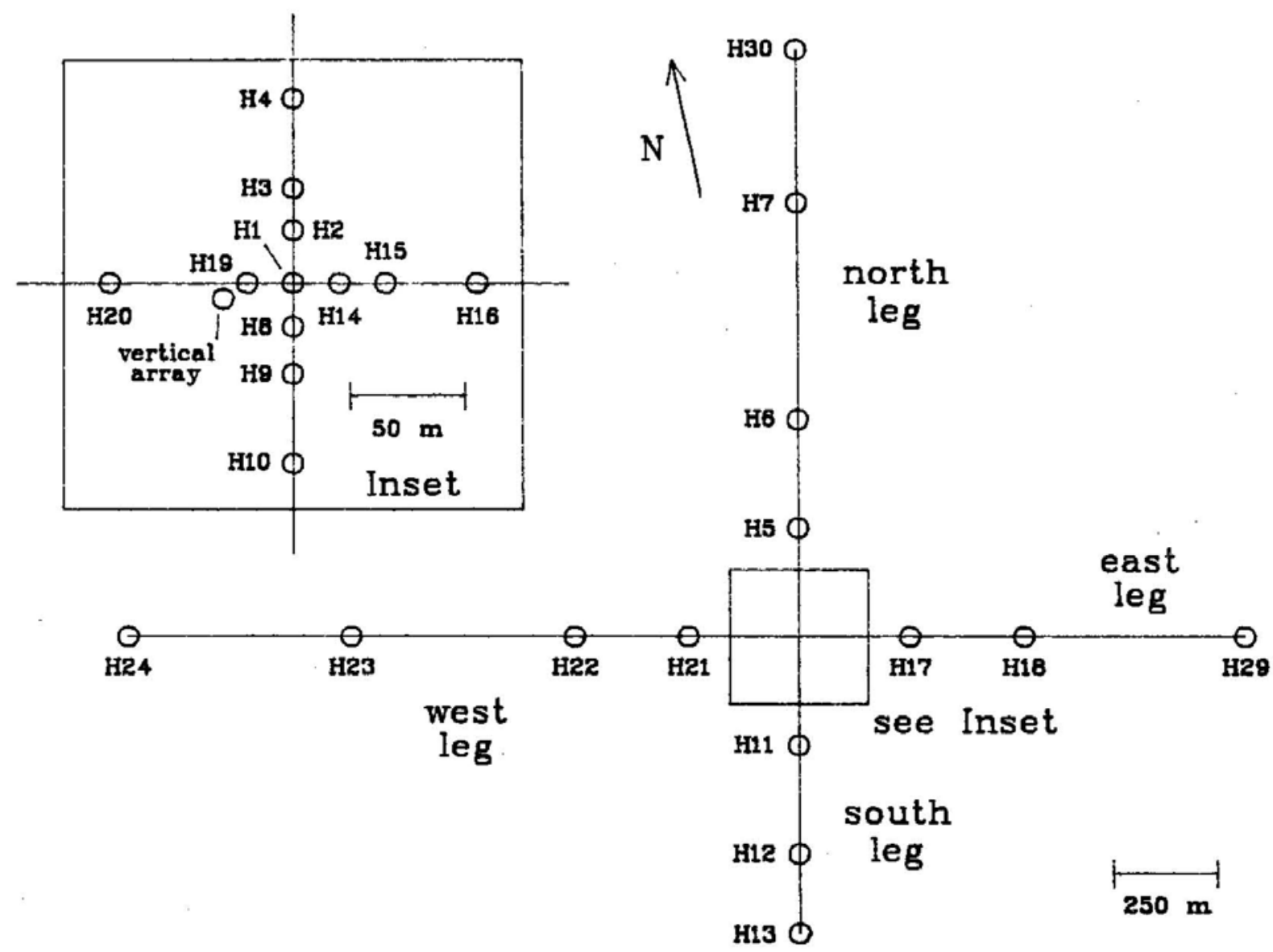

Figure 3-4: Arrangement of the FRAM Receiving Arrays 


\subsection{The FRAM IV Receiving Arrays}

Two receiving arrays were available at the FRAM camp. A two-dimensional horizontal array consisting of 26 elements was deployed through the ice and suspended on cables to a depth of $91 \mathrm{~m}$. The net gain to the recording system was $-159 \mathrm{~dB}$ re $1 \mathrm{v} / \mu \mathrm{Pa}$, with a flat frequency response to below $2 \mathrm{~Hz}$. As shown in Figure 3-4, the array was deployed in an cross-shaped configuration. Each leg of the array had an aperture of about $1 \mathrm{~km}$, with individual elements spaced approximately logarithmically from the center.

The second array was made up of 28 elements and extended vertically into the water column to a depth of approximately $1000 \mathrm{~m}$. Since the bottom depth at the receiver camp averaged $3800 \mathrm{~m}$, this coverage represents the top $25 \%$ of the channel. The elements in the array were spaced approximately linearly. The array was located about $30 \mathrm{~m}$ to the southwest of the center of the horizontal array, as is also indicated on Figure 3-4. The net gain from the water to the recording system was $-121 \mathrm{~dB}$ re $1 \mathrm{v} / \mu \mathrm{Pa}$, with the exception of a few particular hydrophones where the gain was reduced to $-127 \mathrm{~dB}$ in order to reduce amplifier saturation. The frequency response was flat above $6 \mathrm{~Hz}$. A $500 \mathrm{lb}$ weight was hung from the bottom of the array to stabilize it against current action.

Both arrays suffered to varying degrees from two related problems endemic to suspended sensor systems, sensor displacement and strum. Sensor displacement is the offset of a particular hydrophone from its nominal position caused by current drag on the hydrophone and its support cable. Since only the position at which the sensor is deployed though the ice is known, such displacements translate into phase errors in the beamforming process. Simple delay-and-sum beamforming theory indicates that sensor displacements become significant when they begin to reach 
magnitudes of approximately one quarter wavelength; the problem obviously gets more severe with increasing frequency or more sophisticated beamforming algorithms. Modal decompositions may be even more affected than other techniques when, as for the vertical array, the errors occur across the array in a consistent fashion, causing the array to become tilted. This sensitivity is investigated in detail in Chapter 6. Time varying sensor displacements are possible due both to variations in the environmental conditions and possible pendulum oscillations of the sensors. As discussed below and in Chapter 7, however, the time variations of sensor displacements observed in the data appear to be relatively small when compared to the static offsets.

Strum is the vibration of the suspension cable of a sensor due to vortices shed by the surrounding current flow. This vibration causes slight oscillations in the sensor depth; since the ambient pressure is a function of depth, these depth excursions cause pressure field variations to which the sensor then responds. As opposed to sensor displacement, which occurs on time scales so large that its primary effect is a phase modulation of the received signal, strum energy is concentrated at the lowest frequencies of the acoustic domain, and forms an additive noise source from which the true signal must then be separated. Strumming action generally appears to consist of a fundamental frequency and a number of strong harmonics. These harmonics typically occupy the frequency range below $10 \mathrm{~Hz}$ for horizontal array sensors; significant harmonics can be found at frequencies as high as $30 \mathrm{~Hz}$ during severe episodes, however. Because different horizontal hydrophones exhibit varying strum patterns, spatial processing discriminates well against strum energy. The greater effective diameter of its support cable causes strum effects for the vertical array to occupy a lower frecquency range; strum energy above $10 \mathrm{~Hz}$ is rarely observed in vertical array data, making it 
much less of a problem than for the horizontal array. These observations agree with strum frequency predictions based on the Strouhal number, which yield expected strum frequencies of $28.5 \mathrm{~Hz}$ for the horizontal array and $5.1 \mathrm{~Hz}$ for the

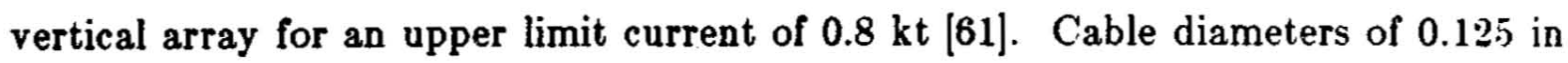
for the horizontal array elements and 0.7 in for the vertical array are assumed in these calculations.

\subsection{The Effects of Hydrophone Sensitivity Mismatches}

An important difference between the horizontal and vertical arrays involves the effect of variations in hydrophone sensitivity and amplification gain across the arrays. For a horizontal array of reasonable aperture, the signal received at each sensor is a time shifted replica of that found at other hydrophones, as is demonstrated in Figure 1-1. This assumption is often used in horizontal array beamforming to eliminate variations in the receiver hardware by artificial normalization of each time series before beamforming. This can be done because a horizontal array uses the signal phase rather than its amplitude to develop spatial information.

Since a vertical array samples the modal structure of the channel, however. signal amplitude variations between different hydrophones are both expected and desired; a normalization scheme as might be used on a horizontal array is no longer applicable. Thus, the nature of the data and the processing make hardware uniformity between channels a much more important issue for vertical arrays than for horizontal arrays. No indication of this type of problem is seen anywhere in the FRAM IV data set; none the less, the issue must be carefully considered whenever one is dealing with data taken from vertical arrays. 


\subsection{Sensor Displacement Predictions for the Horizontal and Vertical Arrays}

In general, the effect of sensor displacement on the horizontal array is significantly less severe than its effect on the vertical array. This is to be expected, because the vertical array extends approximately ten times deeper into the water column than the horizontal array sensors; the longer scope then makes it more sensitive to current effects. The purpose of this section is to investigate the significance of the sensor displacements likely to be encountered in practice for both horizontal and vertical array sensors. As the purpose is not to make precise quantitative predictions, but rather to provide some feel for when sensor displacements must be considered and when they might be safely ignored, some very simple hydrodynamic approximations suffice.

The actual sensor displacement is the primary parameter of interest for a horizontal array sensor. For the vertical array, however, things are somewhat more complicated, as many sensors are suspended from the same cable. Obviously. the maximum sensor displacement achieved is important; this displacement will generally be found at the deepest sensor in the array, although more complicated geometries are possible. Additionally, however, the distribution of displacement along the vertical array is significant. One simple way of parameterizing this distribution is by some type of tilt angle, representing the slope of some notional line with which one has replaced the actual array shape. Several different tilt angle definitions are possible. The one used here is simply the angle made with the vertical by a line drawn through the array anchor point at the surface and the bottom sensor. A more rigorous definition is introduced in Chapter 6, where the justification for such an approximation is discussed. A similar parameter of interest for both horizontal and vertical arrays is the slope of the suspension cable at the 
surface, since it provides a check against field experience.

The sensor displacement problem may be modeled in a very simple fashion by assuming the sensor suspension cable to be a uniform line of length $H$ with one end fixed to the ice at the origin and the other end attached to a concentrated mass of weight $W$ that represents the weight normally deployed at the end of the cable for current stabilization. This model is applicable for both the horizontal and vertical arrays, with one difference: for the vertical array, the mass of the line must be considered, whereas for the horizontal array it is negligible. Let $w$ be this weight per unit length of the cable. To keep the hydrodynamies simple, the line is assumed to have a uniform diameter $d$, and the current $V$ is assumed to be constant in depth. The first assumption is not strictly correct for the vertical array, since the actual arrangement of the support cable and the electrical connections from the hydrophones to the surface is rather complicated; this is compensated for by using some type of effective diameter over the length of the cable. Similarly, basic oceanography indicates that the second assumption is not very realistic either. However, both of these assumptions will more often cause the displacement estimates at the deepest sensors to be too large rather than too small. A secondary issue involves the three-dimensional nature of the array shape, which is not reflected by the constant current assumption. This problem is not serious, however, because in the sequel it is not the actual array shape that is important, but only its projection in the vertical plane of propagation.

Assuming a steady state situation, the primary effect of the current is to provide a constant horizontal drag force per unit length $f$ on the line and a total drag force $F$ on the concentrated mass suspended from it. The drag force per unit. length of line is estimated from two-dimensional Reynolds number theory [61] to be 


$$
f=\frac{C_{D} \rho V^{2} d}{2}
$$

where $\rho$ is the density of water and $C_{D}$ is a two-dimensional drag coefficient that is a function of the Reynolds number

$$
R=\frac{V d}{\nu}
$$

Here, $\nu$ is the kinematic viscosity of water. A similar but somewhat less reliable estimate may be made for $F$.

Given estimates for the two drag forces, one can then solve the resulting statics problem for the shape of the line as a function of depth in a straightforward manner. The solution is

$$
r(z)=a[z-b \ln (1-z / c)]
$$

where

$$
\begin{aligned}
& a=\frac{f}{w}, \\
& b=\frac{F}{f}-\frac{W}{w},
\end{aligned}
$$

and

$$
c=\frac{W}{w}+H
$$

For the vertical array, $H$ is assumed to be $960 \mathrm{~m}$, and $w$ and $W$ may be taken to be $17.8 \mathrm{~N} / \mathrm{m}(4 \mathrm{lb} / \mathrm{m})$ and $2224 \mathrm{~N}(500 \mathrm{lb})$, respectively. Using an effective cable diameter of 0.7 in and a current of $0.8 \mathrm{kt}$ (a reasonable upper limit for the speed of ice movement observed in the Arctic), the $\operatorname{drag}$ forces $f$ and $F$ are estimated to be $1.5 \mathrm{~N} / \mathrm{m}$ and $10.5 \mathrm{~N}$, respectively. These numbers give values of 0.087 for $a,-118 \mathrm{~m}$ for $b$, and $1085 \mathrm{~m}$ for $c$. 

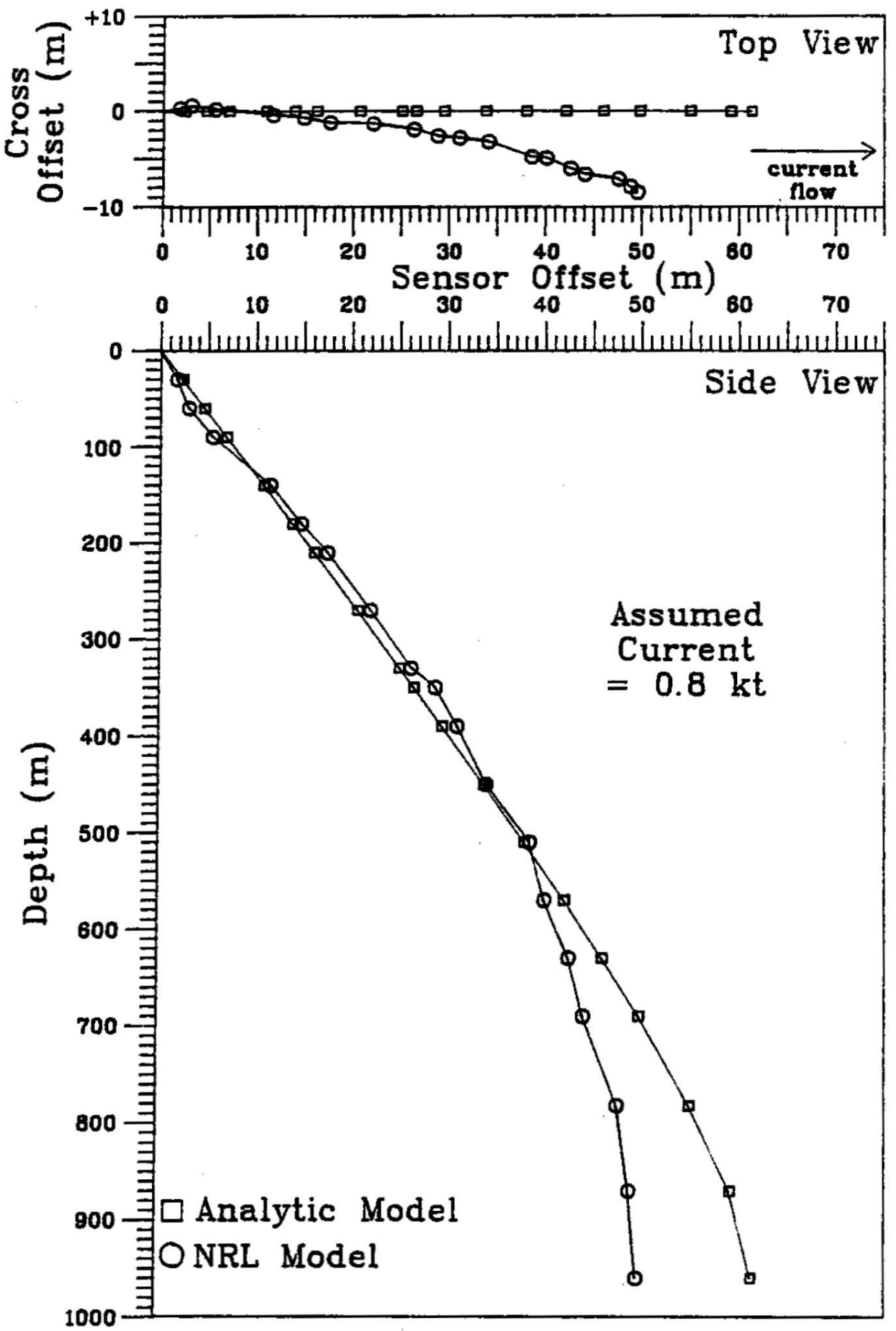

Figure 3-5: Two Models for the FRAM Vertical Array Shape (second model taken from [85])

[Note different depth and offset scales.] 
Equation (3.3) is plotted as a function of depth in Figure 3-5 for the values just discussed. The results of a more sophisticated model developed at the Naval Research Laboratory (NRL) [85] are also shown for comparison. The two models agree quite well in the first $600 \mathrm{~m}$, and then diverge slowly after that. A maximum difference of about $10 \mathrm{~m}$ between the two predictions occurs at the bottom of the array. Several different factors probably contribute to the difference between the two models, but the most important is the assumption of a current that is constant with depth made in the simpler model.

As shown in Figure 3-5, the sensor displacements indicated for the lowest hydrophones are in the 50 to $60 \mathrm{~m}$ range, making them a significant fraction of a wavelength at even the lowest frequencies of interest. The array tilt as measured from the anchor point at the surface to the bottom sensor is $3.6^{\circ}$; the slope of the array at the surface is $5.0^{\circ}$, which agrees well with field experience, in which slopes of no more than about 5 or $6^{\circ}$ have been observed. The equivalent values for the NRL model are $3.0^{\circ}$ and $3.8^{\circ}$, respectively. These results are in general agreement with other field tests carried out by NRL in the Arctic for the same array [69]. It is important to note that the magnitude of the tilt angle is fundamentally proportional to the square of the current $V$.

The same approach outlined above can be used for the horizontal array sensors. In this case the weight of the cable $w$ is negligible, and the resultant shape of the line takes on a somewhat simpler parabolic form

$$
r(z)=\frac{z}{W}[F+f(H-z / 2)]
$$

For a horizontal array hydrophone, $H$ is $91 \mathrm{~m}$ and $W$ may be taken to be $133.4 \mathrm{~N}$ (30 lb). For a cable diameter of 0.125 in and a current of $0.8 \mathrm{kt}$, the drag forces $f$ and $F$ are computed to be $0.277 \mathrm{~N} / \mathrm{m}$ and $1.688 \mathrm{~N}$, respectively. These numbers 
yield an offset of about $10 \mathrm{~m}$, which corresponds to a quarter wavelength at roughly $35 \mathrm{~Hz}$, and a cable slope at the surface of $11.4^{\circ}$. These results agree with with operational experience, which indicates that beamforming degradations are first. noticable in the 30 to $40 \mathrm{~Hz}$ frequency band.

Several conclusions can be drawn from this section. The most important is that sensor displacements for the horizontal array can be expected to be negligible below about $30 \mathrm{~Hz}$, while those of the vertical array can never be totally ignored. Additionally, typical offsets for the vertical array can be expected to be about five times those of the horizontal array, primarily due to the much longer array scope. Finally, the angle that the vertical array makes with the true vertical can be expected to be limited to a range of about $5^{\circ}$ based on the range of currents likely to be encountered in the central Arctic. All of these observations are of importance in Chapter 6, where the problem of vertical array tilt is examined in detail.

\subsection{The FRAM IV Data Acquisition System}

The recording system is illustrated in Figure 3-6. It consisted of a minicomputer-based digital data acquisition and storage system. Twenty-four channels of information, each sampled at $250 \mathrm{~Hz}$, were digitized simultaneously; the resulting digital data were then stored on specially formatted 1600 BPI magnetic tapes. An analog filter with a roll off of $48 \mathrm{~dB} /$ octave above the corner frequency of $80 \mathrm{~Hz}$ prevented aliasing, and a floating point conversion scheme preserved the required dynamic range. The well matched phase responses of the various channels preserved the signal phase synchronization required for beamforming. The input to each of the 24 channels was selectable via a patch panel. Several different sensors sets were recorded during the course of the experiment. Further technical details of the recording system can be found in Prada, et al. [71]. 


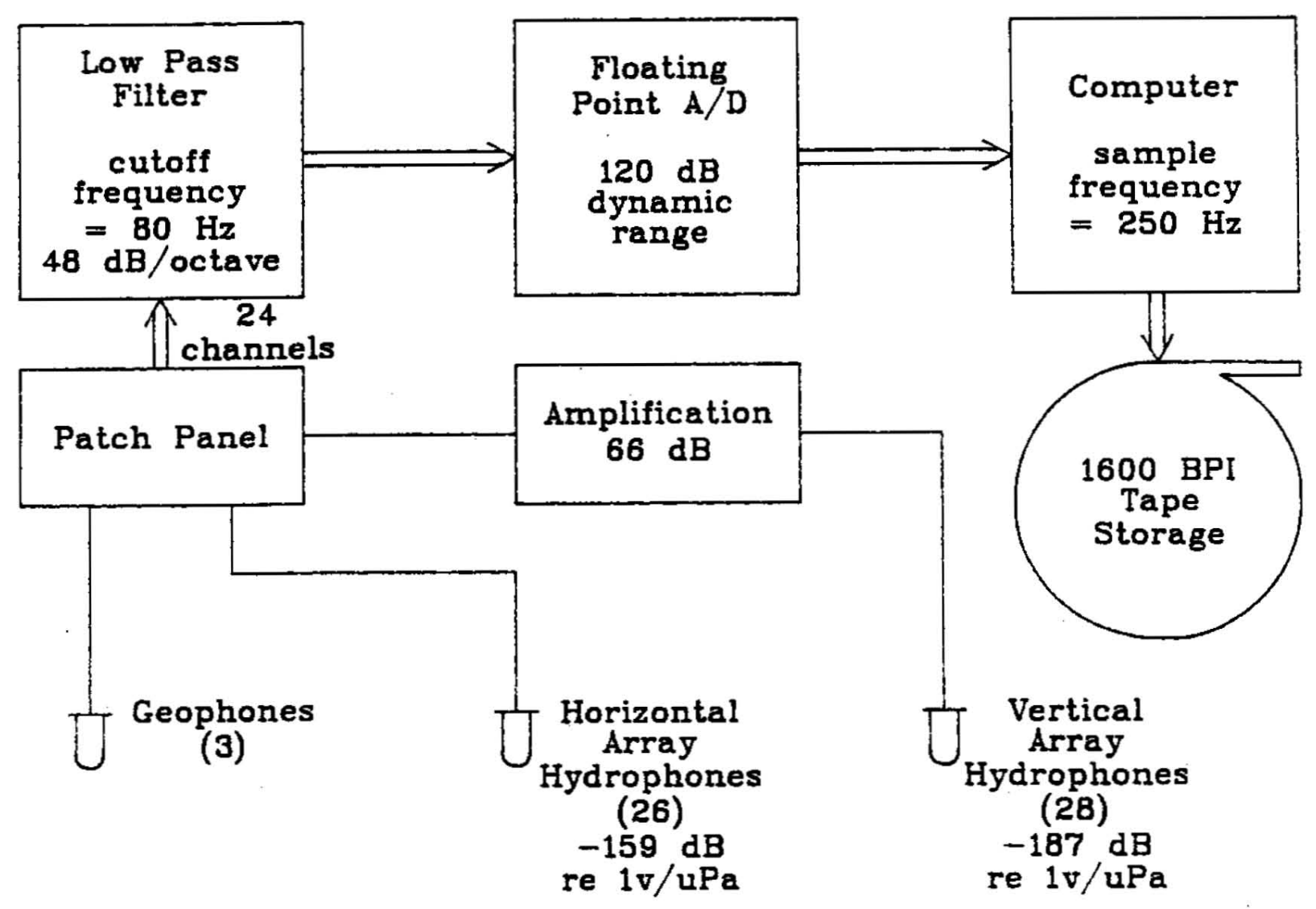

Figure 3-6: The FRAM IV Digital Recording System

The FRAM IV acoustic data is classified according to the array configuration (i.e., the sensor set) being recorded when the data was acquired. Two major types of array configurations exist. The data taken from 6 to 8 April were recorded from only horizontal array hydrophones. Over this period minor variations were made in the exact hydrophone set recorded, resulting in slightly different array configurations for different signals. These variations are not significant when considered in terms of theoretical array performance, however. The data taken from 16 to 19 April was recorded from a mixture of horizontal and vertical array hydrophones. The details of the sensor set chosen for this configuration are 
provided in Table 3-III. Of the most interest here are the last eighteen channels, which were used to record selected hydrophones of the vertical array; the depths of the sensors recorded are included in Table 3-III. In processing the data taken from this vertical array configuration, only these channels have been used, so that an eighteen element fully vertical array has been effectively synthesized. This has been done to allow assessment of the operational utility of a true vertical array.

\begin{tabular}{c} 
Channel \\
\hline 1 \\
2 \\
3 \\
4 \\
5 \\
6 \\
7 \\
8 \\
9 \\
10 \\
11 \\
12 \\
13 \\
14 \\
15 \\
16 \\
17 \\
18 \\
$19 *$ \\
$20^{*}$ \\
21 \\
22 \\
23 \\
24 \\
\end{tabular}

\author{
Description \\ Horizontal array center phone \\ Horizontal array northernmost phone \\ Horizontal array southernmost phone \\ Horizontal array easternmost phone \\ Horizontal array westernmost phone \\ Geophone \\ Vertical array phone at $960 \mathrm{~m}$ \\ Vertical array phone at $860 \mathrm{~m}$ \\ Vertical array phone at $782 \mathrm{~m}$ \\ Vertical array phone at $690 \mathrm{~m}$ \\ Vertical array phone at $6.30 \mathrm{~m}$ \\ Vertical array phone at $570 \mathrm{~m}$ \\ Vertical array phone at $510 \mathrm{~m}$ \\ Vertical array phone at $450 \mathrm{~m}$ \\ Vertical array phone at $390 \mathrm{~m}$ \\ Vertical array phone at $350 \mathrm{~m}$ \\ Vertical array phone at $330 \mathrm{~m}$ \\ Vertical array phone at $270 \mathrm{~m}$ \\ Vertical array phone at $210 \mathrm{~m}$ \\ Vertical array phone at $180 \mathrm{~m}$ \\ Vertical array phone at $140 \mathrm{~m}$ \\ Vertical array phone at $90 \mathrm{~m}$ \\ Vertical array phone at $60 \mathrm{~m}$ \\ Vertical array phone at $30 \mathrm{~m}$
}

*These hydrophones had net gain to recording system of

$-127 \mathrm{~dB}$ rather than the nominal $-121 \mathrm{~dB}$ (re $1 \mu \mathrm{Pa} / \mathrm{v}$ ).

Table 3-III: The FRAM IV Vertical Array Recording Configuration 


\subsection{Details of the Vertical Array Data Set}

The objectives of this thesis make the data recorded from the vertical array of primary interest. The available data set consists of tonals at fourteen different frequencies between 5 and $71 \mathrm{~Hz}$ recorded from 16 to 19 April. These are listed in Table 3-IV. Many of the frequencies were broadcast several times over the course of the four days; only the longest representative data segment at each frequency has been chosen for analysis here. The three lowest frequencies were never detected in either the vertical array data set or the more extensive horizontal array data set. This is most likely due to insufficient signal to noise ratios at these frequencies. Not only does the source exhibit significantly lower signal levels below $15 \mathrm{~Hz}$ (as shown in Figure 3-3), but, in addition, Arctic ambient noise tends to increase with decreasing frequency in this band [32].

\begin{tabular}{|c|c|c|c|}
\hline Frequency & Time & Date & Duration \\
\hline $5.00 \mathrm{~Hz}$ & & Not Detected & \\
\hline $10.00 \mathrm{~Hz}$ & & Not Detected & \\
\hline $11.75 \mathrm{~Hz}$ & & Not Detected & \\
\hline $15.00 \mathrm{~Hz}$ & 0310 & 19 Apr 82 & $10 \mathrm{~min}$ \\
\hline $17.75 \mathrm{~Hz}$ & 1430 & 16 Apr 82 & $55 \mathrm{~min}$ \\
\hline $20.00 \mathrm{~Hz}$ & 0225 & 19 Apr 82 & $35 \mathrm{~min}$ \\
\hline $23.50 \mathrm{~Hz}$ & 0330 & 18 Apr 82 & $5.5 \mathrm{~min}$ \\
\hline $30.00 \mathrm{~Hz}$ & 0320 & 19 Apr 82 & $10 \mathrm{~min}$ \\
\hline $35.25 \mathrm{~Hz}$ & 0430 & 18 Apr 82 & $55 \mathrm{~min}$ \\
\hline $35.50 \mathrm{~Hz}$ & 1030 & 17 Apr 82 & $55 \mathrm{~min}$ \\
\hline $47.00 \mathrm{~Hz}$ & 1830 & 17 Apr 82 & $55 \mathrm{~min}$ \\
\hline $53.25 \mathrm{~Hz}$ & 0930 & 17 Apr 82 & $55 \mathrm{~min}$ \\
\hline $55.00 \mathrm{~Hz}$ & 1130 & 17 Apr 82 & $55 \mathrm{~min}$ \\
\hline $71.00 \mathrm{~Hz}$ & 1530 & 17 Apr 82 & $55 \mathrm{~min}$ \\
\hline
\end{tabular}

Table 3-IV: The FRAM IV Vertical Array Data Set 


\subsection{Details of the Horizontal Array Data Set}

To confirm the results generated from the vertical array data set, a smaller number of tonal signals selected from the more extensive horizontal array data set have also been analyzed. The particular segments chosen are listed in Table 3-V. In general, the frequencies taken from the horizontal array have been chosen to closely match those available in the vertical array data set. Several of the segments are rather short, making them inappropriate for use in investigating long term signal stability issues; none the less, they are still valuable for comparing received signal levels with those of the vertical array.

\begin{tabular}{llcc} 
Frequency & Time & Date & $\frac{\text { Duration }}{30 \mathrm{~min}}$ \\
\hline $15.00 \mathrm{~Hz}$ & 2330 & $7 \mathrm{Apr} 82$ & $30 \mathrm{~min}$ \\
$17.75 \mathrm{~Hz}$ & 1800 & $7 \mathrm{Apr} 82$ & $30 \mathrm{~min}$ \\
$23.50 \mathrm{~Hz}$ & 1830 & $7 \mathrm{Apr} 82$ & $30 \mathrm{~min}$ \\
$27.00 \mathrm{~Hz}$ & 1530 & $8 \mathrm{Apr} 82$ & $30 \mathrm{~min}$ \\
$35.25 \mathrm{~Hz}$ & 1930 & $7 \mathrm{Apr} 82$ & $13 \mathrm{~min}$ \\
$47.00 \mathrm{~Hz}$ & 2030 & $7 \mathrm{Apr} 82$ & $8 \mathrm{~min}$ \\
$53.25 \mathrm{~Hz}$ & 2000 & $7 \mathrm{Apr} 82$ & $30 \mathrm{~min}$
\end{tabular}

Table 3-V: The FRAM IV Horizontal Array Data Set

\subsection{Preprocessing of the Data}

The extensive nature of the data set (the signal set exceeds 1500 Mbytes of data as recorded in the field) makes analysis of the data in a raw form impractical. The preprocessing scheme shown in Figure 3-7 has therefore been developed to filter and compress the data set to a tractable size. The field data for each sensor (sampled at $250 \mathrm{~Hz}$ ) is initially demodulated and low pass filtered to produce a complex time series of the signal amplitude and phase. For reasons of efficiency and phase stability, the demodulation is implemented in the frequency domain, through 
First Stage

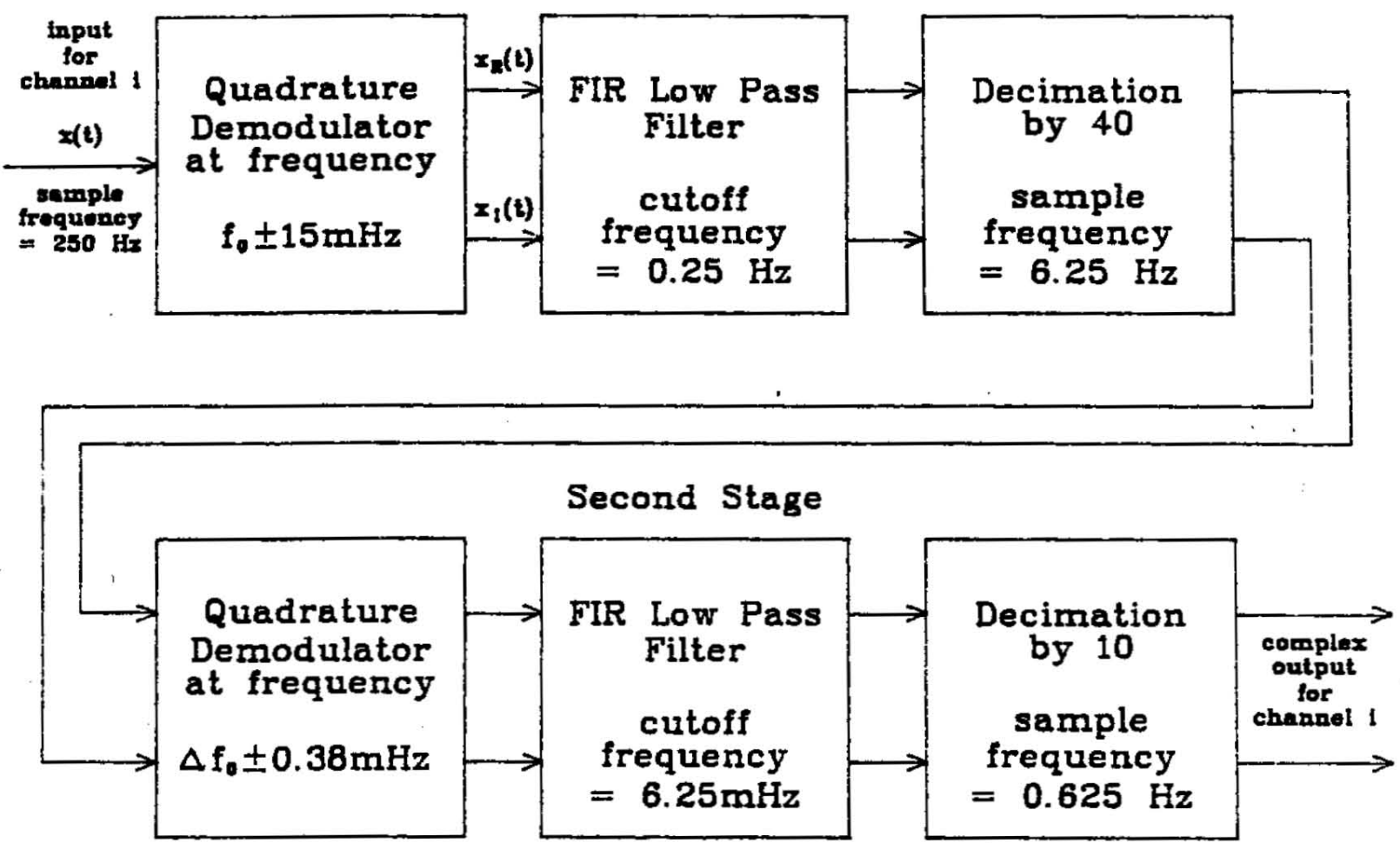

Figure 3-7: The Preprocessing Scheme

the use of bin shifts in the FFT of the data. This approach has the disadvantage that nominal signal frequency can not be achieved exactly; the 8192 point length of the FFT used provides resolution only to within $\pm 15 \mathrm{mHz}$ of the desired frequency. The need to generate quadrature sinusoids with adequate phase stability over the signal durations involved is avoided, however; a typical signal of one hour in length spans about $10^{5}$ cycles. With the frequency domain implementation, this phase stability is controlled only by the frequency stability of the highly accurate sample clock. The scheme also increases computational efficiency by allowing the simultaneous implementation of a Parks-McClellan FIR low pass filter [63] in the frequency domain. The amplitude and phase responses of this 399 point filter are 


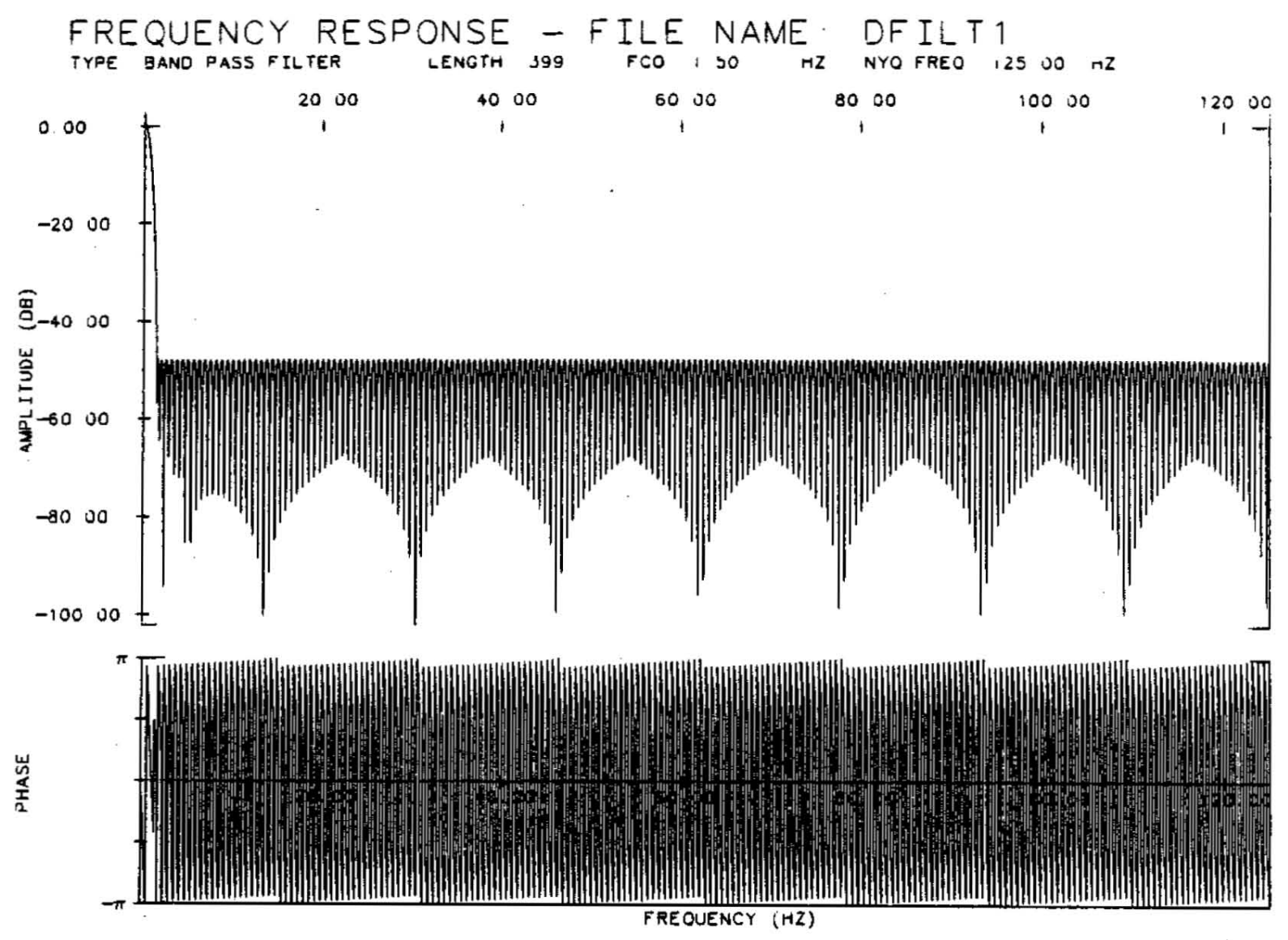

Figure 3-8: Parks-McClellan FIR Filter Frequency Response

presented in Figure 3-8, and its characteristics are provided in Table 3-VT. The filter is designed to provide as narrow a passband as possible while retaining adequate cutoff levels in the rejection band. The filter length is limited by the particular Parks-McClellan algorithm available, which can handle no larger than 400 point filters. After complex demodulation and low pass filtering, the dat a is then decimated by a factor of 40 (to a $6.25 \mathrm{~Hz}$ sample rate); the FIR filter characteristics guarantee that the Nyquist criterion is met.

Following this first stage of preprocessing, an identical second stage of complex demodulation, low pass filtering, and decimation is accomplished. The 
second stage processing provides further refinement of the demodulation freculuency and a tighter low pass filter, allowing reductions in the number of data points to convenient levels. Because of the reduced sampling period at the input to the second stage, a demodulation frequency resolution of better than $1 \mathrm{mHz}$ can now be achieved. The FIR filter of Figure $3-8$ is again applied, this time resulting in a passband of $12.5 \mathrm{mHz}$ (compared to the $500 \mathrm{mHz}$ bandwidth available in the first stage). Finally, the data is once more decimated, this time by a factor of 10 .

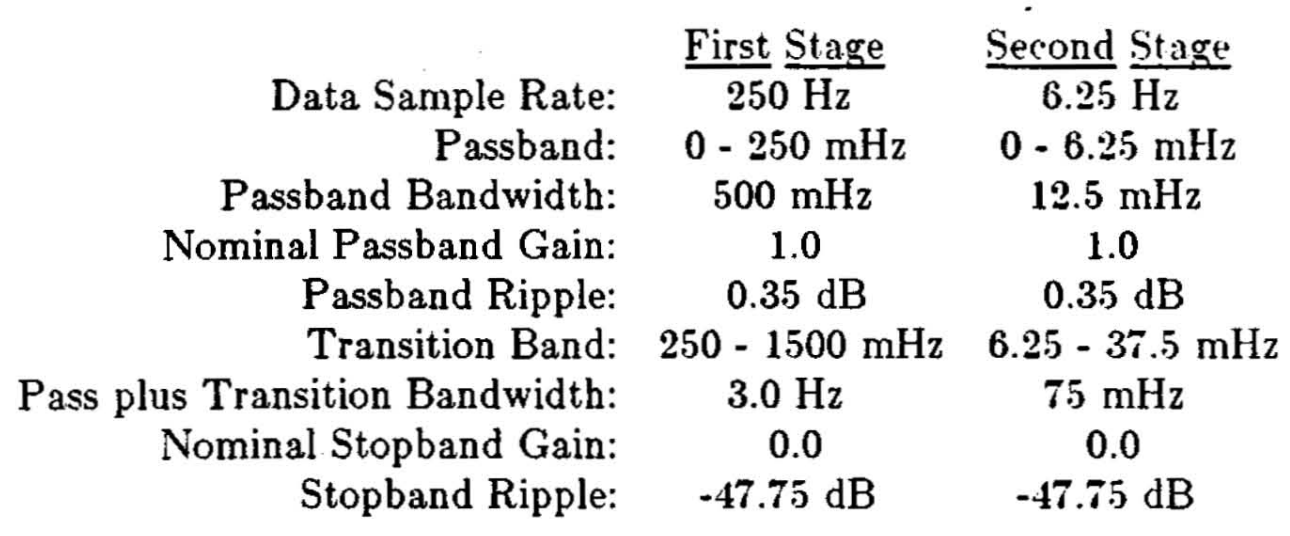

Table 3-VI: FIR Filter Characteristics

Both stages of the preprocessing described above have been carried out on all 24 channels of data simultaneously, resulting in preprocessed complex time series that are demodulated to within $1 \mathrm{mHz}$ of the nominal signal frequency and then very closely filtered around that frequency. The final sample frequency is $0.625 \mathrm{~Hz}$ (equivalent to a sample period of $1.6 \mathrm{sec}$ ), providing approximately 2000 data points per channel for a signal of 55 minute duration. As the correlation length of the second stage low pass filter is about 20 post-decimated points, these 2000 points represent roughly 100 independent degrees of freedom. Further details of the digital signal processing involved can be found in reference [68]. 


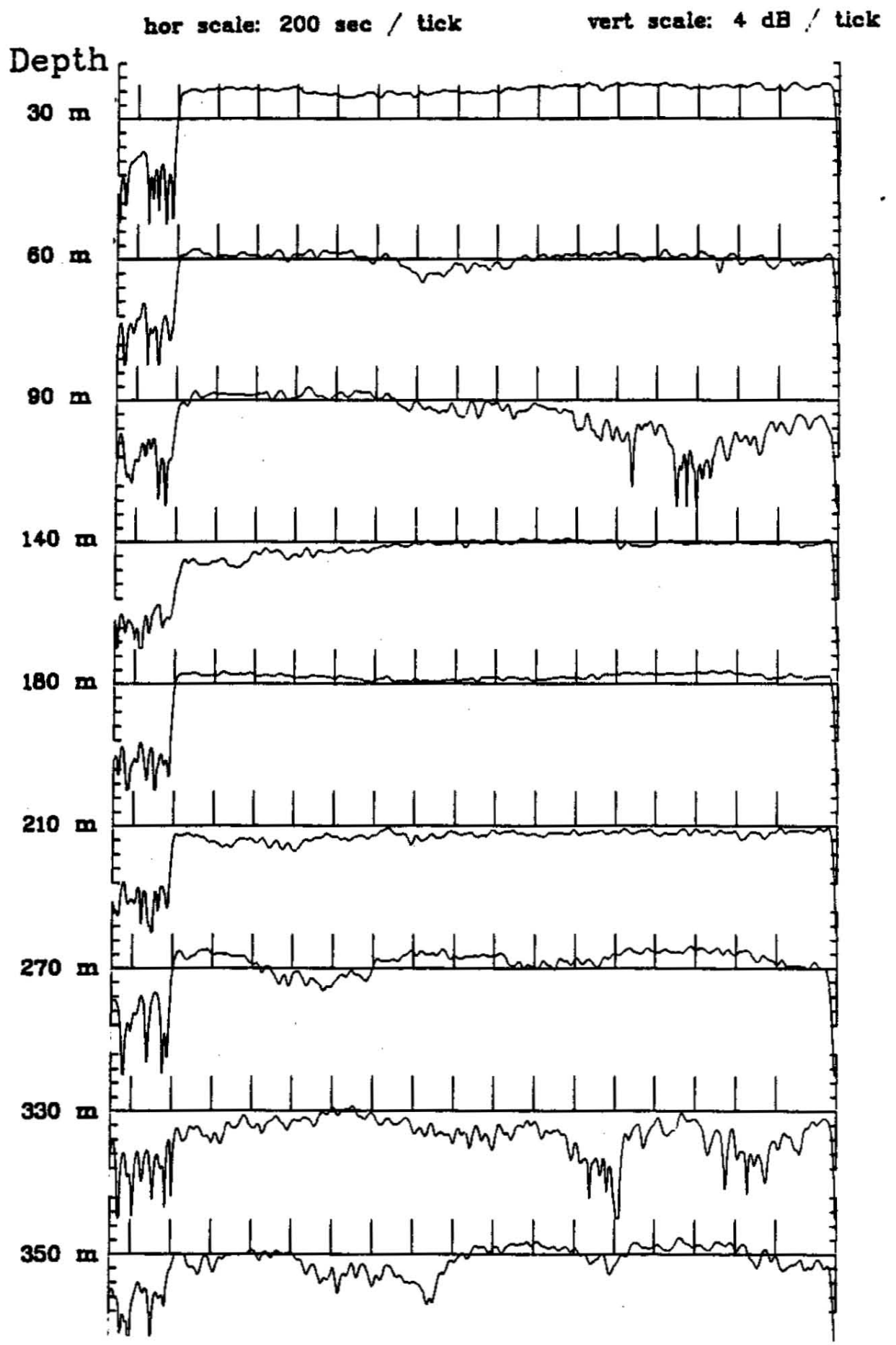

Figure 3-9: Preprocessing Output Time Series - Vertical Array Sensor Magnitudes at $\mathbf{4 7 . 0 0 ~} \mathrm{Hz}$ (part 1 of 2) [180 and $210 \mathrm{~m}$ traces plotted $6 \mathrm{~dB}$ lower than other traces.] 


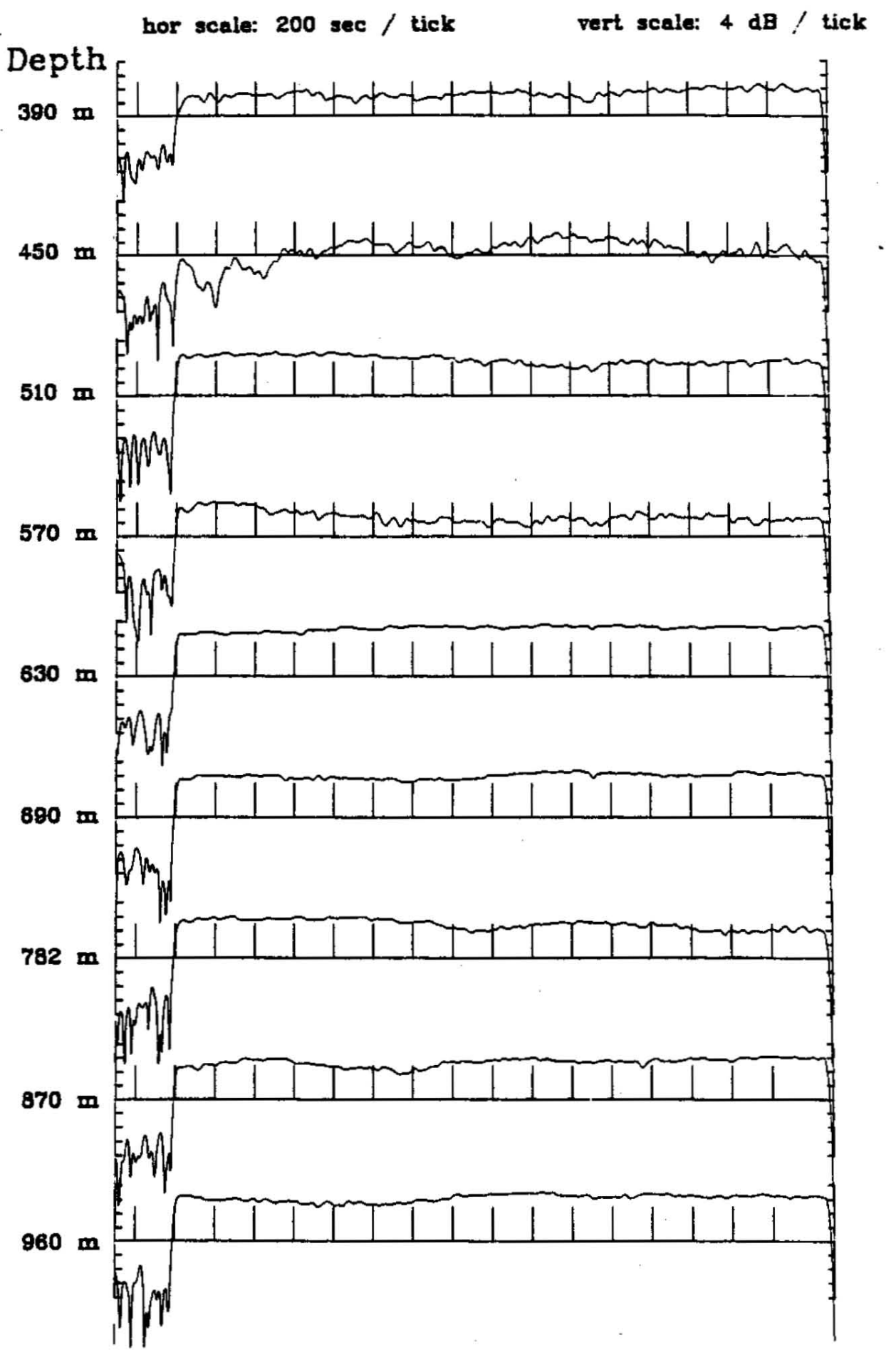

Figure 3-9: Preprocessing Output Time Series- Vertical Array Sensor Magnitudes at $4 \mathbf{7} .00 \mathrm{~Hz}$ (part 2 of 2 ) 


\subsection{An Analysis of the Preprocessed Data}

A great deal of insight can be gained just by studying the preprocessed complex time series of the various vertical array channels. Figure 3-9 displays the log magnitude of the these time series for the $47 \mathrm{~Hz}$ tone from the the vertical array data set, while Figure 3-10 shows the corresponding phase traces for the same signal. Data from all eighteen vertical array sensors are included; the depths of the various sensors are indicated on the left. A $40 \mathrm{~dB}$ per inch scale is used on the log magnitude plots; the phase plots cover the range from $-\pi$ to $\pi$ and display only the principal value of the phase. The time axis spans a range of 65 minutes (3900 sec). The data displayed here in Figure 3-9 and subsequently in 3-11 are both uncorrected for the hydrophone sensitivity, making the amplitude levels only relative. In Chapter 7 , where the data set is studied in much more detail, all amplitude levels presented are absolute and referenced to $1 \mu \mathrm{Pa}$. Note that for the present displays, the decreased sensitivities of the vertical array hydrophones located at 180 and $210 \mathrm{~m}$ cause their levels to appear $6 \mathrm{~dB}$ lower than they actually are.

The signal begins to be seen about 400 seconds into the trace and has the expected duration of 3300 seconds, ending just before the end of of the trace. It is easily distinguished on most of the channels; signal to noise ratios of about $30 \mathrm{~dB}$ (in the $12.5 \mathrm{mHz}$ bandwidth of the final preprocessing filter) are typically observed in the log magnitude traces. The general slope of the phase traces is due to residual mismatch between the received frequency and the demodulator frequency. The negative value of the slope indicates that the demodulation frequency is greater than the received frequency, while the magnitude of the slope is about 1 cycle per hour, corresponding to a mismatch of $0.3 \mathrm{mHz}$. Of this error, $0.1 \mathrm{mHz}$ is due to the difference between the actual demodulator frequency and the nominal soltrer 

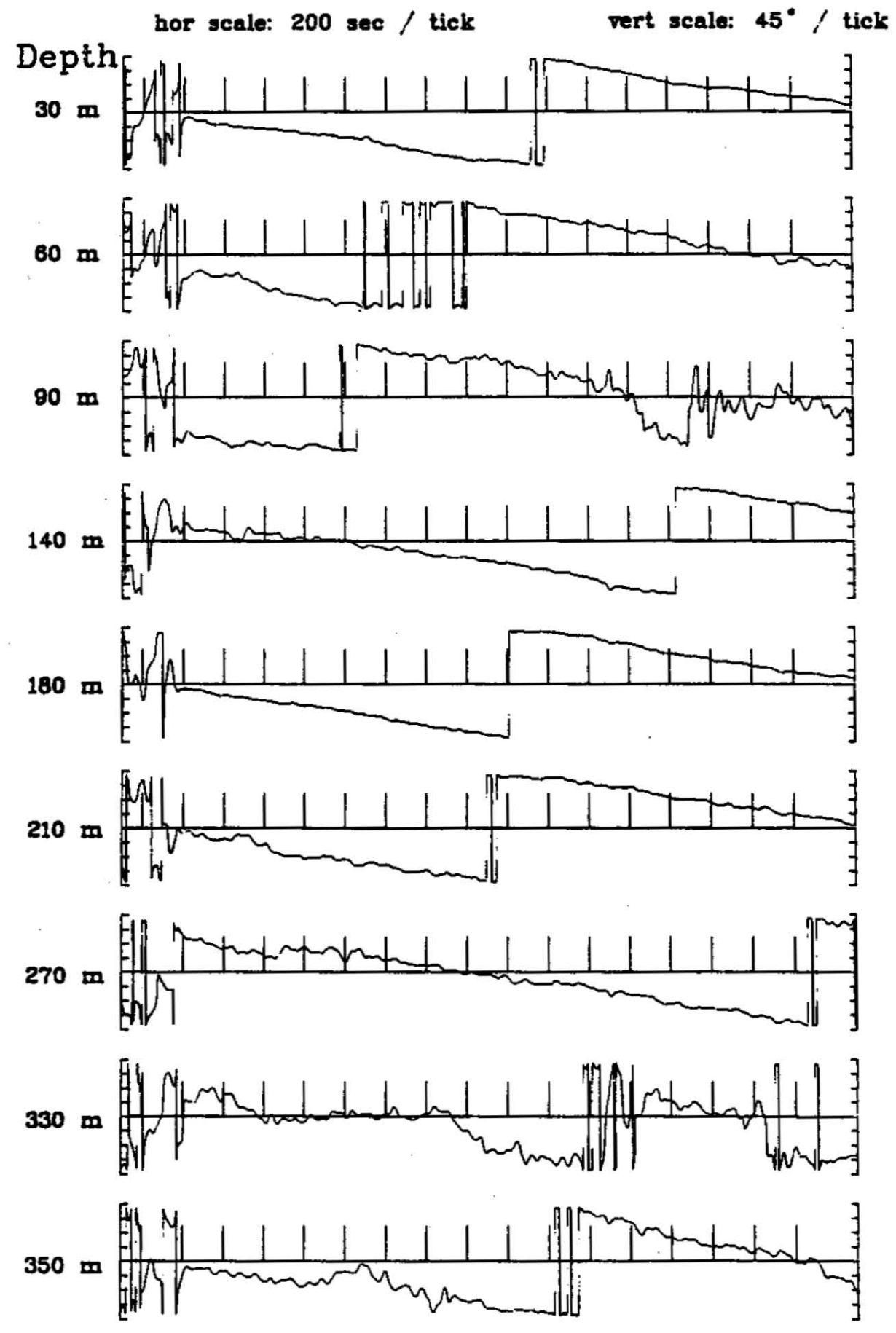

Figure 3-10: Preprocessing Output Time Series - Vertical Array Sensor Phases at $47.00 \mathrm{~Hz}$ (part 1 of 2 ) 

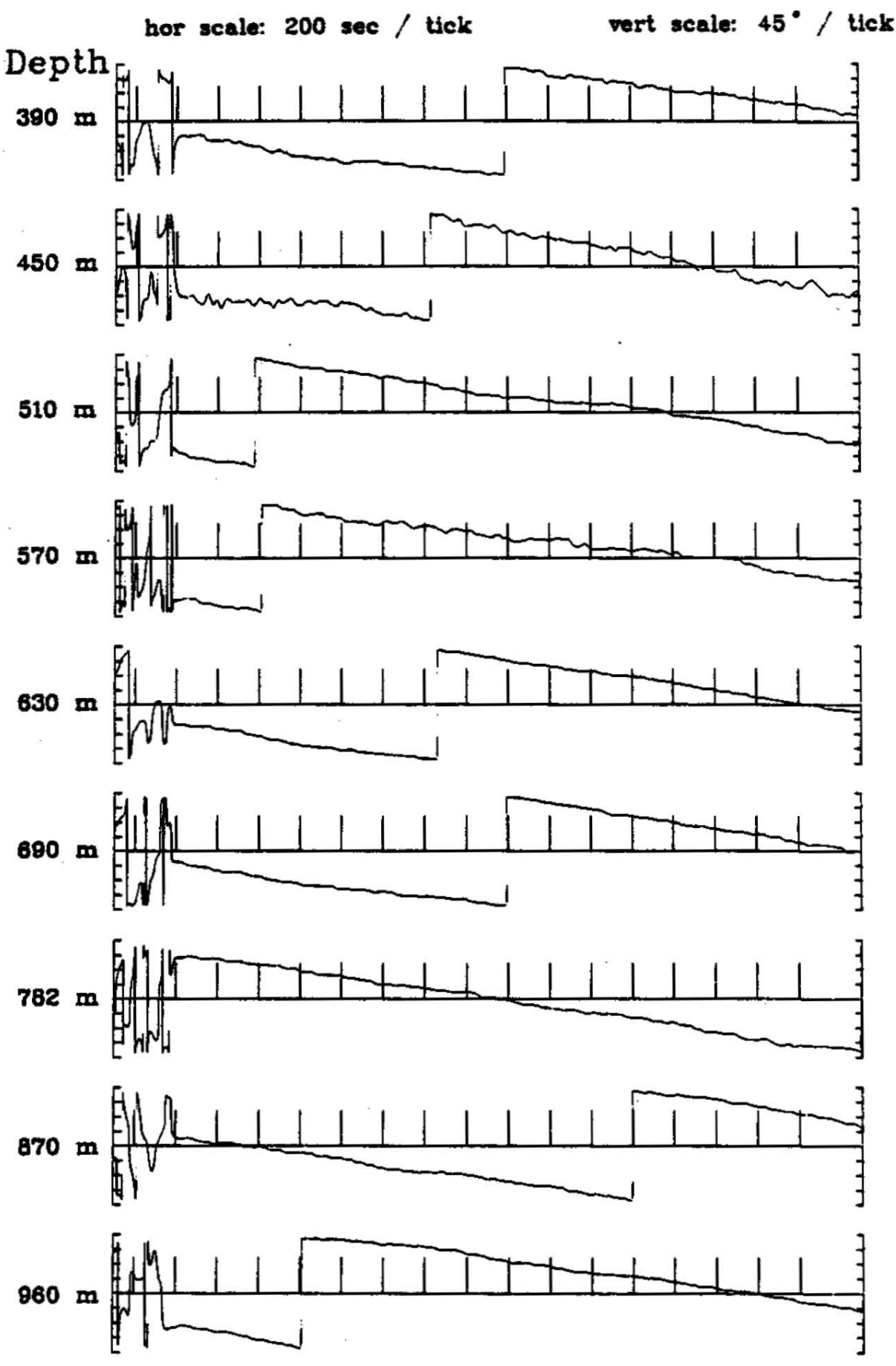

Figure 3-10: Preprocessing Output Time Series- Vertical Array Sensor Phases at $47.00 \mathrm{~Hz}$ (part 2 of 2 ) 
frequency. The remaining $0.2 \mathrm{mHz}$ is attributed to a small amount of Doppler shift caused by source-receiver motion, and corresponds to a relative opening range rate of about $.01 \mathrm{kt}$. The size of this result agrees well with the ice drift speeds experienced at the FRAM camp [78] (no drift speeds were available for the TRISTEN camp). The slight change in phase slope towards the end of the signal indicates that the drift rates were not constant over the full duration of the signil.

In general, the temporal stability of both the log magnitude traces and the phase traces is exceptional. What variations do exist may be broken into short term fluctuations that have time scales of minutes, and longer term fluctuations on the order of tens of minutes. That no significant fluctuations exist with time scales of seconds or smaller is evidenced by the demonstrated ability to coherently process the data in a $12.5 \mathrm{mHz}$ bandwidth without loss of signal power or phase consistency. The short term fluctuations are almost completely negligible on traces of adequate signal to noise ratio, and generally become more pronounced on both types of traces as the SNR decreases. This supports the supposition that the short term fluctuations are probably due to background noise, since the other two likely caluses. temporal channel variations and array movement, would not be expected to show as much dependence on SNR. The cause of the longer term variations; particularly those in the log magnitude traces, is more problematic. One example is the deep fade exhibited on the $90 \mathrm{~m}$ hydrophone over the last third of the signal: other examples of smaller fades can be seen throughout the data. The cause of there is not well understood. They are probably most attributable to channel variations over time, which can be generated in two ways: by actual temporal variations in the physical structure of the channel, and by variations in source-receiver range due to relative ice drift. It is possible that at least some of these variations are related to longer term array movement caused by variations in the effective current incident 
on the array. The change in the signal Doppler noted above is one indication that. this current might not be constant over the full duration of the signal. Navigation data also indicates some changes in drift velocity over the length of the transmission. Theoretically, however, it is difficult to justify the signal level variations observed from just small changes in sensor offset.

While the general quality of both sets of traces is excellent, some noticable exceptions occur. One example is the pair of hydrophones at 330 and $350 \mathrm{~m}$. As above, the poor quality of these traces appears to be caused by the abnormally low signal to noise ratios exhibited on these hydrophones. After a study of all the data. it is difficult to support the hypothesis that these low received signal levels are caused by instrumentation problems. First, just the evidence of coherent (if noisy) phase traces with the proper average phase ramp indicates that these sensors are at. least partially responding to the actual acoustic signal. Additionally, the channeis exhibiting low signal levels tend to be closely grouped in depth, as seen here. Finally, the particular groups of low SNR sensors are frequency dependent: that is. the particular hydrophones exhibiting low signal levels change when the frecurency being transmitted changes. As an example, consider the log magnitude traces exhibited in Figure 3-11 and the associated phase traces illustrated in Figure 3-12. These are identical in format to the data displayed in Figures 3-9 and 3-10. including the channels displayed, but are for the $17.75 \mathrm{~Hz}$ signal from the vertical array data set. Here it is the very lowest sensors in the water column, the two at 870 and $960 \mathrm{~m}$, which display the lack of signal to noise ratio. One must then conclude that these low SNR channels actually represent nulls in the vertical structure of the signal field, caused either by nulls in some dominant mode shape or by the coherent interference of multiple modes that might be present.

The latter hypothesis is strongly supported by another observation that can be 

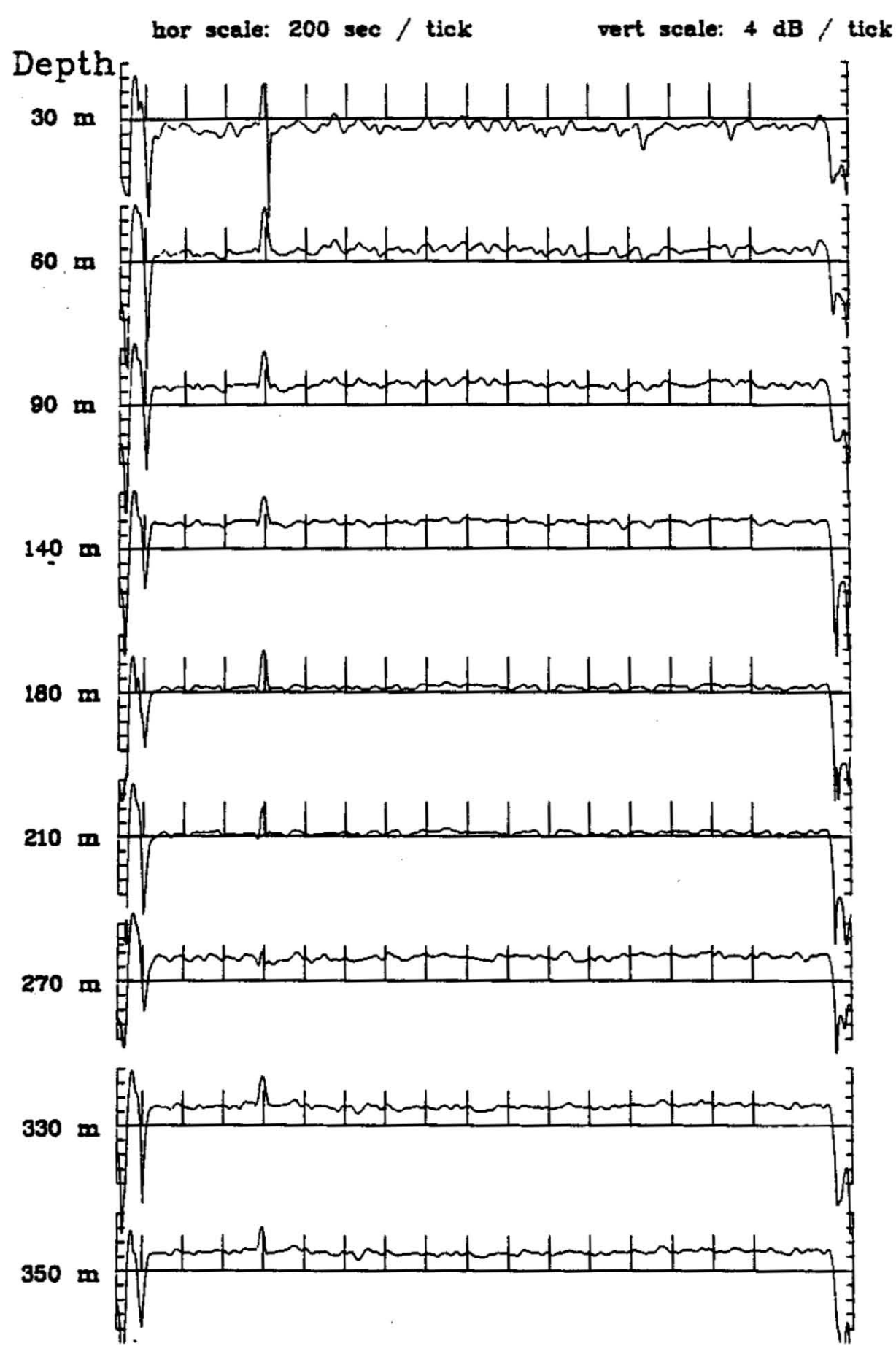

Figure 3-11: Preprocessing Output Time Series - Vertical Array Sensor Magnitudes at $17.75 \mathrm{~Hz}$ (part 1 of 2)

[180 and $210 \mathrm{~m}$ traces plotted $6 \mathrm{~dB}$ lower than other traces.] 


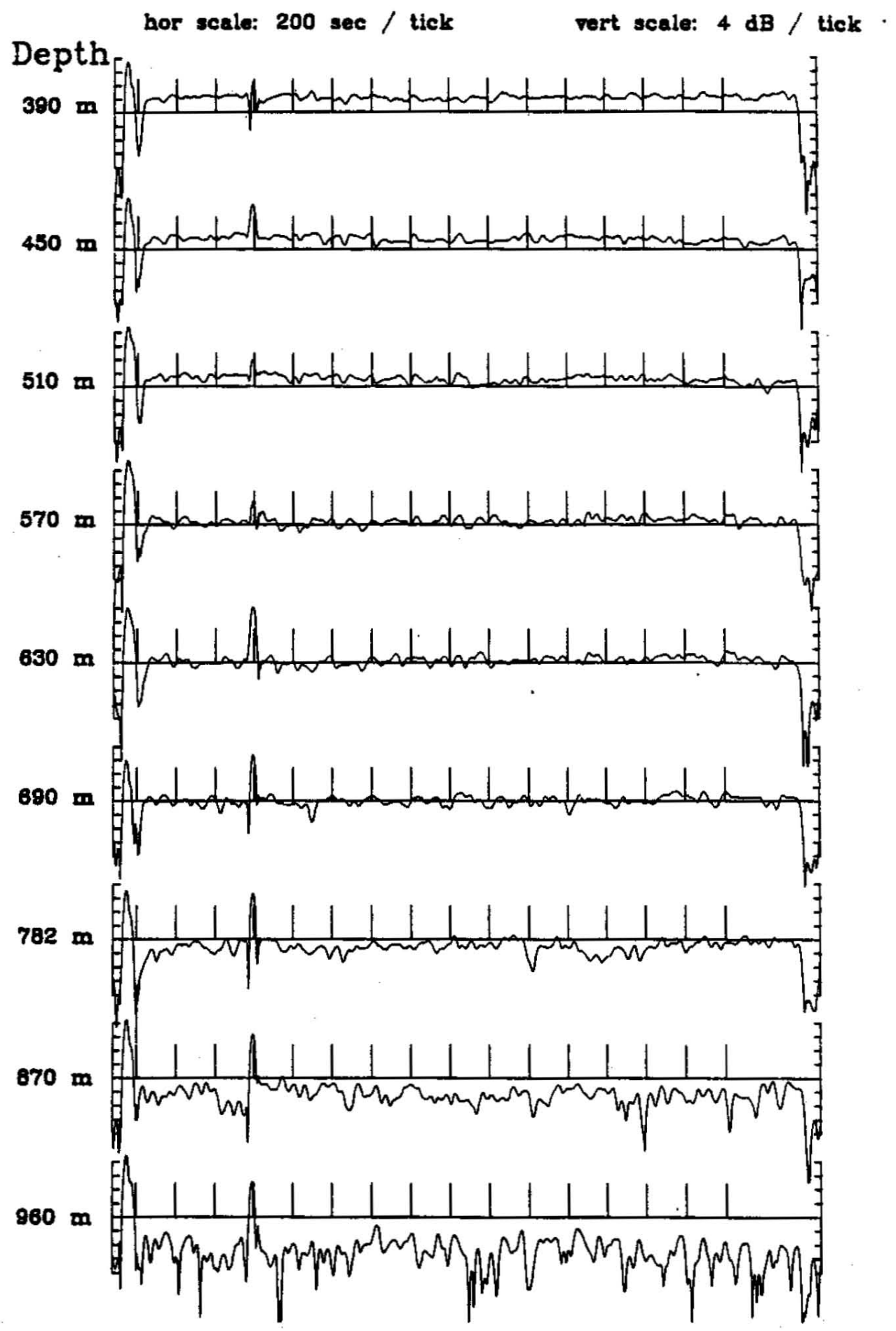

Figure 3-11: Preprocessing Output Time Series- Vertical Array Sensor Magnitudes at $17.75 \mathrm{~Hz}$ (part 2 of 2 ) 
made from this raw data. If a single dominant mode were present and the array were truly vertical, then all the phase traces would exhibit either identical absolute phase, or would be shifted by $\pi$ after going through a null. An easy way to judge the relationship of the absolute phase between sensors is to note the point at which phase wrap around occurs; for two channels to have identical absolute phase. the wrap around must occur at the same point in the trace. The phase traces displayed in Figure 3-10 most certainly do not exhibit either identical absolute phase or $\pi$ phase shifts; moreover, it is very difficult to explain the phase shifts exhibited in terms of array tilt away from the vertical. In order to explain the shifts of just the top three channels, (those at 30,60 and $90 \mathrm{~m}$ ) in terms of array tilt, it is necessary to assume that the local slope of the array near the surface is about $7.5^{\circ}$ in the plane of propagation, about double the value shown in Figure 3-5 and well outside the range of reasonable array tilts. Therefore, it is reasonable to assume that the data displayed in Figures 3-9 and 3-10 include a number of reasonably coherent interfering modes.

For comparison, consider the data displayed in Figures 3-11 and 3-12, which. as mentioned earlier, is the output of the preprocessing for the vertical array data taken at $17.75 \mathrm{~Hz}$. Although the data at first glance is similar to the $47 \mathrm{~Hz}$ diltal just presented, a closer inspection reveals some very interesting differences. First. notice the presence of two strong events, one just before the start of the signal and the other about 600 seconds later. These are known to be blasts from an airgun that was deployed near the FRAM ice camp. Note that the signal phase tracks through the second blast in a reasonable fashion. In general, the signal to noise ratio is slightly lower than that encountered earlier, and the short term variations. particularly those of the phase traces, reflect the increased relative noise level. The frequency mismatch with the demodulator is somewhat higher (about $1.1 \mathrm{mFz}$ ) but 


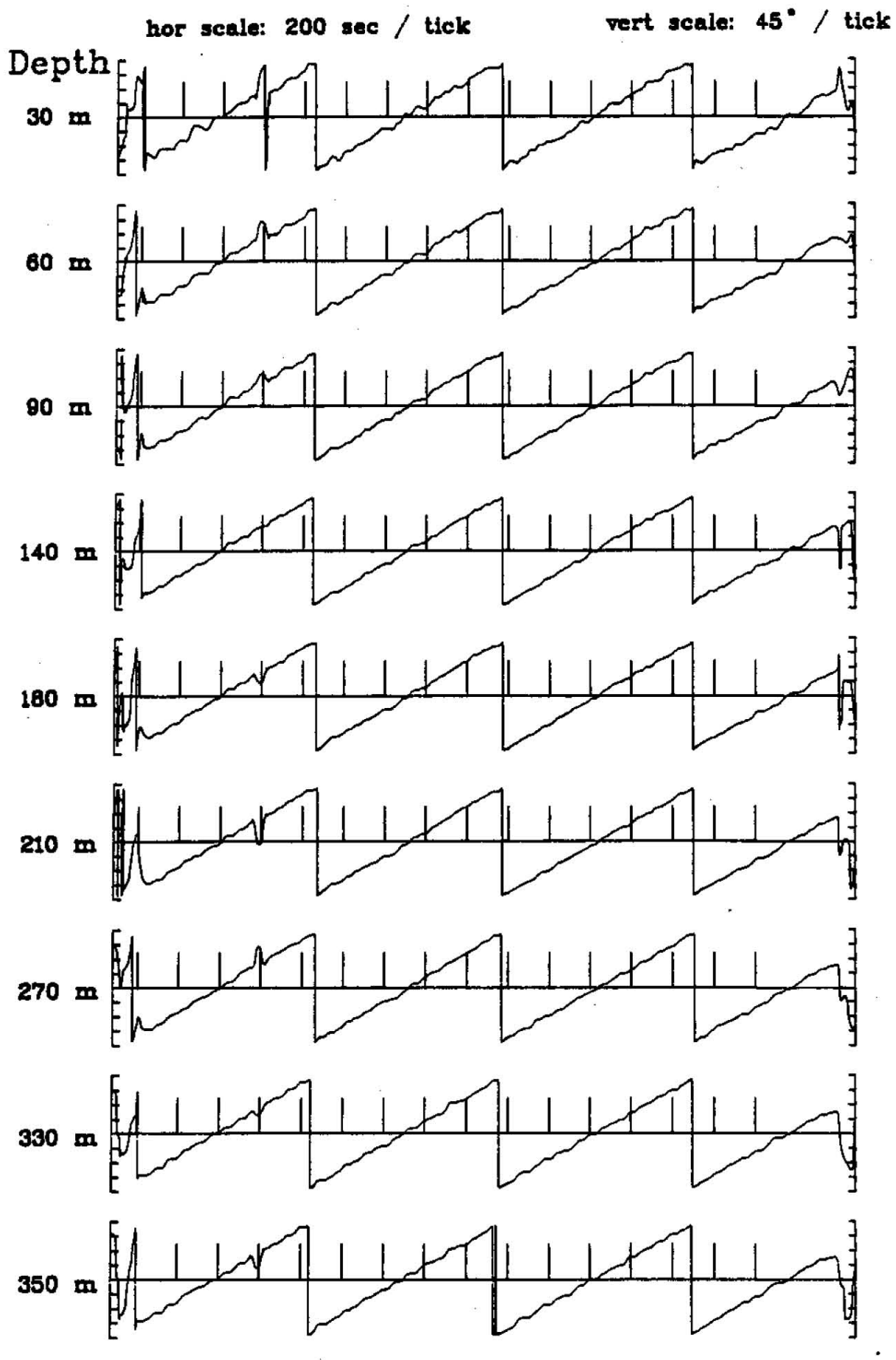

Fi $_{b}$ i. : $\because \because-12$ : Preprocessing Output Time Series - Vertical Array Sensor Phases at $17.75 \mathrm{~Hz}$ (part 1 of 2) 

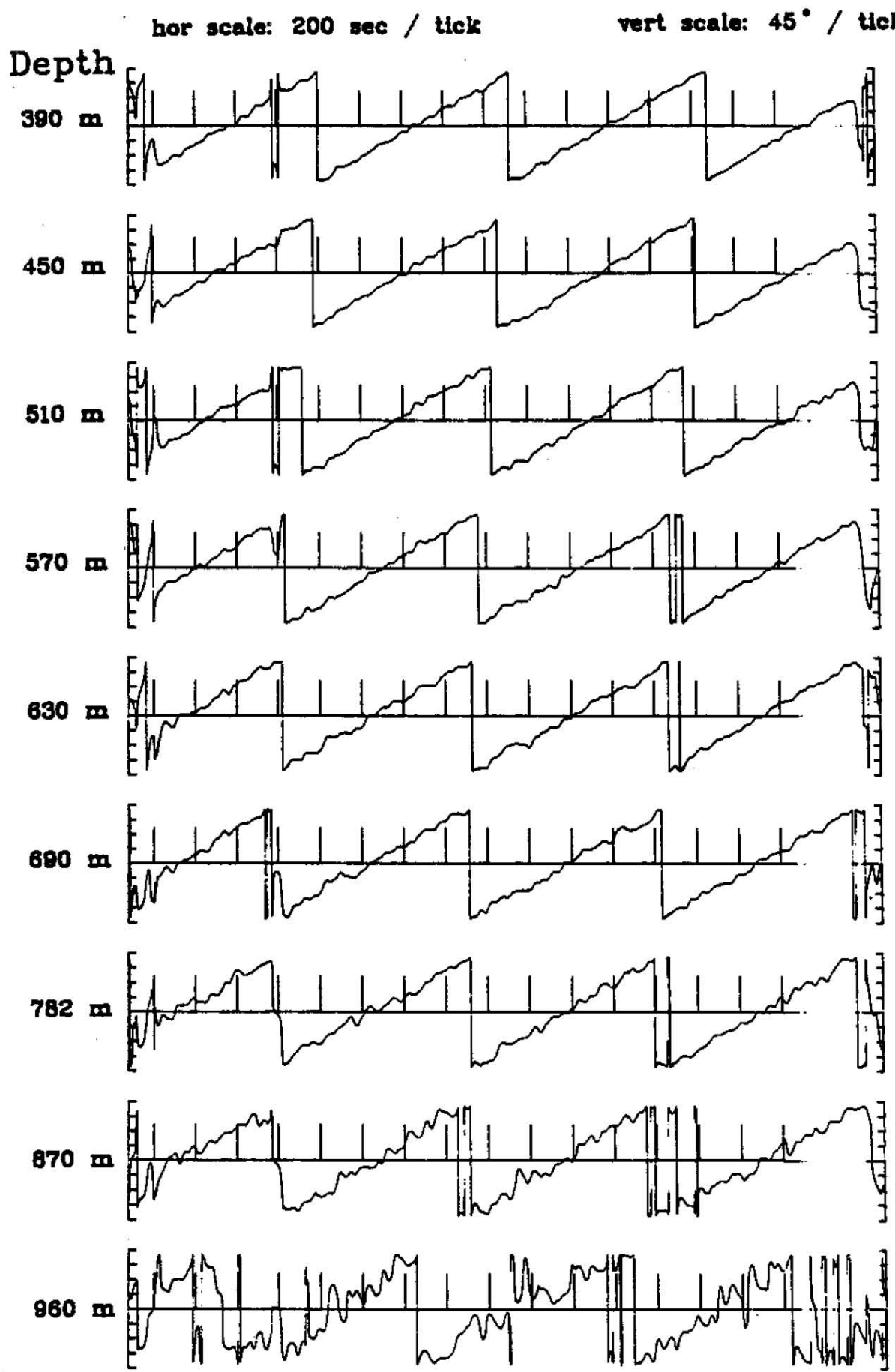

Figure 3-12: Preprocessing Output Time Series- Vertical Array Sensor Phases at $17.75 \mathrm{~Hz}$ (part 2 of 2 ) 
is still well within both the bandwidth of the filter and the range of reasonable Doppler shifts for source-receiver motion. Additionally, the Doppler shift appears to be more constant over the signal duration than that observed previously.

The most important difference between the two sets of data, however, is the way the absolute phases align in Figure 3-12. This is a good example of the absolute phase alignment discussed above, leading to the conclusion that a single mode dominates the vertical structure of the signal at this frequency. Here, the dominant mode must be the first, since no $\pi$ phase shifts (corresponding to sign changes in the mode shape) are observed down the array. Anticipating the results of Chapter 4, the relative levels of the log magnitude plots as a function of depth also indicate a dominant first mode, particularly the low SNR levels of the bottom sensors in the water column (the $870 \mathrm{~m}$ and $960 \mathrm{~m}$ traces); the significant extent of the first mode at $17.75 \mathrm{~Hz}$ is only about $800 \mathrm{~m}$. Note that the traces for the hydrophones at 180 and $210 \mathrm{~m}$ are offset by $6 \mathrm{~dB}$ from the remaining traces, due to their different sensitivities (as indicated in Table 3-III).

The two sets of traces just presented are representative of the outputs of the preprocessing for both the horizontal and vertical array data sets. For all the signals detected, signal to noise ratios fall in the 15 to $40 \mathrm{~dB}$ range for the final filter bandwidth of $12.5 \mathrm{mHz}$, and both stable $\log$ magnitude and phase traces are observed. The short term variations (those on the order of minutes) on both type of traces appear to be related to background noise corruption, since their size appears to vary as the SNR observed at the hydrophone varies. Some longer term signial variations on scales of tens of minutes are observed; while their cause is not well understood, it is possible that array movement is partially responsible, although it is unlikely that this is the sole cause. This leads to the conclusion that the vertical array may be considered stationary for periods of up to 10 or 20 minutes: it can 
probably not be considered constant over periods of an hour or longer, though.

\subsection{Summary}

The FRAM IV Arctic Acoustic Experiment has been summarized, including descriptions of the general locale and arrangement, the scientific objectives, the equipment used, and the data set of interest in this thesis. The data presented here consists of a rich set of tonals in the frequency range from 5 to $71 \mathrm{~Hz}$. These were broadcast from the low frequency source deployed at the TRISTEN ice camp. and then propagated roughly $250 \mathrm{~km}$ to the main FRAM ice camp. Here, they were received on both of the available sensor arrays, and were recorded in a digital format on a $\mathbf{2 4}$ channel acquisition system.

The nature of the two arrays has been discussed in some detail, providing some insight from an engineering viewpoint into the non-ideal aspects of vertical arrays versus those of horizontal arrays. In general, it may be said that the process of designing and implementing a good vertical array is much more demanding than the equivalent development of a horizontal array. In investigating some of the possible difficulties, useful estimates of their various magnitudes for the FR.MI arrays have been made. In particular, maximum sensor offsets of abont $10 \mathrm{~m}$ and $50 \mathrm{~m}$ can be expected for the FRAM IV horizontal and vertical arrays, respectively. For the vertical array, this corresponds to tilt angles of somewhat less than 5 ".

A detailed description of the preprocessing scheme used to compress the data set from more than 1500 Mbytes to a workable size has been provided. This scheme involves two stages, each consisting of quadrature demodulation, filtering with a very narrow FIR low pass filter, and decimation. The output time series resulting from the preprocessing scheme have been demodulated to within $1 \mathrm{mH} z$ of the nominal signal frequency, filtered in a bandwidth of $12.5 \mathrm{mHz}$, and decimated to a 
sample period of $1.6 \mathrm{sec}$. The last filter in the process provides a correlation length of about 20 post-decimated points, leaving approximately 100 degrees of freedom in a typical 55 minute signal.

Two examples of the preprocessing output for the vertical array have been presented and analyzed. Signal to noise ratios of from 15 to $40 \mathrm{~dB}$ are typical in the data set, due to the very narrow filtering of the data. The high SNR leads to nearly ideal log magnitude and phase traces. The demonstrated ability to coherently process the data in the very narrow bandwidth employed without loss of signal power or phase continuity indicates that no significant variations exist with time scales of less than about one minute. Short term fluctuations of one minute order seen in these traces are clearly a function of SNR, indicating that they are probably noise related. Longer term signal variations with periods of tens of minutes are observed; their cause is not well understond. Some, although not all. are likely due to array motion. A more important cause of these variations is probably changes in source-receiver range over time due to relative drift between ice at the two locations. All the observed phase ramps are within the range of Doppler shifts that reasonable values of source-receiver motion would produce.

The absolute phase alignment down the sensors of the vertical array provide a great deal of insight into the underlying modal structure. For the $47 \mathrm{~Hz}$ data of Figures 3-9 and 3-10, this phase alignment implies a number of significant modes probably interfering coherently, since nulls in the vertical structure of the field are visible. For the $17.75 \mathrm{~Hz}$ data of Figure 3-11 and 3-12, however, a single mode, the first, appears to dominate the vertical structure. A study of the pattern of received amplitudes versus depth for the $17.75 \mathrm{~Hz}$ data supports the same conclusion. 


\section{Chapter 4}

\section{The Modal Structure of the Arctic Sound Channel}

The purpose of this chapter is to characterize the aspects of the central Arctic environment that impact the propagation of sound; to utilize that characterization to study the modal structure of the channel in which the FRAM IV experiment was conducted; and to examine the interaction between the modes and the source and receiver geometries present during the experiment. These results are directly needed for the work that follows, but also allow some more general observations to be made. Typically, such a study would be employed only in the interpretation and modeling phases of an experiment. In the present effort, however, the modal decomposition techniques to be developed all require both the shapes and the associated horizontal phase speeds of the significant modes to be known a priori. Prediction of the channel's modes thus assumes a more important role here, that of a necessary preliminary to the beamforming implementation.

The requirement for prior knowledge of the local modal structure has important theoretical and practical consequences. Among the most significant is that a whole range of modeling and prediction issues now have a direct impact on the performance of the beamformer. From a theoretical viewpoint, questions of processing performance become dependent upon the assumptions made about the environment in which the array is deployed. More practical issues include the adequate in situ measurement of the local sound speed profile and methods for estimating other needed environmental parameters. Techniques for computing the mode shapes and horizontal phase speeds must also be addressed. Since this 
involves solution of the one-dimensional Helmholtz equation (2.7), usually by numerical or other approximate methods, the applicabilty, stability, and accuracy of the method chosen must be considered. The question of the channel's gross temporal stability becomes important since dynamic updates of the modal data may be necessary. These issues all affect beamforming performance to the extent that they generate mismatches between the assumed and actual modal structures: the mismatches then skew the results of the modal decomposition in proportion to their severity.

The starting point for this investigation is a compilation of the environmental data necessary to describe the acoustic channel. The most important element is a knowledge of the average water column sound speed profile at the receiver: additional useful information includes descriptions of the bottom and overhead ice cover as well as an understanding of the spatial and temporal variations of the channel profile. The method of mode generation to be employed is then discussed and the results of its application to the central Arctic sound channel summarized. Finally, these results are analyzed so that the impact of various environmental features on the modal structure can be assessed.

Coachman and Aagard [19] provides a good general reference for Arctic oceanography. Two works by Chen [15], [16] provide more detailed discussions of Arctic sound speed profiles directly applicable to the data set of interest. Some work on the modal structure of the central Arctic has been done previously: for comparison with the results shown here, the reader may consult Duckworth [29] or Polcari [67]. No significant variation of results is found among the three. Another good reference is Kutschale [48], while Mellen [54] and Yang and Giellis [84] both provide additional discussion. 
$-91-$
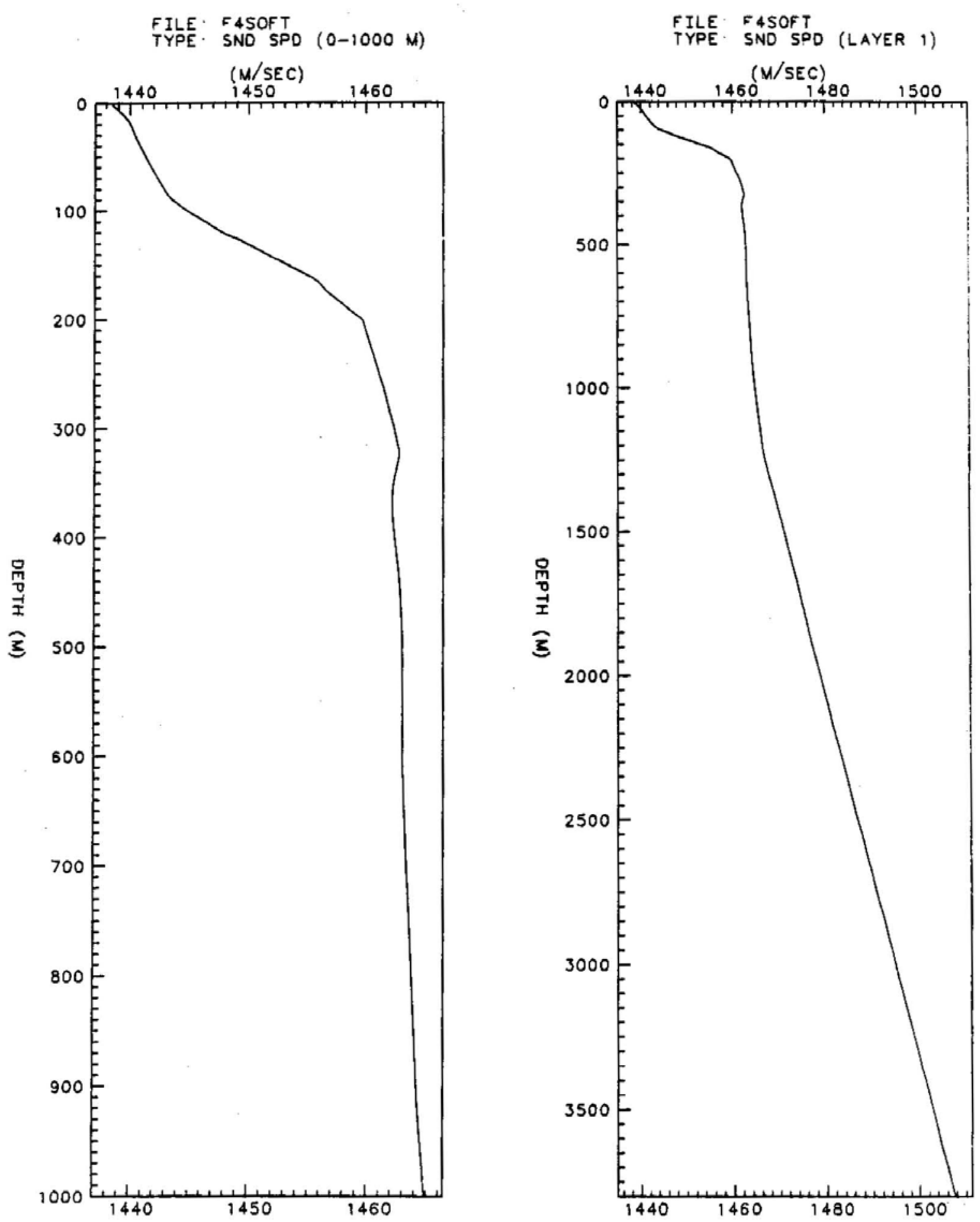

Figure 4-1: Sound Speed Profile of the Water Column at the Receiving Arrays 


\subsection{A Representative Sound Speed Profile from the Central Arctic Ocean}

Figure 4-1 indicates the sound speed profile on which the following modal calculations are based. The left hand plot shows the detail of the upper $1000 \mathrm{~m}$, while the right hand plot shows the complete water column. This profile represents the best average estimate of sound speed at the FRAM receiver camp during midApril, 1982, and is identical to the profile presented in Polcari [67].

As is found throughout the Arctic, the sound speed is essentially a monotonic increasing function of depth. Within the uppermost regions (down to about $150 \mathrm{~m}$ ). the controlling mechanism is not the water temperature, as is typically the case for most open ocean areas, but rather the salinity gradient generated by the presence of the overhead ice canopy [15]. The variation in salinity provides a change of about 5 $\mathrm{m} / \mathrm{sec}$ in sound speed across the first $100 \mathrm{~m}$. By comparison, temperature variations account for about $1 \mathrm{~m} / \mathrm{sec}$ of change in this zone. Below the $150 \mathrm{~m}$ point, the temperature takes over as the dominant factor, resulting in an almost isovelocity profile to roughly $700 \mathrm{~m}$. The deep regions (below $800 \mathrm{~m}$ ) are primarily pressure controlled, as is the case with deep ocean profiles in more temperate climates.

The net effect of the combination of salinity and temperature variations is to produce a very strong surface duct encompassing about the upper $200 \mathrm{~m}$ of the water column. As will be shown, this surface duct plays a significant role in determining both the modal structure of the Arctic channel and the nature of the propagation there. An average slope of the deeper portion of the central Arctic profile (that below $200 \mathrm{~m}$ ) can be taken as $0.013(\mathrm{~m} / \mathrm{sec}) / \mathrm{m}$, not far different from the sound speed pressure dependence of $0.016(\mathrm{~m} / \mathrm{sec}) / \mathrm{m}$. By contrast, the mean gradient in the surface duct is $0.13(\mathrm{~m} / \mathrm{sec}) / \mathrm{m}$, about ten times larger.

Figure 4-2 details the sound speed profile used to model the Arctic buttom. 

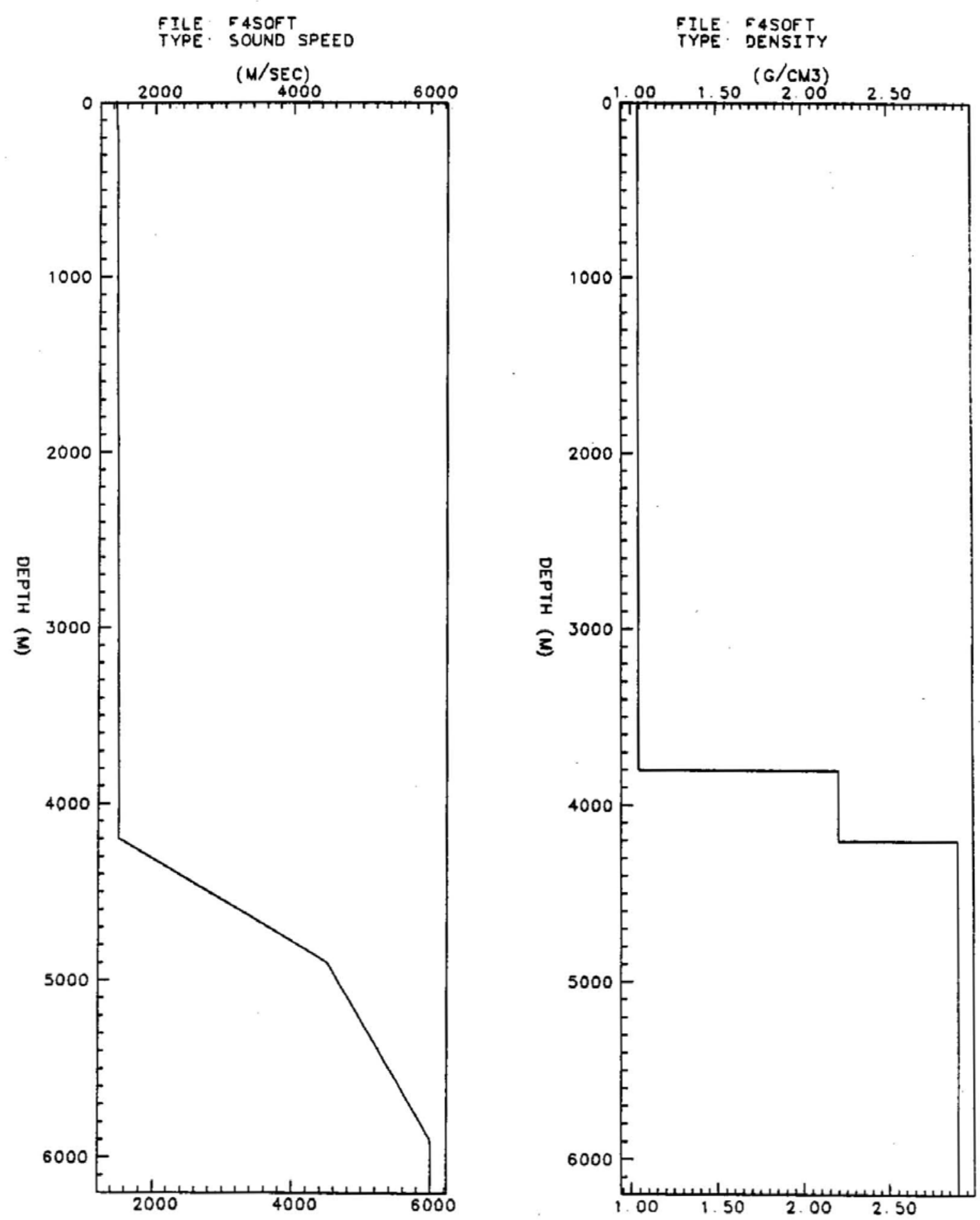

Figure 4-2: Assumed Bottom Characteristics at the Receiving Arrays 
The main features here have been chosen based on the composite profile presented in Duckworth and Baggeroer [30]. The bottom is modeled as four layers. A $400 \mathrm{~m}$ sediment layer of density $2.2 \mathrm{~g} / \mathrm{cm}^{3}$ extends to $4200 \mathrm{~m}$ from the assumed bottom depth of $3800 \mathrm{~m}$, reaching a sound speed of $2.3 \mathrm{~km} / \mathrm{sec}$. Two layers are then used to transition at a depth of $5900 \mathrm{~m}$ to a soft basement capable of propagating sound. The sound speed of this basement is assumed to be $6.0 \mathrm{~km} / \mathrm{sec}$, while its density is assumed to be $2.9 \mathrm{~g} / \mathrm{cm}^{3}$.

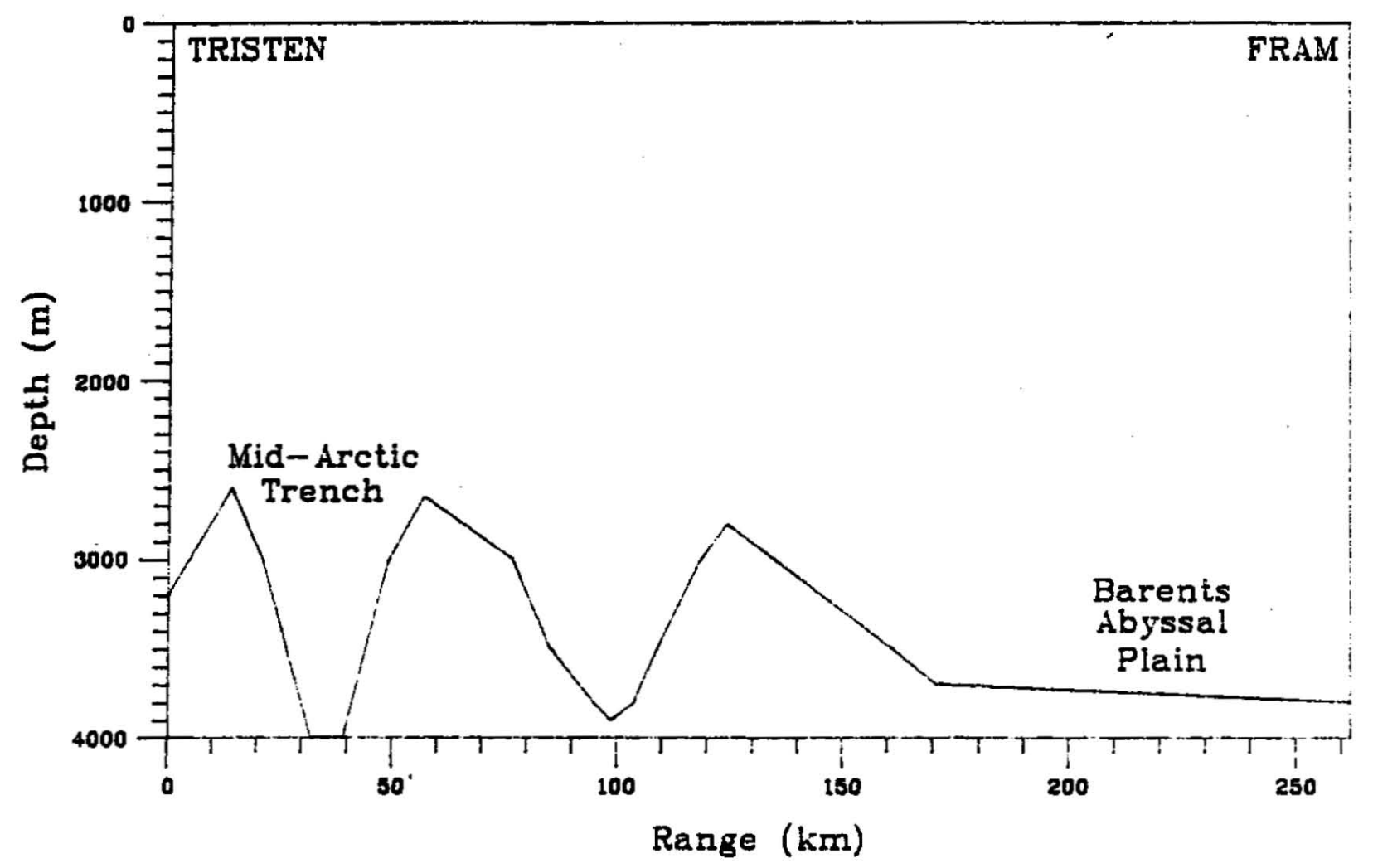

Figure 4-3: Historical Bathymetry along the TRISTEN/FRAM Propagation Path (taken from [14])

[Note different range and depth scales.] 


\subsection{Temporal and Spatial Sound Speed Variations}

The sound speed profile of the central Arctic has been shown to be unusually stable in both time and space [16]. This is due to a number of factors, the most significant being the geographical nature of the area and the year-round continuous ice canopy. The isolation of the central Arctic limits variations due to external influences, while the ice cover insulates the ocean below from the solar and wave effects found elsewhere. Figure 4-1 can therefore be taken as representative of the profile that would be encountered anywhere along the transmission path throughout the length of the experiment. Some range variation in the sound speed profile c:an be expected due to large scale oceanographic features such as eddy systems, though.

Although the water column is spatially well behaved, the same conclusion cannot be drawn of the bottom. Figure 4-3 indicates the bathymetry along the propagation path, based on charted data (in situ measurements were not available). The TRISTEN source camp was located almost over top of the Mid-Arctic Ridge. which is easily identified in the figure from the central trench structure at a range of $40 \mathrm{~km}$. The series of peaks on either side of this trench extend to the $2500 \mathrm{~m}$ depth range. The severe range dependence of the bottom significantly affects the nature of the propagation, as has been graphically illustrated by the comparison of shot data with that taken from earlier experiments [3]. Any mode that interacts significantly with the buttom in the region of the ridge can be expected to be attenuated to such an extent that its contribution to the total sound field at the receiver would be small; thus, modes having turning points much below 2000 in are probably negligible for the TRISTEN/FRAM propagation path. 


\subsection{The Acoustic Impact of the Ice Cover}

Much of the Arctic's uniqueness from an acoustic viewpoint is due to its relatively continuous year-round ice canopy. Both ice camps were located well within the zone of continuous ice coverage. At times (especially in the late summer), significant regions of open water can occur even in the area nominally designated as continuously covered. However, this effect is not overly significant during the early spring. The assumption of continuous ice cover over the full extent of the propagation path is therefore reasonable.

The influence of the ice canopy on the propagation path is a significant effect which is not yet well understood. An excellent reference that reflects the current thought on the subject is a recent memorandum by DiNapoli and Mellen [25]. The primary effect of the ice cover at low frequencies is to increase the effective attenuation coefficient by about two orders of magnitude over values found in open water. Prediction of such a loss falls into the general category of rough surface scattering. A number of different models of varying complexity may be applicalble. ranging from a simple rough free surface to a full solid layer model, including she:ar wave effects. To date, none of the models investigated shows acceptable agreement with experimental data. The most important parameters needed to describe the ice canopy are the mean ice draft and the rms roughness, correlation length. and power spectrum of the random surface that forms the ice-water interface. Typical values reported by DiNapoli and Mellen for these parameters are a mean draft of $4 \mathrm{~m}$, with an rms roughness of $2 \mathrm{~m}$ and a correlation length of $44.8 \mathrm{~m}$. These results are in reasonable agreement with earlier work published by Wadhams [83]. DiNiapoli and Mellen also provide an analytical fit to the experimentally measured ice-water interface power spectrum. They give the two-dimensional power spectrum as 


$$
P(\vec{\kappa})=\frac{4 \pi(2 \sigma / L)^{2}}{\left[(2 / L)^{2}+(2 \pi|\vec{k}|)^{2}\right]^{2}},
$$

assuming an isotropic random surface. Here $\vec{\kappa}$ is the two-dimensional wavenumber vector, $\sigma$ is the rms roughness of the surface, and $L$ is the correlation length.

In this thesis, the ice canopy is modeled by a rough free surface at the icewater interface, more for simplicity than any other reason. While the roughness hals an important direct effect on modal attenuation, its impact on the gross properties of the modes (the mode shapes and their horizontal phase speeds) is determined by its effect on the mean channel profile. Within the limits of the model, the position of the mean free surface is displaced by the amount of the mean ice draft $(4 \mathrm{~m})$. which is probably insignificant when compared to other sources of depth error in the profile. Ice effects have therefore been ignored in the sound speed profile usecl to generate the mode parameters.

\subsection{Solutions of the Helmholtz Equation for the Mode Shapes}

To convert the environmental information presented above into mode shape and phase speed data, one must solve the one-dimensional Helmholtz equation eigenvalue problem (2.7) for the specified sound speed profile at each desired frequency. The form of equation (2.7) assumes that all layers, including the propagating basement, are fluids; shear wave propagation in the bottom is implicitly ignored. In the sequel, it is shown that neither the mode shapes nor their associated horizontal phase speeds are significantly influenced by the bottom characteristics. Thus, the neglection of bottom shear effects is a reasonable approximation. All attenuation effects are also ignored here, as they do not affect the shapes and phase speeds of the modes to any great extent. 
One method for finding the mode shapes and phase speeds is to employ the WKB approximation in solving the Helmholtz equation. This approach is questionable at the low frequencies of interest here. Another technique involves the use of propagator matrices, but this method is more applicable to a discretely layered channel model than it is to the continously varying profile of Figure 4-1. The most accurate method for the present circumstances is a shooting technique. involving direct numerical integration of equation (2.7). The integration is initialized to meet one of the boundary conditions, and the assumed eigenvalue is varied until the other boundary condition is met. Layers of varying density can be accommodated through the proper application of cross-boundary continuity conditions (given in Figure 2-1 as the propagating bottom boundary conditions). The mode's horizontal phase speed is recovered from the eigenvalue, while the eigenfunction, when properly normalized to meet equation (2.9), yields the mode shape.

The main drawback of this approach is the instability of the numerical integration in the evanescent regions of the mode shape. Since equation (2.7) is second order, it supports two possible solutions, one exponentially increasing and the other exponentially decreasing in the direction of integration. Numerical errors that excite the increasing solution can quickly dominate the desired solution, even though they are insignificant when introduced. The Arctic profile, with its monotonic increasing sound speed, is particularly susceptible to this effect, since the mode shapes in this instance typically exhibit extensive evanescent regions.

To circumvent this problem, a modified integration method developed by Baggeroer [4] has been employed in generating the mode shapes presented here. The Helmholtz equation is first cast into a state variable form of second order. Treating the state variables as a rectangular Cartesian pair, the problem is then 
transformed to a magnitude/phase pair of equivalent polar coordinate variables. The differential equation for the magnitude variable decouples from the equation for the phase variable and the boundary conditions take on rather simple forms, so that only the first order differential equation for the phase need be solved via shooting to obtain the eigenvalue. Then, given the phase solution, the mode shape is recovered by integrating for the magnitude and converting back to the original variables. The development by Baggeroer includes a series of conclusions about the proper directions of integration for numerical stability. This approach has also been successfully employed by Duckworth [29].

To ensure the greatest possible accuracy, the Baggeroer approach has been implemented here with a series of fourth order Adams-Bashforth predictor-corrector integrators [12]. A depth grid of 5000 points is employed for the integrations. providing a sample interval of $1.2 \mathrm{~m}$. The resulting mode shapes have been normalized for the pressure field (rather than the velocity potential field) in accordance with equation (2.9). As a test of the internal consistency of the algorithm, the orthogonality integral of equation (2.8) has been computed for different mode pair combinations at several frequencies of interest. Typical integration results are of the order of $10^{-4}$, with the worst errors generally occurring either when the pair consists of neighboring modes or when the first mode is one of the pair. However, even in these conditions results as large as $10^{-3}$ are obtained only rarely. 


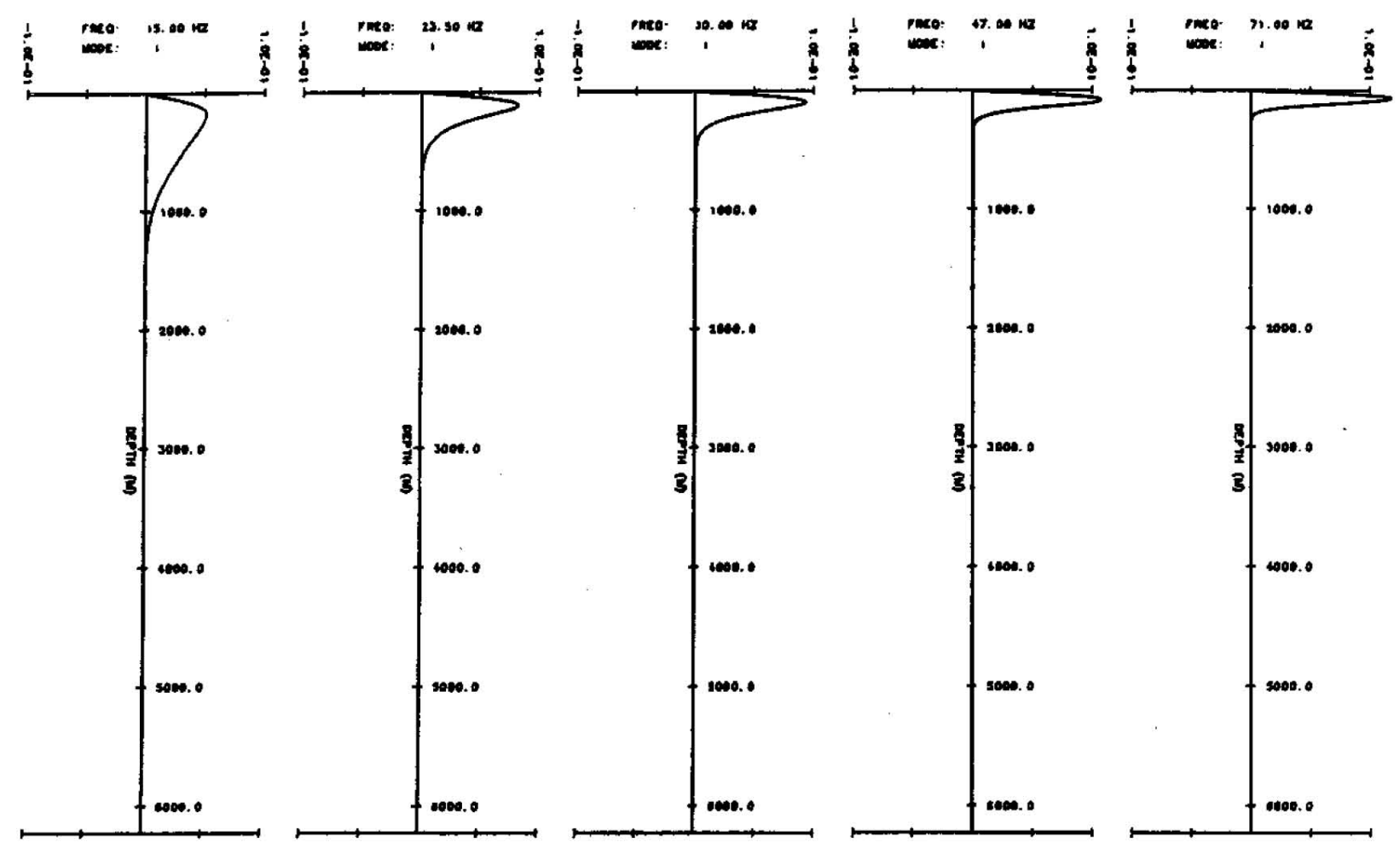

Figure 4-4: Shape of the First Mode at Various Frequencies

\subsection{An Analysis of the FRAM IV Modal Structure}

All modes with turning points above the nominal bottom depth of $3800 \mathrm{~m}$ have been computed at the frequencies available in the vertical array data set the nominal sound speed at $3800 \mathrm{~m}$ is $1508 \mathrm{~m} / \mathrm{sec}$ ). Table $4-\mathrm{I}$ indicates the number of modes found at each frequency, as well as the number with turning points above $2500 \mathrm{~m}$; as discussed above, the modes contributing significantly to the sound field at the receiver are all probably included in this last group. Figures $t-4$ through $4-\pi$ display the shapes of the first four modes at some of the frequencies for which data is available. The frequencies chosen for display were selected so as to span those 


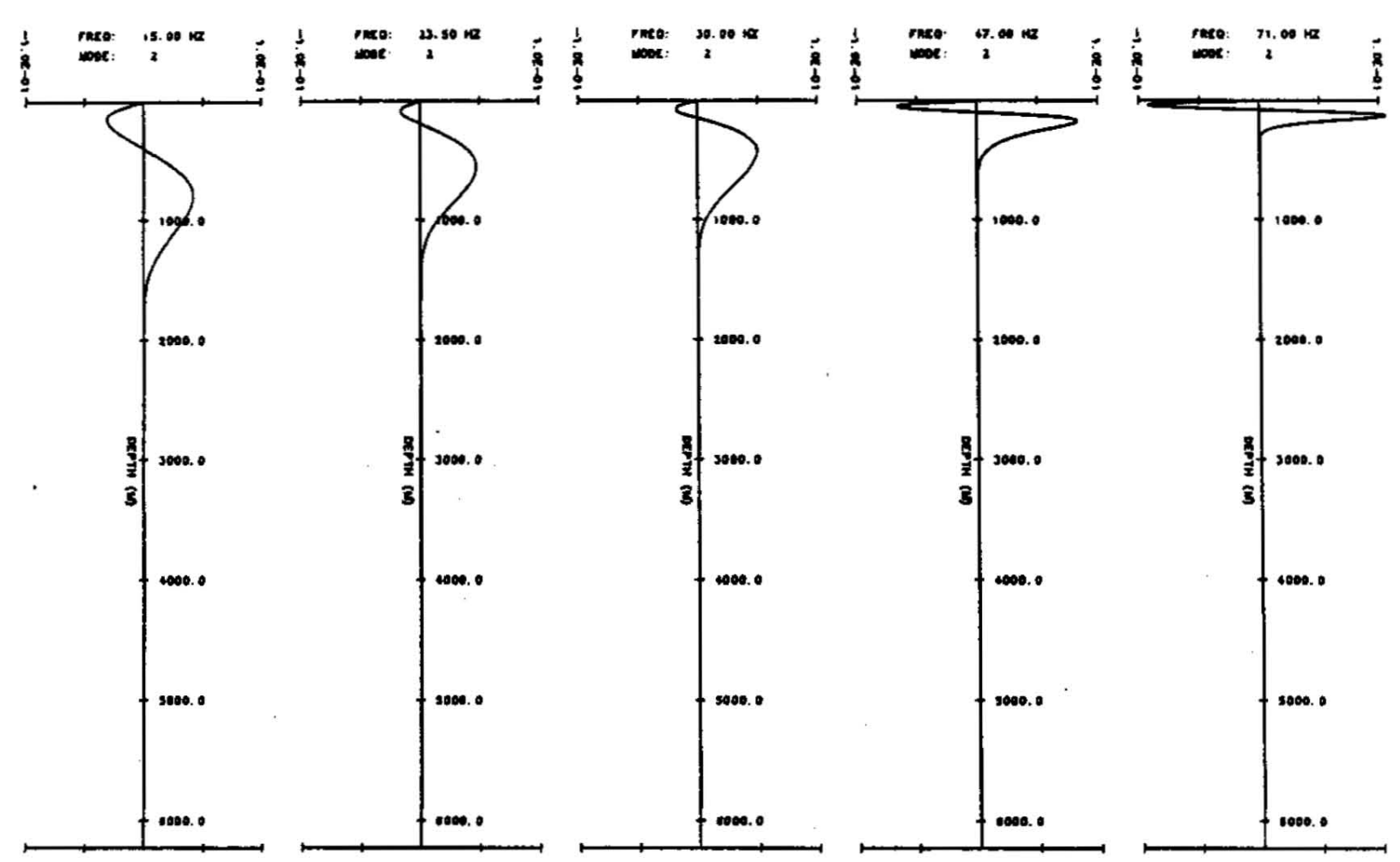

Figure 4-5: Shape of the Second Mode at Various Frequencies

frequencies of interest that are not shown.

\subsubsection{Mode Shape Analysis}

The extent of a particular mode is inversely related to frequency. Higher order modes look similar to the fourth, but extend further into the water column with a greater number of sign changes. Their general shape is not far different from that of Airy functions. In all cases, the absolute maximum of the mode shape occurs in the half cycle immediately above the mode turning point.

As both the source and the horizontal receiving array were deployed to a depth of $91 \mathrm{~m}$, the values of the various mode shapes there are of particular 


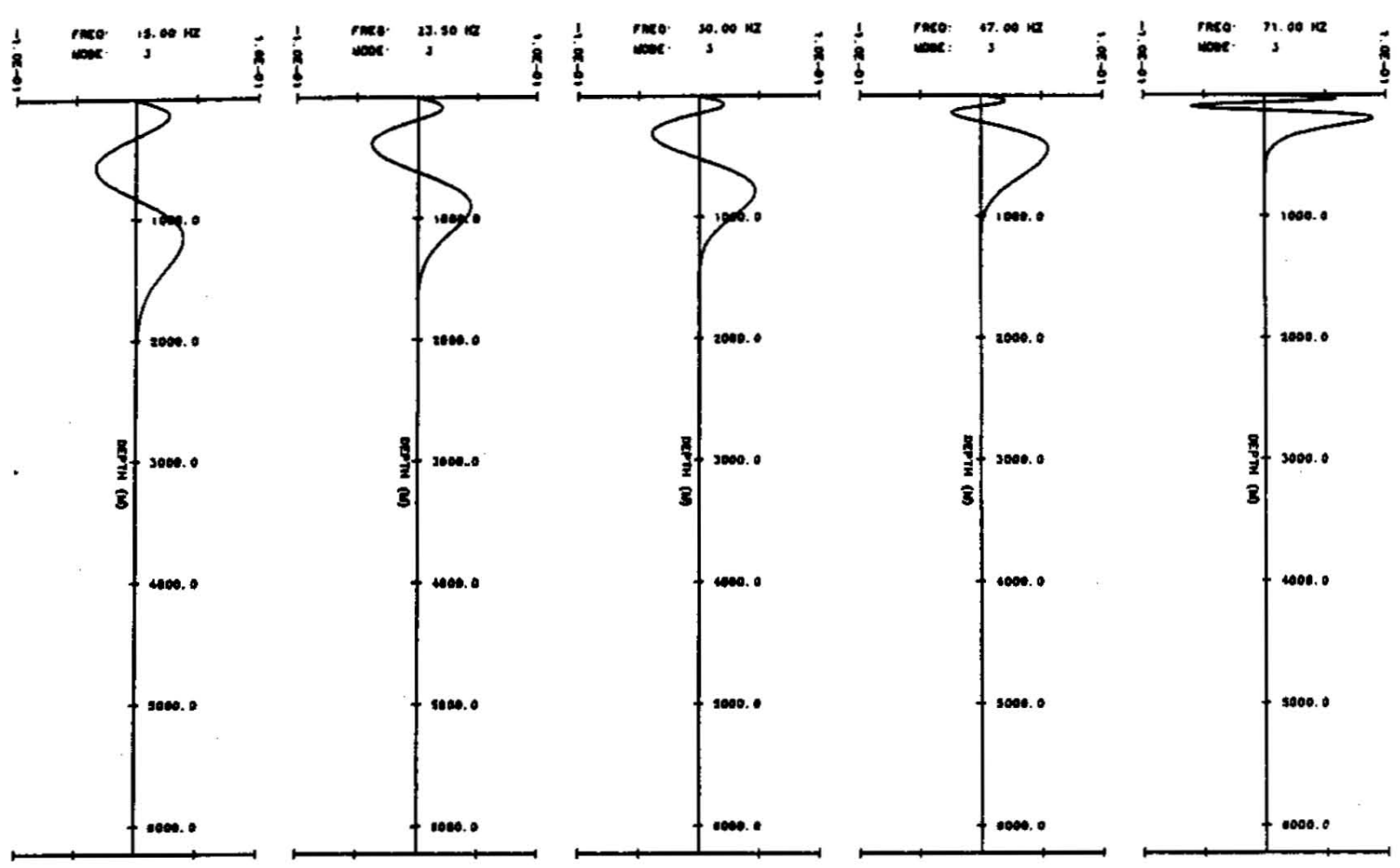

Figure 4-6: Shape of the Third Mode at Various Frequencies

interest. Figure 4-8 indicates the levels of the mode shapes in $d B$ at $91 \mathrm{~m}$ for the first 10 modes at 15, 30, 47, and $71 \mathrm{~Hz}$. As indicated in Chapter 2, it is these levels which control the distribution of source energy in the various modes. Figure $4-8$ shows that, at its $91 \mathrm{~m}$ deployment depth, the TRISTEN source drives the the first mode much better than any other mode. The higher order modes, while excited less efficiently than the first, are all driven about equally; moreover, the level of this plateau appears to be insensitive to frequency. The $47 \mathrm{~Hz}$ curve is something of an anomaly, in that at this frequency modes 2 through 6 all exhibit nulls very close to the source depth (see Figures $4-4$ through $4-\overline{7}$ ); this accounts for the unustally low levels of modes 2, 3 and 4 . However, the higher order modes at $47 \mathrm{~Hz}$ still appear 


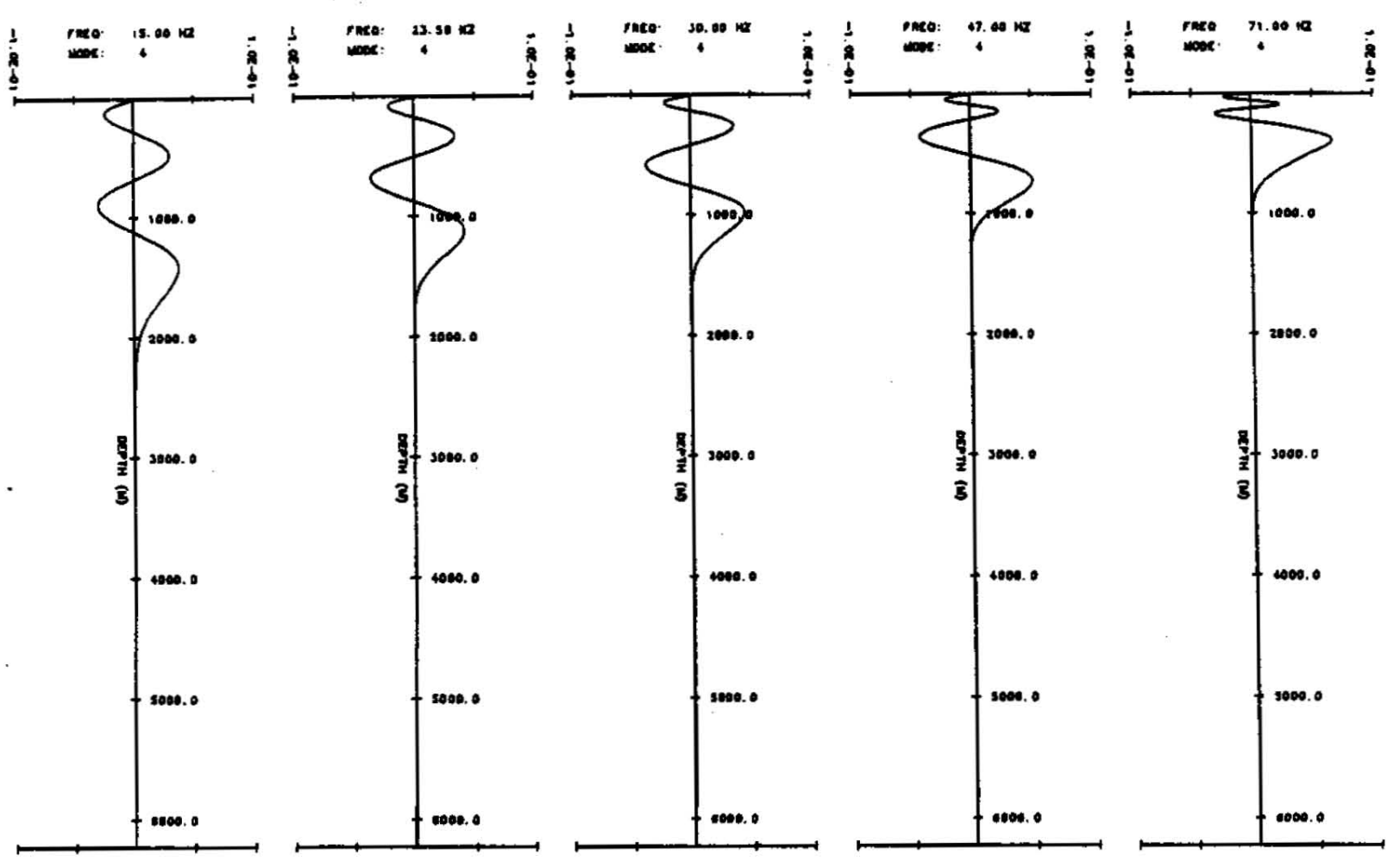

Figure 4-7: Shape of the Fourth Mode at Various Frequencies

to plateau at about the same levels as those of other frequencies.

Figure 4-8 may also be interpreted in a different context. Because the horizontal array at the FRAM receiving camp was deployed to the same $91 \mathrm{~m}$ depth as the source, the discussion above applies equally well to the relative sensitivity of the horizontal array elements to energy carried by the various modes.

From Figure 4-8, it can be seen that the advantage in excitation that the first mode possesses over higher modes is a function of frequency. While this difference is quite large in the two middle frequencies, it is much smaller at the edges of the frequency band of interest. To highlight this effect, Figure 4-9 has been includecl. This figure displays the relative advantage of the first mode over its neighboring 


$\begin{array}{lccc}\frac{\text { Frequency }}{15.00 \mathrm{~Hz}} & \frac{3800 \mathrm{~m}}{14} & & \frac{2500 \mathrm{~m}}{7} \\ 17.75 \mathrm{~Hz} & 17 & & 9 \\ 20.00 \mathrm{~Hz} & 19 & & 10 \\ 23.50 \mathrm{~Hz} & 22 & 12 \\ 30.00 \mathrm{~Hz} & 28 & 15 \\ 35.25 \mathrm{~Hz} & 33 & 17 \\ 35.50 \mathrm{~Hz} & 34 & 18 \\ 47.00 \mathrm{~Hz} & 45 & 23 \\ 53.25 \mathrm{~Hz} & 51 & 26 \\ 55.00 \mathrm{~Hz} & 53 & 27 \\ 71.00 \mathrm{~Hz} & 68 & 35\end{array}$

Table 4-I: Number of Modes with Turning Points above $3800 \mathrm{~m}$ and $2500 \mathrm{~m}$

modes as a function of frequency. The relative advantage formula given in the figure is a crude method of quantifying how much the first mode extends alosese neighboring mode shape levels in Figure 4-8. Note the strong $30 \mathrm{~dB}$ peak at about $45 \mathrm{~Hz}$ in Figure 4-9. About $10 \mathrm{~dB}$ of the plotted value near the peak is due to the uncharacteristicly low levels of modes 2 through 5 (the modes used in the average indicated in the figure); however, a reduction of the central peak by even 10 (lB does not change the fundamental nature of the plot. The explanation of the roll off at the extremes of the frequency range is straightforward. At the lower frequencies, it is the mode shape level for the first mode that is decreasing, rather than thoie of higher modes increasing. At the high frequency end of the spectrum (above $70 \mathrm{~Hz}$ ). the mode shape levels for modes 2 and 3 finally begin to approach that of the first mode, again causing a decrease in the first mode relative advantage. Both of these effects can also be seen in Figure 4-8.

Two important conclusions must be drawn from Figures $4-8$ and $4-9$. First. the deployment of the TRISTEN source heavily favored first mode excitation. by as much as $15 \mathrm{~dB}$ or more in the middle frequencies of the range. It is not until $71 \mathrm{~Hz}$. 


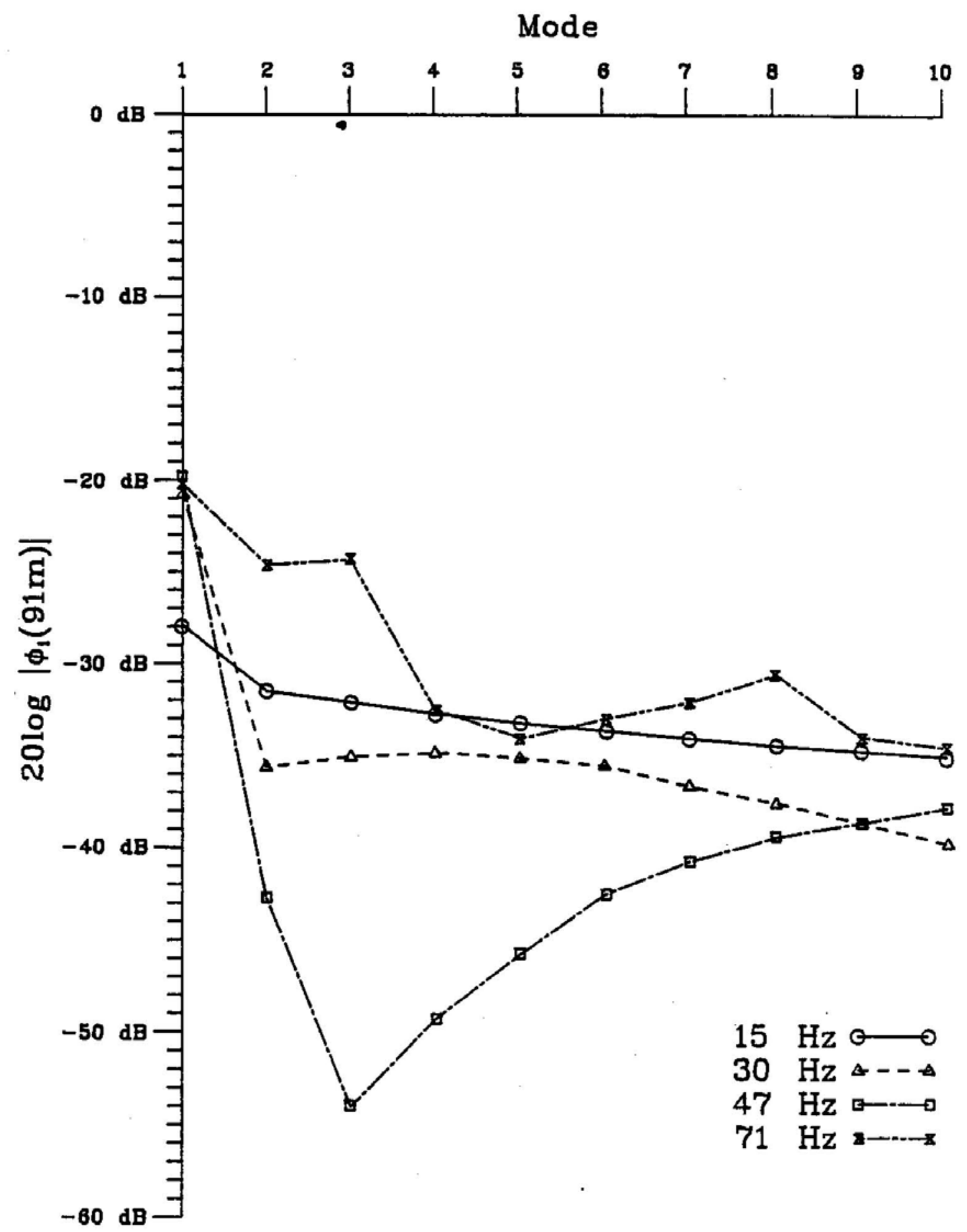

Figure 4-8: Mode Shape Levels at $91 \mathrm{~m}$ for Various Frequencies 


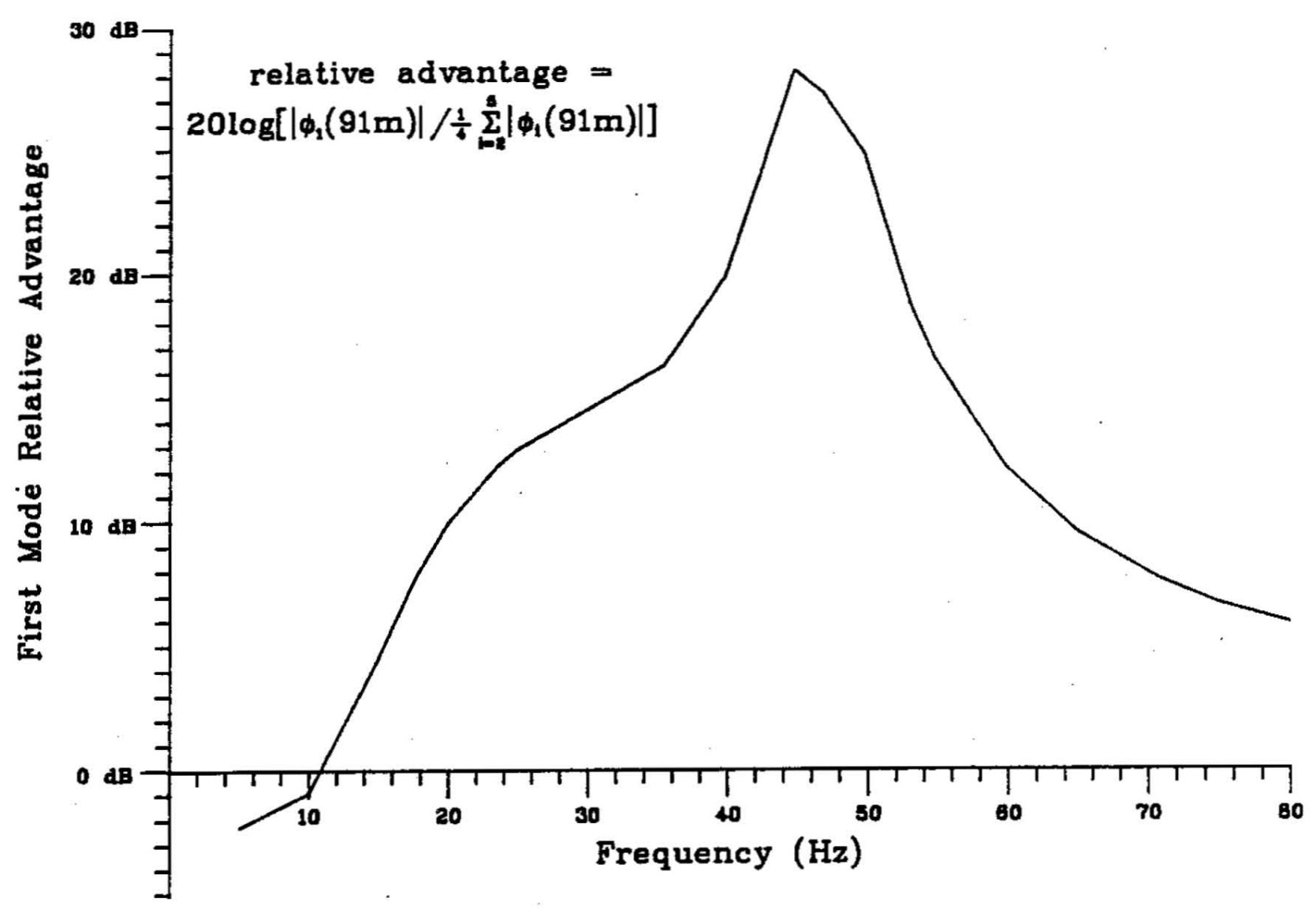

Figure 4-9: Relative Advantage of the First Mode over the Next Four Modes at $91 \mathrm{~m}$ versus Frequency

the highest frequency avalable in the vertical array data set, that the second and third modes begin to reach excitation levels equivalent with that of the first mode. Second, and equally important, because the horizontal array at FRAM was deployed to the same depth as the source, it was also heavily biased towards the first mode. so much so that data taken from the horizontal array hydrophones must be considered to be almost completely dominated by first mode characteristics. While this effect was anticipated, its magnitude is rather surprising, especially for sound propagation from the TRISTEN source to the horizontal array, where it comer into 
play at both the source and receiver.

\subsubsection{Phase Speed Analysis}

While an analysis of the mode shapes provides information about relative levels to which the source excites the various modes, an analysis of the horizontal phase speeds provides much more insight into the effects of the various parts of the sound speed profile on the modal structure. This is because the phase speeds are much more sensitive to variations in the profile than the mode shapes. Figure $t-10$ - provides plots of the phase speeds for the first three modes of the central Arctic channel in the 0 to $80 \mathrm{~Hz}$ frequency range. In general, these plots consist of three regions: a high frequency region, where the modes are effectively trapped in the surface duct; a transition region; and a low frequency region where the modal characteristics are dominated by the deeper portions of the profile. To emphasize this effect, modal phase speed results obtained from the two sound speed profiles of Figure 4-11 are also plotted. In the left hand profile, the effect of the surface duct has been eliminated by extending the profile from the $200 \mathrm{~m}$ point to the surface at the gradient existing just below that point. In the right hand profile, the surface duct has been retained and extended all the way to the bottom of the water column in a similar fashion. The gradient used here to extend the profile is the slope existing just above the $200 \mathrm{~m}$ point.

Given the results of the previous section that indicate the dominant role it plays in the transmission of sound from the TRISTEN source, a study of the first mode is of special interest here. As can be seen in Figure 4-10, below $10 \mathrm{~Hz}$ the effect of the surface duct on first mode propagation is small. From 10 to $30 \mathrm{H} z$. the mode is in transition into the surface duct, while above $30 \mathrm{~Hz}$ it can be considered to be trapped in the surface duct. As the mode falls into the surface duct, a 


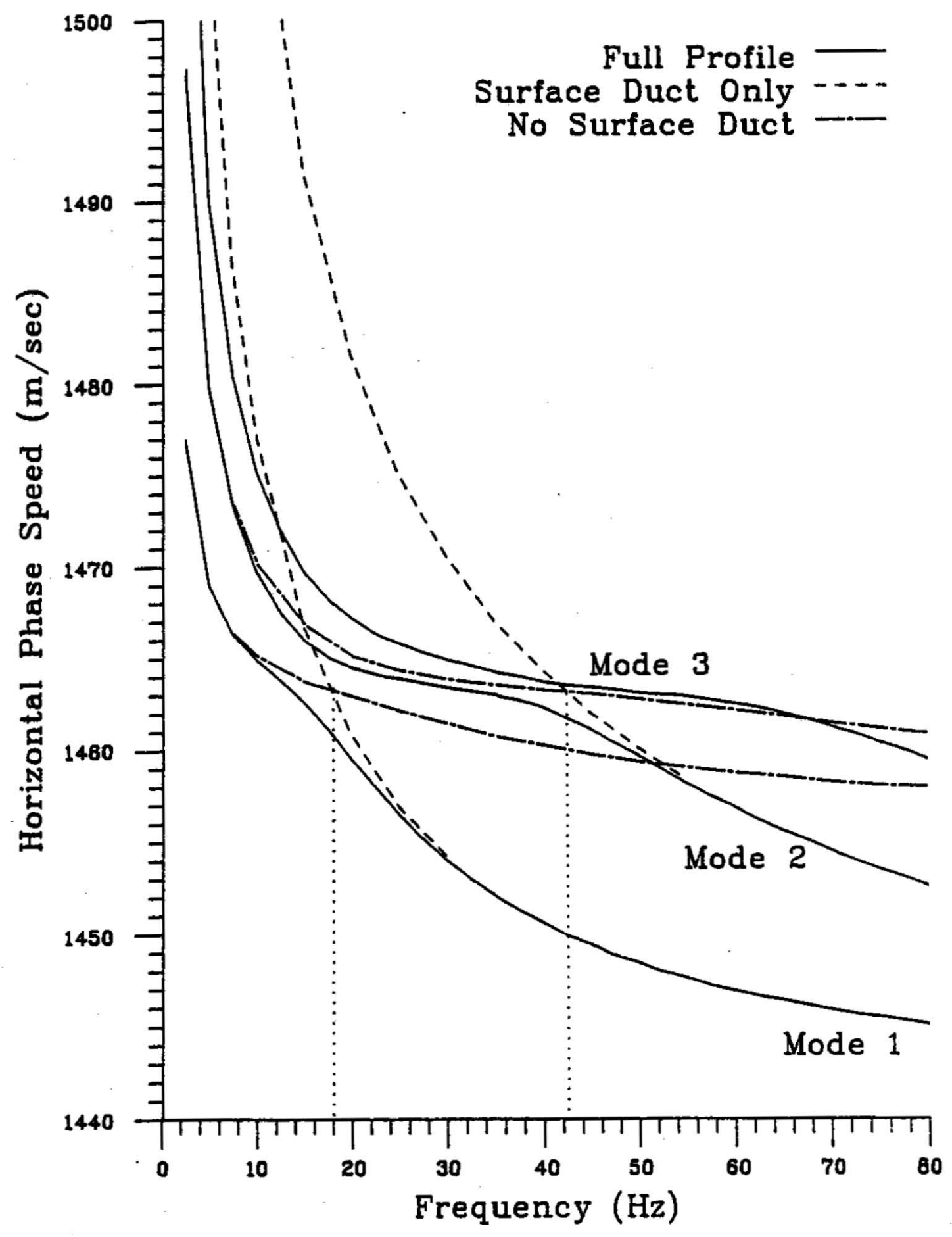

Figure 4-10: Horizontal Phase Speeds of the First Three Modes versus Frequency 

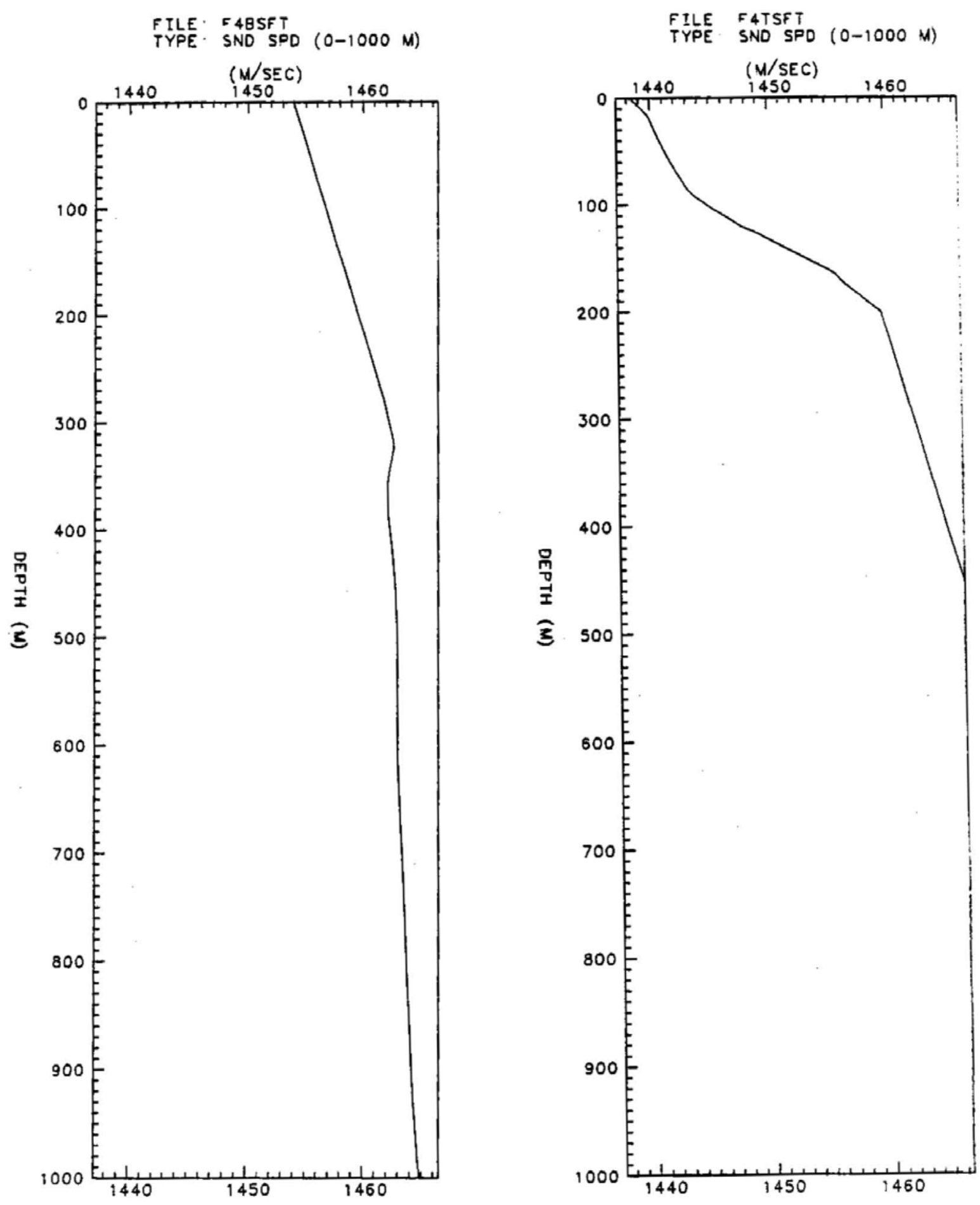

Figure 4-11: Modified Sound Speed Profiles Used as Limiting Cases for the Central Arctic Profile 
significant reduction of phase speed (from about $1460 \mathrm{~m} / \mathrm{sec}$ to about $1450 \mathrm{~m} / \mathrm{sec}$ ) is evident. Examination of the mode shape as a function of frequency (Figure 4-1) supports this conclusion. By comparison, the second mode does not begin its transition until about $35 \mathrm{~Hz}$ and is not completely trapped in the surface duct until a frequency of almost $60 \mathrm{~Hz}$ is reached. The center of the third mode transition region is about $65 \mathrm{~Hz}$, but it does not become completely trapped until well above $80 \mathrm{~Hz}$. As the various modes fall into the surface duct, the spread in horizontal phase speed from those not yet trapped becomes much more-pronounced.

Phase speed variations have also been used to investigate the sensitivity of the results to the bottom characteristics. To accomplish this, the propagating bottom of Figure 4-2 is replaced by a hard bottom and the modes recomputed at $23.5,35.25$. and $47 \mathrm{~Hz}$. Measurable differences between the resultant phase speeds and those computed earlier are first detected at modes 19,30 , and 41 , respectively. All the modes exhibiting sensitivity to the bottom structure have corresponding horizontal phase speeds in excess of $1500 \mathrm{~m} / \mathrm{sec}$, or, equivalently, possess turning points within $500 \mathrm{~m}$ of the bottom. Thus, they are excluded from the set of modes thought to most significantly contribute to propagation in the channel (those with turning points above $2500 \mathrm{~m}$ ).

\subsubsection{General Observations}

Comparing the data from Figure 4-9 with that of Figure 4-10, one observes that the frequency region in which the first mode is most dominant (between about 20 and $60 \mathrm{~Hz}$ ) coincides with it being the only mode trapped in the surface duct. It can then be concluded that any source or receiver deployed in the duct is very well tuned to the modes trapped there, but only poorly matched to those that extend outside it. In such a case, the environmental effects that most greatly affect the 
sound propagation are those that are located at the top of the water column. This is why parameters such as basement characteristics and bottom topography have relatively little impact on the problem at hand.

In the upper layer of the Arctic, the only two significant environmental considerations are the ice cover and range variations in the sound speed profile. The former is known to have a pronounced impact on the sound propagation. an mentioned earlier. At the low frequencies of interest here, the effect of the latter is probably small by comparison for a number of reasons. Surface duct range - variations influence propagation in the duct in three ways. First, they provide density and refractive index contrasts that scatter the incident sound energy, effectively increasing the attenuation. However, this effect is negligible when compared to the scattering caused by the ice, which provides a much better acoustic contrast. Second, they affect the way that the source and receiver couple into the channel by altering the shape of the modes that are trapped in the duct. Finally. they affect the distribution of modal energy by generating mode coupling. A review of the coupling coefficients (equations (2.18) and (2.19)), though, reveals that their size is primarily a function of the net change encountered in the mode shapes. Thus, both of these last two effects are controlled by the amount of mode shape variation that can be generated. In general, it can be said that the shapes of the lowest order modes (the ones which the source primarily excites) are very insensitive to small variations in the sound speed profile. These mode shapes tend to be more dependent on the larger scale trends in the profile than on its details. A change of as much as several meters per second in sound speed at any point in the duct can therefore be expected to have only a very small affect on the mode shape. Similarly, variations in duct depth of as much as 20 or $30 \mathrm{~m}$ are not overly significant. Since variations in sound speed much beyond these values are unlikely. 
it is hard to argue that range variations in the sound speed profile have an important influence on low frequency Arctic surface duct propagation.

The above conclusions are very dependent on the location of the source in depth. If the source is positioned below the surface duct, a much different situation is encountered. Under these conditions, a large spectrum of higher order modes are excited at the expense of the modes trapped in the surface duct. The botton characteristics can then be expected to play a much more important role in controlling the overall propagation picture.

TRISTEN

FRAM

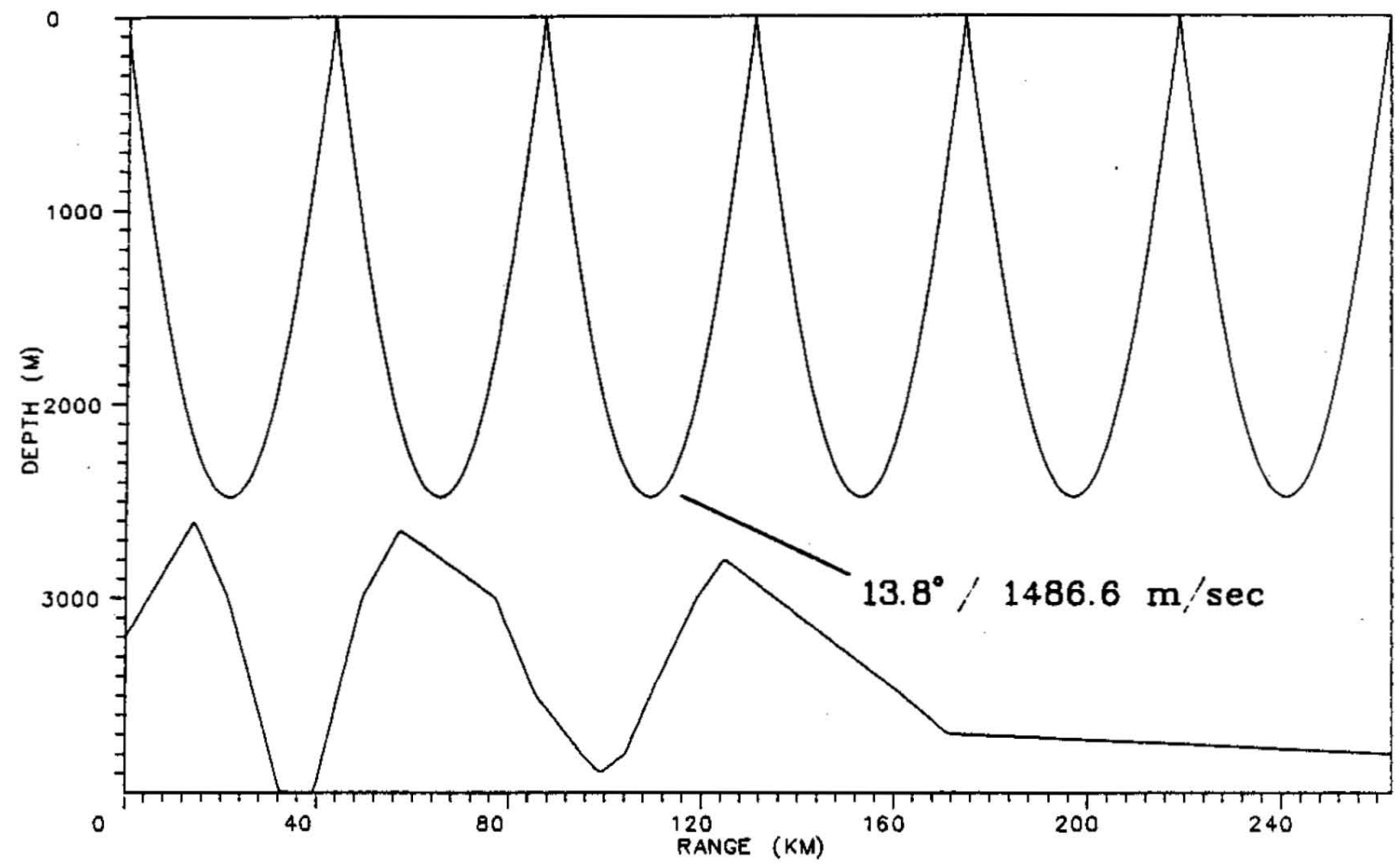

Figure 4-12: Deepest RSR Ray for the TRISTEN/FRAM Transmision Path [Note different range and depth scales.] 


\subsection{The Deepest RSR Ray Paths of the TRISTEN/FRAM Channel}

As concluded in Polcari [67], it is at times easier to characterize the propagation in terms of a ray representation than it is to describe them in terms of modes. This is especially true of the deepest diving RSR rays, which correspond to coherent sums of large numbers of higher order modes, all of which are closely spaced in horizontal phase speed. Figure 4-12 is adapted from [67] to provicle a general feel for the characteristics of the deepest RSR ray paths for the TRISTEN/FRAM transmission path. These rays are seen to exhibit horizontal phase speeds in the 1480 to $1495 \mathrm{~m} / \mathrm{sec}$ range.

\subsection{Summary}

The environmental aspects of the central Arctic sound channel and their effect on the modal structure encountered during the FRAM $N$ Experiment have been investigated. The water column sound speed profile is presented in Figure 4-1. and can be assumed to be reasonably stable over both time and space. The bottom topography creates major deterministic range dependence in the channel. The overhead ice canopy most greatly effects the attenuation of modal energy. but has little influence on the gross aspects of modal structure, the mode shapes and their horizontal phase speeds. The exact mechanism for the attenuation increase is not well understood.

The sound speed profile consists of three parts: a strong surface duct extending to about $200 \mathrm{~m}$, which is unique in that it is generated by variations in salinity rather than temperature; a monotonic increasing gradient below the surfacte duct that extends to the bottom at $3800 \mathrm{~m}$, and which is generally presillre controlled; and a basement for which some experimental data on the geophysical 
structure is available. The profile is typical of that generally encountered in the basins of the eastern region of the central Arctic. Such a profile seriously increaties. the possibility of stability problems when solving the Helmholtz equation numerically in order to obtain the mode shapes; special efforts have been made here to avoid this problem.

An examination of the mode shapes reveals that, because of the source and receiver geometries involved, first mode propagation plays a dominant role in the FRAM IV data set. It is exhibited in the relative excitation levels of the various modes by the TRISTEN source, where the first mode is often driven at levels more than $15 \mathrm{~dB}$ above those of other modes. Also, the sensitivity of the horizontal receiving array to energy propagated in the first mode exceeds its sensitivity to other modes by equal amounts. In general, the relative mode shape levels for higher order modes appear to reach a plateau whose level is not very sensitive to the frequency being considered. The shapes themselves are similar to Airy functions.

By studying variations in the associated modal horizontal phase speeds as a function of frequency, the effect of the various parts of the central Arctic sound speed profile on the modal structure has been deduced. Each mode can be seen to transition from the deeper part of the profile to the surface duct with increasing frequency; for the first mode, this transition starts at roughly $10 \mathrm{~Hz}$ and is completed by about $30 \mathrm{~Hz}$. The dominance of the first mode is intimately linked to this process. The bottom characteristics do not appear to influence the modes thought to contribute most heavily to sound propagation through the channel. Finally, the deepest propagating RSR ray paths occur at horizontal phase speeds in the 1480 to $1495 \mathrm{~m} / \mathrm{sec}$ range; these represent coherent sums of large numbers of higher order modes closely spaced in phase speed.

In general, sensors deployed in the Arctic surface duct couple very well to 
those modes that are trapped in the duct, and only poorly to any mode extending beyond it. This makes environmental effects that exist in the uppermost layer of the Arctic much more significant than effects located at other depths for any situation involving surface duct propagation. For this case, the inpact of variations in the sound speed profile appear to be small compared to that of the ice canopy. since the mode shapes of importance are relatively insensitive to small changes in the profile. Sources deployed below the duct can be expected to exhibit significantly different propagation regimes. 


\section{Chapter 5}

\section{Modal Beamforming: Theory and Simulation}

In the three previous chapters, the preliminaries necessary for "modal beamforming" have been reviewed. The source model has been discussed: the available receiving arrays have been studied in detail; and the modal structure of the sound channel has been carefully analyzed. This chapter draws upon elements of all of the three previous chapters to study the various mathematical algorithms that might be employed in the modal decomposition of a narrowband sound field at a receiving array. Such decompositions are close analogs of the methods used in traditional plane wave beamforming; from a theoretical viewpoint, the fundamental difference is the choice of the basis set on which the observed sound field is decomposed. To emphasize the similarity, the term "modal beamforming" is used here to refer to the modal decomposition process.

In plane wave beamforming, one attempts to measure the spatial structure of a narrowband signal field by estimating its three-dimensional spatial Fourier transform, or wavenumber spectrum. In modal beamforming, the estimate is of a transform that is only partially Fourier in nature; a modal transform is instead used in the vertical dimension. From a practical viewpoint, each of these choices possesses distinct advantages and disadvantages. The pure plane wave representation is very simple, yet very general, requiring only limited a priori knowledge of the characteristics of the propagation channel. This makes it a very robust, channel-independent procedure. Additionally, the extensive body of experience from other disciplines on the use (and misuse) of the Fourier transform is 
directly applicable in the study of performance issues. Finally, the existence of efficient computational algorithms for evaluation of the transform (i.e., the FFT), enhance the desirability of such an approach.

Unlike Fourier decomposition, modal beamforming requires intimate prior knowledge of the propagation channel, so that the modal structure needed to implement the algorithms can be determined. In many respects, it may be thought of as a beamforming approach that is tuned to the propagation channel in which it is implemented. As discussed in Chapter 6, modal beamforming is also less forgiving of errors in sensor position, particularly when these errors are generated by tilt in a vertical array. The added sensitivity and decreased robustness of a modal approach are balanced by a number of benefits, however. The most important of these is the very efficient representation of the received signal field provided by the modal decomposition. This efficiency has both mathematical and physical aspects. Mathematically speaking, the modal representation provides a countably infinite basis set as opposed to the infinitely dense set of plane waves; further, this modal basis set can often be limited to a small number of significantly contributing modes with relatively simple arguments (such as those presented in Chapter 2). From a physical viewpoint, the modal approach is in some sense the natural decomposition to employ, because it represents a direct eigenfunction representation of the sound field.

The chapter starts with the derivation of modal beamformers in a general sense from the principles of least squares estimation theory. The modal equivalents of two popular plane wave beamforming techniques are developed: spatial matched filtering; and maximum liklihood (MLM) algorithm. Each of these methods can be implemented in either a single beam or a multiple beam variant, providing a total of four different approachs. A comparison of the four is made to highlight their 
theoretical differences. Two issues then remain. The first is the question of relative performance, which, because of its complexity, is studied in some detail. The other, which is closely related, involves the proper selection of a modal source model. Both of these issues are investigated using simulated data in addition to theoretical results.

The theoretical developments presented here are covered in the literature to varying degrees. The fundamental techniques of least squares estimation theory have a rich history, and any number of good standard references are available: one such example is Lawson and Hanson [49]. References which deal with direct modal amplitude estimation techniques are limited. Hinich [42] first proposed the multiple beam MLM algorithm derived here, but did not applied it to actual data. Shang. Clay, and Wang [75] develop the multiple beam least squares algorithm and apply it to data generated in a laboratory setting. Signal processing aspects of the algorithm (particularly performance issues) are not discussed, however. While Ferris [3:3] uses a single beam least squares algorithm to make modal amplitude estimates from field data, his experiment is purposely structured to avoid the full modal decomposition issue. A similar approach is taken true of the tow tank experiment of Hobalek. Tindle, and Muir [44].

More research has been completed in two related areas. One of these areas is the study of the plane wave equivalents to the algorithms investigated here. Baggeroer [2] provides a comprehensive reference on plane wave beamforming, including the MLM approach, which was originally introduced in the classic paper by Capon [10]. Cox [21] analyzes the performance of MLM plane wave beamformers in detail. Schweppe [ $\boldsymbol{\pi} 4]$ studies the concept of multiple beam least squares beamforming. Finally, a recent paper by Hayes, Ripa, and Mangum [38] provides further insight into the relationship between the least squares and 
MLM approaches.

Related work, much of it recent, can also be found in the area that has come to be known as "matched field processing". The techniques of interest in this field are not directly involved with modal decomposition; rather, they use prior predictions of the total signal field to generate direct estimates of source parameters, such as range and depth. However, their performance is fundamentally linked to the modal characteristics of the sound channel in which they are employed, making them of much interest in the present effort. Clay [17] first proposed the use of matched field processing; this work also forms the basis for Chapter 7 of Tolstoy and Clay [79] and Appendix A5 of Clay and Medwin [18]. In a more recent paper, Bucker [9] proposes a similar but more robust scheme. Hinich develops an MLM algorithm for source depth estimation [42] and studies the problem of the optimal array locations for making such estimates [43]. Heitmeyer. Moseley, and Fizell [39] have completed detailed simulations of matched field processing in a Pekeris waveguide, while Porter, Dicus, and Fizell [70] have done similar work for a deep Pacific channel. Fizell and Wales [34] have had some preliminary success applying the technique to actual field data from the Arctic Ocean. Finally, Shang, Clay, and Wang [75] have proposed a related (but somewhat more simplistic) approach for passive source ranging.

\subsection{Development of the Least Squares Modal Beamformer}

The theoretical development of the least squares modal beamformer is straightforward. Let the $N \times 1$ complex vector $\underline{\mathbf{P}}$ denote the complex amplitude observed at each of the $N$ sensors in the array after completion of the two steps of quadrature demodulation and filtering described in Section 3.11. For the moment, assume that $\dot{\mathbf{P}}$ is a constant; the case of temporally varying complex amplitudes is 
taken up in the sequel. With the exception of the assumption of time invariance, $\underline{\mathbf{P}}$ is then identical to $\mathbf{Q}(t)$ in equation (1.5). Because $\underline{\mathbf{P}}$ is a complex vector. its elements include information about both the magnitude and phase of the signal.

The least squares beamformer computes the set of complex modal amplitudes which minimizes the total square error between the received pressure field and the modal source description developed in Chapter 2. This error may be quantified by defining an error vector

$$
\underline{\mathbf{e}}=\underline{\mathbf{P}}-\underline{\overline{\mathbf{E}}} \underline{\hat{\mathbf{A}}} .
$$

The second term in this equation represents the assumed source model summarized in equations (2.33) through (2.36). $\underline{\hat{\mathbf{A}}}$ is an $M \times 1$ time invariant complex column vector representing the resulting magnitude and phase estimates for the amplitude of the $M$ modes that are being modeled. Note that the choice of $M$ is a modeling issue of some complexity whose resolution is left to the user. The resultant performance of the processing is quite sensitive to the proper selection of this parameter, as investigated in Section 5.10. The $N \times M$ steering matrix $\underline{\bar{E}}$ is the same as that described in equation (1.4).

The least squares requirement is that the real scalar

$$
\begin{aligned}
Q & =\underline{\mathbf{e}}^{+} \underline{\mathbf{e}} \\
& =\underline{\mathbf{P}}^{+} \underline{\mathbf{P}}-\underline{\mathbf{P}}^{+} \underline{\overline{\mathbf{E}}} \underline{\hat{\mathbf{A}}}-\underline{\hat{\mathbf{A}}}^{+} \underline{\overline{\mathbf{E}}}^{+} \underline{\mathbf{P}}+\underline{\hat{\mathbf{A}}}^{+} \underline{\overline{\mathbf{E}}}^{+} \underline{\overline{\mathbf{E}}} \underline{\hat{\mathbf{A}}}
\end{aligned}
$$

be minimized over all possible choices of the $\underline{\hat{\mathbf{A}}}$ vector. The minimization is easily performed by variational calculus. Setting the first variation of equation (5.2) to zero, one obtains 


$$
\begin{aligned}
\partial Q & =0 \\
& =\left(\underline{\hat{\mathbf{A}}}^{+} \underline{\overline{\mathbf{E}}}^{+} \underline{\overline{\mathbf{E}}}-\underline{\mathbf{P}}^{+} \underline{\overline{\mathbf{E}}}\right) \partial \underline{\hat{\mathbf{A}}}+\partial \underline{\hat{\mathbf{A}}}^{+}\left(\underline{\overline{\mathbf{E}}}^{+} \underline{\overline{\mathbf{E}}} \underline{\hat{\mathbf{A}}}-\underline{\overline{\mathbf{E}}}^{+} \underline{\mathbf{P}}\right),
\end{aligned}
$$

which requires that

$$
\underline{\overline{\mathbf{E}}}^{+} \underline{\overline{\mathbf{E}}} \underline{\hat{\mathbf{A}}}=\overline{\mathbf{E}}^{+} \underline{\mathbf{P}} \text {. }
$$

Assuming the $M \times M$ matrix $\underline{\overline{\mathbf{E}}}^{+} \underline{\overline{\mathbf{E}}}$ to be non-singular, equation (5.4) may be solved directly to find the least squares modal estimator

$$
\underline{\hat{\mathbf{A}}}=\left(\underline{\overline{\mathbf{E}}}^{+} \underline{\overline{\mathbf{E}}}\right)^{-1} \underline{\overline{\mathbf{E}}}^{+} \underline{\mathbf{P}} \text {. }
$$

Such an assumption requires that $M$ not be larger than $N$. This requirement may be circumvented by identifying the linear transformation in equation (5.5) as the standard matrix pseudo-inverse; the techniques for extending this operator to the case where $M$ is larger than $N$ are quite standard [49]. Such an approach has a number of drawbacks, though; the most important being that the resulting modal amplitude estimates are no longer linearly independent. Therefore, it is not considered here. From a practical standpoint, the assumption of non-singularity also raises the issue of the numerical precision needed to accurately compute the inverse, a question intimately linked to the proper selection of $M$.

An investigation of the second variation demonstrates that the solution results in a minimum total square error if the $\underline{\bar{E}}^{+} \underline{\underline{E}}$ matrix is positive definite (which it must be if it is non-singular),

$$
\partial^{2} Q=2 \partial \underline{\hat{\mathbf{A}}}^{+} \underline{\overline{\mathbf{E}}}^{+} \underline{\overline{\mathbf{E}}} \partial \underline{\hat{\mathbf{A}}}>0 .
$$

The residual total square error for the beamformer can be obtained by substituting equation (5.5) back into equation (5.2) and simplifying, which results in 


$$
Q_{\min }=\underline{\mathbf{P}}^{+}\left[\overline{\mathbf{I}}-\underline{\overline{\mathbf{E}}}\left(\overline{\mathbf{E}}^{+} \underline{\overline{\mathbf{E}}}\right)^{-1} \overline{\mathbf{E}}^{+}\right] \underline{\mathbf{P}} \text {. }
$$

Based on equation (1.6), an estimate of the $M \times M$ modal cross-coherence matrix may be computed as

$$
\begin{aligned}
\underline{\hat{\mathbf{S}}}_{\mathrm{M}} & =\mathrm{E}\left[\underline{\hat{\mathbf{A}}} \underline{\hat{\mathbf{A}}}^{+}\right] \\
& =\left(\underline{\overline{\mathbf{E}}}^{+} \overline{\overline{\mathbf{E}}}\right)^{-1} \underline{\overline{\mathbf{E}}}^{+} \underline{\overline{\mathbf{S}}}_{\mathrm{N}} \overline{\mathbf{E}}\left(\underline{\overline{\mathbf{E}}}^{+} \underline{\overline{\mathbf{E}}}\right)^{-1},
\end{aligned}
$$

where

$$
\overline{\mathbf{s}}_{\mathrm{N}}=\mathrm{E}\left[\underline{\mathbf{P}} \underline{\mathbf{P}}^{+}\right]
$$

is the more traditional $N \times N$ sensor cross-coherence matrix.

If the vector $\underline{\boldsymbol{P}}$ is actually time invariant, then the modal cross-coherence estimator is redundant, since it provides no more information than the amplitude estimate given in equation (5.5). On a practical basis, though, this vector fluctuates randomly over time. The application of equation (5.5) to such a problem can be justified only on an instantaneous basis. One can envision using a time slice of data to form $\underline{\mathbf{P}}$, which is then transformed into the complex modal amplitude estimates for that instant of time. Under such conditions, equation (5.5) provides only an estimate of the random complex amplitude time series of each mode, since it includes no averaging. By contrast, equation (5.8) yields information that is both more useful and more stable, since it instead estimates the second order spatial statistics of the vector random process. Since the expected value operator is almost always implemented by temporal averaging rather than ensemble averaging, the modal cross-coherence estimator may be thought of as averaging a number of estimates of the matrix $\underline{\hat{\mathbf{A}}} \underline{\hat{A}}^{+}$, where $\underline{\hat{\mathbf{A}}}$ is determined on an instantaneous basis 
from equation (5.5). Alternatively, one can view the averaging taking place in the computation of the sensor cross-coherence matrix, with equation (5.8) providing the transformation to the equivalent modal cross-coherence matrix. The two views are identical because the processor is linear.

\subsection{Single Beam versus Multiple Beam Beamformers}

As developed, equations (5.5), (5.7), and (5.8) define the multiple beam least squares modal beamformer. This beamformer is exactly analogous to Schweppe's multiple beam decoupled processor [74] for plane wave beamforming, with the sole exception that the construction of steering matrix $\underline{\overline{\mathbf{E}}}$ is different. While it is possible to implement multiple beam beamformers directly, a different approach is more commonly used, especially in azimuthal plane wave beamforming. A single plane wave source model is chosen, so that $M=1$. The resulting algorithm is then evaluated repeatedly for various steering vectors, each of which corresponds to a plane wave propagating in a different direction. One can develop an equivalent approach for modal beamforming by again selecting $M$ to be 1 , and then making successive independent estimates by indexing the steering vector over the mode set of interest. This implementation is called the single beam least squares beamformer in this thesis.

Such an approach has several advantages. Most importantly, it eliminates the need for the user to select a value for $M$ a priori. The resulting processor is simple and intuitive, forming the modal equivalent of a conventional beamformer. Alternatively, it may be considered to be a spatial implementation of the matched filter concept. Finally, the requirement that $M$ be less than $N$ is relased, so that one is not constrained in the number of different modes that can be estimated.

As long as the mode set of interest is smaller than the number of selnors 
available in the array, the processing can be implemented by either approach. Therefore, it is important to understand the relationship of the two methods. This can be readily demonstrated with a simple example. Consider a signal which consists only of a single mode, with no background noise. Let the steering vector associated with the mode forming the signal be denoted by $\underline{\mathbf{E}}_{p}$, and let $a_{p}$ be the mode's complex amplitude. In this situation, the sensor cross-coherence matrix is

$$
\overline{\mathbf{S}}_{\mathrm{N}}=\left|a_{p}\right|^{2} \underline{\mathbf{E}}_{p} \underline{\mathbf{E}}_{p}^{+} \text {. }
$$

Assume that the single beam least squares beamformer is steered to a mode different than the one present in the signal. Let the steering vector associated with this mode be $\underline{\mathbf{E}}_{b}$. From equation (5.8), the resulting single beam least squares estimate for the squared magnitude of this mode is easily found to be

$$
\left|\hat{a}_{b}\right|^{2}=\left|a_{p}\right|^{2} \frac{\left|\underline{\mathbf{E}}_{b}^{+} \underline{\mathbf{E}}_{p}\right|^{2}}{\left|\underline{\mathbf{E}}_{p}\right|^{4}}
$$

Note that when the beamformer is steered to the mode actually present in the signal, the estimate in equation (5.11) is exact. When steered to any other mode, though, what is generally a small amount of energy (but need not necessarily be) is allowed to leak through, contaminating the estimate to a greater or lesser degree. Such behavior is equivalent to the sidelobe patterns developed in plane wave beamforming.

Now consider a multiple beam least squares beamformer with $M=2$. Let one of the two modes included in the beamformer be the mode actually present in the signal; let the other be the same mode to which the single beam beamformer is steered. The steering matrix for the multiple beam algorithm is then

$$
\overline{\mathbf{E}}=\left[\underline{\mathbf{E}}_{p} \mid \underline{\mathbf{E}}_{b}\right]
$$


While algebraicly tedious, it is not difficult to directly evaluate equation (5.8) for the sensor cross-coherence matrix of equation (5.10) and the steering matrix given in equation (5.12). An estimate equivalent to that of equation (5.11) may be extracted from the lower right hand corner element of $\hat{\bar{S}}_{\mathbf{M}^{*}}$. When this is done, it is found that

$$
\left|\hat{a}_{b}\right|^{2}=0 \text {. }
$$

The relationship between the single beam and multiple beam least squares beamformers is now evident. The single beam algorithm allows energy from one mode to leak into the estimates of other modes, while the multiple beam algorithm does not allow such leakage between modes that are included in the beamformer. The effect of the multiple beam approach is best described by saying that the effective beam pattern for each mode to be estimated is forced to have a null in the direction of each of the other modes also included in the beamformer. This elimination of cross-talk and sidelobe leakage between the various modal estimates represents the fundamental advantage of multiple beam algorithms over their single beam equivalents.

\subsection{Generalizations of the Least Squares Modal Beamformer and the MLM Modal Beamformer}

The results of Section 5.1 can be generalized through the introduction of a weighting matrix $\underline{\mathbf{W}}$ in the error calculation of equation (5.2). $\overline{\mathbf{W}}$ is $N \times N$, and is assumed to be Hermetian and positive definite. It controls the relative importance of the various terms that contribute to the error expression. The generalized form of equation (5.2) is 


$$
\begin{aligned}
& Q=\underline{\mathbf{e}}^{+} \underline{\overline{\mathbf{W}}} \underline{\mathbf{e}} \\
& =\underline{\mathbf{P}}^{+} \underline{\overline{\mathbf{W}}} \underline{\mathbf{P}}-\underline{\mathbf{P}}^{+} \underline{\mathbf{W}} \underline{\mathbf{E}} \underline{\hat{\mathbf{A}}}-\underline{\hat{\mathbf{A}}}^{+} \underline{\overline{\mathbf{E}}}^{+} \underline{\overline{\mathbf{W}}} \underline{\mathbf{P}}+\underline{\hat{\mathbf{A}}}^{+} \underline{\overline{\mathbf{E}}}^{+} \underline{\overline{\mathbf{W}}} \underline{\mathbf{E}} \underline{\hat{\mathbf{A}}} .
\end{aligned}
$$

for which the minimum total square error is generated by

$$
\underline{\hat{\mathbf{A}}}=\left(\underline{\overline{\mathbf{E}}}^{+} \underline{\overline{\mathbf{W}}} \underline{\overline{\mathbf{E}}}\right)^{-1} \underline{\overline{\mathbf{E}}}^{+} \underline{\overline{\mathbf{W}}} \underline{\mathbf{P}} \text {. }
$$

The residual error result equivalent to equation (5.7) is

$$
Q_{\min }=\underline{\mathbf{P}}^{+}\left[\underline{\overline{\mathbf{W}}}-\underline{\overline{\mathbf{W}}} \underline{\overline{\mathbf{E}}}\left(\underline{\overline{\mathbf{E}}}^{+} \underline{\overline{\mathbf{W}}} \underline{\overline{\mathbf{E}}}\right)^{-1} \underline{\mathbf{E}}^{+} \overline{\overline{\mathbf{W}}}\right] \underline{\mathbf{P}} \text {, }
$$

and the modal cross-coherence matrix estimator becomes

$$
\underline{\hat{\mathbf{S}}}_{M}=\left(\underline{\bar{E}}^{+} \underline{\overline{\mathbf{W}}} \underline{\overline{\mathbf{E}}}\right)^{-1} \underline{\underline{\mathbf{E}}}^{+} \overline{\underline{\mathbf{S}}}_{\mathrm{N}} \overline{\overline{\mathbf{E}}}\left(\overline{\underline{\mathbf{E}}}^{+} \underline{\overline{\mathbf{W}}} \overline{\underline{\mathbf{E}}}\right)^{-1} \text {. }
$$

The choice of $\underline{\bar{W}}$ is somewhat arbitrary. The results reduce to those of the least squares beamformer if $\underline{\bar{W}}$ is chosen to be an $N \times N$ identity matrix. Other choices are possible; the discussion here is limited to the selection often made in high resolution beamforming

$$
\overline{\mathbf{W}}=\overline{\mathbf{s}}_{\mathrm{N}}^{-1} \text {. }
$$

The motivation behind choosing the inverse of the sensor cross-coherence matrix to be the weighting matrix is quite simple, but the implementation of such a choice is rather complex. In theory, one desires to weight observations made on sensors having low noise more heavily than those encountering high noise levels. Obviously, if the noise is uncorrelated between sensors and has the same power (variance) at all the hydrophones, then selecting $\overline{\mathbf{W}}$ to be the identity matrix is 
reasonable. For spatially uncorrelated noise that is of varying intensity at different sensors, it is appropriate to choose $\underline{\overline{\mathbf{W}}}$ as a diagonal matrix with each non-zero element being proportional to the inverse of the noise power seen at the respective sensor. Such a selection most certainly weights the sensors receiving low noise lev els more heavily than those receiving high noise levels.

For spatially coherent noise, the concept is naturally extended by identifying the weighting matrix with the inverse of the full sensor cross-coherence matrix in a condition where no signal is present, so that only noise statistics are measured. Finally, it is shown in Appendix A that the results of equations (5.15) and (5.17) are invariant to the inclusion of signal components in the weighting matrix, so that the full sensor cross-coherence matrix may be used in place of just its noise portion. This invariance is very important, since it allows in situ adaptive estimates of the weighting matrix to be made without requiring prior removal of the signal from the noise. To be valid, it does require the assumption of statistical independence between signal and noise, though, which then leads to the issue of coherent interference. This very significant problem is addressed at length in the sequel.

The choice of $\underline{\bar{W}}$ indicated in equation (5.18) allows equations (5.15) and (5.17) to be recast in more recognizable forms. After simplification, one obtains

$$
\underline{\hat{\mathbf{A}}}=\left(\underline{\overline{\mathbf{E}}}^{+} \underline{\overline{\mathbf{S}}}_{\mathrm{N}}^{-1} \overline{\mathbf{E}}\right)^{-1} \underline{\overline{\mathbf{E}}}^{+} \overline{\underline{\mathbf{S}}}_{\mathrm{N}}^{-1} \underline{\mathbf{P}},
$$

and

$$
\underline{\overline{\mathbf{S}}}_{\mathrm{M}}=\left(\underline{\overline{\mathbf{E}}}^{+} \overline{\overline{\mathbf{s}}}_{\mathrm{N}}^{-1} \underline{\overline{\mathbf{E}}}\right)^{-1}
$$

These results are the modal analog of what is known as the MLM algorithm: the name minimum energy method is also commonly used. Equations $(5.19)$ and $(5.20)$ may be derived in a number of different ways, each of which provides insight into 
the properties of the solution. The name "MLM algorithm" comes from the fact that the solution is the maximum liklihood estimator for a known signal in zero mean, spatially coherent Gaussian noise. It can also be shown that the solution provides the maximum SNR processor for a signal in statistically independent coherent noise.

The MLM algorithm just derived represents the modal analog of the full multiple beam MLM beamformer. It agrees with the result of Hinich [42], who approached the problem from the perspective of a maximum liklihood estimator for a signal in Gaussian noise. The algorithm can also be implemented as an analog of the single beam least squares approach, where $M$ is chosen as 1 and the resulting processor is repeatedly evaluated by indexing the steering vector $\mathbf{E}$ over the mode set of interest. The relationship between the single beam and multiple beam variants of the MLM algorithm is identical to that of the two least squares approaches, in that the effective beam pattern for each mode included in the multiple beam algorithm is forced to have nulls in the direction of all the other modes also included; such nulls are not generated in the single beam ML.II algorithm. This null generation has some interesting consequences for ML.M algorithm performance. These effects are discussed later in the chapter.

\subsection{A Comparison of Modal Beamforming with Plane Wave Beamforming}

As is apparent from the previous discussion, the most significant difference between a modal beamforming implementation and more traditional plane wave methods is in the construction of the steering matrix $\underline{\underline{\mathbf{E}}}$. If one considers the single beam case for simplicity, the steering vector required for the detection of a plane wave traveling with wavenumber vector $\vec{\kappa}$ is 


$$
\underline{E}=\left[\begin{array}{c}
e^{-j \vec{k} \cdot \vec{r}_{1}} \\
e^{-j \vec{k} \cdot \vec{r}_{2}} \\
\cdot \\
e^{-j \vec{k} \cdot \vec{r}_{N}}
\end{array}\right]
$$

where $\vec{r}_{i}$ is the three-dimensional position vector of the $i^{\text {th }}$ sensor with respect to the receiver reference point. For the plane wave case, $\vec{\kappa}$ has a magnitude equal to the wavenumber, and a direction that is parallel to the direction of propagation. It also is three-dimensional, so that it may include a component in $z$. Because a plane wave is homogeneous in amplitude across any phase front, the terms of the steering vector all have unit magnitude. The phase of each term indicates the compensation necessary to correct for the phase accumulation of the wave in its travel from the receiver reference point to the sensor.

By contrast, the steering vector describing the $m^{\text {th }}$ mode is

$$
\underline{\mathbf{E}}=\left[\begin{array}{c}
\phi_{m}\left(z_{1}\right) e^{-j \vec{\kappa}_{m} \cdot \vec{r}_{1}} \\
\phi_{m}\left(z_{2}\right) e^{-j \vec{\kappa}_{m} \cdot \vec{r}_{2}} \\
\dot{\cdot} \\
\phi_{m}\left(z_{N}\right) e^{-j \vec{\kappa}_{m} \cdot \vec{r}_{N}}
\end{array}\right],
$$

where $\phi_{m}(z)$ denotes the shape of the mode, and $\vec{\kappa}_{m}$ is the horizontal wavenumber vector associated with the mode. $\vec{\kappa}_{m}$ has a length equal to the mode's horizontal wavenumber and a direction parallel to the horizontal direction of propagation. In contrast to the plane wave case, both the wavenumber vector $\vec{\kappa}_{m}$ and the position vectors $\vec{r}_{i}$ are now restricted to just the two horizontal dimensions. Also, the modal steering vector elements are non-uniformly weighted by the size of the mode shape at each sensor's depth. These two differences are indicative of the fact that the transform used in the $z$ direction is modal rather than Fourier.

It is of interest to specialize these results to the case of a horizontal array. For 
such a situation, the depths of all the sensors can be assumed equal. This makes all the $\phi_{m}\left(z_{i}\right)$ in the modal steering vector identical. For the plane wave steering vector, it also ensures that the phase contribution of the $z$ component of the wavenumber vector is constant across all the array elements. For a horizontal array, therefore, each modal steering vector can be considered to be related to an equivalent plane wave steering vector. The only differences between these two steering vectors are a scalar phase factor and a scalar magnitude factor. One can easily compensate for the effect of the magnitude scalar, since it is equal to the size of the mode shape at the horizontal array deployment depth, while the phase scalar has no effect on the modal cross-coherence estimate, which provides only relative phase information. Thus, for a horizontal array, plane wave beamforming in the vertical direction provides information which is equivalent to the modal amplitude estimates of direct modal beamformers. This observation becomes important in Chapter 7, where data from the horizontal array (which is processed with plane wave beamformers) is compared with direct modal beamforming results from the vertical array.

\subsection{Estimation of the Sensor Cross Coherence Matrix}

The beamformers used for the generation of the data presented in this thesis are fashioned around estimates of the modal cross-coherence matrix (equations (j., $)$ and (5.20)) rather than the estimates of the direct complex modal amplitudes (equations (5.5) and (5.19)). There are several reasons for selecting this approach. As discussed in Section 5.1, the cross-coherence matrix is related to the fundamental statistics of the random amplitude processes, whereas direct amplitude measurements provide data only about a single realization of these processes. Thus. the former can be considered to be somewhat more stable than the latter. 
Additionally, the cross-coherence matrix estimator is slightly more efficient computationally, because its implicit averaging reduces the number of data points that must be processed through the beamformer. Finally, the matrix estimate provides direct measurements of the coherence for the various modes in its offdiagonal terms. The only information lost in this approach is the absolute phase of the received signal, which is of no interest here.

As written, equations (5.8) and (5.20) depend upon the sensor cross-coherence matrix $\overline{\mathbf{S}}_{\mathrm{N}}$, which is an unknown statistic of the random propagation problem. In practice, $\overline{\mathbf{S}}_{\mathrm{N}}$ is first estimated over a finite window of data, and then this estimate is substituted into the equations in place of the true sensor cross-coherence matrix. Typically, $\underline{\hat{\mathbf{S}}}_{\mathrm{N}}$ would be computed by integrating over the band of frequencies containing the signal. For discrete time signals that have been complex demodulated, the calculation may be written as [10]

$$
\underline{\overline{\mathbf{S}}}_{\mathrm{N} i k}=\sum_{m=-B}^{B} X_{i}(m) X_{k}^{*}(m),
$$

where $2 B+1$ is the bandwidth of the signal and $X_{i}(m)$ is the FFT of $x_{i}(n)$, the complex preprocessed time series received at the $i^{\text {th }}$ sensor.

A somewhat simpler procedure may be used in the present situation. Becaluse of its narrowband nature, the signal may be assumed to reside in only a single frequency bin. The preprocessed time series from each sensor may then be viewed as successive estimates of that bin's complex amplitude, since the digital filtering employed is very narrow and cuts off quite sharply. For these conditions, a natural estimate of the sensor cross-coherence matrix is the direct time average

$$
\stackrel{\overline{\mathrm{S}}}{\mathrm{N} i k}_{L}=\frac{1}{L} \sum_{n=0}^{L} x_{i}(n) x_{k}^{*}(n) .
$$


This method has the advantage of not requiring FFT computations in the estimation procedure. The two approaches are easily shown to be equivalent. If the sum in equation (5.23) is extended to cover all frequency bins generated by the FFT, then the results of equations (5.23) and (5.24) must be equal by Parseval's relation. Since the narrowband nature of the preprocessing ensures that any energy remaining in frequency bins outside of the $12.5 \mathrm{mHz}$ analysis bandwidth is negligible, the resulting estimates must then be the same.

The replacement of $\overline{\mathbf{S}}_{\mathrm{N}}$ by an estimate raises the issue of bias generation in the estimates of $\overline{\mathbf{S}}_{\mathbf{M}}$. This is not a problem with the least squares algorithns, since only linear transformations are made on the unbiased estimate of the sensor crosscoherence matrix. Such is not the case for the MLM algorithms, though, because they involve non-linear inversion operations. Both Capon and Goodman [11] and Scheer [73] have investigated the resulting bias in some detail. While this is generally a matter of some concern when using MLM algorithms, the coherent interference problem (to be discussed presently) causes such large errors in this application that the bias issue may be considered moot here. None the less. it should be noted that, in general, absclute levels returned by MLM algorithms must be corrected in order to guarantee quantitative validity.

\subsection{Performance of the Various Modal Beamformers}

The previous discussion defines four different possible approaches to the modal beamforming problem. These approaches are:

1. Single beam least squares (conventional) modal beamforming.

2. Multiple beam least squares modal beamforming.

3. Single beam MLM modal beamforming. 
4. Multiple beam MLM modal beamforming.

Judicious selection from among these various methods requires that their relative performances be assessed under conditions likely to be encountered during the actual data reduction. The next several sections address this task. The length and complexity of the discussion make a short overview of the eventual conclusions in order.

The theoretical performance of the single beam least squares beamformer is investigated in next section. This simple approach has two attractions: it is easy to implement; and it is quite robust. However, even fundamental theoretical calculations demonstrate that the FRAM IV vertical array allows only the first mode to be reliably estimated at the lowest frequencies of interest. Such performance is clearly inadequate, so that the other algorithms must then be considered.

The two MLM algorithms are investigated in the following section. Mode coherence is shown to be a major factor in the performance assessment of both these methods. The single beam algorithm is shown to be theoretically superior to the multiple beam algorithm if the individual acoustic modes that make up the signal are incoherent (phase random) with respect to each other. This method would be the processor of choice for such a situation, since it also offers higher resolution than either of the least squares approaches. One of the most significant conclusions of Chapter 7 is that the received mode field is highly coherent in the Arctic, though. Indeed, some preliminary indications of this conclusion have already been presented in Figures 3-9 through 3-12. For a signal field consisting of coherent modes, both the single and multiple beam MLM algorithms perform inadequately when applied to the vertical array data, although they may still be successfully applied to the horizontal array data. This issue is discussed in some length, and it is demonstrated 
that the fundamental cause of the difficulties is mutual coherent interference between the various modes.

In the final section, the multiple beam least squares beamformer is studied. This approach is shown to possess somewhat better resolution than the single beam least squares processor and is not affected by the coherent interference issues that plague the two MLM algorithms. Therefore, the multiple beam least squares method is selected for use in reducing the vertical array data.

\subsection{Performance of the Single Beam Least Squares Beamformer}

The fundamental issue involved in any assessment of the performance of the single beam least squares beamformer is its ability (or inability) to distinguish between signal energy propagating in different modes. To investigate this question. Figure 5-1 has been generated to provide a measure of the theoretical modal resolution that can be expected from the algorithm. This figure consists of a contour plot of a surface, the height of which represents the output of the beamformer for an array consisting of the 18 recorded elements of the FRAMI $\Gamma^{\circ}$ vertical array. The vertical axis shows the mode which the beamformer is to detect (or, in a more traditional parlance, the mode to which the beamformer is steered), while the horizontal axis indicates the one mode actually present in the artificially created signal field which the beamformer is processing. The theoretical output of the beamformer for each possible combination of the mode actually present and the mode steered to is used to define the surface, which is then contoured on a dB scale (2.5 $\mathrm{dB}$ per contour). The first 10 modes of the $30 \mathrm{~Hz}$ mode set have been used in the generation of Figure 5-1.

In analogy to plane wave beamforming in bearing, each horizontal crosssection of Figure 5-1 may be considered to be the modal "beam pattern" that is 

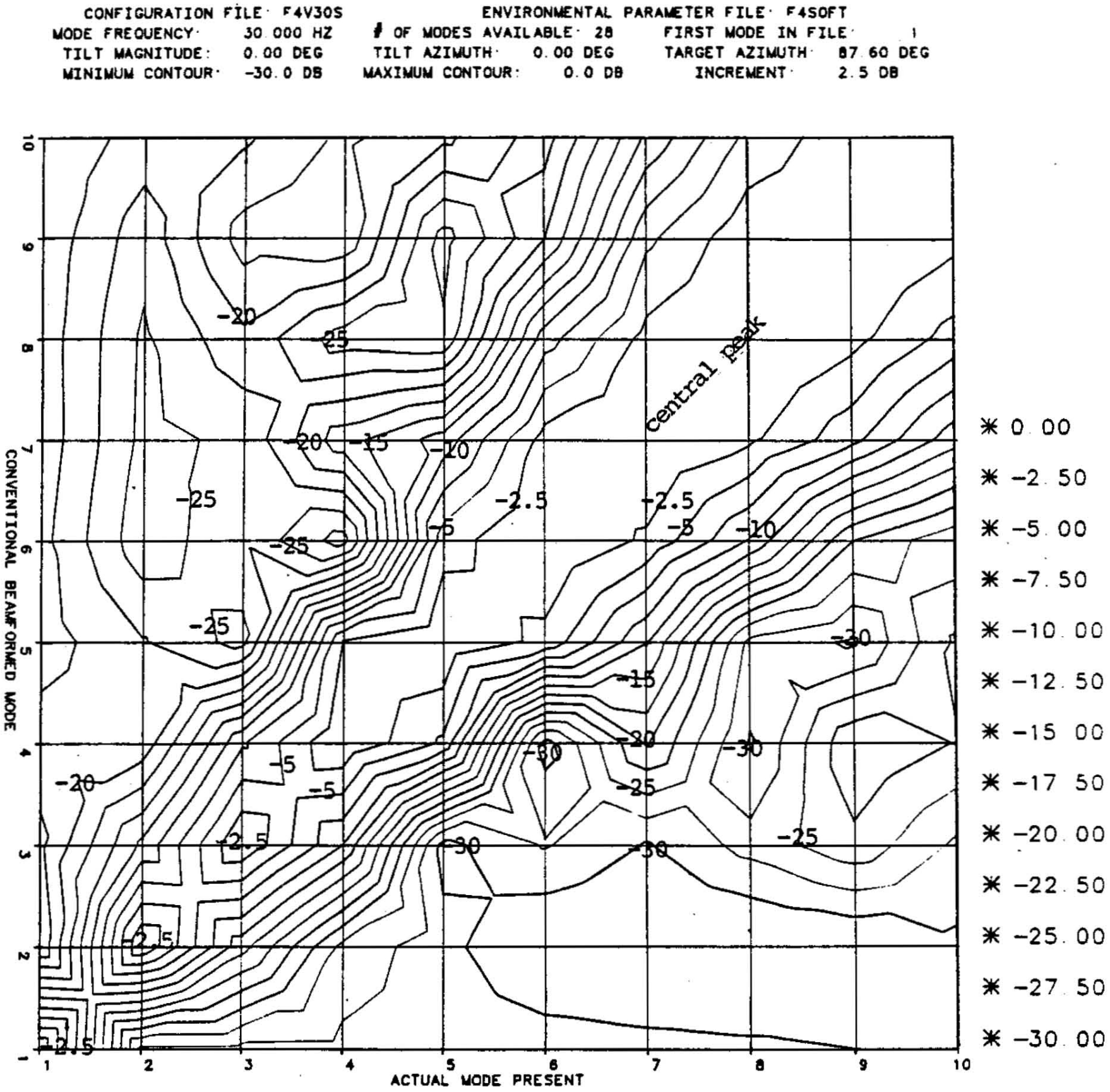

Figure 5-1: Theoretical Modal Sidelobe Pattern - $30 \mathrm{~Hz}$ Single Beam Least Squares Beamformer for 10 Modes (all 18 elements) 
obtained when the array is steered to that particular mode. However, it must be stressed that the mode domain differs from the bearing domain by being fundamentally discrete in nature. Thus, for this and all other plots having one or more axes indexed in mode number, the results shown at non-integer values of mode number are only linear interpolations between the true data, which exists only for integer mode numbers. This technique of displaying results is used so that large quantities of information may be displayed in a compact manner. To emphasize the discrete nature of the data, a dark grid has been added to the plot at integer values of the mode number.

The calculations displayed in Figure 5-1 are theoretical in that they have been computed with perfectly known mode shapes, sensor positions, and sensor (russcoherence matrices. In particular, complications caused by the substitution of an estimate for the actual $\overline{\underline{\mathbf{s}}}_{\mathrm{N}}$ matrix have not been considered. The cross-coherence matrix used in the computation of the figure consists of one mode of unit amplitude in background noise that is spatially white, i.e.

$$
\overline{\underline{\mathbf{S}}}_{\mathrm{N}}=\underline{\mathbf{E}}_{i} \underline{\mathbf{E}}_{i}^{+}+\lambda\left|\underline{\mathbf{E}}_{i}\right|^{2} \underline{\underline{\mathbf{I}}}
$$

where

$$
\left|\underline{\mathbf{E}}_{i}\right|^{2}=\underline{\mathbf{E}}_{i}^{+} \underline{\mathbf{E}}_{i}
$$

Here $\underline{E}_{i}$ is the steering vector of the mode indicated by the horizontal axis. Thit is. it is the particular column of the steering matrix $\underline{\underline{E}}$ defined in equation (1.4) that describes the mode assumed to be present in the signal field. $\lambda$ is the inverse of the effective modal signal-to-noise ratio; a value of $10^{-3}$ has been used in the generation of Figure 5-1.

A beamformer of perfect resolution would create a ridge of $0 \mathrm{~dB}$ outputs alongthe major diagonal of the plot. This ridge would be of narrow eross-section, with 

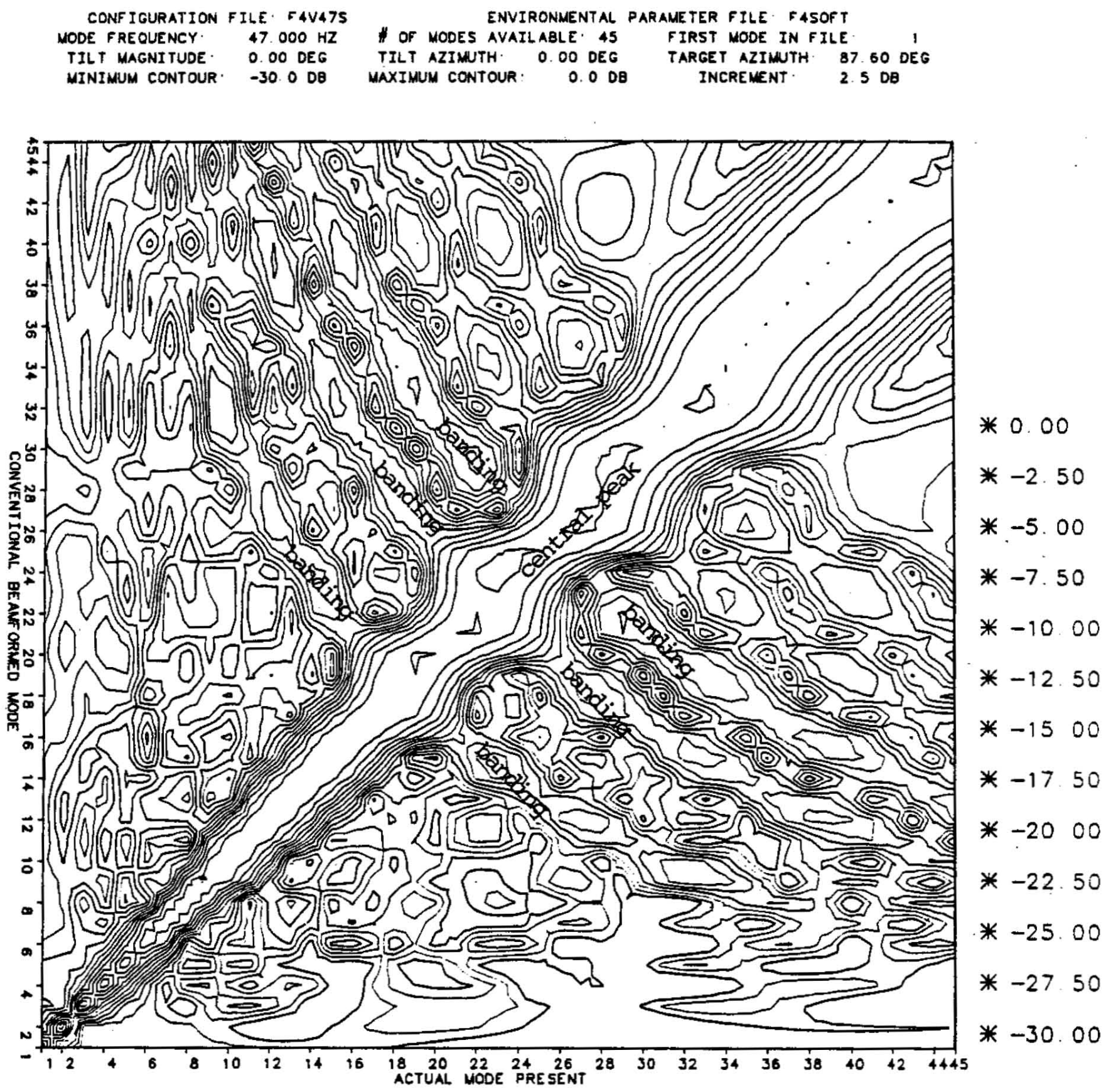

Figure 5-2: Theoretical Modal Sidelobe Pattern - $47 \mathrm{~Hz}$ Single Beam - Least Squares Beamformer for 45 Modes (all 18 elements) 
CONFIGURATION FILE. FSV47S MODE FREOUENCY. $47.000 \mathrm{HZ}$

TILT MAGNITUDE. 0 OO DEG MINIMUM CONTOUR: $\quad-30.0 \mathrm{OB}$
ENVIRONMENTAL PARAMETER FILE. FASOFT OF nODEs available. 45 TILT AZIMUTH. O. OO DEG MAXIMUN CONTOUR. $0.0 \mathrm{OB}$
FIRST NOOE IN FILE

TARGET AZIMUTH. OT 00 DEC INCREMENT . 2.500

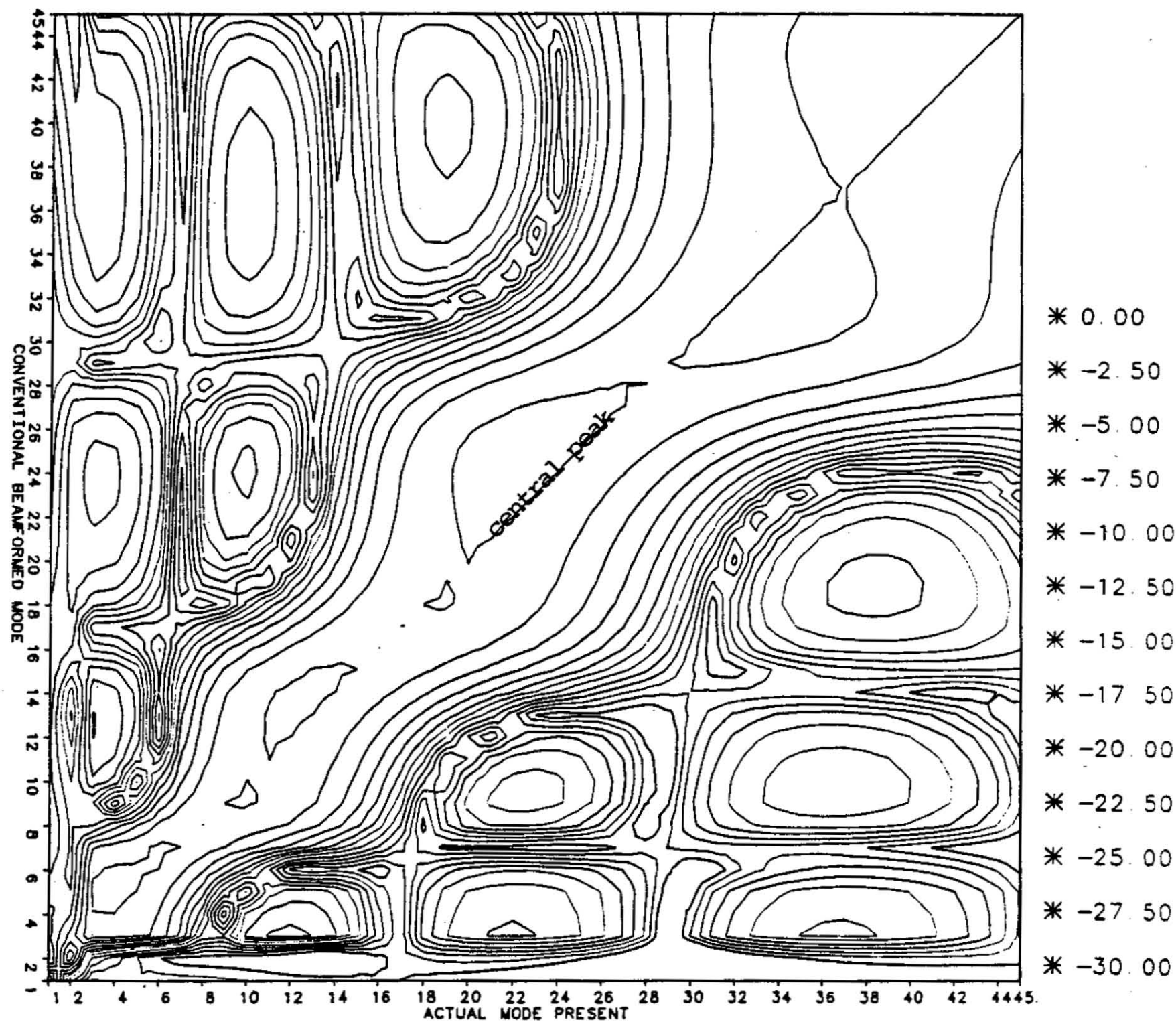

Figure 5-3: Theoretical Modal Sidelobe Pattern - $47 \mathrm{~Hz}$ Single Beam Least Squares Beamformer for 45 Modes (top 9 elements only) 

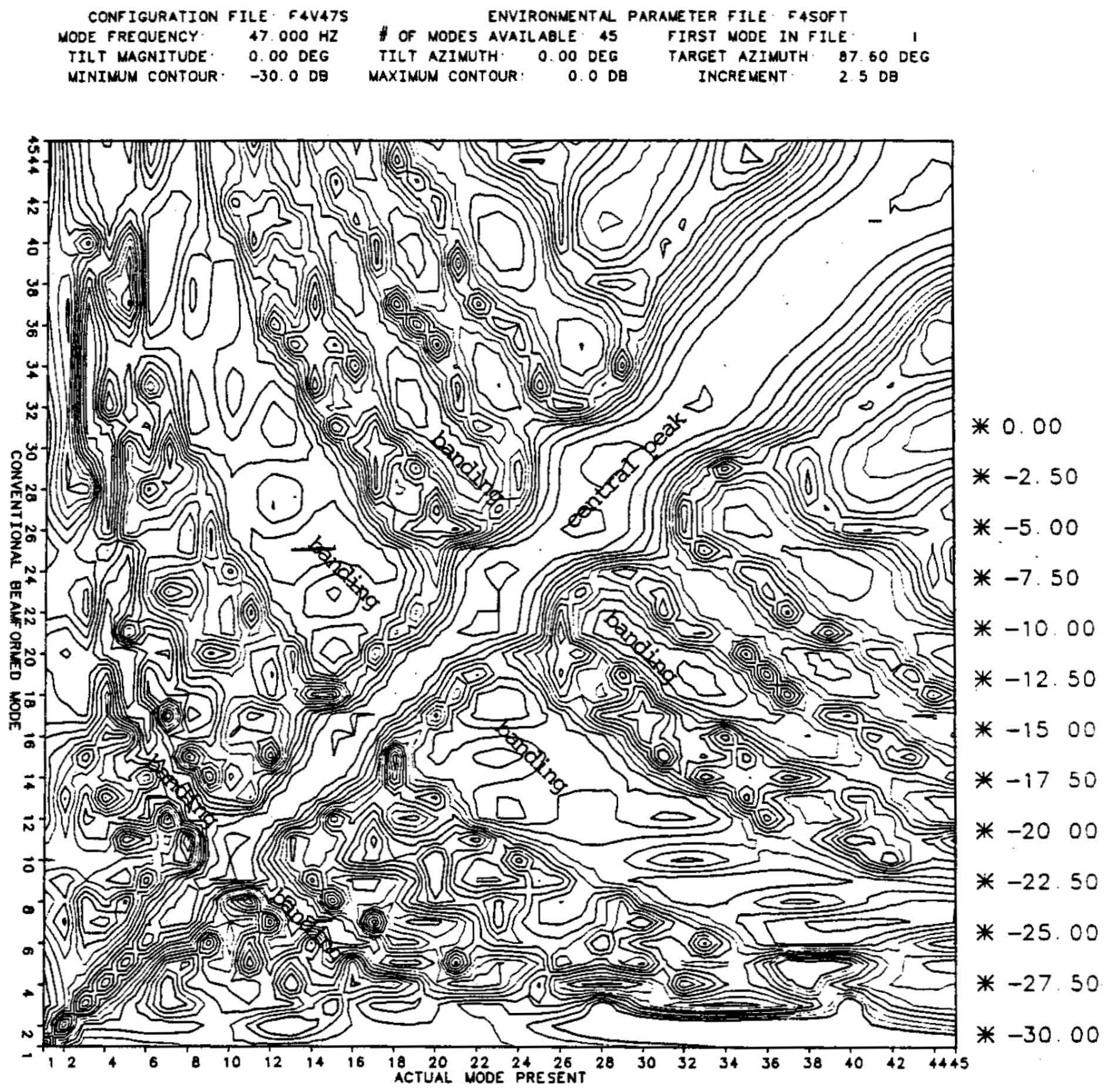

Figure 5-4: Theoretical Modal Sidelobe Pattern - $\mathbf{4} \mathbf{7} \mathrm{Hz}$ Single Beam Least Squares Beamformer for 45 Modes (every other element only) 
the output immediately falling to a floor of about $-30 \mathrm{~dB}$ (the level of the white noise included in the cross-coherence matrix) for any off-diagonal points in the grid. Figure 5-1 shows quite graphically that the single beam least squares modal beamformer is far from ideal at $30 \mathrm{~Hz}$ when applied to the FRAM IV vertical array. The first mode is clearly resolved; about $20 \mathrm{~dB}$ of separation is observed between it and the second mode. To lesser degrees, modes 2 and 3 can also be separated, with about $-10 \mathrm{~dB}$ of leakage between modes 2 and 3 , and about $-7 \mathrm{~dB}$ between modes 3 and 4. However, all modes beyond the third can be seen to be fundamentally unresolvable with the available array. For example, the sidelobe between modes 4 and 5 is, at best, down by only $3 \mathrm{~dB}$; hence, energy from either mode 4 or mode 5 is capable of generating large output levels when the beamformer is steered to mode 4 . It can be concluded that the single beam least squares modal beamformer is theoretically capable of resolving no more than 3 modes at $30 \mathrm{~Hz}$. The realities of dealing with actual data can be expected to degrade performance still further.

Similar investigations at other frequencies indicate that the algorithm can resolve only a single mode at $15 \mathrm{~Hz}, 5$ modes at $47 \mathrm{~Hz}$, and only about 8 modes even at $71 \mathrm{~Hz}$. Figure 5-2 displays the results for the 18 elements of the FR.AM IV vertical array at $47 \mathrm{~Hz}$. Note that the range of modes displayed in Figure $\mathbf{5}-\mathbf{2}$ extends to 45 versus the 10 displayed in Figure 5-1. This is done to demonstrate the different forms of aliasing that are possible. The major modal sidelobes observed in Figure 5-2 can be divided into two types. One type is a general broadening of the central ridge with increasing mode number. The other type is a general banding that occurs at right angles to the central ridge, and is prominent at higher mode numbers.

The broadening of the central peak sidelobe structure in Figure 5-2 can be attributed to the problem of inadequate array length, as shown in Figure 5-3. This 
figure has been generated from the $47 \mathrm{~Hz}$ mode set in a fashion identical to that used in the creation of Figure 5-2, except that the bottom 9 elements in the array have been excluded from the beamformer, effectively halving the array aperture. The resultant large increase in the width of the central peak is readily observed.

In contrast, Figure 5.4 has been recreated from Figure 5-2 by eliminating every other recorded sensor, rather than the bottom nine. The number of sensors has again been halved, but now in such a way as to retain the total array aperture. As expected, the width of the central peak in Figure 5-4 is comparable with that of Figure 5-2. However, the banding observed at the higher modes has now moved down in mode number. This is clearly due to an inadequate sensor population in the available array aperture. Note that the pattern does not occur until one or both of the injected mode number and the beamformed mode number is greater than the number of sensors in the vertical array (18 in Figure 5-2 and 9 in Figure 5-4).

To better understand the causes of the two types of aliasing, one must first consider how the beamformer works in a physical sense. In essence, the single beam least squares algorithm uses the elements of the vertical array to effect a finite sum approximation of the mode orthogonality integral (equation (2.8)). The amount of aliasing is an inverse measure of the effectiveness of this sum in approximating the integral. Too great an inter-sensor spacing results in undersampling the aperture of the array, which, in turn, causes aliasing similar to that generated when a time series is sampled below the Nyquist rate. This type of aliasing generally occurs between modes of significantly different mode number, as is demonstrated by the banding effect it generates. On the other hand, a vertical array aperture of inadequate length can cause significant portions of some of the mode shapes involved to be excluded from the integration, also causing anomalous results. In this case, though, it is generally modes that are close in mode number that look 
most similar; hence, this type of aliasing primarily contributes by widening the central ridge of figures 5-1 through 5-4. A study of the mode shapes presented in Figures 4-4 through 4-7 verifies this conclusion. At every frequency, the first mode not resolved by the single beam least squares algorithm is also the first to have a significant portion of its mode shape extend below the $960 \mathrm{~m}$ length of the array. In some respects, the two types of aliasing are also akin to the local versus global error problems encountered in target detection and estimation theory [82].

From the above discussion, it becomes obvious that, in the frequency range of interest, the modal resolution available from the FRAM IV vertical array is fundamentally limited by its length. At the lowest frequencies available in the data set, not more than a single mode can be resolved by the conventional spatial matched-filter; even at the highest frequencies in the data set, only about $10^{\circ} \%$ of the modes that might contribute significantly to the received field can be resolved. Such performance is clearly inadequate, requiring consideration of higher resolution modal beamforming methods.

\subsection{Performance of the MLM Beamformers}

\subsubsection{Performance of the Single Beam MLM Beamformer for Incoherent Modes}

When applied to plane wave beamforming, the MLM algorithm is normally considered to be a high resolution alternative to least squares (conventional) beamforming. From a theoretical viewpoint, a similar interpretation can be made for modal beamforming. This is illustrated in Figure 5-5. The contour plot displayed in this figure is identical to that of Figure 5-1, except for the algorithm employed in computing the side lobe patterns. Here, it-is the theoretical ontput of the single beam MLM algorithm at $30 \mathrm{~Hz}$ that is shown, rather than the output of 

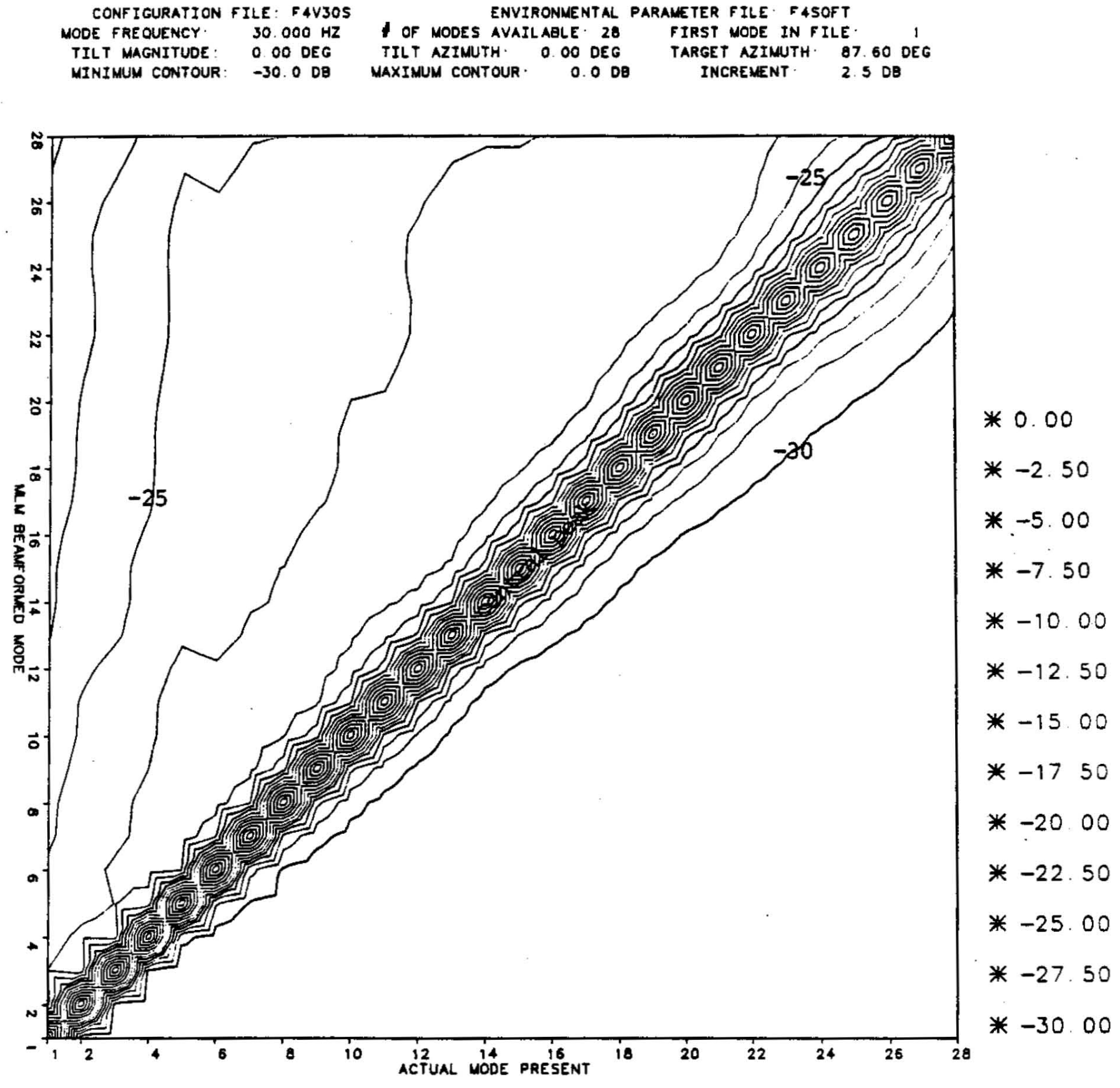

Figure 5-5: Theoretical Modal Sidelobe Pattern - $30 \mathrm{~Hz}$ Single Beam MLM Beamformer for 28 Modes (all 18 elements) 
the least squares algorithm displayed earlier. The same SNR as used earlier (30 dB) has been assumed in the generation of this figure.

The result displayed in Figure 5-5 is very close to that of an ideal modal beamformer, and provides the motivation for investigating the MLM algorithm in modal decomposition applications. However, practical issues involving the implementation of the processor typically limit this ideal theoretical performance. In particular, Figure 5-5, like Figures 5-1 through 5-4, has been computed using a perfectly known sensor cross-coherence matrix. To measure the the effect of using only an estimate of the $\underline{\underline{\mathbf{S}}}_{\mathrm{N}}$ matrix, sets of synthetic data have been created and processed using the single beam MLM algorithm. The processed output for one of these data sets is displayed in Figure 5-6. This figure is once again a contour plot with the surface height representing modal amplitude on a $\mathrm{dB}$ scale. Here, though. the horizontal axis represents time, while the vertical axis represents the mode number to which the beamformer is steered. The plot consists of the time series over 20 minutes of the amplitude estimate for each of the 18 modes displayed. These estimates have been melded together to form the contoured surface by linear interpolation between adjacent modes. As was the case earlier, the only real data in the vertical occurs at integer values of the mode number. The contoured format is used only because it allows information to be displayed at a high visual density.

To interpret the results displayed in Figure 5-6, one must first understand the details involved in the creation of the data set. The sound field has been synthesized from the $30 \mathrm{~Hz}$ mode shapes presented in Chapter 4, with each of the odd modes between the first and the fifteenth contributing to the sum with unit amplitude. Since only the odd modes are included in the sound field. the even mode amplitude outputs indicate the amount of energy that the algorithm allows to leak into their estimates. To better differentiate between various modes in the output. 


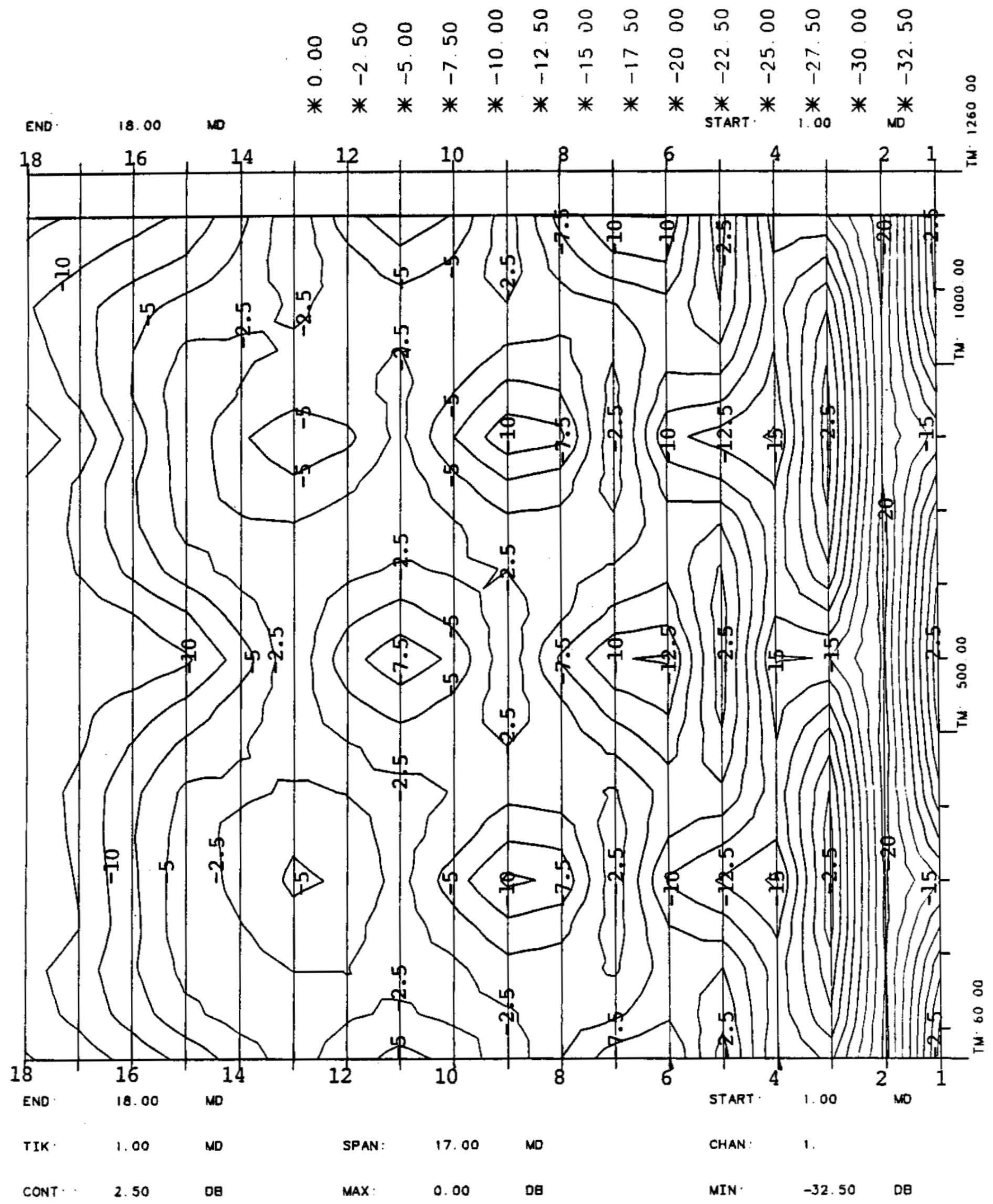

$30 \mathrm{HZ}$ - SINGLE BEAM MLM BEAMFORMER - INCOHERENT SYNTHETIC DATA

Figure 5-6: Output of Single Beam MLM Beamformer versus Time for Incoherent Synthetic Data at $30 \mathrm{~Hz}$ 
the unit amplitudes have been modulated by sinusoids with periods of 20 minutes: the full time extent of the plot corresponds to exactly one period of the modulation. The starting point of the modulating sinusoid has been adjusted by $90^{\circ}$ for each succeeding odd mode, so that the modulation for mode 1 looks like a cosine function, while the modulation for mode 3 appears as a sine function, etc. Each of the various modal contributions has been provided with an independent random phase component, so that they are phase random with respect to each other. The resulting field can then be considered to be a sum of incoherent modes. The random phase fluctuations have been created with a temporal correlation length of $32 \sec (20$ points), so as to approximate the effect of the final preprocessing filter. A small amount of spatially uncorrelated white noise (at about $30 \mathrm{~dB}$ SNR) is included in each hydrophone's time series.

The synthetic data set has been created in a format identical to that of the preprocessed real data; namely, a complex time series sampled every 1.6 seconds for each recorded sensor. The beamforming itself has been computed using: an averaging window of 2 minutes for $\underline{\hat{\bar{S}}}_{\mathrm{N}}$, representing about 4 degrees of freedom. with updates once a minute (for a $50 \%$ overlap factor). The resulting estimate of the sensor cross-coherence matrix is subsequently stabilized for inversion by the addition of a small positive constant to the diagonal terms.

In Figure 5-6, the first mode is clearly resolved. The amplitude modulation is apparent, demonstrating both the proper period and the correct initial phase. The output for the second mode indicates that the processing provides more than $20 \mathrm{~dB}$ of rejection between it and modes modes 1 and 3 . Similarly, all modes through the seventh are well resolved, and modes 8 through 11 can be at least partially distinguished. This performance certainly exceeds that of the single beam least squares algorithm, even without considering the effects of an using only an estimite 
of $\overline{\underline{S}}_{\mathrm{N}}$ on the latter.

\subsubsection{The Relationship Between the Single and Multiple Beam Variants of the MLM Beamformer for Incoherent Modes}

Because Hinich [42] has proposed a multiple beam MLM algorithm for modal beamforming, it is important to consider the relationship of the two algorithms in the case of incoherently summed modes. To see the nature of the relationship. one must review briefly the technique by which the MLM algorithm assures maximum signal-to-noise ratios at the beamformer output. From a linear algebra perspective. the problem consists of determining $N$ complex unknowns, each representing the complex gain applied to the observed field at a given sensor. The problem then contains $N$ degrees of freedom. For the single beam case, the MLM result is obtained by effecting a constrained minimization of the noise power. The constraint guarantees undistorted passage of the desired signal through the beamforming: process, and uses one degree of freedom. The remaining $N-1$ degrees are then employed to minimize the power of the noise in which the signal is embedded. This minimization may be visualized as an optimal placement of $N-1$ nulls in the modal beam pattern.

By comparison, the multiple beam variant can be considered to have $M . N$ degrees of freedom, representing the unknown complex gains to be applied at any given sensor for each of $M$ beams. Cursory consideration of the problem shows that at least $M$ distortionless constraints (one for each beam) are required. A more detailed investigation shows that $M^{2}$ constraints are actually placed upon the problem. The extra constraints represent decoupling requirements between the different beams. These are needed to guarantee accurate energy accounting: without them, energy detected on one beam could also leak throngh to other beams. 
and thus be accounted for multiple times. Thus, there are a total of $M \cdot(N-. M$ degrees of freedom available for minimizing the noise on $M$ beams, or only $N-M$ degrees of freedom for each beam. The multiple beam MLM algorithm can then be viewed as the equivalent of single beam result with some extra decoupling constraints included. These extra constraints have the effect of locating a priori some of the nulls that would otherwise be free for noise minimization.

The upshot of this rather abstract discussion is that one always obtains better SNR performance from the single beam form of the MLM algorithm than from the multiple beam variant when processing an incoherently summed mode field. This is obvious, since the multiple beam approach requires prior placement of nulls that would otherwise be located to optimally reject noise; this prior null placement can never improve the total noise rejection. A full and mathematically rigorous argument to the same effect is presented in Appendix B. One simple indication of this behavior is that when $M=N$, both the least squares and MLM algorithms reduce to the same solution

$$
\underline{\overline{\mathbf{S}}}_{\mathrm{M}}=\overline{\overline{\mathbf{E}}}^{-1} \underline{\overline{\mathbf{s}}}_{\mathrm{N}}\left(\underline{\overline{\mathbf{E}}}^{+}\right)^{-1},
$$

where the steering matrix $\underline{\overline{\mathrm{E}}}$ is now $N \times N$ and may be assumed to be non-singular. Such a result is expected, since under these conditions all the available degrees of freedom are utilized as constraints, leaving none for the noise minimization that is the source of the MLM algorithm's superior performance.

The conclusion to be drawn from Figures 5-1 through 5-6 and the previous discussion is that the single beam MLM modal beamformer is the algorithm of choice for vertical array data modal decomposition when the sound field consists of incoherent modes. This finding must be tempered by the results of the following section, which show that it is very sensitive to how well the incoherent mode assumption is met in practice. 


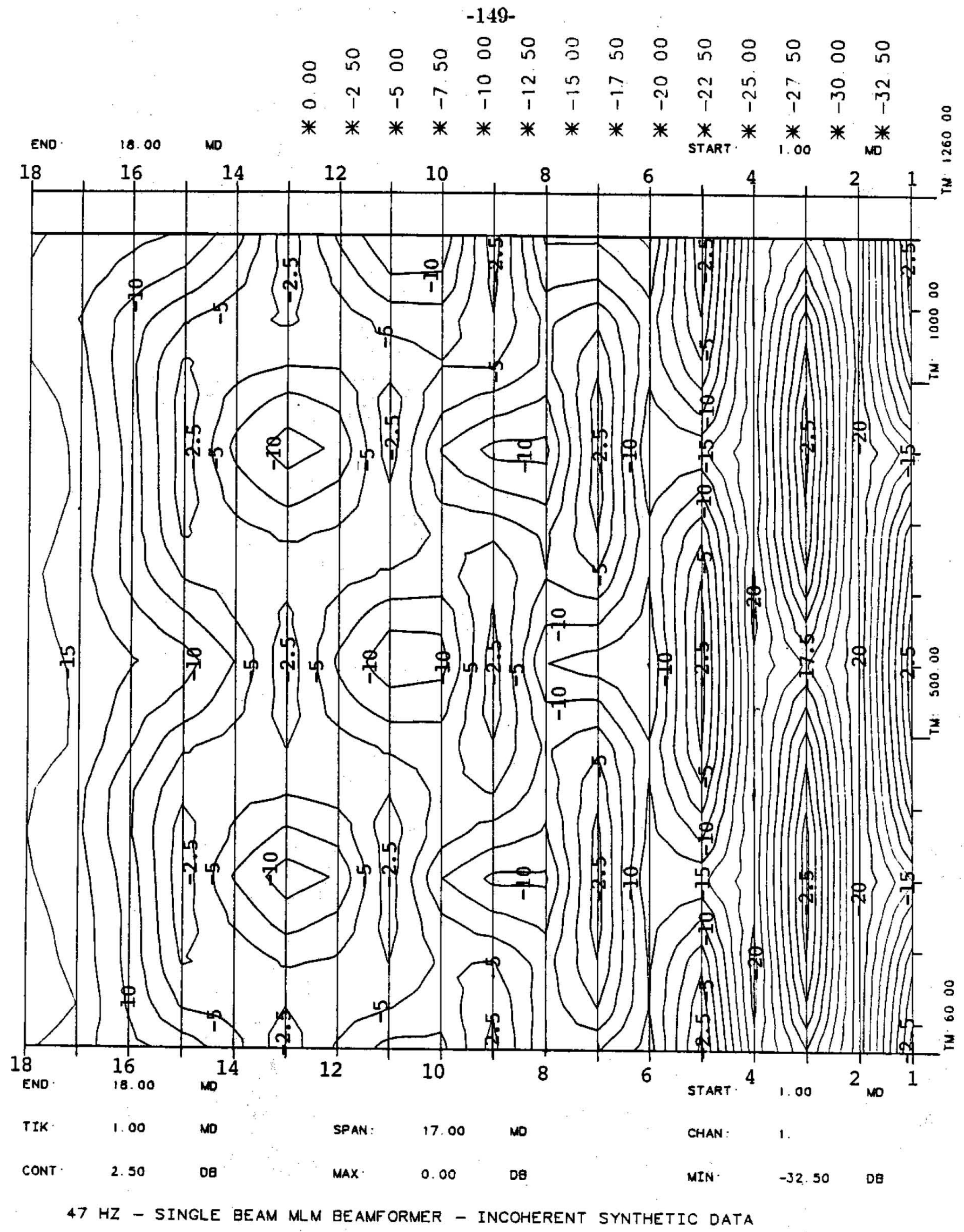

Figure 5-7: Output of Single Beam MLM Beamformer versus Time for Incoherent Synthetic Data at $\mathbf{4 7} \mathrm{Hz}$ 


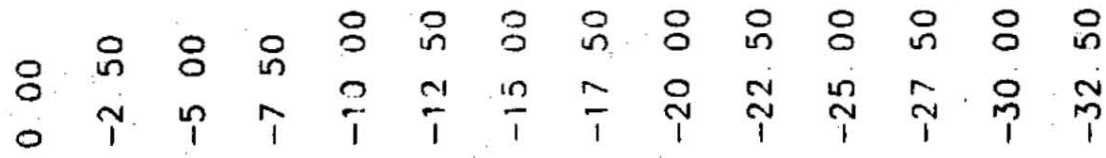

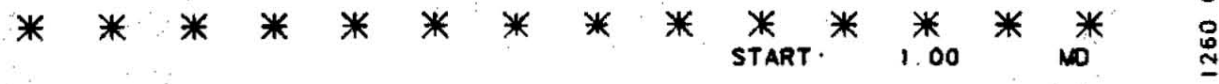
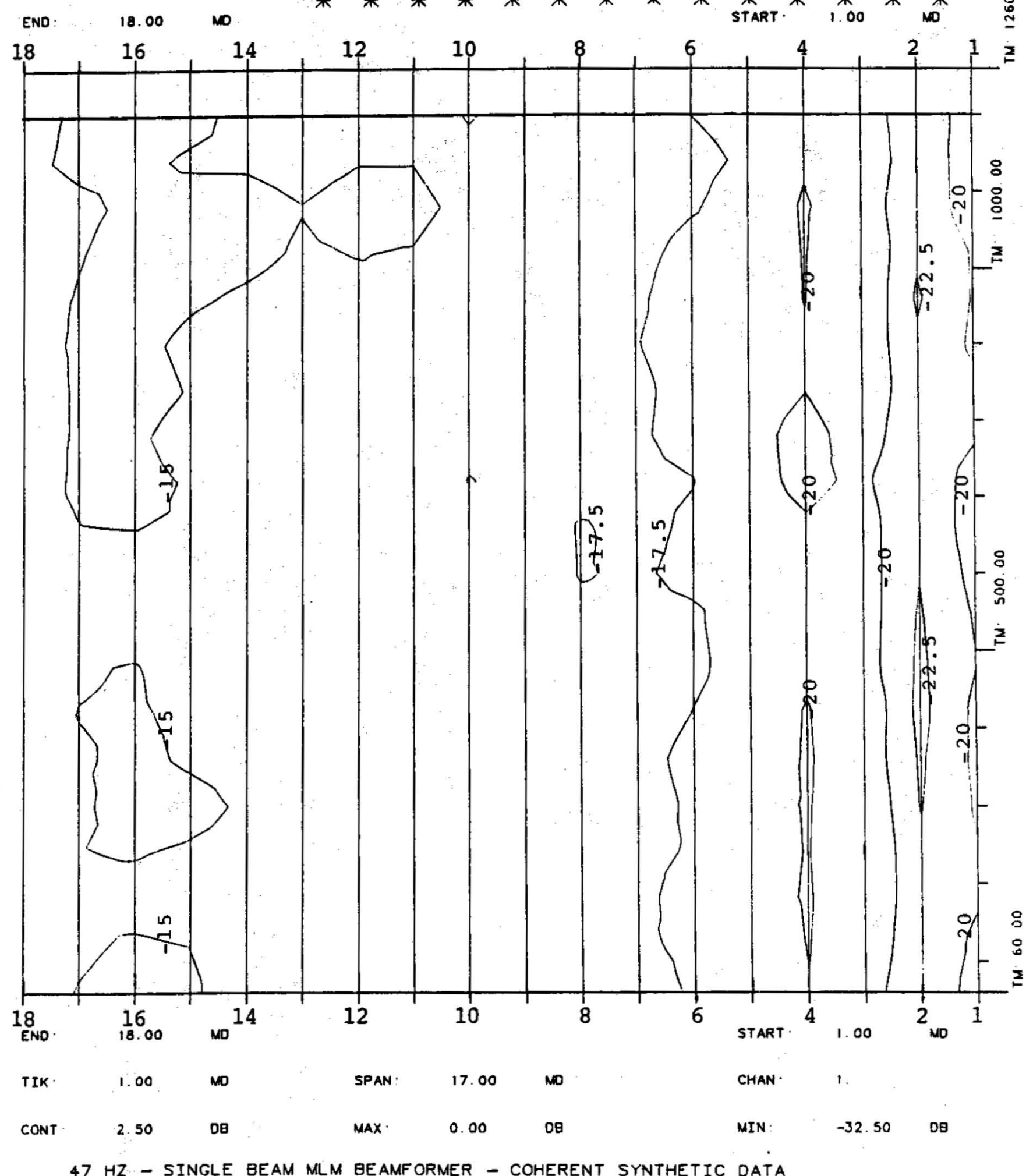

Figure 5-8: Output of Single Beam MílM Beamformer versus Time for Coherent Synthetic Data at $47 \mathrm{~Hz}$ 


\subsubsection{Performance of the Single Beam MLM Algorithm for Vertical Arrays in the Presence of Coherent Interference}

As has already been indicated in Chapter 3 and is conclusively demonstrated in Chapter 7 , the various complex mode amplitudes are highly coherent in the FRAM IV data set. This mode coherence has a significant effect on the performance both of the MLM algorithms, making neither the single beam processor nor the multiple beam processor applicable to the present problem. It is revealing to study this problem in some detail, so that the effects of mode coherence on the MLM algorithm can be better understood.

Figure 5-7 displays the output of the single beam MLM modal beamformer for a synthetic sound field at $47 \mathrm{~Hz}$. In all other particulars except frequency, both the synthetic data generation and the processing is identical to that of Figure 5-6. Most. importantly, the synthetic field once again consists of the odd modes between 1 and 15 combined in a temporally phase random fashion, so that the various modal contributions can be considered to be incoherent. Each mode is once more assigned an amplitude of unity and sinusoidally modulated. At $47 \mathrm{~Hz}$, the first 9 modes are easily resolved, and all of the modes through mode 15 (the last one present in the signal field) are at least partially resolved.

Like Figure 5-7, Figure 5-8 also displays the output of the single beam MILII modal beamformer for a synthetic sound field at $47 \mathrm{~Hz}$. Both the synthetic data generation and the processing is identical to that of both Figure 5-6 and Figure 5- 7 . with one important difference; while the modal contributions to the sound field in Figures 5-6 and 5-7 are summed incoherently by injecting random phise fluctuations that vary independently from mode to mode, the modal contributions for Figure 5-8 are summed in a coherent fashion. This is accomplished by injecting a phase fluctuations that are constant across all modes, so that a constant phisse 
relationship is maintained over time between the various modal amplitudes. In striking contrast to the results of Figure 5-7, the resulting output from the single beam MLM beamformer for this case is so poor as to be essentially meaningless.

Having demonstrated numerically that the issue of mode coherence is of extremely important in determining the performance of MLM modal beamformers, it is next important to develop a more intuitive understanding of the problem. Consider a sound field consisting of a single mode in spatially white noise. Let the the complex amplitude of the mode that is present be $a_{p}$ and the steering vector associated with it be denoted by $\underline{\mathbf{E}}_{p}$. This modal steering vector is the particular column of the steering matrix $\underline{\overline{\mathbf{E}}}$ defined in equation (1.4) that describes the mode assumed to be present in the signal field. The sensor cross-coherence matrix for such a situation is

$$
\overline{\mathbf{S}}_{\mathrm{N}}=\left|a_{p}\right|^{2} \underline{\mathbf{E}}_{p} \underline{\mathbf{E}}_{p}^{+}+\lambda\left|a_{p}\right|^{2}\left|\underline{\mathbf{E}}_{p}\right|^{2} \overline{\underline{\mathbf{I}}} \text {, }
$$

where $\lambda$ is the inverse of the effective modal signal-to-noise ratio and

$$
\left|\underline{\mathrm{E}}_{p}\right|^{2}=\underline{\mathbf{E}}_{p}^{+} \underline{\mathbf{E}}_{p}
$$

The single beam MLM beamformer estimates the squared magnitude of the complex amplitude of the mode to which it is steered, $\left|\hat{a}_{b}\right|^{2}$, as

$$
\left|\hat{a}_{b}\right|^{2}=\frac{1}{\left(\underline{\mathbf{E}}_{b}^{+} \underline{\overline{\mathbf{S}}}_{\mathbf{N}}^{-1} \underline{\mathbf{E}}_{b}\right)}
$$

In contrast to $\underline{\mathbf{E}}_{p}$, which is the steering vector of the mode actually present in the signal field, $\underline{\mathbf{E}}_{\dot{b}}$ is the steering vector of the mode to which the beamformer is steered. Using the identity given in equation (A.3), it is possible to compute the inverse of $\overline{\mathbf{S}}_{\mathrm{N}}$ as given by equation (5.28). This result may then be substituted into equation (5.30) to yield 


$$
\left|\hat{a}_{b}\right|^{2}=\left(\frac{\left|a_{p}\right|^{2}\left|\underline{\mathbf{E}}_{p}\right|^{2}}{\left|\underline{\mathbf{E}}_{b}\right|^{2}}\right) \frac{\lambda(1+\lambda)}{1+\lambda-\left|\rho_{b p}\right|^{2}} \text {. }
$$

Here $\rho_{b p}$ is the normalized inner product between $\underline{\mathbf{E}}_{b}$ and $\underline{\mathbf{E}}_{p}$

$$
\rho_{b p}=\frac{\underline{\mathbf{E}}_{b}^{+} \underline{\mathbf{E}}_{p}}{\left|\underline{\mathbf{E}}_{b}\right|\left|\underline{\mathbf{E}}_{p}\right|}
$$

The magnitude of $\rho_{b p}$ always lies in a range from 0.0 to 1.0 .

Figure 5-5 is nothing more than equation (5.31) evaluated at various combinations of $\underline{\mathbf{E}}_{b}$ and $\underline{\mathbf{E}}_{p}$. It is easy to see how the MLM beamformer obtains its high resolution. When the beamformer is steered to the mode that is present in the field, $\rho_{b p}$ takes on a value of 1.0 , leading to an output of

$$
\left|\hat{a}_{p}\right|^{2}=\left|a_{p}\right|^{2}(1+\lambda) \text {. }
$$

On the other hand, when the mode present in the signal field is different from the mode to which the beamformer is steered, the value of $\rho_{b p}$ is small if the two modes are well resolved by the array. In fact, for the limiting case of a vertical array of continuous aperture extending across the full sound channel, $\rho_{b p}$ is guaranteed to be zero for all possible mode pairs, since the numerator of equation (5.32) approaches the orthogonality integral of equation (2.8). Thus, the squared magnitude of $\rho_{b p}$ is generally negligible for well resolved modes, leading to

$$
\left|\hat{a}_{b}\right|^{2} \approx \lambda\left(\frac{\left|a_{p}\right|^{2}\left|\underline{\mathbf{E}}_{p}\right|^{2}}{\left|\underline{\mathbf{E}}_{b}\right|^{2}}\right) .
$$

Since the value of $\lambda$ is small for high SNR signals, (in Figure 5-5, a value of $10^{-3}$ is used), excellent rejection is obtained when the mode present is not the one to which the beamformer is steered.

In the case where the sound field consists of two modes whose contributions 
are incoherent with respect to each other, the sensor cross-coherence matrix can be written as

$$
\overline{\underline{\mathbf{S}}}_{\mathrm{N}}=\left|a_{p 1}\right|^{2} \underline{\mathbf{E}}_{p 1} \underline{\mathbf{E}}_{p 1}^{+}+\left|a_{p 2}\right|^{2} \underline{\mathbf{E}}_{p 2} \underline{\mathbf{E}}_{p 2}^{+}+\lambda\left|a_{p 1}\right|^{2}\left|\underline{\mathbf{E}}_{p 1}\right|^{2} \underline{\mathbf{I}}
$$

where $\underline{\mathbf{E}}_{p 1}$ and $\underline{\mathbf{E}}_{p 2}$ are the steering vectors of the two modes contributing to the signal field. Here, for simplicity, the white noise scaling has been kept identical to that of equation (5.28). Equation (5.35) can also be inverted and then substituted into (5.30) to obtain a general result. In this case, it is of the most interest to study the effect of the presence of the second mode on the beamformer estimate for the first mode, which can be obtained by setting $\underline{\mathbf{E}}_{b}$ equal to $\underline{\mathbf{E}}_{p 1}$. This leads to

$$
\left|\hat{a}_{p 1}\right|^{2}=\left|a_{p 1}\right|^{2}\left(1+\frac{\lambda(b \lambda+1)}{(b \lambda+1)-\left|\rho_{p 1 p 2}\right|^{2}}\right),
$$

where

$$
b=\frac{\left|a_{p 1}\right|^{2}\left|\underline{\mathbf{E}}_{p 1}\right|^{2}}{\left|a_{p 2}\right|^{2}\left|\underline{\mathbf{E}}_{p 2}\right|^{2}}
$$

Again, it is easy to see how high resolution is obtained; if the two modes present are well resolved by the array, then the squared magnitude of $\rho_{p 1 p 2}$ is once more negligible, giving an output of

$$
\left|\hat{a}_{p 1}\right|^{2} \approx\left|a_{p 1}\right|^{2}(1+\lambda)
$$

This result is identical to that of equation (5.33), which means that the single beam MLM beamformer almost completely eliminates the effect of the presence of the second incoherent mode in the signal field. Such a conclusion is certainly in agreement with the results of Figures $5-6$ and 5-7.

The ability of the single beam MLM algorithm to discriminate against the presence of the second mode is inherently tied to the ability of the array to resulve 
the two modes. If the two modes are well resolved, then $\left|\rho_{p 1 p 2}\right|^{2}$ is small. and therefore justifiably negligible. On the other hand, if the two modes are fundamentally unresolvable, then ignoring this term is a poor approximation at best. In either case, though, the performance is at least as good as that of the single beim least squares beamformer.

To highlight the difference between case where the the signal modes combine incoherently and the case where they remain phase locked with respect to each other, the preceding problem is now repeated for a sound field consisting of two modes that sum coherently. In this situation, the sensor cross-coherence matrix is

$$
\begin{gathered}
\overline{\mathbf{S}}_{\mathrm{N}}=\left(a_{p 1} \underline{\mathbf{E}}_{p 1}+a_{p 2} \underline{\mathbf{E}}_{p 2}\right)\left(a_{p 1} \underline{\mathbf{E}}_{p 1}+a_{p 2} \underline{\mathbf{E}}_{p 2}\right)^{+}+ \\
\lambda\left|a_{p 1}\right|^{2}\left|\underline{\mathbf{E}}_{p 1}\right|^{2} \underline{\overline{\mathbf{I}}}
\end{gathered}
$$

Again, the white noise scaling has been kept identical to that of equation (5.28). Comparing equations (5.35) and (5.39), it can be seen that the effect of the modes being coherent is to force the retention of cross-terms that would average to zero in the incoherent case. Inversion of equation (5.39) and subsequent substitution into (5.30) is easily accomplished. Upon steering the beamformer to the first mode, one obtains

$$
\left|\dot{a}_{p 1}\right|^{2}=\frac{\left|a_{p 1}\right|^{2} \lambda(b \lambda+c)}{(b \lambda+1)-\left|\rho_{p 1 p 2}\right|^{2}}
$$

where $b$ is again defined as in equation (5.37) and $c$ is

$$
c=\frac{\left|a_{p 1} \underline{\mathbf{E}}_{p 1}+a_{p 2} \underline{\mathbf{E}}_{p 2}\right|^{2}}{\left|a_{p 2}\right|^{2}\left|\underline{\mathbf{E}}_{p 2}\right|^{2}} .
$$

Assuming as before that the modes are well resolved by the array, $\left|\rho_{p 1 p_{2}}\right|^{2}$ (an again be neglected, so that for typical white noise levels the output is approximately 


$$
\left|\hat{a}_{p 1}\right|^{2} \approx c\left(\lambda\left|a_{p 1}\right|^{2}\right)
$$

The resulting output is now proportional to $\lambda$. If both the modal steering vectors are assumed to be of roughly equal norm, and if the modal amplitudes are approximately equal, then $c$ can take on values of between 0.0 and 4.0 , depending on the relative phase between the two modes and how well they are resolved by the array. For well resolved modes, $c$ approaches a value of 2.0 , since the steering vectors associated with the modes are close to orthogonal. On the other hand. equation (5.34) demonstrates that the background noise floor that leaks into any mode is approximately $\lambda\left|a_{p 1}\right|^{2}$ under the assumption of equal norms for different modal steering vectors. Thus, the presence in the signal field of two coherent, well resolved modes of roughly equal strength causes the single beam MLM algorithm to generate output estimates of these modes that are only $3 \mathrm{~dB}$ above the noise floor, no matter how strong the actual modal signals. This is exactly the phenomenon seen in Figure 5-8.

Further investigation of equation (5.40) yields two significant conclusions. First, even very small levels of a second coherent mode can trigger the interference effect. Again assuming that the modes are well resolved, consider the limit as $a_{p 2}$ vanishes. For this case, $c$ approaches the value of $b$, and equation (5.40) then simplifies to

$$
\left|\hat{a}_{p 1}\right|^{2} \approx\left|a_{p 1}\right|^{2} \frac{b \lambda}{b \lambda+1}
$$

The result approaches the correct value of $\left|a_{p 1}\right|^{2}$ only if $b \lambda$ is much greater than 1.0. indicating that coherent contributions from other modes are negligible to the MLM processor only if they are at or below the background noise level. Second, the effect of coherent interference on the single beam MLM algorithm is worse when the two 
modal steering vectors are well resolved than it is when they are poorly resolved. In the limit as $\underline{\mathbf{E}}_{p 2}$ approaches $\underline{\mathbf{E}}_{p 1},\left|\rho_{p 1 p 2}\right|^{2}$ takes on a value of unity, and the output becomes a very reasonable

$$
\left|\hat{a}_{p 1}\right|^{2} \approx\left|a_{p 1}+a_{p 2}\right|^{2}
$$

This is exactly the opposite of what might be intuitively expected. Beamforming algorithms typically experience greater difficulty when multiple sources look very similar than when they are easily distinguished. But the nature of equation (5.10) is such that more realistic results are achieved from the single beam MILM algorithm when the value of $\left|\rho_{p 1 p 2}\right|^{2}$ is near 1.0 (indicating that the interfering nodes are poorly resolved) than when it is close to 0.0 (meaning that the interfering modes are well resolved).

For the ideal case of a continuous vertical aperture extending across the full channel, the single beam MLM approach is completely inappropriate when the modes contributing to the signal field remain coherent. This is because mode orthogonality guarantees that $\rho_{p 1 p 2}$ is always zero, a situation which leads to the worst possible performance of the MLM processor. As will be investigated in the sequel, its suitability for use with horizontal arrays is somewhat better.

The fundamental reason that coherently interfering modes cause the single beam MLM algorithm difficulty is that they violate the assumption of statistical independence between signal and noise. In the single beam approach, the signal. by definition, is the mode selected for detection; any other modes present are considered to be part of the noise against which the processor is to discriminate. If the modes are phase random with respect to each other, then the selection of one mode as the signal while the others are included in the noise causes no violation of this fundamental assumption. On the other hand, the definition of a single mode as 
the signal destroys the assumption of independence between signal and noise when the modes remain coherent. The effect of the resulting coherent interference is to corrupt the spatial structure of the signal mode. The beamformer then rejects the energy carried in the signal mode because the mode is no longer recognizable. This is why equation (5.42) indicates that the output is roughly the same as background noise levels.

\subsubsection{Performance of the Multiple Beam MLM Algorithm for Vertical Arrays in the Presence of Coherent Interference}

Since the single beam MLM algorithm is clearly inappropriate for the task at hand, the effect of coherent modal interference on the multiple beam variant is now investigated. From the discussion in Sections 5.2 and 5.3 , it can be seen that the fundamental advantage of the multiple beam approach is that it expands the number of modes which are simultaneously designated as signal from 1 to $M$, the number of beams included in the multiple beam beamformer. If a multiple beam MLM algorithm that includes all modes making significant coherent contributions to the total signal field is implemented, then the statistical independence of signal and noise can be reestablished, and the resulting algorithm should, in theory, produce acceptable results. A number of practical considerations limit the applicability of this approach, though.

The issue of greatest importance is the proper selection of $M$, the number of modes to include in the beamformer. As mentioned earlier, this choice is basically a modeling decision, since the inclusion of extra modes in the beamformer is tantamount to improving the initially assumed signal model. Including an insufficient number of modes leaves one with the same problem that plagues the single beam approach; the algorithm performs poorly because the signal and the 
noise, as implicitly defined by the model selection, are not statistically independent. On the other hand, making $M$ too large is also detrimental, for two reasons. The original derivation limits the number of modes that can be handled simultaneously to the number of sensors available. Otherwise, $\underline{\underline{\mathbf{E}}}^{+} \overline{\mathbf{S}}_{\mathrm{N}}^{-1} \overline{\mathbf{E}}$ is of less than full rank, and therefore uninvertible.

For the FRAM IV vertical array (and many other vertical arrays), the fact that many of the higher order modes cannot be adequately resolved places an even more restrictive limit on $M$. Since two modes become unresolvable when the steering vectors describing them become too similar, the inclusion of multiple unresolvable modes in the steering matrix quickly drives it towards rank deficiency, so that inversion of the quadratic product creates serious difficulties from the viewpoint of numerical stability. The impact of this issue on the multiple beam least squares algorithm is studied in some detail in Section 5.9. Its effect on the multiple beam MLM algorithm is similar, limiting the effective number of beams that can be handled simultaneously to as few as 3 or 4 below $20 \mathrm{~Hz}$, and no more than perhaps a dozen even at $71 \mathrm{~Hz}$.

It has been shown previously that any mode contributing coherently to the signal field in an amount that exceeds the background noise level must be considered significant (see equation (5.43)). Thus, one reaches the conclusion that it is not possible to implement a multiple beam MLM processor capable of dealing with the coherent interference problem for the FRAM IV vertical array. However. given a sufficiently improved array, such an approach could be possible. The primary remaining issue under these conditions would be one's ability to invert large dimension arrays (perhaps $30 \times 30$ or $50 \times 50$ ) in a numerically stable fashion. The array necessary to support such processing would need to extend much further in depth, so that it could adequately resolve significantly greater 
numbers of modes. Only a relatively modest increase in the number of hydrophones would be needed. The addition of further sensors beyond this amount would be beneficial, though, since the performance improvement that MLM algorithms over equivalent least squares algorithms is fundamentally linked to the size of $N-M$ (the excess of sensor count over beam count).

\subsubsection{Alternative Implementations of the MLM Algorithm in the Presence of Coherent Interference}

Alternative implementations of the MLM algorithm in the presence of coherent interference are possible if one is not interested in actually measuring individual modal characteristics. While these approaches are generally not applicable to the present problem, it is of interest to understand their relationship to it.

Consider again the sensor cross-coherence matrix for two coherently interfering modes (equation (5.39)). A consideration of the eigenvalues and eigenvectors of this matrix quickly leads to the conclusion that the output of the single beam MLM beamformer is maximized when a steering vector of the form

$$
\underline{\mathbf{E}}_{b}=k\left(a_{p 1} \underline{\mathbf{E}}_{p 1}+a_{p 2} \underline{\mathbf{E}}_{p 2}\right)
$$

is used [20]. Here $k$ is an arbitrary scaling factor that would typically be used for normalization of the steering vector. This observation forms the basis for all these alternative implementations. If the relative mode amplitudes and phases were known a priori, then an optimal detector could be created by beamforming to a steering vector which was the properly weighted sum of the individual modal steering vectors. In this thesis, of course, these are exactly the parameters to be measured, but there may be ways of predicting them accurately enough to allow significant improvements in detection processing. 
One alternative approach is to index the beamforming across a set of steering vectors generated by various weighted sums of the modal steering vectors; arbitrary variations of the modal amplitudes and phases become impractical beyond a very few modes, however. A more promising variant is to index the beamforming across the two parameters of source range and depth, using one or another of the different propagation theories currently available to predict the mode amplitudes and phases needed to compute the sum. The advantage of such a method is that source range and depth are immediately available once detection is made. Several preliminary efforts in this direction show promise. The work of Fizell and Wales [34] is of special note, since it applies the approach to actual field data.

\subsubsection{Performance of MLM Algorithms for Horizontal Arrays in the Presence of Coherent Interference}

After much effort, two important conclusions have been reached concerning the use of the MLM algorithm for reduction of the FRAM IV vertical array data set. The first is that the MLM algorithm works very well when the modes making up the sound field are incoherent. For this situation, the single beam variant is guaranteed to provide better performance than any multiple beam variant. The second is that the MLM algorithm has a great deal of difficulty when the modes comprising the sound field remain coherent with respect to each other. In this case. the performance is worst when the coherently interfering modes are well resolved by the array rather than poorly resolved, as might otherwise be expected. The difficulties that arise when coherent interference is encountered are severe enough to make both the single and multiple beam versions unattractive for use with the vertical array data. On the other hand, the MLM approach can still be used on the horizontal array data if adequate care and caution are exercised. It is revealing to investigate why better performance might be expected of the single beam MLM 


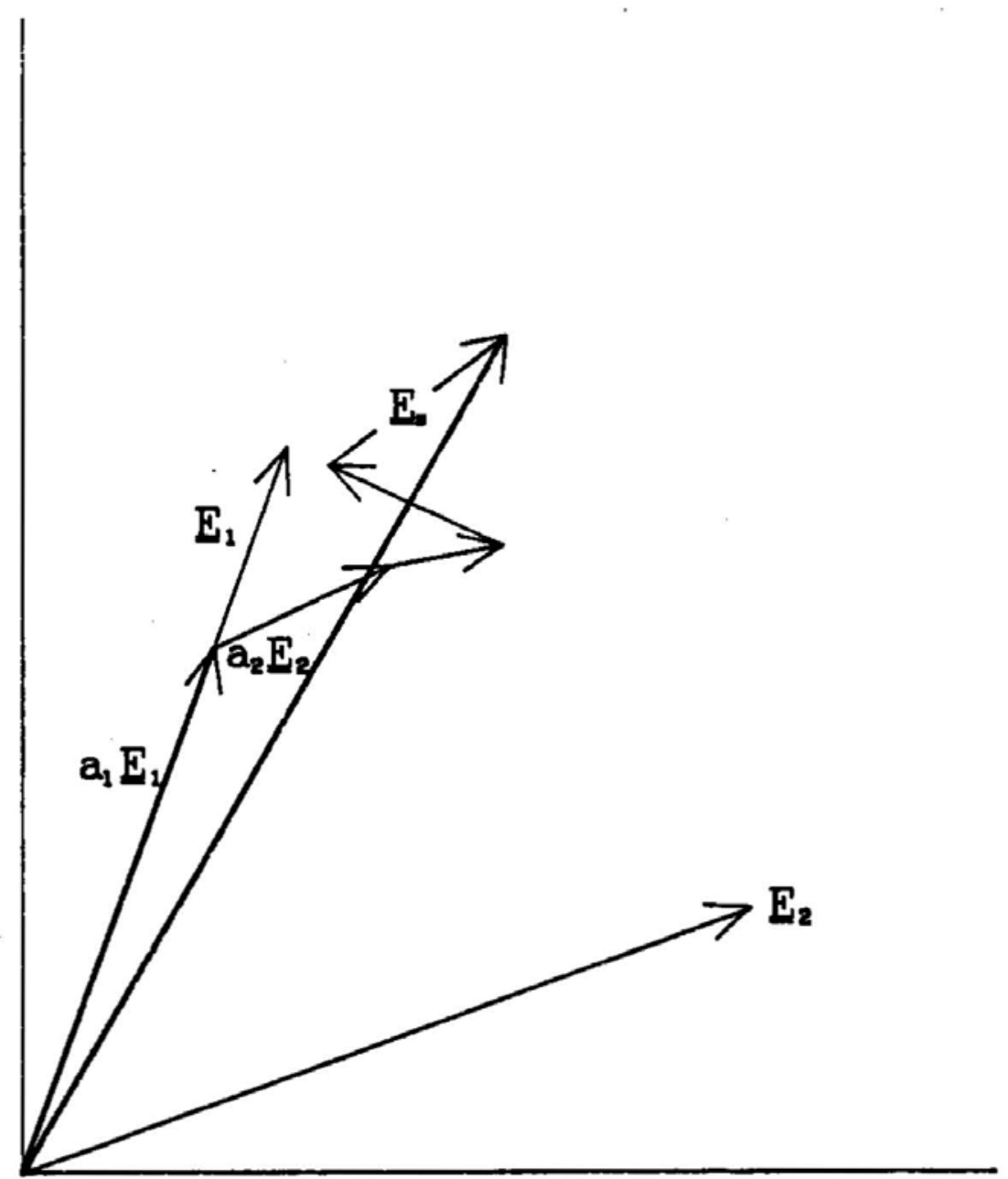

Figure 5-9: Components of a Coherent Sound Field for a Notional Horizontal Array

beamformer in a coherent mode field when it is applied to horizontal array dital than when it is used with vertical array data.

Consider a situation where a horizontal array is receiving a large number of coherent modal arrivals. Let the steering vector associated with the $k^{\text {th }}$ mode be $\underline{\mathbf{E}}_{k}$, and its complex amplitude be denoted by $a_{k}$. The sensor cross-coherence matrix 
is then of the form

$$
\overline{\mathbf{S}}_{\mathrm{N}}=\underline{\mathbf{E}}_{s} \underline{\mathbf{E}}_{s}^{+}+\sigma^{2} \underline{\overline{\mathbf{I}}}
$$

where $\underline{\mathbf{E}}_{s}$ is a weighted sum the steering vectors associated with the various modes that are present

$$
\underline{\mathbf{E}}_{s}=\sum_{k} a_{k} \underline{\mathbf{E}}_{k}
$$

This sum is shown ${ }^{k}$ pictorially in Figure 5-9. As discussed in Section 5.4, the modal steering vectors for a horizontal array are identical to the steering vectors for plane waves, with the exception of a scalar equal to the size of the mode shape at the array's deployment depth. The equivalent plane waves are all traveling in the same horizontal direction but with slightly different horizontal phase speeds. Under these conditions, the modal steering vectors can be expected to be almost parallel to both one another and to the total sum. Figure 5-9 has been drawn to reflect this situation.

Assume that the single beam MLM beamformer is steered to the $i^{\text {th }}$ mode, Its output can then be evaluated directly from equation (5.31) by setting $a_{p}=1$. $\underline{\mathbf{E}}_{p}=\underline{\mathbf{E}}_{s}$, and $\underline{\mathbf{E}}_{b}=\underline{\mathbf{E}}_{i}$. Since $\underline{\mathbf{E}}_{s}$ and $\underline{\mathbf{E}}_{i}$ are almost parallel, it is not unreasonable to represent their inner product as

$$
\left|\rho_{i s}\right|^{2}=1-\epsilon_{i s},
$$

where $\epsilon_{i s}$ is small with respect to unity but still large when compared to $\lambda$. The case where $\epsilon_{i s}$ is the same or smaller than $\lambda$ is not of interest, since this generally corresponds to operational situations where the array is fundamentally unable to resolve the modal structure (such as a horizontal line array operated near broadside). The amplitude estimate that the beamformer makes for the $i^{\text {th }}$ mode is then 


$$
\left|\hat{a}_{i}\right|^{2}=\left(\frac{\left|\underline{\mathbf{E}}_{s}\right|^{2}}{\left|\underline{\mathbf{E}}_{i}\right|^{2}}\right) \frac{\lambda}{\epsilon_{i s}} .
$$

Although this output is still proportional to the noise level $\lambda$, the effect is greatly mitigated by the small denominator $\epsilon_{i s^{\prime}}$ which is typically on the order of $10^{-1}$ or $10^{-2}$.

The result presented in equation (5.49) raises the very important question of why the resultant output should bear any resemblence to the actual modal amplitude distribution. It is not at all obvious why the estimate should be large when the actual mode amplitude is large and why it should be small when the mode amplitude is small. Of the four terms on the right hand side of equation (5.49). only the two in the denominator depend upon the mode to which the beamformer is steered. The variation of $\left|\underline{\mathbf{E}}_{i}\right|^{2}$ with mode number is only a scalar related to the size of the mode's shape at the array depth, and, hence, of no practical consequence. Indeed, if the steering vector of the equivalent plane wave is instead considered. even this simple variation with mode number is eliminated, and the term can be considered to be constant. Thus, the change in output level with mode number is principally caused by the $\epsilon_{i s}$ term, so that the estimator output tracks the actual modal amplitude distribution only if $\epsilon_{i s}$ is generally small for modes of large amplitude and big for modes of small amplitude. While this is never guaranteed to be the case, a graphical argument can be made to support the conclusion that it is often so.

Consider once more the sum of equation (5.47) and its graphical depiction shown in Figure 5-9. $\underline{\mathbf{E}}_{8}$ can be expected to lie in a direction that is closest to the direction of the modes providing the largest contributions. For these modes, then. $\epsilon_{i s}$ is generally smaller than average, providing the desired peak in the modial 
amplitude distribution. The effect is most reliable when a single modal contribution dominates all others, as is often the case in the Arctic. This situation is shown in Figure 5-9. In creating this figure, the lengths of the various modal steering vectors have been assumed to be roughly equal, and the amplitude of mode 1 has been assumed to be much greater than the amplitude of any other mode. For this situation, it is apparent that the angle between $\underline{E}_{3}$ and $\underline{E}_{1}$ is smaller than the angle $\underline{\mathbf{E}}_{s}$ makes with other modal steering vectors.

It is the natural sensitivity of equation (5.49) to variations in $\epsilon_{i s}$ that provides the MLM algorithm with better resolution than the conventional beamformer. For comparison, the output of the single beam least squares beamformer is approximately

$$
\left|\hat{a}_{i}\right|_{L S}^{2}=\left(\frac{\left|\underline{E}_{s}\right|^{2}}{\left|\underline{E}_{i}\right|^{2}}\right)\left(1-\epsilon_{i s}\right) .
$$

While this result provides a much better absolute level than equation (5.49), its form is such as to make it significantly less sensitive to variations in $\epsilon_{i s}$ than the MLM result.

Thus, the single beam MLM beamformer ends up providing the desired result of higher resolution than the conventional beamformer when applied to modal separation with horizontal arrays, although not for the reasons typically given to explain its operation. Additionally, the linkage through $\epsilon_{i s}$ is highly non-linear. making both absolute levels and relative peak levels highly suspect, although positions of the peaks in mode number (or, equivalently, grazing angle or phase speed) can generally be accepted as accurate. In previous cases where the MLMI algorithm has been used in the presence of multiple coherent sources, or with a single coherent source in a stable multi-path environment, these effects have probably been incorrectly attributed to the effect of the MLM bias discussed earlier. 
The distinction is important, because in many situations one can accurately compensate for the bias effect, whereas the extraction of reliable levels in the presence of coherent interference is impossible.

In conclusion, the MLM algorithm still provides higher resolution in the presence of coherent interference than conventional processing does when applied to horizontal array data, despite the fact that the various arrivals are highly coherent. This higher resolution is achieved at the expense of accurate peak levels and a marked decrease in the robustness of the processing, though. The results are most trustworthy in the case of one strong modal arrival dominating all other coherent contributions to the signal field. All of these effects can be seen in the resultant outputs of the MLM processor that are presented in Chapter 7 .

\subsection{Performance of the Multiple Beam Least Squares Beamformer}

Since neither form of the MLM algorithm can be reliably applied to the FRAM IV vertical array data in a modal beamforming context, the multiple beam least squares beamformer must then be considered as a high resolution alternative. Such an approach offers several advantages. First, the multiple beam algorithm can be expected to provide at least marginally better performance than the single beim variant if the number of modes included in the beamformer is not excensive. Because the multiple beam algorithm essentially attempts to fit the observed pressure field with a model containing a greater number of parameters, closer agreement with the data generally results. Additionally, since the derivation of the multiple beam least squares algorithm requires no assumption about the statistical relationship between the signal and the noise, its performance is not degraded by: coherent interference. It also provides some relief from the inter-modal aliasing problems seen in the single beam least squares algorithm, although the improvement 


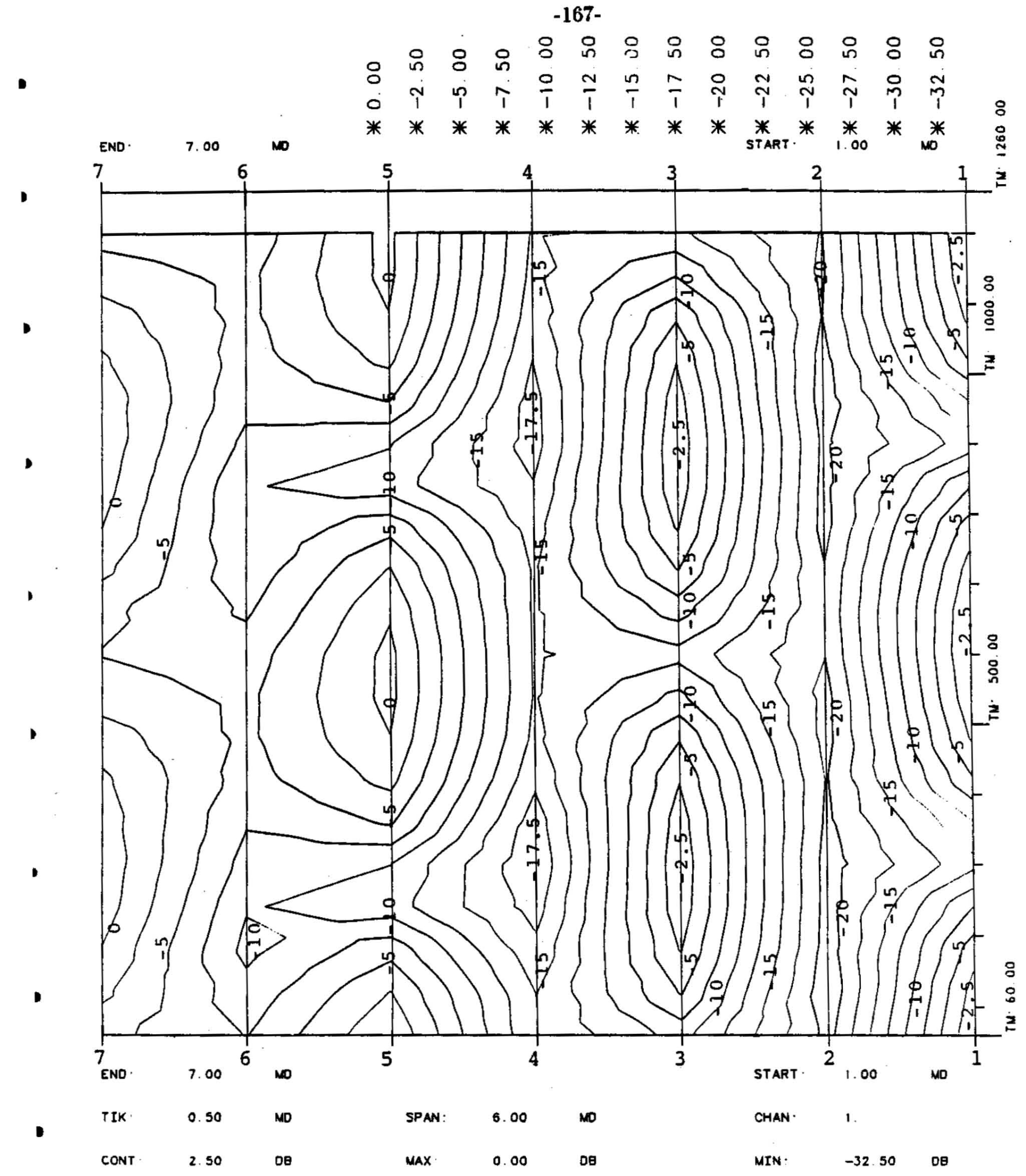

$47 \mathrm{HZ} \mathrm{-} \mathrm{MULTIPLE} \mathrm{BEAM} \mathrm{LEAST} \mathrm{SQUARES} \mathrm{BEAMFORMER} \mathrm{-} \mathrm{COHERENT} \mathrm{SYNTHETIC} \mathrm{DATA}$

Figure 5-10: Output of Multiple Beam Least Squares Beamformer versus Time for Coherent Synthetic Data at $47 \mathrm{~Hz}$

( 7 modes included in beamformer) 


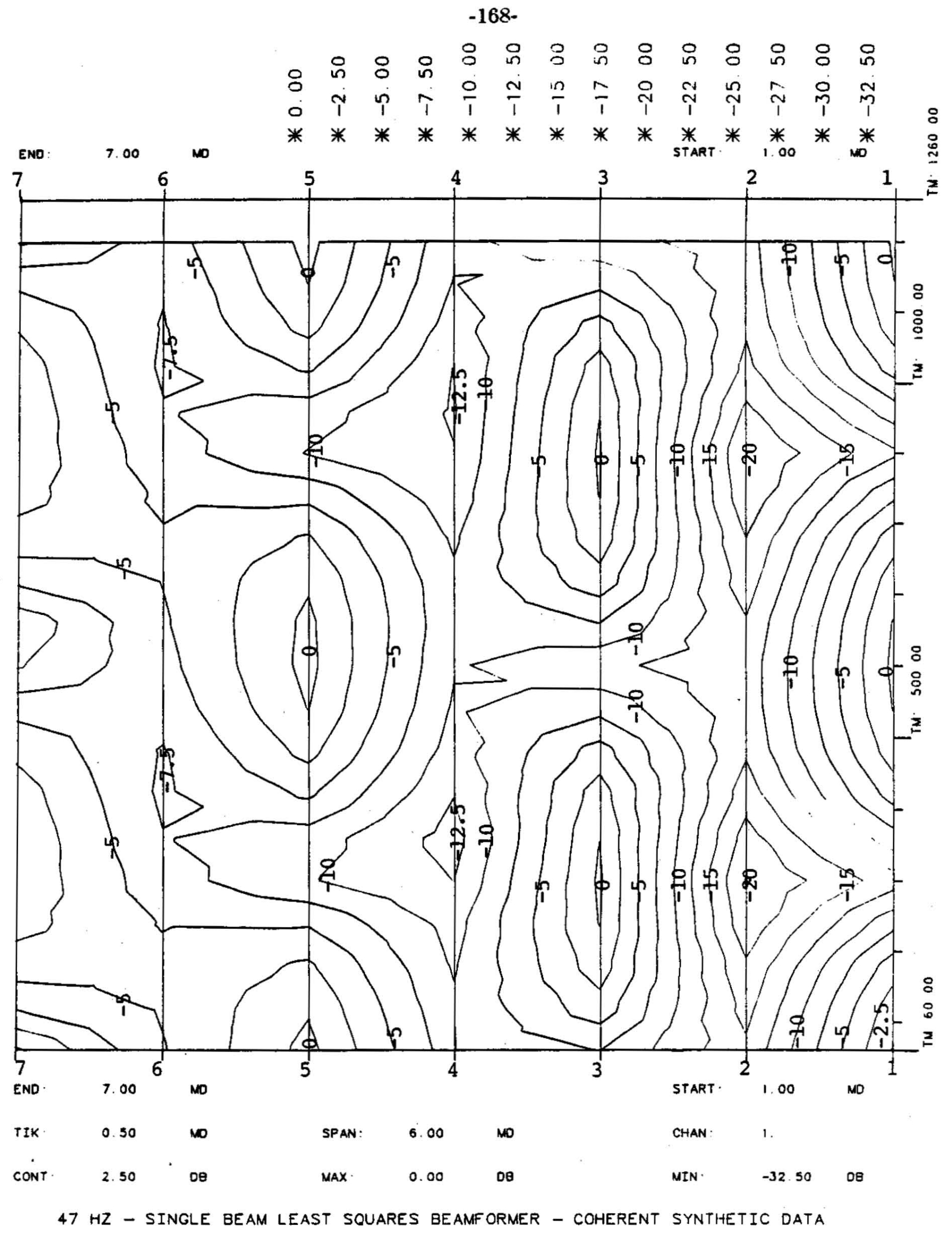

Figure 5-11: Output of Single Beam Least Squares Beamformer versus Time for Coherent Synthetic Data at $17 \mathrm{~Hz}$

(7 modes shown) 
is not as great as that which the single beam MLM algorithm would provide if it could be applied. Finally, the multiple beam version is somewhat more computationally efficient than the single beam variant.

A typical set of modal amplitude estimates generated by the multiple beam least squares approach is presented in Figure 5-10. For comparison, the equivalent set of outputs from the single beam variant is presented in Figure 5-11. Similar to Figures 5-6 through 5-8, the modal amplitudes are depicted as contours over a surface consisting of time on the horizontal axis and mode number on the vertical axis. As mentioned earlier, the only real data points occur for integer values of the mode number; results plotted at non-integer mode values are merely interpolations between adjacent data points.

The particulars of the synthetic data sets used to generate both of these figures are identical to those of the earlier figures. Again, the synthetic signal field consists of a coherent sum of the odd modes between 1 and 15, each with unit amplitude. The various modal amplitudes have once more been alternatively modulated with cosine and sine waveforms with periods of 20 minutes. Both plots span the first seven modes at $47 \mathrm{~Hz}$. All seven are resolvable in Figure 5-10. About $20 \mathrm{~dB}$ of rejection is observed in the second mode and about $17 \mathrm{~dB}$ is seen in the fourth mode. These results show some improvement over those of Figure 5-11. where only the lower order modes can effectively be resolved, and where rejection in the second and fourth mode troughs is somewhat less.

While the additional resolution provided by the multiple beam least squares algorithm is not great, it is significant. This is especially true at frequencies below $20 \mathrm{~Hz}$, where the single beam approach has difficulty resolving more than one mode. It is for this reason that the multiple beam least squares beamformer has been selected as the most attractive alternative for the modal decomposition of the 
FRAM IV vertical array data set.

\subsection{Determination of the Number of Modes to Model}

The final topic to be addressed in this chapter is the proper choice of $M$, the number of modes to be included in the multiple beam algorithm. Ideally, the choice would include any mode likely to provide a significant contribution to the total sound field seen at the receiver. However, the inclusion of an excessive number of modes in the beamformer can lead to problems involving numerical stability, as can be seen in Figure 5-12. In this figure, the same $47 \mathrm{~Hz}$ synthetic data used to generate Figures 5-10 and 5-11 has been reprocessed with a multiple beam least squares algorithm that includes the first eight modes instead of the seven used above. By increasing $M$ from seven to eight, the effective resolution has been decreased rather than increased; only four modes are now actually resolved. while the rest are saturated by processing-induced noise.

To understand this effect, consider the output of the multiple beam least squares processor for an input consisting only of spatially white noise. For such a case, the sensor cross-coherence matrix is

$$
\overline{\mathbf{S}}_{\mathrm{N}}=\sigma^{2} \underline{\overline{\mathbf{I}}} \text {. }
$$

From equation (5.8), the output of the processor can then be evaluated as

$$
\underline{\hat{\mathbf{S}}}_{\mathrm{M}}=\sigma^{2}\left(\underline{\overline{\mathbf{E}}}^{+} \underline{\overline{\mathbf{E}}}\right)^{-1} \text {. }
$$

It is obvious that as the $M \times M$ matrix $\underline{\overline{\mathbf{E}}}^{+} \underline{\overline{\mathbf{E}}}$ becomes singular, one or more outputs of the processor grows without bound. Numerical instability in the inversion can then make the algorithm extremely sensitive to even small amounts of background noise. Therefore, a proper choice of $M$ must be based on a compromise 


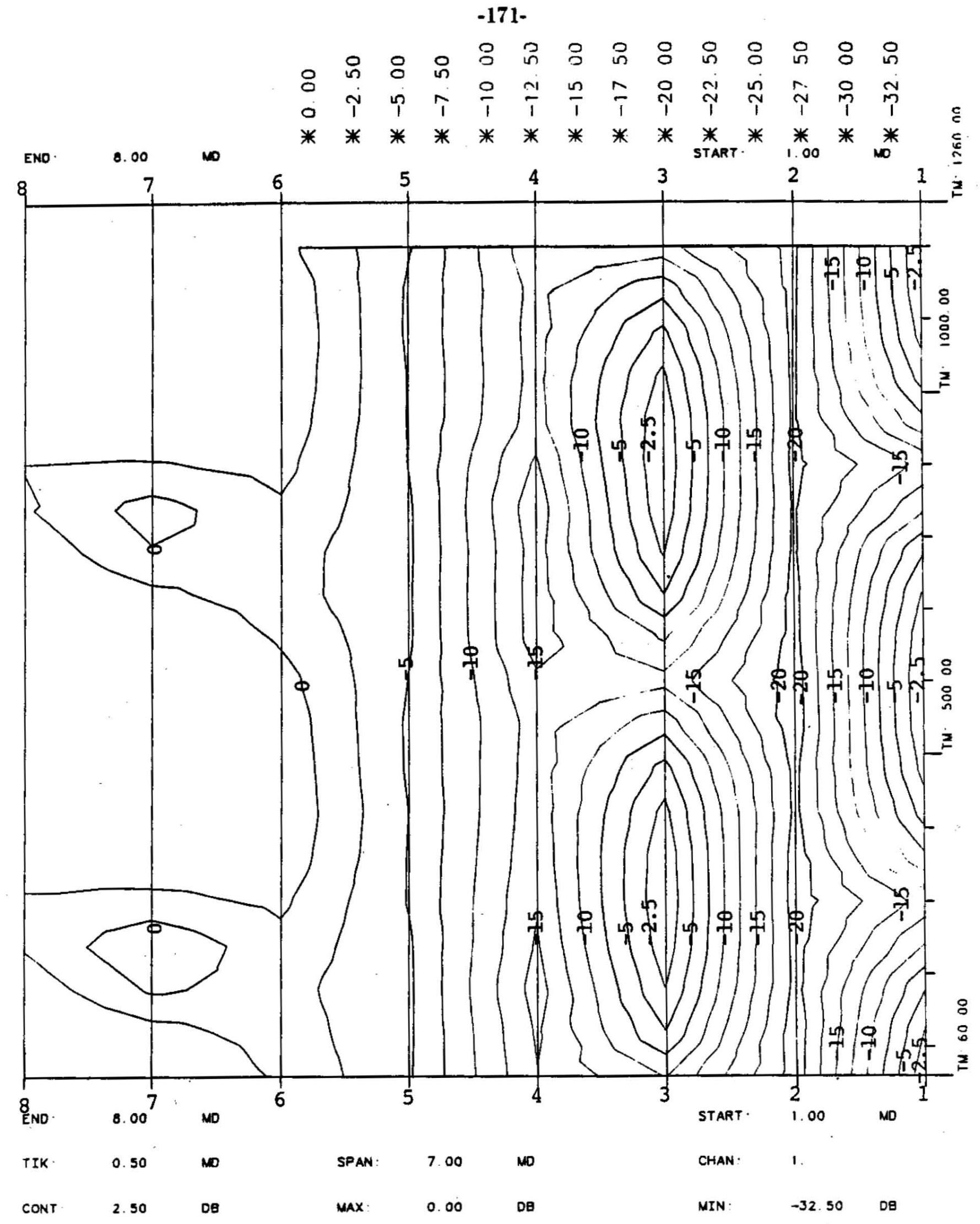

$47 \mathrm{HZ}$ - MULTIPLE BEAM LEAST SQUARES BEAMFORMER - COHERENT SYNTHETIC DATA

Figure 5-12: Output of Multiple Beam Least Squares Beamformer versus Time for Coherent Synthetic Data at $4 \pi \mathrm{Hz}$

( 8 modes included in beamformer) 


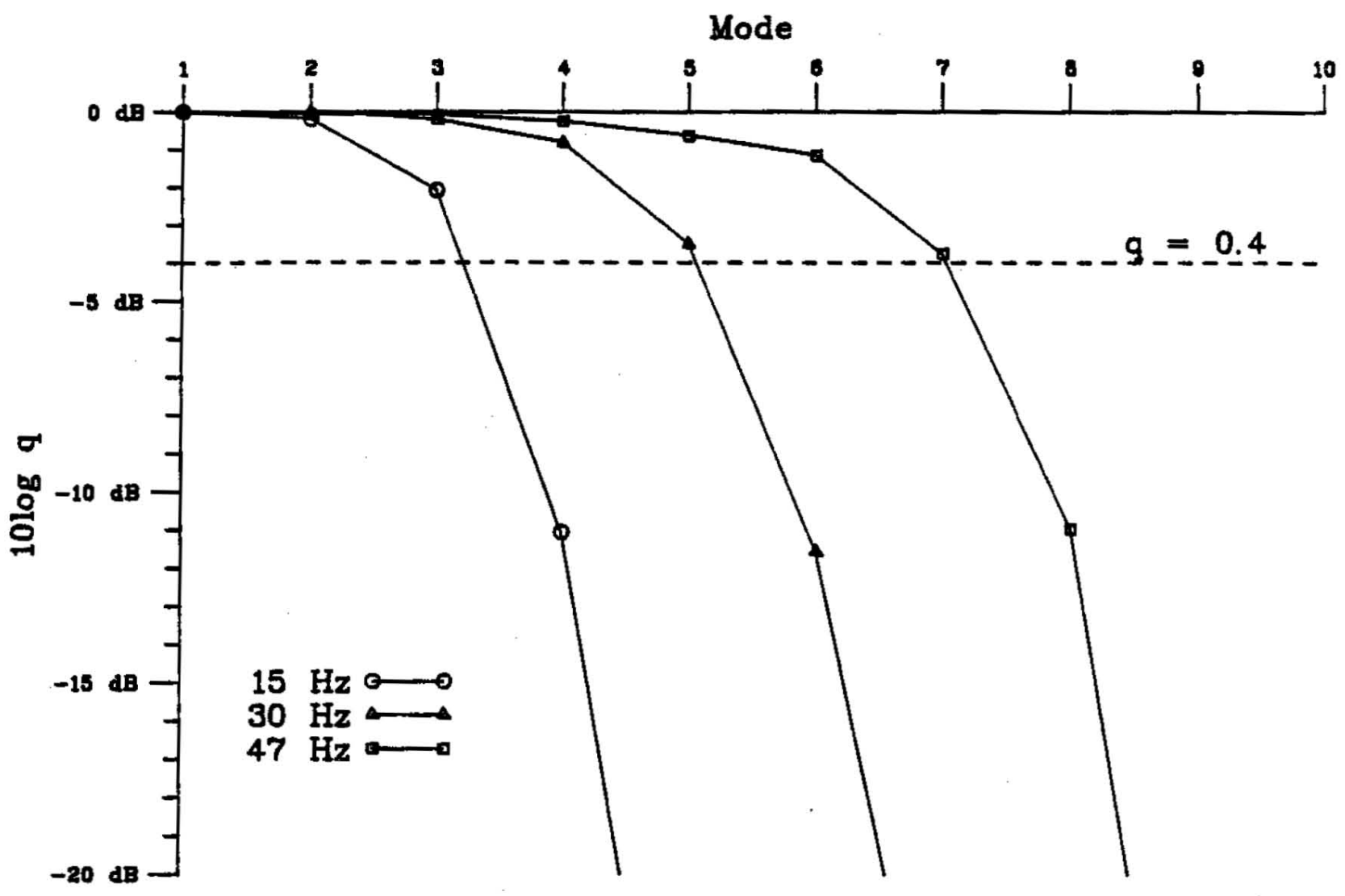

Figure 5-13: Singularity Coefficient versus Mode Count at $15 \mathrm{~Hz}, 30 \mathrm{~Hz}$, and $47 \mathrm{~Hz}$

between the increased resolution and the decreased numerical stability caused by the inclusion of additional modes.

The relative singularity of the above matrix can be quantified through the use' of the singularity coefficient $q$, which is defined as

$$
q=\frac{\operatorname{Det}\left[\overline{\mathbf{E}}^{+} \overline{\mathbf{E}}\right]}{\prod_{i=1}^{M}\left|\underline{\mathbf{E}}_{i}\right|^{2}}
$$

Here, the Det $[\cdot]$ notation is used to indicate the determinant of the matrix, while 
$\underline{\mathbf{E}}_{1}$ through $\underline{\mathbf{E}}_{M}$ are the various column vectors comprising the steering matrix $\underline{\overline{\mathbf{E}}}$.

The denominator consists of the product of the norms of these vectors.

The singularity coefficient has several very useful properties. First, it is always unity when $M=1$. Second, for any other value of $M$, it is unity only if all the component column vectors are all mutually orthogonal. Finally, its value is always in the range from 0.0 to 1.0. Since the coefficient is proportional to the value of the matrix determinant, it can approach zero only as the matrix becones singular. Thus, it provides a good indication of the relative singularity of the matrix $\underline{\mathbf{E}}^{+} \underline{\mathbf{E}}$.

Figure 5-13 provides a dB scale plot of this singularity coefficient for steering matrices consisting of up to the first nine modes at frequencies of $15 \mathrm{~Hz}, 30 \mathrm{~Hz}$, and $47 \mathrm{~Hz}$. In general, the coefficient remains close to 1.0 when small numbers of modes are included in the steering matrix, gradually decreasing as the mode count increases. Beyond a certain critical number of modes, though, inclusion of further modes in the steering matrix then causes the coefficient to fall rapidly towards zero. Various methods may be used to define precisely this critical number of moden. Here, a trial and error procedure has been used to find a reasonable breakpoint. The singularity coefficient has been compared against the processing output for synthetic data similar to that found in Figures 5-10 and 5-12 at various combinations of frequency and mode count. This comparison shows that optimum beamformer performance occurs for values of the singularity coefficient that are slightly greater than 0.4 . The critical mode count is then the largest numbrr of modes that can be included in the steering matrix without the coefficient exceeding this threshold.

This definition produces optimal results at all the frequencies tested in the trial and error procedure. However, it may be influenced by the array 
configuration, the modal structure of the particular channel investigated, and the choice of modes included in the synthetic data. The sensitivity of the definition to these various effects has not been investigated.

Using the above definition, the number of modes that can be included in the multiple beam algorithm at any frequency is readily found. This information is presented in Table 5-I for the frequencies available in the FRAM IV vertical array data set. Since the multiple beam processor computes the output of all its beams simultaneously, the value also represents the number of modes that can be resolved at each frequency. In all cases, at least three modes are resolvable; the number increases with frequency, reaching a value of 9 modes at $71 \mathrm{~Hz}$. A comparison with the mode shapes of Chapter 4 reveals that the last resolvable mode is the one in which the lowest oscillation of the mode shape is still sampled by at least one sensor.

\begin{tabular}{lc} 
Frequency & Mode Count \\
\cline { 2 - 2 } $15.00 \mathrm{~Hz}$ & 3 \\
$17.75 \mathrm{~Hz}$ & 3 \\
$20.00 \mathrm{~Hz}$ & 3 \\
$23.50 \mathrm{~Hz}$ & 4 \\
$30.00 \mathrm{~Hz}$ & 5 \\
$35.25 \mathrm{~Hz}$ & 5 \\
$35.50 \mathrm{~Hz}$ & 5 \\
$47.00 \mathrm{~Hz}$ & 7 \\
$53.25 \mathrm{~Hz}$ & 7 \\
$55.00 \mathrm{~Hz}$ & 7 \\
$71.00 \mathrm{~Hz}$ & 9
\end{tabular}

Table 5-I: Modes Resolvable by Multiple Beam Least Squares Modal Beamformer 


\subsection{Summary}

Several different methods for the decomposition into its modal components of the sound field observed at an array have been presented and analyzed. Modal decomposition is intimately related to the problem of beamforming, which has a rich history in the literature. The primary theoretical difference between the two results is the choice of the basis set of functions on which the received field is decomposed: traditional beamforming utilizes the set of plane waves, while the present effort employs the normal mode functions associated with the sound channel in which the receiving array resides. When the two approaches are compared, the major advantages of plane wave beamforming are the independence of the technique from knowledge of the sound channel and its equivalence with Fourier transform techniques, which allows a wide body of knowledge to also be tapped. The principal advantages of modal beamforming include the mathematically efficient nature of the representation and the physical relevance of the resultant outputs.

Two different approaches to modal beamforming have been addressed: the least squares method and the MLM algorithm. The two are differentiated by their choice of weighting matrix used in computing the total square error to be minimized. The least squares approach weights all errors equally, attempting to match the full received field. The MLM algorithm, on the other hand, tries to compensate for noise by selecting the inverse of the sensor cross-coherence matrix as the weighting matrix. This selection has the effect of weighting noisy sensors less heavily than sensors with clean signals for purposes of calculating the total square error. Both methods can be implemented in either a single or multiple beam format, generating four different approaches, all of which have been considered here. 
As might be expected, the resulting solutions for these different approaches to modal beamforming all take on the forms identical to their plane wave processing algorithm analogs. The only difference occurs in the definition of the steering matrix, which is the parameter describing the signal to be detected. In plane wave beamforming, this steering matrix (or steering vector, when dealing with single beam variants) consists solely of the complex phase accumulated in the travel of the plane wave from the receiver reference point to the individual sensor. For modal beamforming, the direction of travel is restricted to the horizontal plane, but the size of the mode shape at each sensor depth is included in addition to the phise accumulation due to travel.

The relative performance of the each of the four variants proposed for modal beamforming has been investigated for the case of the FRAM IV vertical array when deployed in the sound channel described in Chapter 4. The single beam least squares beamformer, which is the equivalent of the conventional beamformer, has been shown to exhibit inadequate modal resolution, being able to resolve only a single mode at $15 \mathrm{~Hz}$, and no more than 8 modes even at $71 \mathrm{~Hz}$. At intermediate frequencies, typical resolution is 3 modes at $30 \mathrm{~Hz}$ and 5 modes at $47 \mathrm{~Hz}$. In all cases, the number of modes that can be resolved is but a small percentage of those that might possibly provide significant contributions to the received sound field. The resolution has been shown to be limited by the length of the array rather than by sensor spacing within the array aperture.

The single beam MLM beamformer can provide significantly better resolution than the single beam least squares beamformer if the received sound field is comprised of modes which sum incoherently. It has also been demonstrated theoretically that the single beam variant of the MLM algorithm always exhibits better performance than the multiple beam variant under these conditions. Thus, 
the single beam MLM algorithm is the method of choice for accomplishing modal decomposition with the FRAM IV vertical array if the modes at the receiver combine incoherently.

The performance of the MLM algorithm has been shown to be very sensitive to the assumption of mode incoherence. The single beam variant of the algorithm operates only very poorly in the presence of coherently summed modes. This problem has been examined in detail, both theoretically and through simulation: the cause has been shown to be a violation of the assumed independence of signal and noise. This violation is produced because one of the coherent modes is implicit! classified as the signal and the others as noise in the single beam MLM variant. It has been shown that this problem can be triggered by the presence of even small amounts of a second coherent mode, and is most severe for the case of orthogonal steering vectors, as is the case for modes well resolved by a vertical array. The effect has been demonstrated to be less significant when the steering vectors of the coherently summing modes are approximately parallel to each other, such as occurs when considering high resolution horizontal array beamforming. Finally, it can be demonstrated that, in the presence of coherent modes, the single beam MLM beamformer still provides higher resolution than the single beam least squares beamformer when applied to the horizontal array. In this case, the peak locations normally remain accurate; the resultant output levels are not reliable, though.

The multiple beam variant of the MLM algorithm is theoretically capable of eliminating the difficulties encountered by the single beam in processing a field consisting of coherent modes. This is accomplished only at the expense of some reduction in performance, and is implemented by simultaneously including in the multiple beam MLM beamformer all modes with significant coherent contributions. However, a practical investigation indicates that the FRAM IV vertical array lacks 
the fundamental resolution necessary to guarantee the numerical stability of such an approach. Thus, neither variant of the MLM algorithm is considered to be adequate for the reduction of FRAM IV vertical array data, since (as is shown in Chapter i) the modal contributions turn out to be highly coherent.

The multiple beam least squares algorithm has been shown to be an adequate high resolution alternative to the single beam least squares algorithm for vertical array data, because it is insensitive to the issue of mode coherence, and because it provides the multiple mode resolution at lower frequencies that is lacking in the single beam variant. The major issue involved in implementing any such algorithm is the selection of the number of modes to model (or, equivalently, the number of modal beams to estimate simultaneously). Inclusion of too many modes that are fundamentally unresolvable by the array causes numerical stability problems in the matrix inversions required to implement the processing. A quantitative method for selecting the number of modes to be include in the multiple beam least squares beamformer is presented. This method is based on the relative singularity of the steering matrix when different numbers of modes are included. Using the method, which has been verified through simulation, Table 5-I has been compiled; it indicates the number of modes that can be simultaneously resolved at different frequencies when the multiple beam least squares processor is applied to data taken from the FRAM IV vertical array. The results of Table 5-I indicate that multiple modes can be resolved for all frequencies of interest. 


\section{Chapter 6}

\section{The Array Tilt Problem}

The signal processing aspects of modal beamforming have been discussed in the previous chapter. For the purposes of that discussion, the array has been assumed to be ideal in all respects. In particular, the locations of the sensors that comprised the array have been assumed to be known exactly. In practice, such sensor positions are rarely known precisely; this is certainly true for the FRAM N" arrays, as is discussed in Sections 3.5 and 3.7. The purpose of the present chapter is to investigate the sensitivity of the modal decomposition process to the types of sensor position errors likely to be encountered in practice.

The chapter begins with the selection of the linear tilt model for the shape of the FRAM IV vertical array. The model is very convenient, since only one parameter, the effective array tilt angle, is needed to completely specify it, even when the three-dimensional aspects of the problem are considered. Such an approach can be justified in two ways. First, it accounts for the majority of the sensor offsets found in the notional shapes presented in Section 3. $\boldsymbol{6}$, especially at larger tilt angles, where both the size and the effect of sensor position errors are greatest. Second, the physics of the problem are consistent with the conjecture that higher order array shape variations have less of an impact on the modal decomposition process than does the linear tilt.

Next, the tilt problem is studied so that one can qualitatively understand its effect on vertical array modal beamforming. This study is supported by results generated from synthetic data which depict graphically the tilt angle sensitivity of modal beamformers. The physical insight generated also leads to a simple method 
for estimating the accuracy of tilt angle measurements needed for valid modal separation to be guaranteed. This method is then applied to the FRAM IV vertical array and sound channel, and the results are both reported and compared with the synthetically generated outputs.

The discussion just outlined contributes in three ways. First, it emphasizes the general importance of vertical array tilt in the modal decomposition problem. Second, it provides at least a rudimentary method of estimating the quality of tilt angle measurements that are needed. Finally, it proves that the array tilt problem must be addressed for successful application of modal beamforming to the FRAM NV vertical array data. However, no tilt angle measurements are directly available from the experimental data package. In light of this situation, one must then attempt to recover estimates of the effective array tilt from the acoustic data.

The remainder of the chapter presents a simple method for deducing the effective array tilt from the acoustic data set. This method involves the minimization of the residual total square error over a range of reasonable tilt angles. The residual total square error is a measure of how well the sound pressure field observed at the various array sensors is matched by a pressure field reconstructed from the complex modal amplitude outputs of the beamformer. The accuracy of the method is investigated with synthetic data, and some conclusions are drawn about the conditions required to obtain reliable results.

\subsection{A Simplified Model for the Shape of the Vertical Array}

Two estimates of the true shape of the vertical array in the presence of reasonably severe relative currents have been presented in Section 3.7. The methods used to generate these estimates can be considered mathematical models of the vertical array shape. Both of the models are relatively crude, even to the point 
of being somewhat unrealistic. From a signal processing point of view, though. still further simplification is desirable, so that the number of parameters needed to define the shape of the array can be kept to a minimum. In the remainder of this thesis, the array is modeled as a simple straight line that makes some angle with the true vertical. In terms of the signal processing, this is the simplest model possible, since it requires only one parameter, the effective array tilt angle, to completely determine the model array shape.

The selection of the simple linear tilt model for the vertical array shape can be justified by examining how well it fits the actual shape of the array under different conditions. Since no actual array shape data is available, this is difficult to do directly. Instead, the comparison is made with the NRL array model on the assumption that the model is at least a reasonable reproduction of the general shape of the array. The fit can be quantified by breaking the NRL result presented in Figure 3-5 into polynomials involving increasing powers of $z$. The natural basis set for such a decomposition is the set of normalized Legendre polynomials, since they are the orthogonal function set involving increasing powers in $z$. The coefficient of the first order term in the expansion represents the assumed tilt angle that the array makes with the vertical, while those of higher terms indicate the relative contributions that more complex curves make to the total array shape. The more commonly used Taylor series expansion is misleading in this situation, since the Taylor polynomials are not orthogonal. The coefficient of the first order Taylor term does not reflect the full tilt of the array, since odd Taylor polynomials of higher order $\left(z^{3}, z^{5}\right.$, etc.) all contain additional implied array tilt.

From orthogonal function theory [20], the coefficient of the $i^{\text {th }}$ term of the series can be computed as

$$
a_{i}=\sqrt{\frac{H}{2}} \int_{-1}^{1} P_{i}\left(z^{\prime}\right) r^{\prime}\left(z^{\prime}\right) d z^{\prime},
$$


where $z^{\prime}$ is a normalized depth coordinate in the range from -1 to 1 , being related to the unnormalized depth $z$ and the total length of the array $H$ by

$$
z^{\prime}=\left(\frac{2}{H}\right) z-1 \text {. }
$$

The notation $r^{\prime}\left(z^{\prime}\right)$ is used to indicate the actual array offset as measured at the equivalent unnormalized depth. $P_{i}\left(z^{\prime}\right)$ is the $i^{\text {th }}$ Legendre polynomial; the lowest order functions of this set are provided in Table 6-I. In addition, the same theory guarantees that

$$
I=\frac{H}{2} \int_{-1}^{1} r^{2}\left(z^{\prime}\right) d z^{\prime}=\sum_{i=0}^{\infty} a_{i}^{2},
$$

so that the percentage contribution of any term to the full array shape can be computed by dividing the square of the appropriate coefficient by $I$.

Table 6-I provides the results of the Legendre polynomial decomposition for the first few terms of the series. It is easy to see that the series can be reasonably well approximated with as few as the first two terms. Since the zero order term represents nothing more than a translation of the horizontal axis, it has no practical effect on the beamforming, and can be completely eliminated by proper redefinition of the coordinate system. However, even if the zero order term is ignored, the first order linear tilt term still accounts for about $90 \%$ of the remaining array offset.

Thus, the conclusion is reached that the array shape displayed in Figure 3-5 can be adequately represented by an array that remains a straight line but which is tilted at some angle to the vertical. Whether or not the array can normally be represented by a linear tilt is more problematic. A quantitative discussion of this point is clearly impossible, due to the lack of experimental array shape measurements. However, two general arguments can be made in favor of such a 


$\begin{array}{lccc}\underline{\text { Polynomial }} & \frac{\text { Coefficient }\left(\mathrm{m}^{3 / 2}\right)}{\underline{\text { with }}_{0}} & \begin{array}{l}\text { Relative Contribution } \\ \text { without } a_{0}\end{array} \\ P_{0}\left(z^{\prime}\right)=\sqrt{0.5} & 9.82 \times 10^{2} & 80.02 \% & - \\ P_{1}\left(z^{\prime}\right)=\sqrt{1.5} z^{\prime} & 4.62 \times 10^{2} & 17.72 \% & 88.68 \% \\ P_{2}\left(z^{\prime}\right)=\sqrt{2.5}\left(1.5 z^{\prime 2}-0.5\right) & -1.65 \times 10^{2} & 2.26 \% & 11.32 \% \\ P_{3}\left(z^{\prime}\right)=\sqrt{3.5}\left(2.5 z^{\prime 3}-1.5 z^{\prime}\right) & 3.35 \times 10^{0} & - & - \\ & & \\ I=1.205 \times 10^{6} \mathrm{~m}^{3}\end{array}$

Table 6-I: Results of Legendre Polynomial Analysis Results for the NRL Model of the FRAM IV Vertical Array Shape

conclusion. First, the structural mechanics of the vertical array suggests that the lowest order shape components should dominate. For the length and weight involved (1000 $\mathrm{m}$ and roughly 2 tons), it is difficult to visualize a condition where much more than a very few low order terms of the expansion contribute significantly. This supposition agrees with the results presented in Table 6-I, where anything beyond the parabolic term is obviously negligible. Second, it must be realized that the accuracy of the linear tilt model can be expected to generally increase at higher currents, where the array shape effects are larger and their impact on the problem is more significant. As the array becomes strung out at these currents, the proportion of the array shape provided by the tilt term increases. Thus, the model is most accurate in the worst case condition, where it is needed most. Finally, it is possible to argue that it is necessary to retain the second order term for accuracy, but such a decision must be balanced against the increased complexity of the resulting model. In the present case, the parabolic term appears 
to be small enough that more is gained by dropping it than by retaining it.

The true array shape is a three-dimensional function, as shown in Figure 3-5. However, it is only one projection of the full shape (that in the vertical plane of sound propagation) that affects the modal beamforming. For the purposes of the Legendre polynomial analysis just conducted, this vertical plane was arbitrarily assumed to be coincident with the vertical plane of current flow at the surface, which will not generally be the case. In a more general situation, the linear tilt model can be thought of as the first order Legendre polynomial fit to the projection of the array shape into the vertical plane of propagation. The tilt angle associated with this linear tilt is then not a true array tilt, but only an effective one measured in the direction of propagation.

\subsection{The Sensitivity of Modal Beamforming to Array Tilt}

Having demonstrated that the first order effect of sensor offsets in vertical arrays is to provide an effective tilt to the array, the sensitivity of the modal decomposition process to this tilt must next be considered. Consider the simple channel shown in Figure 6-1, which consists of a free surface at $z=0$, a hard bottom at a depth of $z=H$, and a sound speed $c_{0}$ that is constant throughout the channel. For simplicity, assume an array of continuous aperture that extends to the full depth of the channel, and a situation where only the $i^{\text {th }}$ mode is present. For such a case, the multiple beam least squares result of Chapter 5 reduces to the modal equivalent of the conventional beamformer. The resulting processor may also be interpreted as a traditional matched filter, but in a spatial sense. Thus, to detect the $i^{\text {th }}$ mode, the output of the array at any depth should be weighted by the size of the mode shape at that depth, which for this channel is 


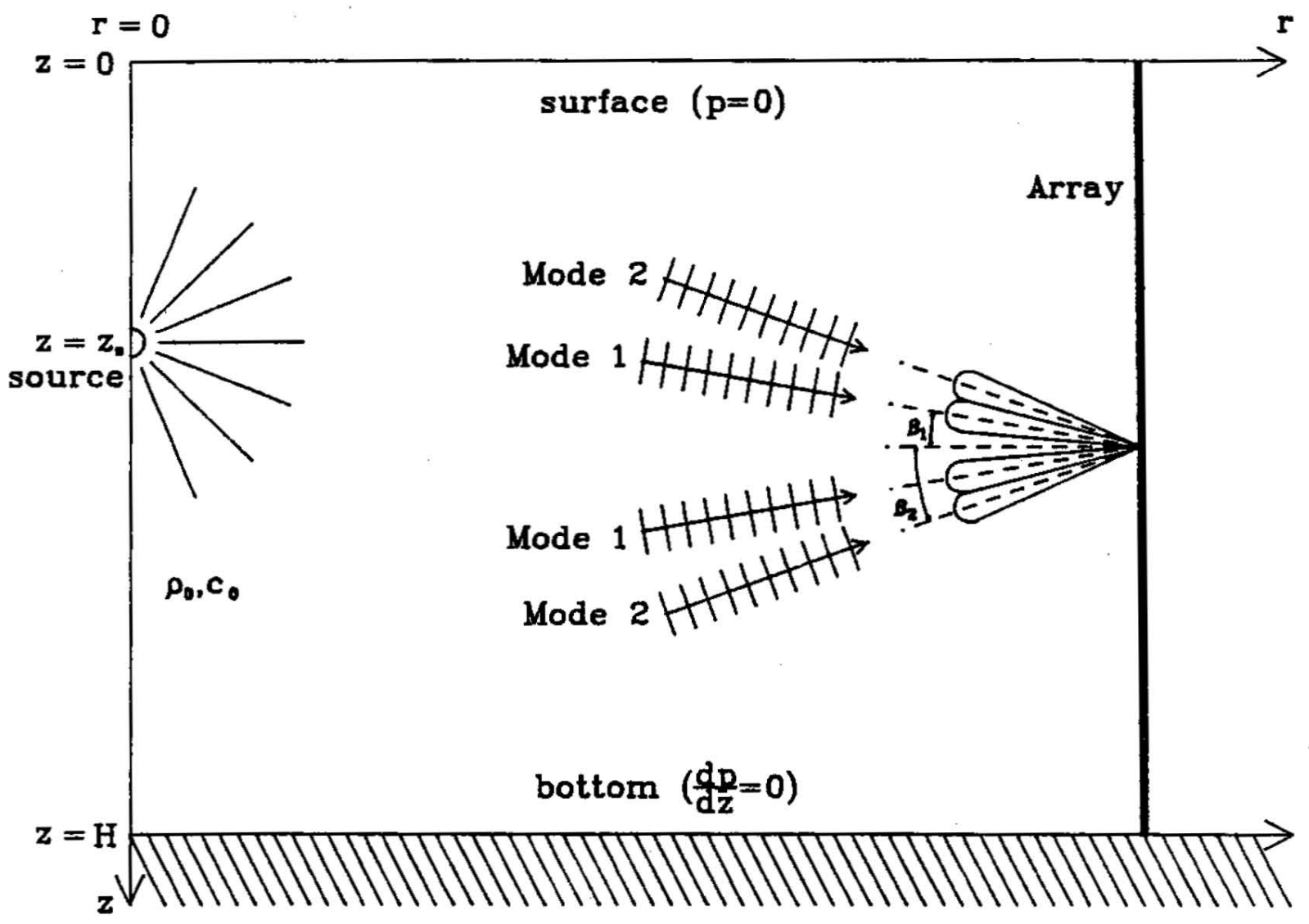

Figure 6-1: Modal Beamforming for an Untilted Array in a Simple Channel

$$
\phi_{i}(z)=\sqrt{\frac{2}{H}} \sin \left(\nu_{i} z\right)=\frac{1}{j \sqrt{2 H}}\left(e^{j \nu_{i} z}-e^{-j \nu_{i} z}\right) .
$$

Here $v_{i}$ is the vertical wavenumber of the mode, given by

$$
\nu_{i}=\frac{(i-1 / 2) \pi}{H} .
$$

The second expression in equation (6.4) provides another interpretation of modal beamforming. The weighting of the array aperture by the mode shape is 


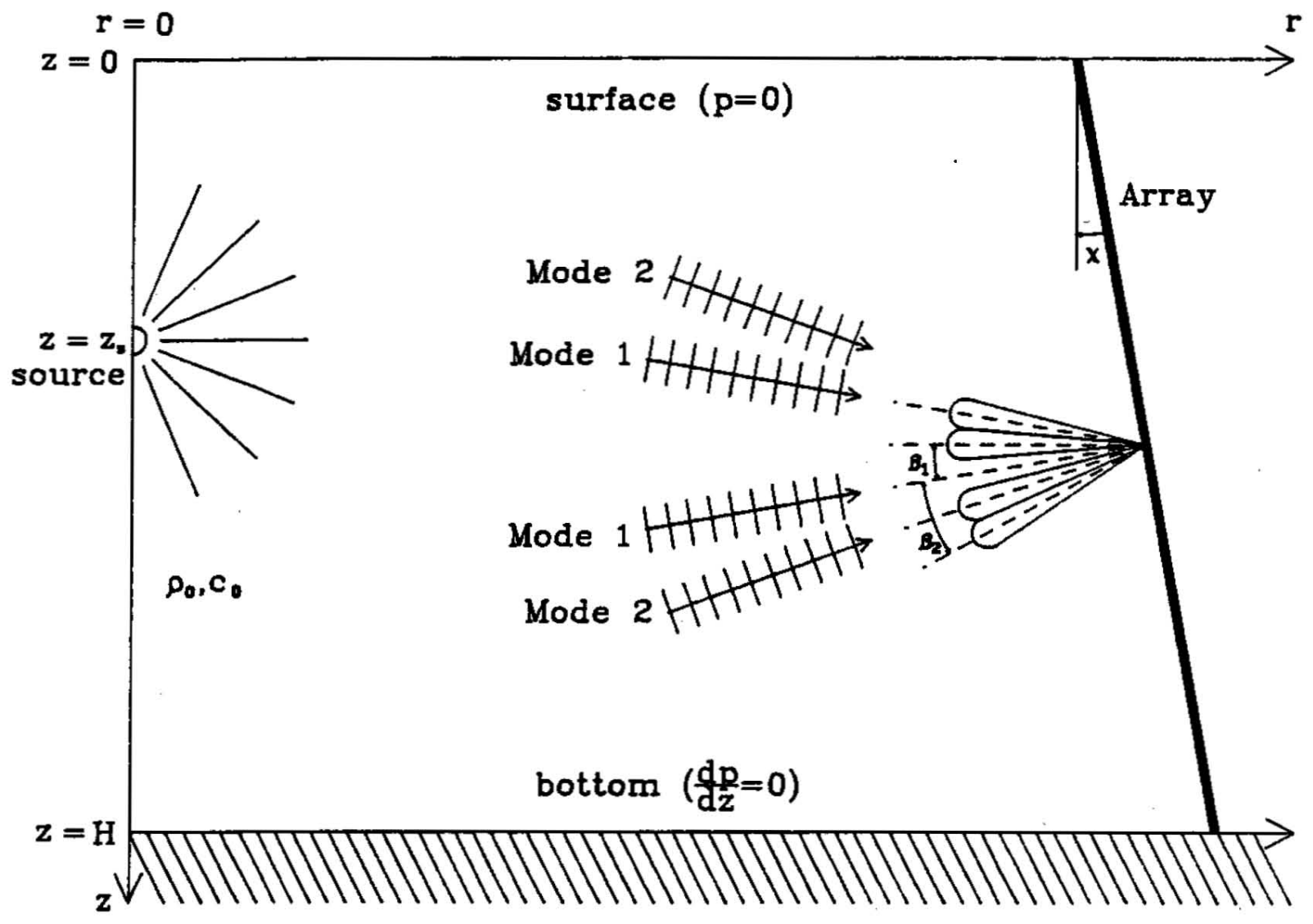

Figure 6-2: Modal Beamforming for a Tilted Array in a Simple Channel

equivalent to simultaneously forming two plane wave beams, one at angle,$_{i}$ to the horizontal and the other at angle $-\beta_{i}$, where

$$
\beta_{i}=\sin ^{-1}\left(\frac{\nu_{i} c_{0}}{2 \pi f_{0}}\right)=\cos ^{-1}\left(\frac{c_{0}}{c_{i}}\right) \text {. }
$$

The outputs of these two beams are then subtracted and properly scaled to form the modal amplitude estimate. In equation (6.6) $c_{i}$ is the horizontal phase speed associated with the $i^{\text {th }}$ mode, which is related to the vertical wavenumber by

$$
c_{i}=\left[\left(\frac{1}{c_{0}}\right)^{2}-\left(\frac{\nu_{i}}{2 \pi f_{0}}\right)^{2}\right]^{-1 / 2} \text {. }
$$


This interpretation is not surprising, since the equivalence of each mode with a pair of plane waves is well established for this particular channel.

Because the modal beam is no more than the sum of a pair of plane wave beams for this case, the $i^{\text {th }}$ modal beam can be expected to respond to energ: traveling in either the upward or downward direction at a grazing angle of $x_{i}$ : further, this is true whether or not the energy is really being carried by the $i^{\text {th }}$ mode. Now, consider the effect of imposing a linear tilt on the array, which is illustrated in Figure 6-2. The tilt causes the plane wave beam pairs to be aimed away from the directions in which the modal energy is actually arriving. In fact. if the array tilts too much, then one or both of the equivalent plane wave beams can end up pointed in a direction where it detects the arriving energy of a neighboring mode instead of the energy carried by its own mode. Since it is obvious that the modal beamformer responds to this energy, one can readily anticipate severe distortions in the modal decomposition if the effective array tilt angle becomes too large.

It is of interest to consider the effect of higher order shape terms from a similar viewpoint. Whereas the linear tilt term tends to redirect the equivalent plane wave beams, the presence of higher order shape terms causes decreasing amounts of mis-steering in the beam formation and increasing amounts of beam defocusing. Thus, the linear tilt approximation is probably better suited to the array shape modeling problem than expected, since it retains the portion of the actual array shape that most significantly affects modal decomposition. 


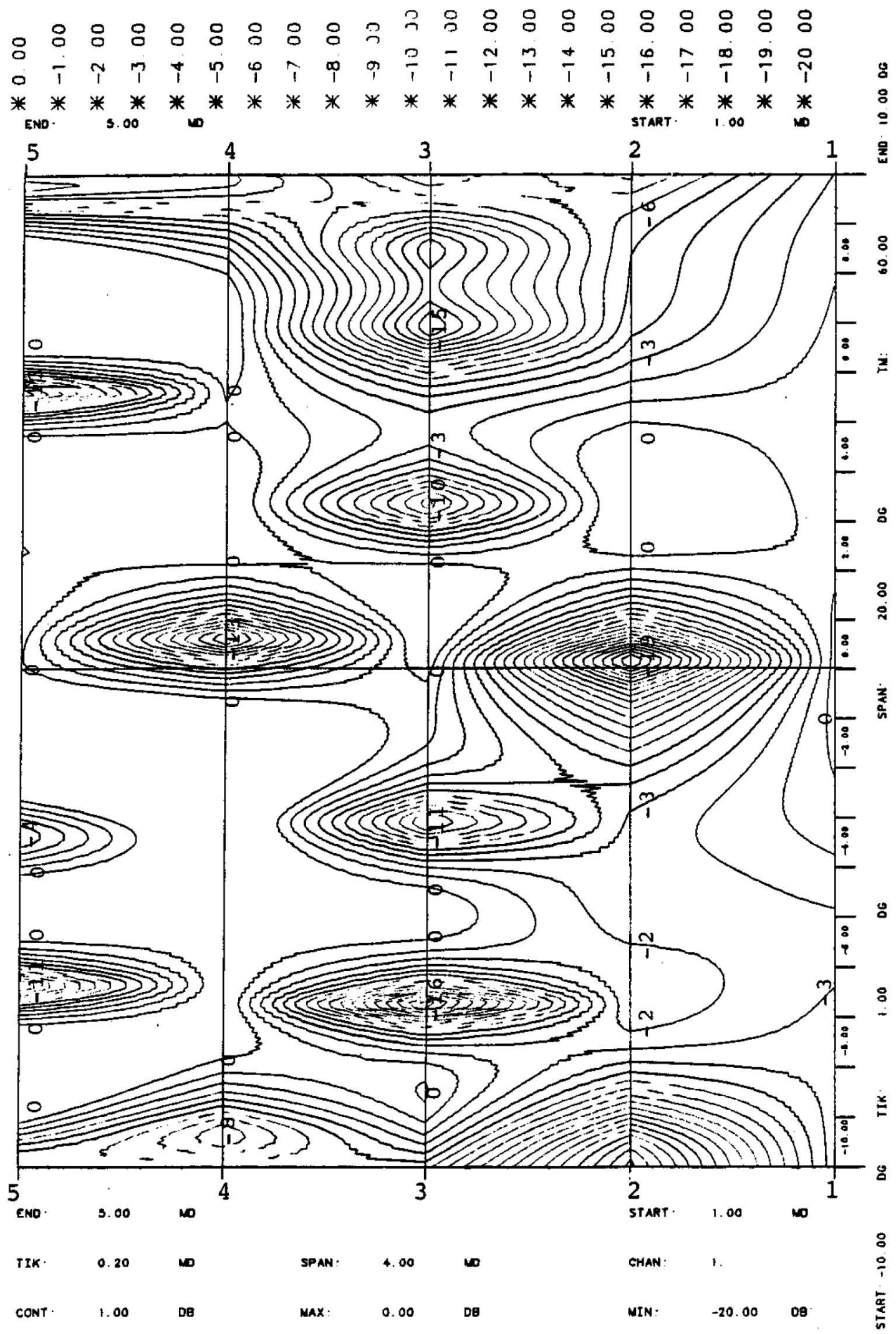

$30 \mathrm{HZ}$ - MULTIPLE GEAM LEAST SOUARES GEAMFORMER - CDHERENT SYNTHETIC DATA

Figure 6-3: Sensitivity of Vertical Array Modal Beamforming to Array Tilt (Synthetic Data at $30 \mathrm{~Hz}$ - First Realization) 


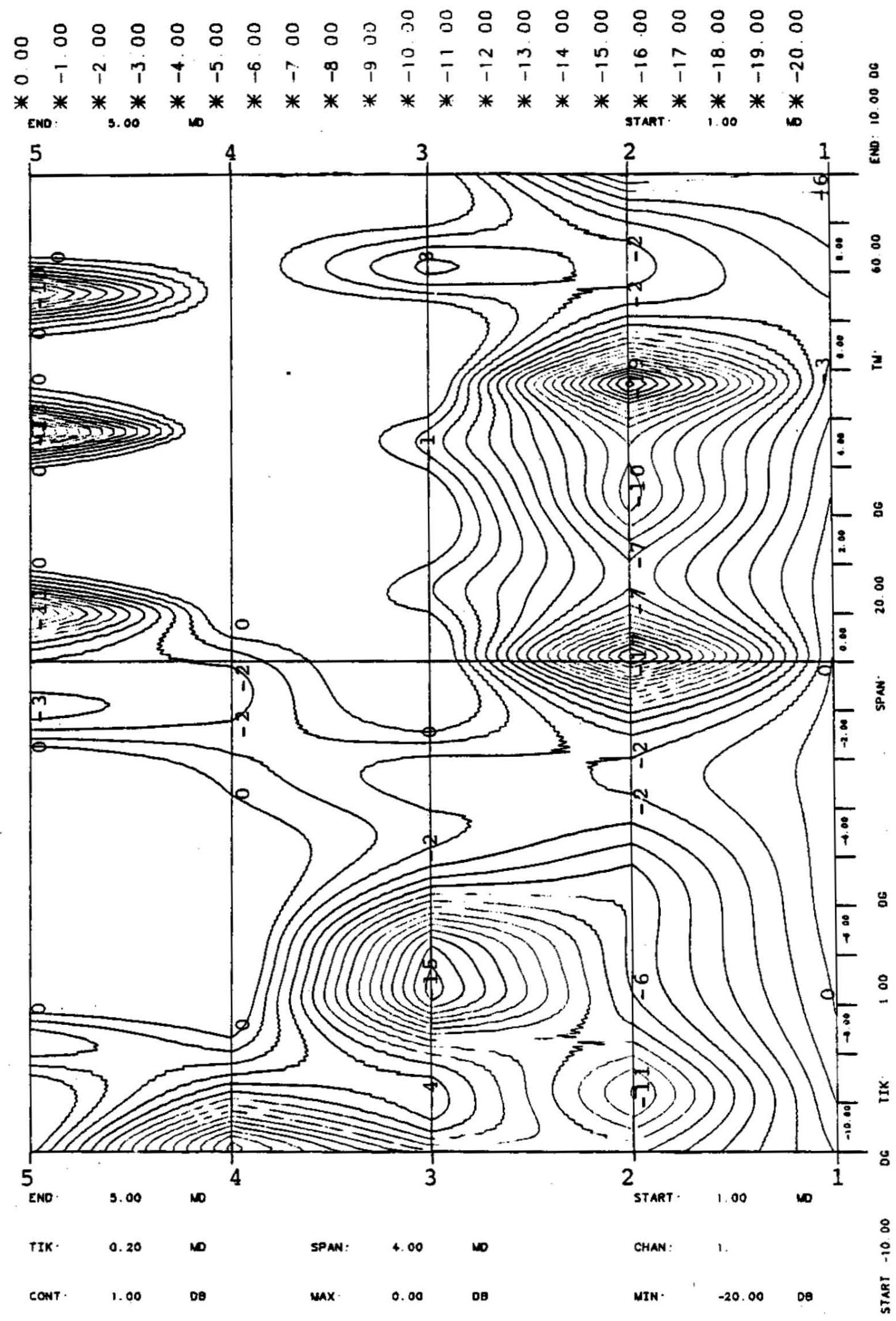

$30 \mathrm{HZ}$ - MULTIPLE BEAM LEAST SOUARES GEAMFORMER - COHERENT SYNTHETIC DATA

Figure 6-4: Sensitivity of Vertical Array Modal Beamforming to Array Tilt (Synthetic Data at $30 \mathrm{~Hz}$ - Second Realization) 


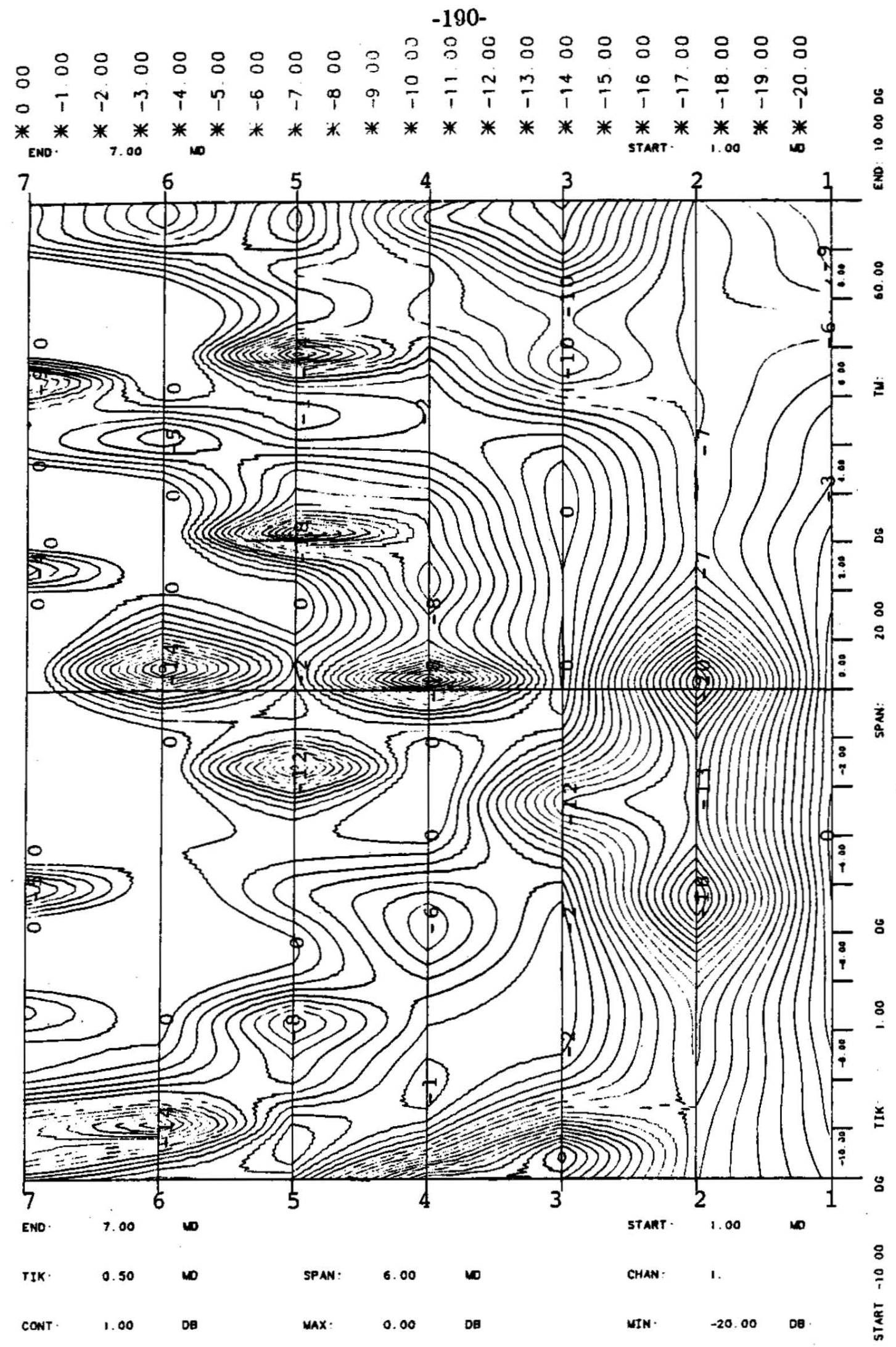

$47 \mathrm{HZ}$ - MULTIPLE GEAM LEAST SOUARES BEAMFORMER - COHERENT SYNTHETIC OATA

Figure 6-5: Sensitivity of Vertical Array Modal Beamforming to Array Tilt (Synthetic Data at $\mathbf{4} \mathbf{~ H z}$ ) 


\subsection{Examples of the Array Tilt Sensitivity in the Processing}

To highlight the sensitivity of modal beamforming to the assumed tilt angle, several synthetic data sets have been created and processed with the multiple beam least squares algorithm described in Chapter 5. The synthesized sound fields examined are similar to those used in the previous chapter, since each signal field has been generated by coherently summing contributions from the odd modes between mode 1 and mode 15. Each mode has been assigned a unit amplitude and a random initial phase. Unlike the synthetic data presented in Chapter 5, though, the signals analyzed here have been created with constant, unmodulated modal amplitudes. The array has been assumed to be precisely vertical for the full length of each synthetic signal. The multiple beam least squares algorithm used to accomplish the beamforming is identical in all respects to that employed in the previous chapter. The window length and processing interval selections are also the same.

Figures 6-3 and 6-4 illustrate two different examples of the results for synthetic data sets at $30 \mathrm{~Hz}$, while Figure 6-5 provides a similar example at $4 \mathbf{7} \mathrm{Hz}$. To develop these figures, the modal amplitudes for a single processing window of data have been computed at a series of different assumed vertical array tilt angles. The resultant modal amplitude outputs have then been plotted as a function of the effective tilt angle assumed in generating them. As in Chapter 5, the vertical axis represents the mode number to which the amplitude data applies. Since the mode number axis really ranges over a discrete set, actual amplitude data occurs only at the integer mode numbers, where the black horizontal lines have been drawn. The contours display the variation of amplitudes on the mode-tilt plane for one particular window of data. Results are provided for all 5 of the modes resolvable by the vertical array at $30 \mathrm{~Hz}$ and for the 7 modes that can be distinguished at $47 \mathrm{~Hz}$. 
The horizontal axis indicates the effective tilt angle assumed in the beamforming. Thus, the vertical column of data at $0^{\circ}$ indicates the true distribution of energy in the mode set (since the synthetic field is generated for a truly vertical array). On the other hand, the column of data at $1^{\circ}$ indicates the modal distribution generated by the beamformer if $1^{\circ}$ of mismatch is present between the actual tilt and the tilt assumed by the beamformer.

All three plots vividly illustrate the variation in modal beamformer outputs that can occur when even slightly different vertical array tilts are assumed. As expected, the $0^{\circ}$ outputs accurately reflect the actual modal distribution present in the synthetic field. It is easy to observe the expected alternating arrangement of deep nulls (at even modes) and strong peaks (at odd modes) at this tilt. Although one is unlikely to encounter the full range of assumed tilt angles $\left( \pm 10^{\circ}\right)$ in practice, it is not unreasonable to expect excursions of as much as $\pm 5^{\circ}$. The large variations of the modal amplitude estimates even within this smaller range of tilts show that knowledge of the actual tilt angle is necessary for valid modal decomposition.

The synthetic data sets used to generate Figures 6-3 and 6-4 differ in only one respect, that being the initial phase relationships assumed between the various modes making up the signal field. Both figures are included to demonstrate that the pattern of peaks and nulls generated is highly dependent on these phase relationships. A comparison between either of these figures and Figure 6-5 shows that, as might be expected, the processing becomes more sensitive to tilt angle at higher frequencies. Amplitude estimates for higher modes also exhibit greater sensitivity than those for lower modes at the same frequency. If one wishes to assure reasonable accuracy in first mode amplitude estimates, it appears that the array tilt must be known to roughly the nearest $1^{\circ}$. Estimates at higher modes require much more precise tilt data to be valid. 


\subsection{The Array Tilt Accuracy Required for Valid Modal Estimates}

Given the sensitivity of the vertical array processing to assumed array tilt, estimation of the accuracy required in the tilt angle measurement to guarantee valid modal decomposition becomes important. One simple approach to this problem is outlined here. From Figure 6-2, it is obvious that, for a simple uniform channel, the modal separation is certainly invalid by the time the array has been tilted far enough to cause one of the pair of equivalent plane wave beams to point in the direction of a neighboring mode's arriving energy. Adopting a criterion for valid results of one half of this tilt leads to the expression

$$
\chi_{\text {max }}=\frac{1}{2} \operatorname{MIN}_{i}\left[\cos ^{-1}\left(\frac{c_{0}}{c_{i+1}}\right)-\cos ^{-1}\left(\frac{c_{0}}{c_{i}}\right)\right],
$$

where the second half of equation (6.6) has been used to evaluate each of the grazing angles. The minimization must be conducted over all modes to be properly estimated.

Equation (6.8) is strictly correct only for a hard bottom, constant sound speed channel, where the equivalence of the mode to a pair of plane waves is exact. Applying it to more general channels requires further justification. Typically, each mode is equivalent to a full spectrum of plane waves across vertical wavenumber space, rather than just the discrete pair considered here. This spectrum can be calculated by taking the Fourier transform in depth of the mode shape. However. mode shapes often exhibit a sort of characteristic vertical wavenumber, as can readily be observed by studying Figures 4-6 and 4-7. In such a case, the resulting wavenumber spectrum can be expected to show a sharp peak neir this characteristic value. It is not unreasonable to approximate such a spectrum by a pair of delta functions, which then completes the identification with an equivalent plane wave pair. Yang and Giellis [84] provide several examples of modal spectra 
that confirm the presence of these strong wavenumber peaks in the central Arctic mode set.

There remains the issue converting the characteristic vertical wavenumber value for each mode into an equivalent grazing angle. This is trivial for a channel of uniform sound speed, since it is easy to define a horizontal reference; any mode propagating in exactly the horizontal direction must possess a horizontal phase speed of $c_{0}$. The choice of a proper definition for the horizontal reference in a channel of arbitrary sound speed profile is much more subtle. Care in this matter is important, since the form of equation (6.8) makes it sensitive to the choice of $c_{0}$.

The problem is often dealt with by making a "relative" conversion; that is, the difference in grazing angle between two modes is computed by arbitrarily assigning the role of $c_{0}$ to the mode having the lower horizontal phase speed. A simple numerical example is enough to demonstrate the flawed nature of such an approach. Assume that the phase speed of the first mode is $1450 \mathrm{~m} / \mathrm{sec}$ at a given frequency, while that of the second mode is $1455 \mathrm{~m} / \mathrm{sec}$. Also assume that the reference phase speed associated with horizontal propagation is $1440 \mathrm{~m} / \mathrm{sec}$. Then the actual difference between the equivalent grazing angles of the two modes is $1.5^{\circ}$, whereas a value of $4.8^{\circ}$ is given by the relative conversion method. While this level of accuracy may be acceptable for problems to which the relative conversion is typically applied, it is obviously inadequate for the present purposes.

A definition of $c_{0}$ (the horizontal phase speed associated with propagation al a grazing angle of $0^{\circ}$ ) is needed that is general enough to be applicable to a channel having an arbitrary sound speed profile. Such a definition can be developed by identifying horizontal propagation with the mode having the smallest horizontal phase speed. The mode possessing this property is always the "DC'" mode. i.e., the lowest order mode for the desired channel when both rigid surface and rigid 


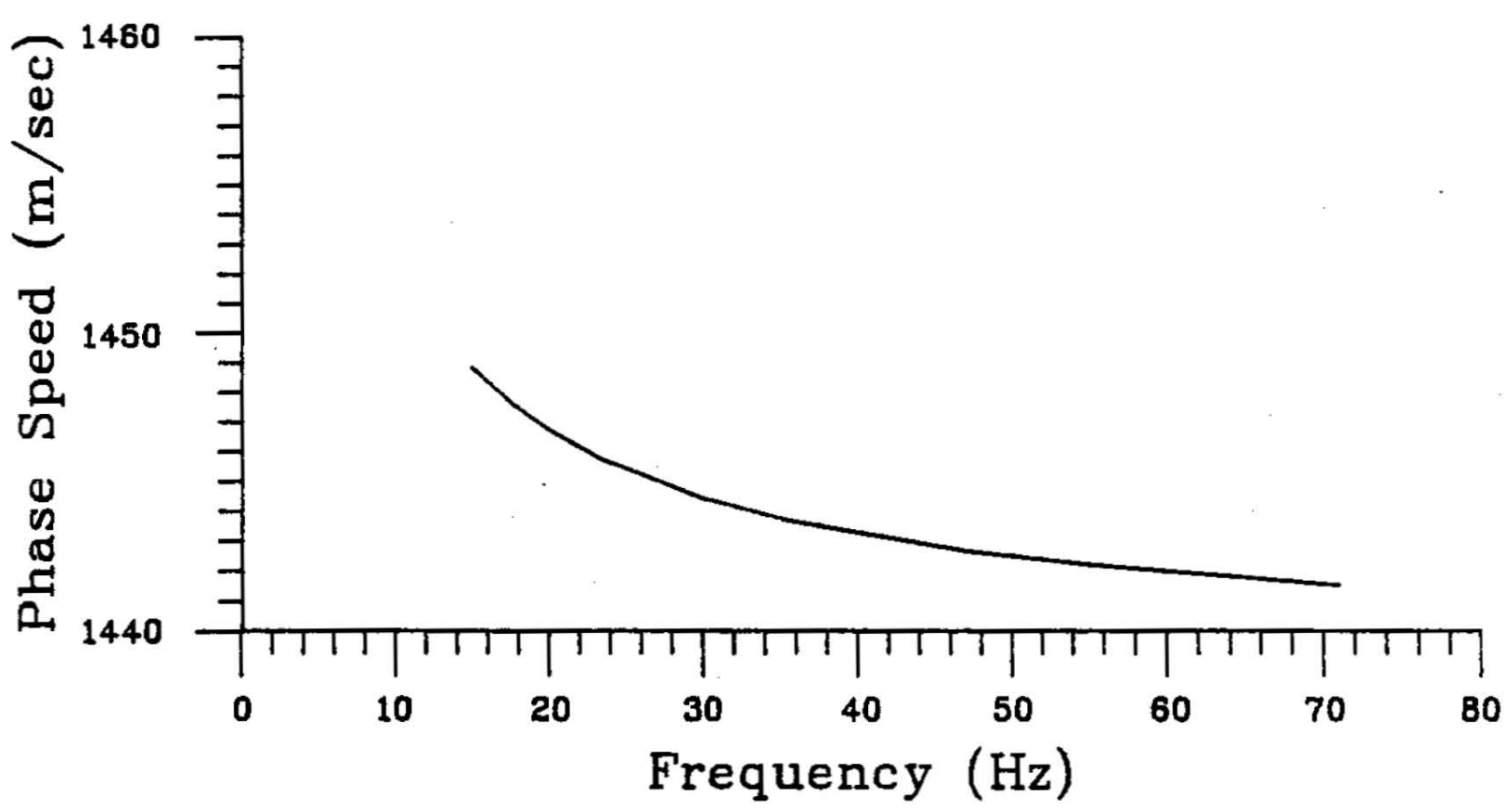

Figure 6-6: Horizontal Phase Speed for the DC Mode versus Frequency

bottom boundary conditions are assumed. It is named the DC mode here because the resulting mode shape is constant with depth for a channel of uniform sound speed, and, thus, represents the DC component of the resultant Fourier series. Note that this definition is consistent with the exact result derived above, since the phase speed of the DC mode for a channel of uniform sound speed is exactly $c_{0}$. Th" phase speed of this mode for the sound speed profile of Figure 4-1 is plotted versils frequency in Figure 6-6.

Table 6-II has been computed from equation (6.8) and Figure 6-6. It indicates the accuracy required in the array tilt measurements if valid modal amplitude estimates are to be assured for the modes resolvable in the FRAM $N$ vertical array data set. It can be seen that accuracies of better than $0.25^{\circ}$ are often neederl. These results agree well with the tilt angle sensitivities observed in Figurt's b-3 
through 6-5, where significant changes in the modal amplitude estimates can be observed over tilt changes of much less than $1^{\circ}$. The higher tolerance of the first. mode estimate to tilt angle variations that was noted earlier appears to be due to the greater separation in phase speed between it and the other modes; this causes an equivalent increase in the tilt angle needed to alias the resultant amplitude estimate. A similar phenomenon can begin to be seen for the second mode at higher frequencies. Note that the accuracy requirements generally increase at both higher frequencies and higher mode numbers. This is in keeping with the observations made from Figures 6-3 through 6-5.

\begin{tabular}{|c|c|c|c|c|}
\hline Frequency & 1 Mode & $2+$ Modes & & \\
\hline $15.00 \mathrm{~Hz}$ & $0.45^{\circ}$ & $0.40^{\circ}$ & & \\
\hline $17.75 \mathrm{~Hz}$ & $0.50^{\circ}$ & $0.35^{\circ}$ & & \\
\hline $20.00 \mathrm{~Hz}$ & $0.65^{\circ}$ & $0.30^{\circ}$ & & \\
\hline $23.50 \mathrm{~Hz}$ & $0.90^{\circ}$ & $0.25^{\circ}$ & & \\
\hline $30.00 \mathrm{~Hz}$ & $1.35^{\circ}$ & $0.20^{\circ}$ & & \\
\hline $35.25 \mathrm{~Hz}$ & $1.60^{\circ}$ & $0.15^{\circ}$ & & \\
\hline $35.50 \mathrm{~Hz}$ & $1.60^{\circ}$ & $0.15^{\circ}$ & & \\
\hline Frequency & 1 Mode & 2 Modes & $3+$ Modes & \\
\hline $47.00 \mathrm{~Hz}$ & $1.80^{\circ}$ & $0.35^{\circ}$ & $0.10^{\circ}$ & \\
\hline $53.25 \mathrm{~Hz}$ & $1.80^{\circ}$ & $0.50^{\circ}$ & $0.10^{\circ}$ & \\
\hline $55.00 \mathrm{~Hz}$ & $1.80^{\circ}$ & $0.55^{\circ}$ & $0.10^{\circ}$ & \\
\hline Frequency & 1 Mode & 2 Modes & 3 Modes & \pm+ Iodes \\
\hline $71.00 \mathrm{~Hz}$ & $1.60^{\circ}$ & $0.90^{\circ}$ & $0.20^{\circ}$ & 0.07 \\
\hline
\end{tabular}

Table 6-II: Tilt Angle Accuracy Requirements for the FRAM $\Pi$ Vertical Array

From Table 6-II, it can be seen that the tilt accuracies required to asiure proper modal decomposition of the received field are significantly smaller than the range of tilt angles likely to be encountered in the field. Thus, very accurate array tilt measurements must be made either directly or indirectly if a valid modal beamforming experiment is to be conducted. The FRAM IV vertical array tilt 
accuracy requirements are probably typical of those found elsewhere, while in other situations somewhat larger ranges of tilt angle will be encountered. Therefore. it is reasonable to conclude that vertical array tilt monitoring must be considered as an important integral element of any reliable modal decomposition scheme.

\subsection{The Effect of Tilt on Horizontal Arrays}

The sensitivity of the vertical array to array tilt is generated by a confluence of factors; these factors tend to work in just the opposite fashion for horizontal arrays, making them relatively insensitive to array tilt issues. The first order effect of ocean currents on a vertical array is the generation of a tilt across the face of the array. By comparison, the first order effect for a horizontal array is a uniform offset of sensors across the array, which has no influence on array performance for far field beamforming. Some variation of individual sensor offsets is experted. but this is generally small when compared to the mean offset. Such variations may cause some degradation in horizontal array beamformer performance at higher frequencies, where exact sensor position information is more critical. Even the fluctuations around the mean offset generate no real array tilt, since they tend to be randomly distributed across the array. Finally, because a horizontal array is essentially being operated at endfire when used for modal decomposition. the algorithm itself can be expected to be relatively insensitive to tilt. However, it should be realized that it is exactly this endfire operation that limits the modal resolution of horizontal arrays in the first place. In general, then, array tilt does not appear to be as significant an issue for the horizontal arrays as it is for vertical arrays. 


\subsection{Recovery of Vertical Array Tilts from the Acoustic Data}

The conclusion that even small amounts of array tilt are important in the modal decomposition problem has major consequences for vertical array design. In a different way, it also is significant for the FRAM IV vertical array data to be analyzed here. Since no field measurements of vertical array tilt were taken during the experiment, one must devise a scheme to extract the effective array tilt from the acoustic data if further progress is to be made. The present section presents such a scheme for recovering the effective tilt angle. Such an approach is obviously not ideal; its ability to provide even approximate results is intimately linked to the underlying quality of the data set. In the present instance, the high signal to noise ratio developed by the preprocessing (typically 20 to $30 \mathrm{~dB}$ ) and the exceptional stability of the received signals provide some hope that some reasonable tilt angle estimates can be made.

To estimate the effective array tilt from the acoustic data, the residual error from the multiple beam least squares beamformer is evaluated as a function of the tilt angle assumed in the beamforming process. Equation (5.7) may be rewritten as

$$
Q_{m i n}^{N O R M}(x)=\frac{\underline{\mathbf{P}}^{+}\left[\underline{\mathbf{I}}-\underline{\overline{\mathbf{E}}}(x) \overline{\mathbf{E}}^{+}(x) \underline{\overline{\mathbf{E}}}(\chi) \overline{\mathbf{E}}^{+}(\chi)\right] \underline{\mathbf{P}}}{\underline{\mathbf{P}}^{+} \underline{\mathbf{P}}},
$$

where the dependence on the assumed tilt angle $x$ has now been explicitly indicated. Additionally, the error in equation (6.9) has been normalized by the total square pressure present in the observed field, $\underline{\mathbf{P}}^{+} \underline{\mathbf{P}}$, so that it now represents a fractional goodness of fit measurement. When $Q_{\min }^{N O R M}(x)$ is evaluated across the range of physically reasonable tilt angles, a consistent global minimum can typically be expected. The tilt angle that generates this minimum can then be used for the estimated tilt of the array. The underlying assumption is that the beamformer is 
better able to match the observed sound pressure field when using the correct array tilt angle than when working with any incorrect tilt value.

It is important to review what the procedure just outlined accomplishes from a processing standpoint. The multiple beam least squares modal beamformer computes the set of complex modal amplitudes that produce the best fit to the observed pressure field for one assumed array tilt. The residual error is a measure of how well this "best fit" actually matches the observed field. Note that the residual error is always smaller than the total square error generated by any other set of modal amplitudes at the assumed tilt angle. If the data is then processed using the tilt angle which minimizes the residual error, the resulting set of amplitudes is guaranteed to produce a better match to the observed field than any other possible combination of modal amplitudes and array tilts. Thus, the minimized error philosophy, which forms the basis for the original beamforming algorithm, has been extended further to cover the array tilt angle.

Synthetic data has once more been employed to study the performance of the scheme. One such result is displayed in Figure 6-7. Here the normalized residual error has been plotted as a function of assumed tilt angle and time. The normalized error has been plotted on a $\mathrm{dB}$ scale, so that the $-10 \mathrm{~dB}$ line marks a $90 \%$ fit and the $-20 \mathrm{~dB}$ line indicates a $99 \%$ fit, etc. As a practical matter, the normalized residual error has been computed as an average value over a two minute window of data, rather than instantaneously (as indicated in equation (6.9)). The computation has been made once each minute, giving a $50 \%$ overlap factor. This windowing procedure is identical to that carried out for synthetic data throughout the thesis.

The synthetic data set from which Figure 6-7 has been created is similar in most ways to those presented previously. For the present figure, modes 1, 3, 5, and 7 of the $47 \mathrm{~Hz}$ mode set have been coherently summed to generate the signal field: 


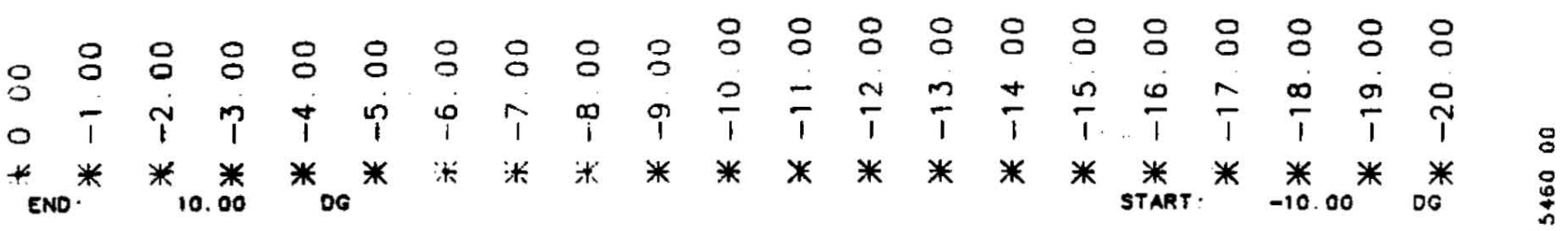

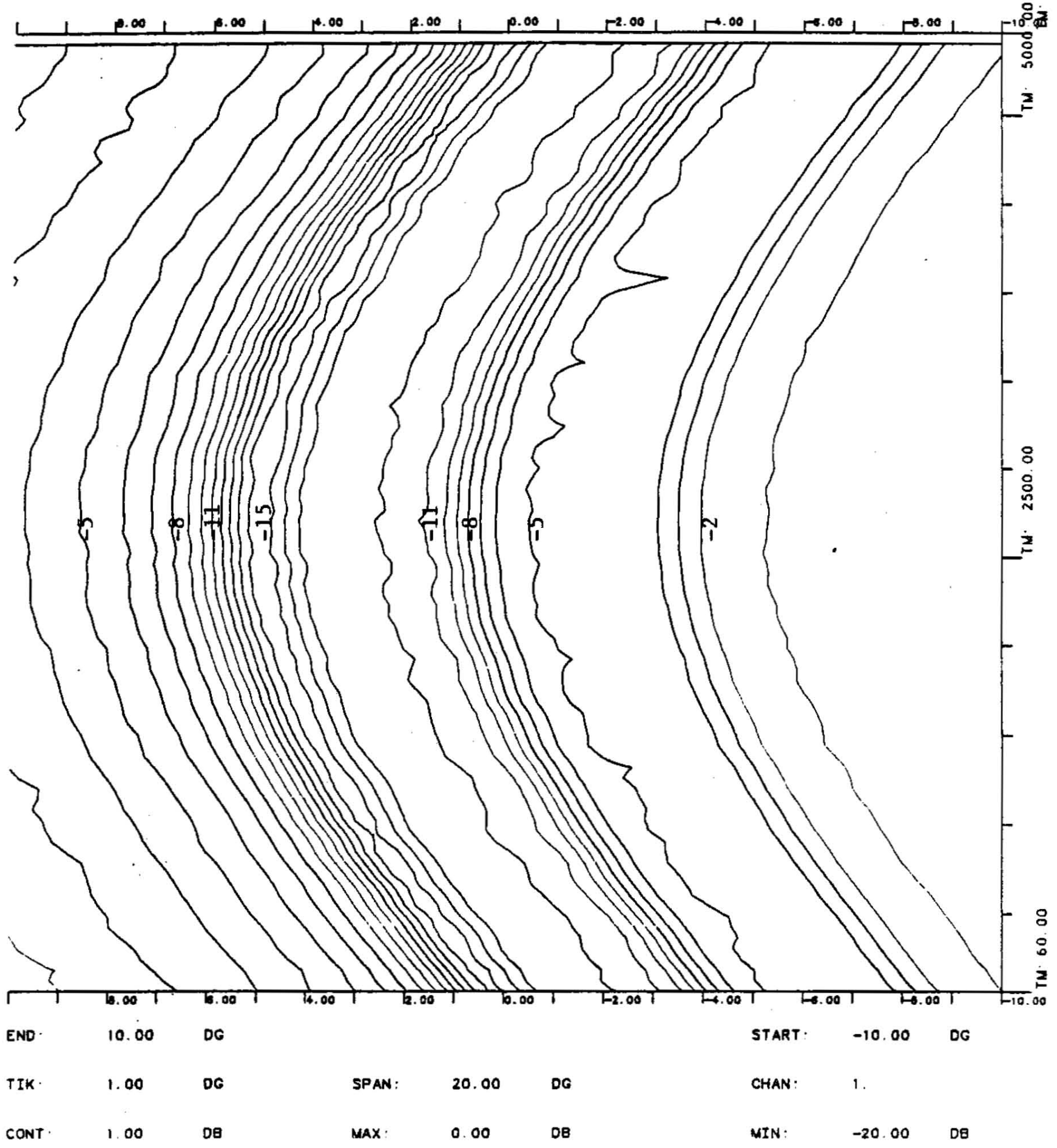

$47 \mathrm{HZ} \mathrm{-} \mathrm{MULTIPLE} \mathrm{BEAM} \mathrm{LEAST} \mathrm{SQUARES} \mathrm{BEAMFORMER} \mathrm{-} \mathrm{COHERENT} \mathrm{SYNTHETIC} \mathrm{DATA}$

Figure 6-7: Normalized Residual Error as a Function of Tilt Angle for Synthetic Data at $47 \mathrm{~Hz}$ (No Excluded Modes Present) 
$-201-$

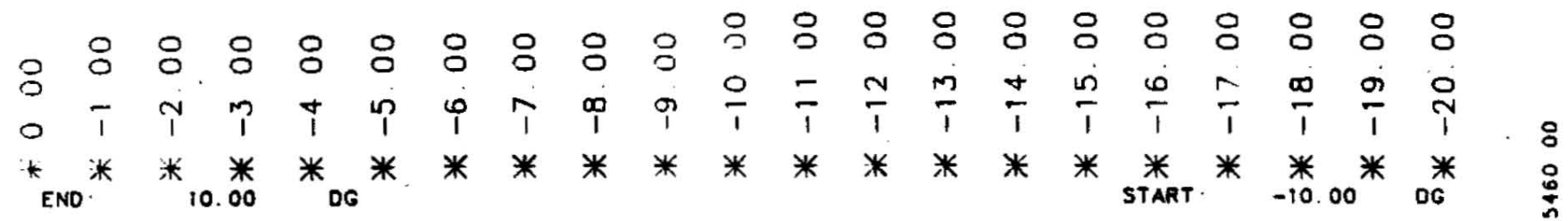

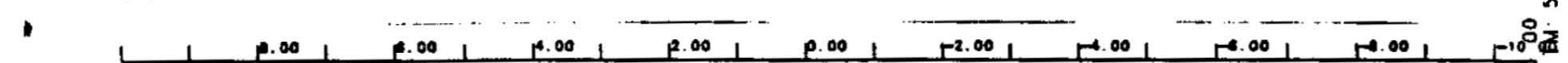

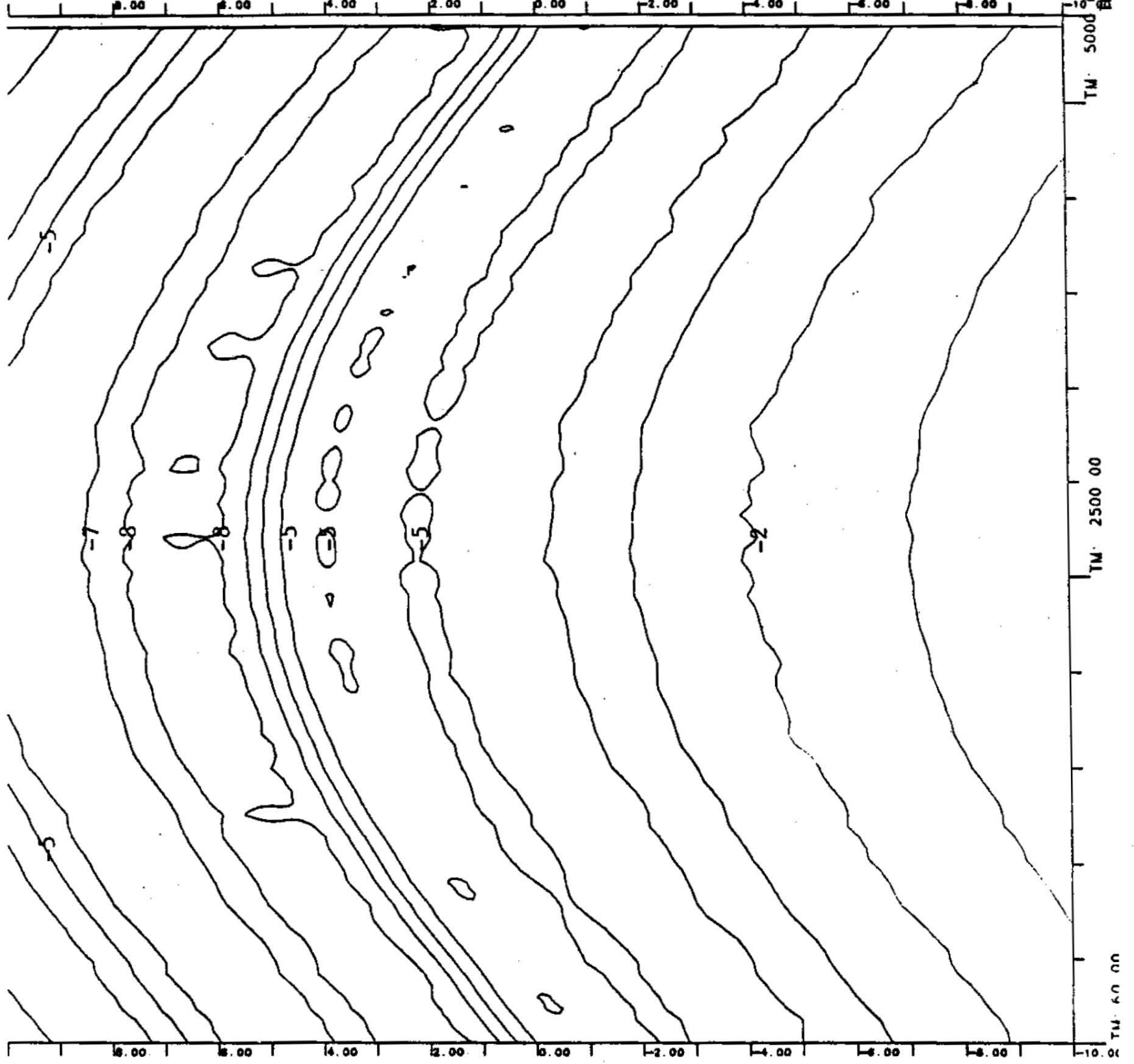

END. $\quad 10.00 \quad 06$

START. $-10.00 \quad$ DG

TIK. $\quad 1.00 \quad$ DG

SPAN: $\quad 20.00 \quad$ DG

CHAN. 1 .

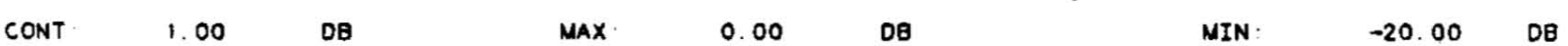

$47 \mathrm{HZ}$ - MULTIPLE BEAM LEAST SQUARES BEAMFORMER - AL IASED SYNTHETIC DATA

Figure 6-8: Normalized Residual Error as a Function of Tilt Angle for Synthetic Data at $\mathbf{4 7} \mathrm{Hz}$ (Excluded Modes Present) 
each mode has been assigned an amplitude of unity. Unlike the synthetic data sets used in Chapter 5, the modal amplitudes shown here are constant and unmodulated over time. Rather, in Figure 6-7 it is the effective array tilt that has been sinusoidally varied over time. This variation occurs over a range from vertical to $5^{\circ}$ and back, and has an oscillation period of 3 hours $(10,800 \mathrm{sec})$. Figure $6-\bar{T}$, therefore, covers the first half cycle of this tilt oscillation.

Figure 6-7 demonstrates the ability of the tilt angle estimation scheme described above to properly track vertical array tilt over time. The minimum errors observed are in the $-15 \mathrm{~dB}$ range, representing a $97^{\circ} \circ$ fit of the synthetically generated data. Note that the minimum error tracks the actual tilt of the array quite well as it swings away from the vertical and then back.

Figure 6-7 is a highly idealized situation, for two reasons: a tilted line array possessing no higher order shape components has been assumed in generating the synthetic field; and only the seven modes included in the beamformer have been injected into the signal. While the effect of the presence of higher order shape components on the array tilt estimation process is difficult to quantify, the fundamental issue underlying it remains the adequacy of the linear tilt model for array shape, which has already been discussed at some length. Figure 6-8 demonstrates the effect of having significant amounts of signal energy carried by modes not included in the beamformer. Here, both the synthetic data and the processing used are identical to those of Figure 6-7, with the lone exception that modes 9, 11, 13, and 15 have additionally been inserted into the signal field at unit amplitude. The presence of this extra energy in modes excluded from the beamformer makes it more difficult for the algorithm to attain a good match with the observed field at any tilt angle, which is reflected by the increased minimum residual error $(80 \%$ in Figure 6-8 versus $97 \%$ in Figure 6-7). It also generat tes a bias 
of about $2^{\circ}$ in the resulting tilt angle estimate. While both the amount and direction of the bias are difficult to predict, its cause is not hard to explain. Even though some energy in the included modes is not accounted for, the biased tilt angle generates less residual error than the true tilt because even more of the energy in the excluded modes is allowed to leak into the result. The amount of the bias is directly reiated to the relative proportions of signal energy present in the modes included and excluded by the beamformer. The actual minimum error level achieved can be used as a measure of this effect. Processing that results in minimum normalized errors of much above $-10 \mathrm{~dB}$ should be regarded with suspicion.

\subsection{Summary}

The least squares modal beamforming algorithm developed in Chapter 5 has been shown to be quite sensitive to sensor position errors, particularly when it is applied to vertical arrays. The effective linear tilt of the array away from true vertical appears to be the component of array shape that contributes most significantly to this sensitivity. Two reasons account account for the effect. The shapes of long, heavy vertical arrays (such as the one deployed during the FRAMIN Arctic Experiment) are composed, in a mean square sense, primarily of low order components, such as constant translations, linear tilts, and parabolic shapes. The zero order constant translation term has no effect on the beamforming problem, and may be completely removed by proper redefinition of the coordinate system. The first order linear tilt term can be expected to account for the majority of the total sensor position error, especially in strong currents where the sensor offsets are greatest. To demonstrate this concept, a model-based notional shape generated for the FRAM IV vertical array has been analyzed using Legendre polynomials. Also. 
the physics of the problem support the argument that the impact of the linear tilt component of the array shape on the modal decomposition process is more significant than that of higher shape components. This is because the array tilt causes each modal beam to effectively "point away" from the vertical directions in which that mode's energy arrives. By contrast, the primary effect of higher order shape components is only to defocus the modal beams.

The above considerations have lead to the selection of a simple tilted line model for the true array shape. Such a model is also attractive because it allows the assumed shape to be completely specified with a single parameter, the effective array tilt angle. This simplicity holds even when the three-dimensional nature of the problem is considered.

The sensitivity of vertical array modal beamforming to array tilt has been studied both theoretically and through the use of synthetic data. A simple method has been developed to estimate the accuracy to which one must know the tilt angle if valid modal decompositions are to be assured. This method implicitly assumes that a rough equivalence exists between each mode and a corresponding pair of plane waves. The applicability of the resulting accuracy estimates is dependent upon the degree to which this equivalence holds in practice. The equivalence may be tested qualitatively by examining the Fourier transform of each mode shape: if a single strong peak exists over some narrow range of vertical wavenumbers, then a reasonable equivalence exists. This appears to be the case for the central Arctic mode set of Chapter 4. Processing results from synthetic data have been utilized to actually demonstrate the sensitivity of the processing to array tilt.

Application of this tilt accuracy estimation method to the FR.AII $\Pi^{\circ}$ verticial array shows that the array tilt must be known to about $\pm 0.25^{\circ}$ to gilarantee meaningful modal separation. These results are in good agreement with tilt angle 
sensitivities demonstrated by synthetic data. They also correspond well to the tilt sensitivities observed in real data taken from the vertical array, which are presented in Section 7.3.

Since no field measurements of vertical array tilt were made during the FRAM IV Experiment, a scheme has been developed to recover tilt values from the acoustic data itself. This scheme involves evaluation of the residual beamforming error as a function of the tilt angle assumed in the beamforming computations. The residual error is a measure of the difference between the the sound pressure field observed at the sensors and the field synthetically generated from the complex amplitude outputs of the modal beamformer. As such, it indicates how well the outputs of the beamformer match the observed pressure field. Thus, the tilt that generates the smallest residual error is the one which best fits the observations, and may be assumed to be the actual vertical array tilt angle.

Through the use of synthetic data, the tilt angle estimation scheme has been shown to work well when applied to high SNR signals. In making this demonstration, an ideal linear shape has been assumed for the array. The low SNR situation has not been studied, but it is expected that performance would be severely degraded under these conditions. The relative amount of signal energy: carried by modes not included in the multiple beam least squares algorithm has been shown to affect the accuracy of the estimate. When the contribution of these modes is negligible, the method can be expected to provide accurate estimates; as the contribution becomes more important, a bias is injected into the estimate. leading to progressively poorer performance. When significant signal enterg is resident in modes, not included in the beamformer, higher values of the normalized residual error are also generated, even at the minimum. This is because the beamformer has increasing difficulty in matching the total field with just the modes 
-206-

available to it. Thus, the value of the normalized residual error at the minimum can be used as a guide to the accuracy of the tilt angle estimate. Fits of much less than $90 \%$ (greater than $-10 \mathrm{~dB}$ normalized error) should be considered to be suspect. 


\section{Chapter 7}

\section{Data Presentation and Analysis}

The purpose of this chapter is to present the results obtained from applying the processing techniques outlined in Chapters 5 and 6 to the data set described in Chapter 3, based on the modal structure of Chapter 4. In so doing, a number of different hypotheses can be tested. First, the validity of the assumed modal structure can be established. Second, the results of processing the vertical array data can be compared to those generated from the more traditional horizontal array processing, providing a qualitative measure of the vertical array processing performance. These efforts generate confidence in the computed mode properties and the processing performance, after which a full analysis of the implications of both the horizontal and vertical array results can be undertaken.

The processing output includes three different types of useful data. The absolute mode amplitudes are directly available. Their relative sizes provide immediate insight into the nature of low frequency Arctic propagation, and they call be used to compute observed values of the modal attenuation coefficients. Comparison of these coefficients with predicted values sheds light on the channel parameters that most influence Arctic sound transmission. The time series of the amplitudes also provide some general indications of the temporal stability of the central Arctic channel.

Measurements of mode coherence provide data of a different type, and indicate whether the signal's component modes are phase-random or phase-locked with respect to each other. This data can be used to test the uncorrelated multipath assumption that is widely used in theoretical developments. 
Finally, if the different modes do maintain a deterministic phase relationship. then it is meaningful to measure the relative phases between various pairs of modes. These relative phase values provide a measure of the range dependence of the channel, since they represent an integration of horizontal phase speed fluctuations that the modes have encountered during propagation (see equation (2.30)). Thus. if the measured values disagree with values predicted from the phase speed data of Figure 4-10, then the channel must be assumed to show non-negligible variations in range; if the agreement is good, the simpler range independent channel model will suffice.

This chapter is organized into five major sections, some containing multiple subsections. Following some preliminary comments, the horizontal array data processing results are presented and summarized. The section is divided into four subsections; one deals with azimuthal beamforming and another with verticil steering. The third summarizes the results across the full data set. In the last subsection, the predicted first mode horizontal phase speeds are compared with those observed from the horizontal array data, so that the accuracy of the modal characteristics computed in Chapter 4 can be assessed.

A similar section summarizes the results for the vertical array. Two subsections discuss the array tilt problem and the tilt angle estimates made with the procedure discussed in Section 6.6. The third presents some typical modal amplitude levels versus time, while the fourth discusses observations of possible array shape changes over time that can be observed in the data.

Following this are three sections dealing with data analysis. The first of these sections investigates the modal amplitude data. One subsection compares the horizontal and vertical array results. The second analyzes the observed modial attenuation coefficients versus theoretical predictions, and the last subsection 
contrasts both sets of results with some shot data available for the same channel. The second section presents and discusses the mode coherence estimates made. The final section presents the relative phases observed between various modes. A summary of major points completes the chapter.

\subsection{General Comments}

Before commencing the actual presentation of processing results, some general comments concerning the formats used and the processing parameters selected are in order. First, all amplitude levels shown in this chapter are displayed on a decibel scale referenced to units of absolute pressure (1 $\mu \mathrm{Pa}$ rms); conversion from the voltage levels measured has been done using the hydrophone sensitivities quoted in Figure 3-6 and Table 3-III. A logarithmic scale is also used for displaying residual error data; the values shown are 10 times the logarithm of the normalized residual error, as defined in Section 6.6. An error level of $-10 \mathrm{~dB}$ represents a $10^{\%}$ error. or, equivalently, a $90 \%$ fit to the observed data, while a level of $-20 \mathrm{~dB}$ represents a $99 \%$ fit. Phase data are presented on a linear scale from $-180^{\circ}$ to $180^{\circ}$; phase wrap is often observed, as only the principal value of the phase is displayed. Coherence measurements are presented in normalized form, so that the values range from 0.0 to 1.0 .

On all plots, amplitudes or residual error values are marked with DB. Mode numbers (such as mode 1 , mode 2 , etc.) are denoted by MD, while horizontal phase speeds (in $\mathrm{m} / \mathrm{sec}$ ) are indicated by PV. DG is used for azimuth angles on plots of horizontal array data and for array tilt angles on plots of vertical array data. All azimuth angles are measured with respect to the convention discussed in Section 3.3; the tilt angles displayed are effective angles measured in the vertical plane of propagation (see Section 6.1). Where axes are indexed by node number, it is 
important to remember that data only exists at the integer values; any markings between these discrete points, such as connecting lines on one-dimensional plots or contours on two-dimensional plots, have no physical significance and are included only to help in judging relative trends by eye. Where contoured data indexed by mode have been presented, overlying black lines have been added to the plot to emphasize its discrete nature. All levels plotted versus mode number are modal amplitudes. For the horizontal array, levels plotted versus horizontal phase speed are plane wave amplitudes, and differ from modal amplitude measurements by a factor equal to the size of the mode shape at the $91 \mathrm{~m}$ depth of the array. The necessary conversion factors can be found in Figure 4-8.

Processing windows of $28.8 \mathrm{sec}$ (18 points, providing a time-bandwidth product of 0.7 ) have been used for estimating the sensor cross-coherence matrices for horizontal array data. One set of beams is computed every 15 seconds, providing roughly a $50 \%$ overlap factor. With some noted exceptions, vertical array data have been processed in 120 sec windows ( 75 points, giving a time-bandwidth product of 3.0 ), with a computation interval of $60 \mathrm{sec}$, again giving a $50 \%$ overlap. The very stable and coherent nature of the Arctic sound channel makes the difference negligible. No windowing functions (such as Bartlett or Hanning windows) have been employed in the processing.

The 17.75 and $47 \mathrm{~Hz}$ frequency data from both the horizontal and vertical arrays have been chosen for display throughout the chapter, since they are reasonably representative of the results at other frequencies. This is done to allow direct comparison of the various processing results while limiting the chapter to an acceptable length. Results from other frequencies are presented when they demonstrate unique features of interest. The most important results are summarized by tables or plots that span the full range of available frequencies. 


\subsection{Presentation of the Horizontal Array Processing Results}

The data taken from the horizontal array have been processed using both the single beam MLM beamformer and the conventional (single beam least squares) beamformer. In general, the MLM algorithm provides much higher resolution than the conventional approach, but does not produce reliable quantitutive measurements, because of the presence of coherent interference. Quantitative amplitude estimates of observed MLM peaks have been made by reprocessing the data using the conventional beamformer. This technique works best when a strong single peak is present, and is considerably less reliable when closely spaced multiple peaks of roughly equal height are present.

\subsubsection{Aximuthal Beamforming}

Figure 7-1 displays the output of the single beam MLM processor versus time for the $27 \mathrm{~Hz}$ tone from the horizontal array data set. For an assumed sound speed of $1450 \mathrm{~m} / \mathrm{sec}$, the beamformer successively scans through 360 beams spaced 1 apart in horizontal azimuth angle. Note that although 360 beam outputs are being evaluated at each time step, every beam is still being computed individually; a multiple beam algorithm would compute a number of beams all with a single set of matrix operations.

For the azimuthal beamforming done here, the MLM processor is preferable to the conventional beamformer for two reasons: it provides much higher resolution: and it does a significantly better job of suppressing side lobes. Quantitative level measurements are not an issue here. In Figure $7-1$, the tonal signal is readily apparent at an azimuth angle of $86.4^{\circ}$. A low level sidelobe that is down about by about $8 \mathrm{~dB}$ is visible at $270^{\circ}$. This is a back lobe generated by the north-south leg of the array, which is essentially broadside to the incoming signal. 


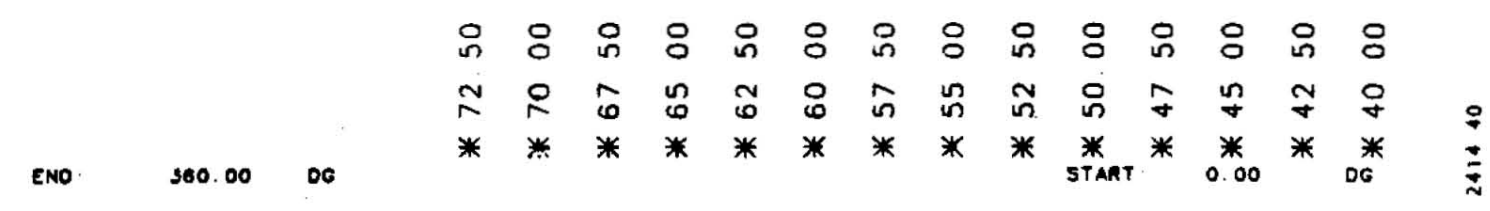

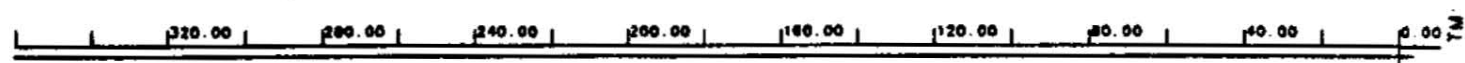

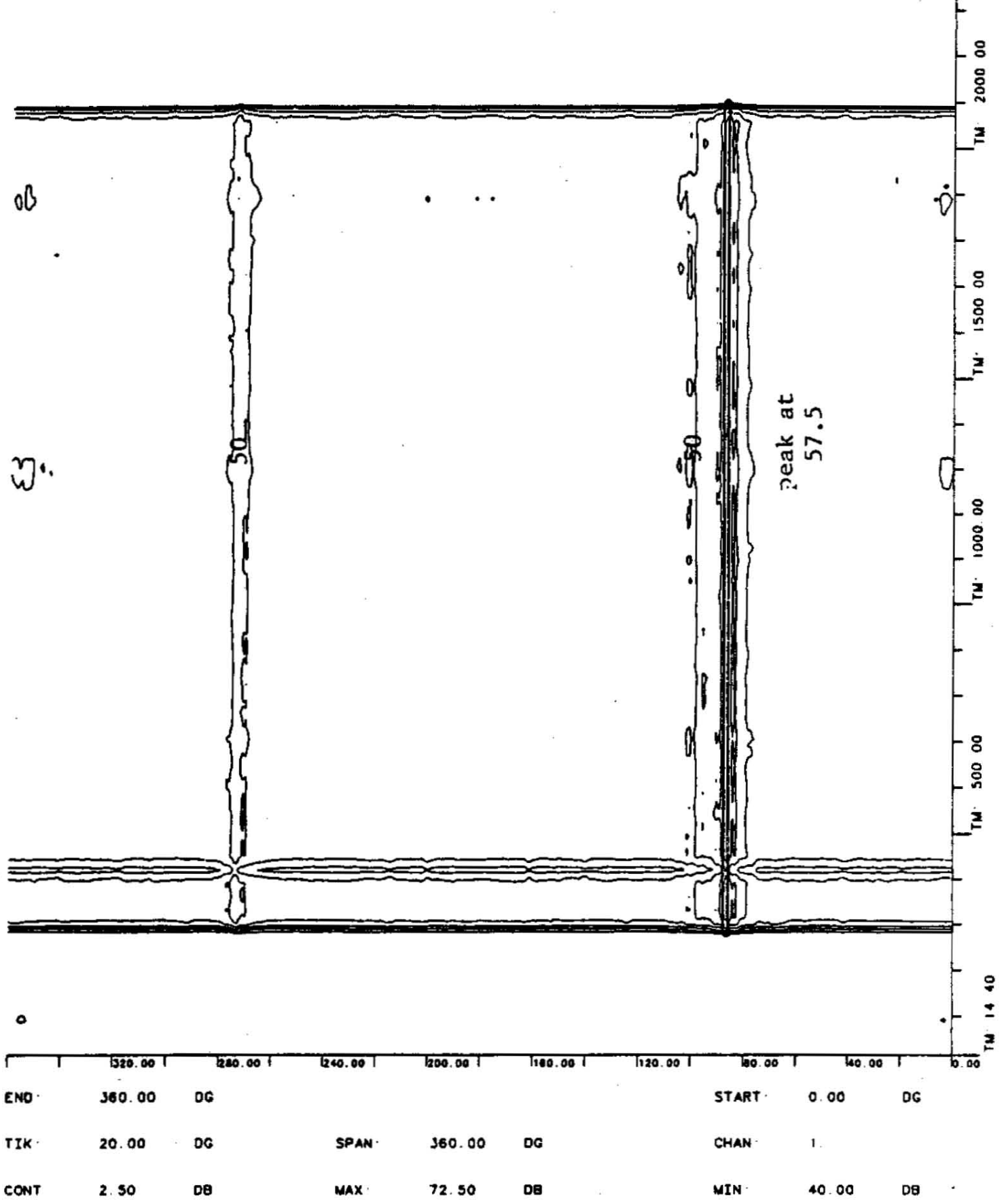

SINGLE BEAM MLM BEAMFORMER STEEREO IN HORIZONTAL FOR 27.00 HZ TONE

Figure 7-1: Output of Single Beam MLM Beamformer Scanned in Azimuth for $27.00 \mathrm{~Hz}$ Tone (Horizontal Array) 
By processing the data with beams spaced $0.1^{\circ}$ apart, one can resolve the source azimuth angle to about $\pm 0.1^{\circ}$, an accuracy not achievable using a conventional beamformer. For comparison, the navigation data provide an azimuth angle estimate of $86.1^{\circ}$ (see Table 3-I). The difference is well within the limits of accuracy of the array rotation measurements, which were made daily from the sun. Similar agreement is obtained for other horizontal array tones.

With one exception, the tone is extremely stable over its full thirty minute duration. That exception is the fade that can be seen at the $420 \mathrm{sec}$ mark. The fade appears to be caused by an instananeous shift in the phase of the received signal, which in turn causes the signal frequency to walk out of the filter passband for a short time. In many respects, it is similar to the fade observed by Mikhalevsky [57]. Neither the azimuthal beamforming outputs nor the vertical steering results (which are shown in Figure 7-2) display any noticeable difference between the spatial structure before and after the event. This observation argues against the assumption that the fade represents a shift in transmission path structure; some type of interruption of the source seems more likely. Background noise variations can be seen to occur in the absence of the signal and during the fade. These are processing artifacts caused by variations in the amount of white noise used to stabilize the cross-coherence matrix estimate.

\subsubsection{Vertical Beamforming}

Figure $7-2$ shows the output of the single beam MLM processor versus time for the $27 \mathrm{~Hz}$ tone. Unlike Figure 7-1, however, the beamformer now scans in the vertical at the previously determined signal azimuth. This is accomplished by stepping the beam's assumed horizontal phase speed from $1400 \mathrm{~m} / \mathrm{sec}$ to $1600 \mathrm{~m} / \mathrm{sec}$ in $1 \mathrm{~m} / \mathrm{sec}$ increments, with the higher phase speeds corresponding to larger 

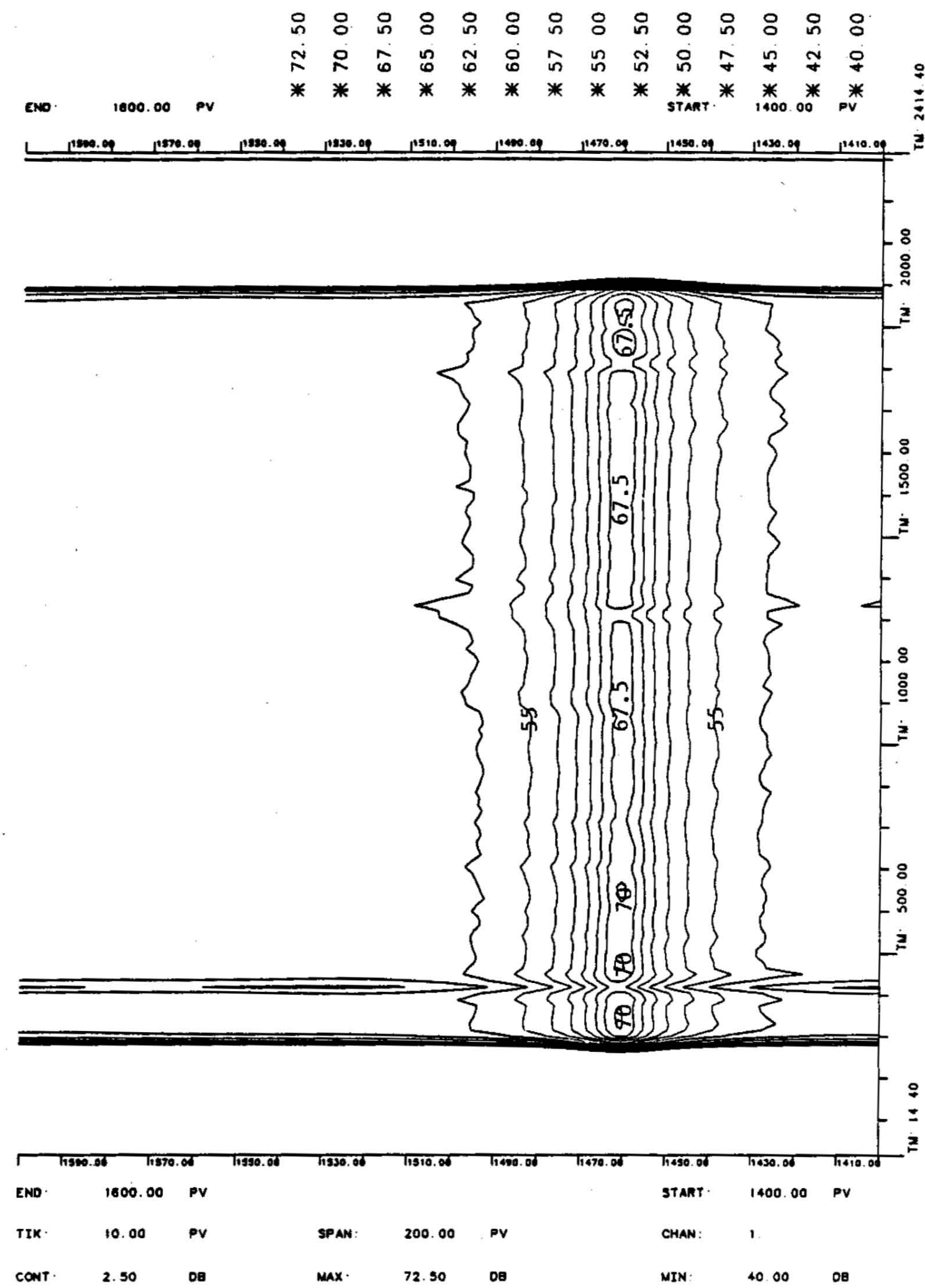

SINGLE BEAM MLM BEAMFORMER STEERED IN VERTICAL FOR 27.00 HZ TONE

Figure 7-2: Output of Single Beam MLM Beamformer Scanned in Phase Speed for $27.00 \mathrm{~Hz}$ Tone (Horizontal Array) 


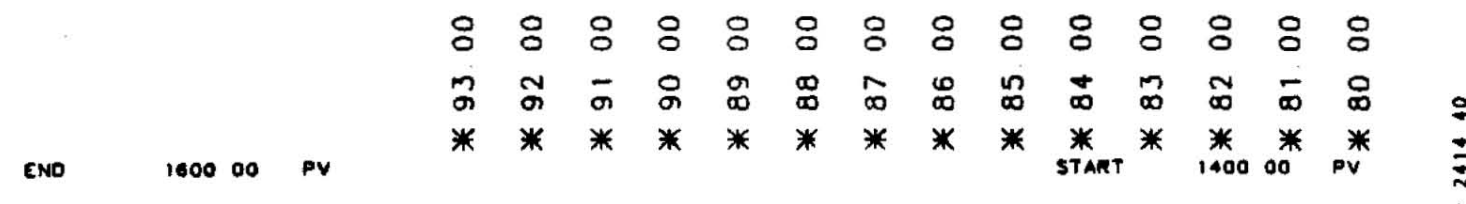

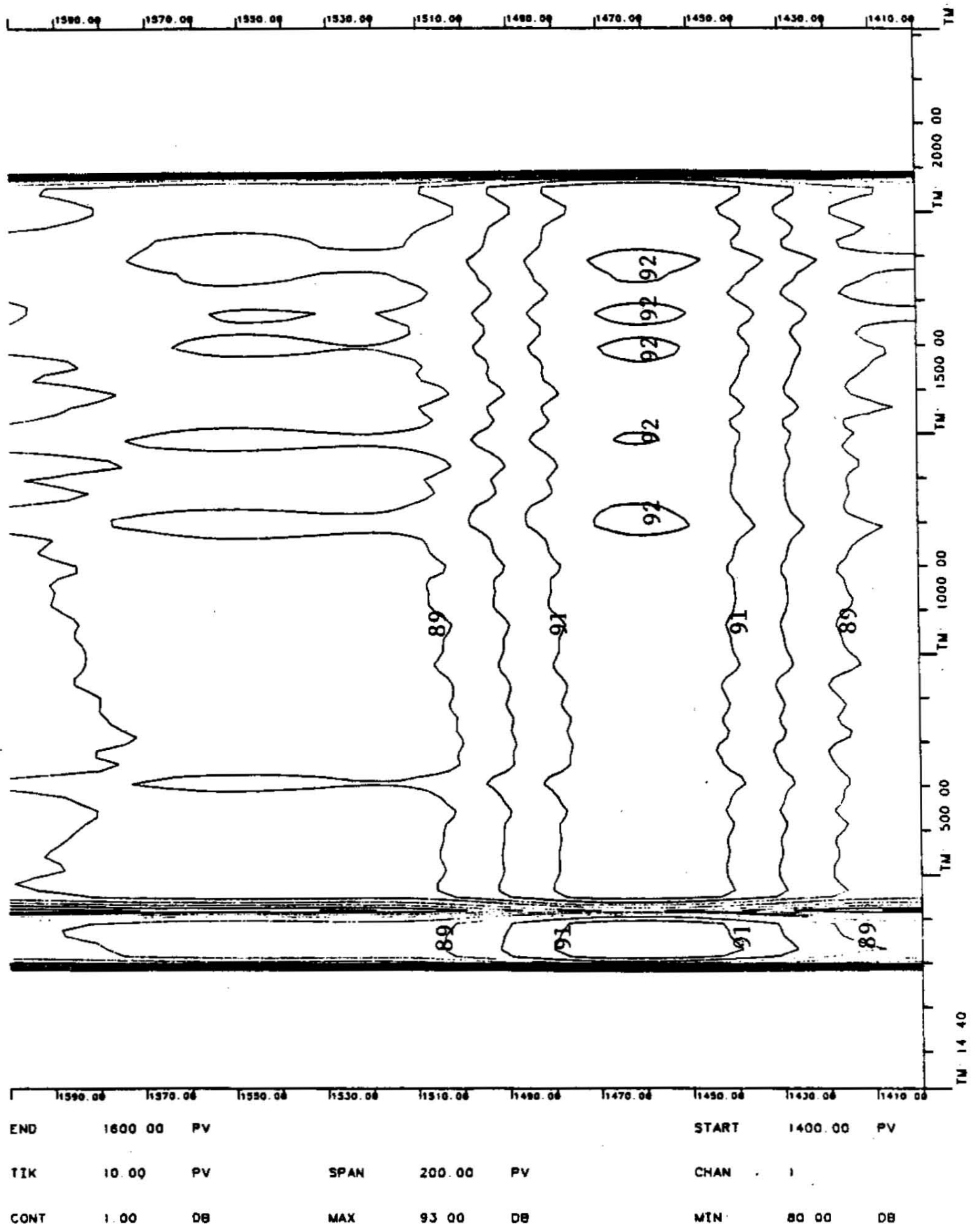

CONVENTIONAL BEAMFORMER STEERED IN VERTICAL FOR 27 OO HZ TONE

Figure 7-3: Output of Single Beam Least Squares Beamformer Scanned in Phase Speed for $27.00 \mathrm{~Hz}$ Tone (Horizontal Array) 
equivalent grazing angles in the beams formed. The signal appears to be composed almost entirely of first mode propagation arriving at about $1460 \mathrm{~m} / \mathrm{sec}$. Such a result is expected, given the analysis of Section 4.5. If the attenuation of the various modes is assumed to be equal, the received level of the first mode at $n 1 \mathrm{~m}$ should dominate its neighbors by $27 \mathrm{~dB}$ (twice the value plotted in Figure 4-9).

Figure $7-3$ shows the same $27 \mathrm{~Hz}$ data processed in the vertical at the signal azimuth, but now using the conventional beamformer. By comparing this figure with Figure 7-2, one can obtain a feel for the resolution gains possible with the MLM algorithm when it is applied in a carefully controlled fashion. Figure $7-3$ is useful for measuring the the first mode's average received signal level (about $91 \mathrm{~dB}$ ), and its fluctuation range (which appears to be no more than $1 \mathrm{~dB}$ ). The complete saturation of the beamformer output by the first mode arrival makes estimates of higher mode levels impossible.

In Figures 7-4 and 7-5, the output of the single beam MLM processor is again presented versus time. Here the $17.75 \mathrm{~Hz}$ tone recorded on the horizontal array has been processed, and the beamformer has been steered in the vertical at the appropriate azimuth angle. The plots indicate some of the practical difficulties involved in properly assessing channel stability. Figure 7-4 was generated first, by including all 24 hydrophones available from the horizontal array in the beamforming. In an effort to assess the nature of the fluctuation seen about half way into the signal, the preprocessed time series data were carefully reviewed on a sensor by sensor basis. Three different sensors (not adjacent to one another) wert found to exhibit abnormally high signal levels for short periods near the time of the disturbance, and have been eliminated as inputs to the beamformer in the remults displayed in Figure 7-5. The previous fluctuation has now been completely eliminated. The cause of the fluctuations on the three sensors is not understood. 


$$
\begin{aligned}
& \text { กิ } 8 \text { in } 8 \text { in } 8 \text { i } 8 \text { in } 8 \text { i } 8 \text { in } 8
\end{aligned}
$$

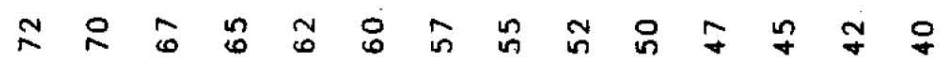

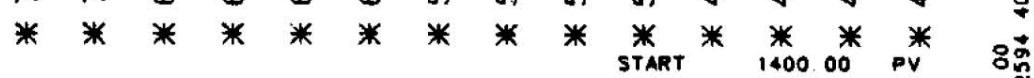

$$
\begin{aligned}
& \text { ENO } 1600.00 \mathrm{PV} \text { START } 1400.00 \text { PV } 80 .
\end{aligned}
$$

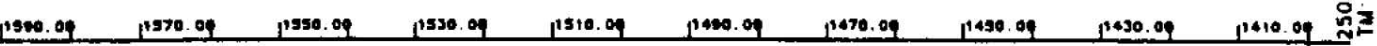

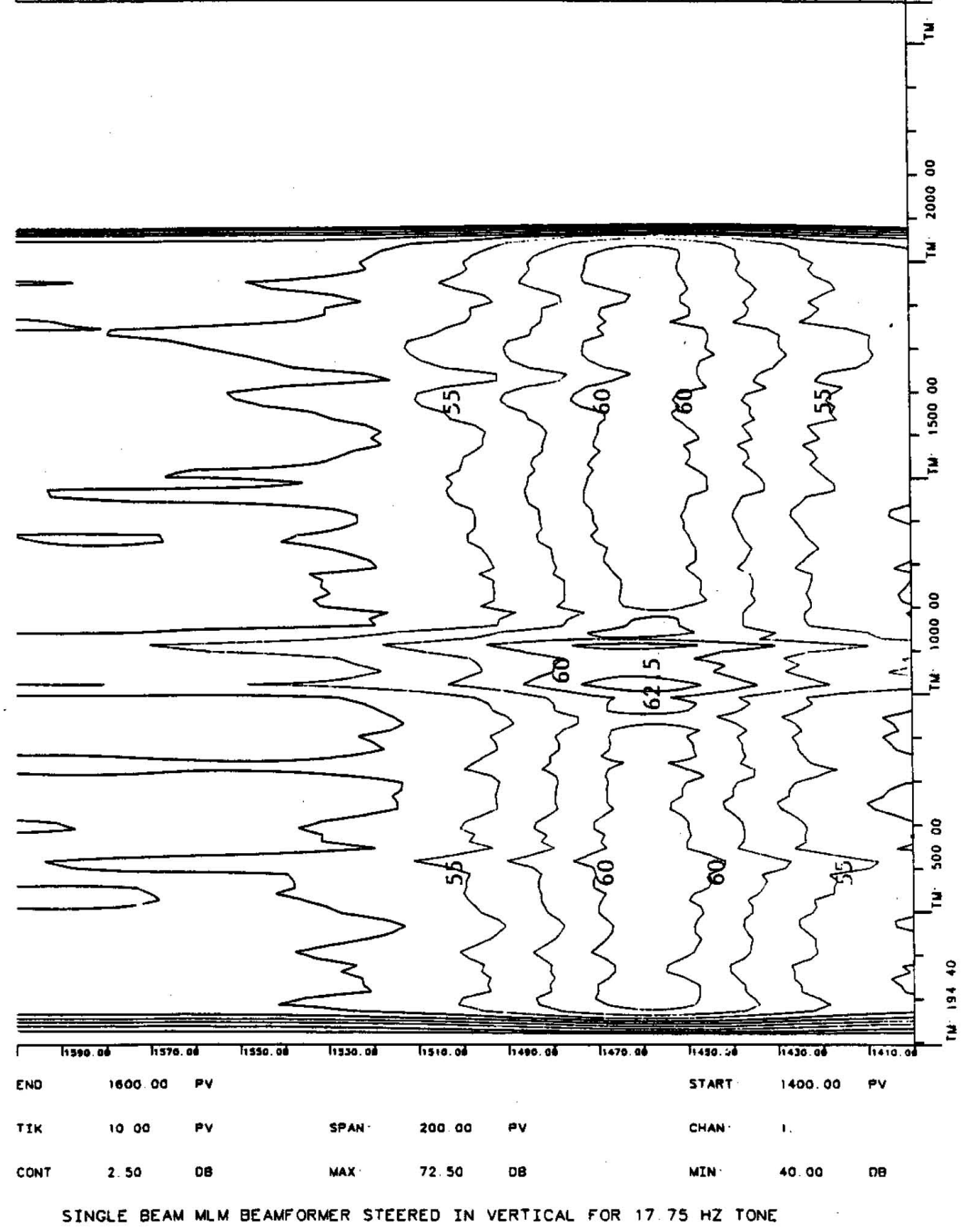

Figure 7-4: Output of Single Beam MLM Beamformer Scanned in Phase Speed for 17.75 Hz Tone (Horizontal Array) 


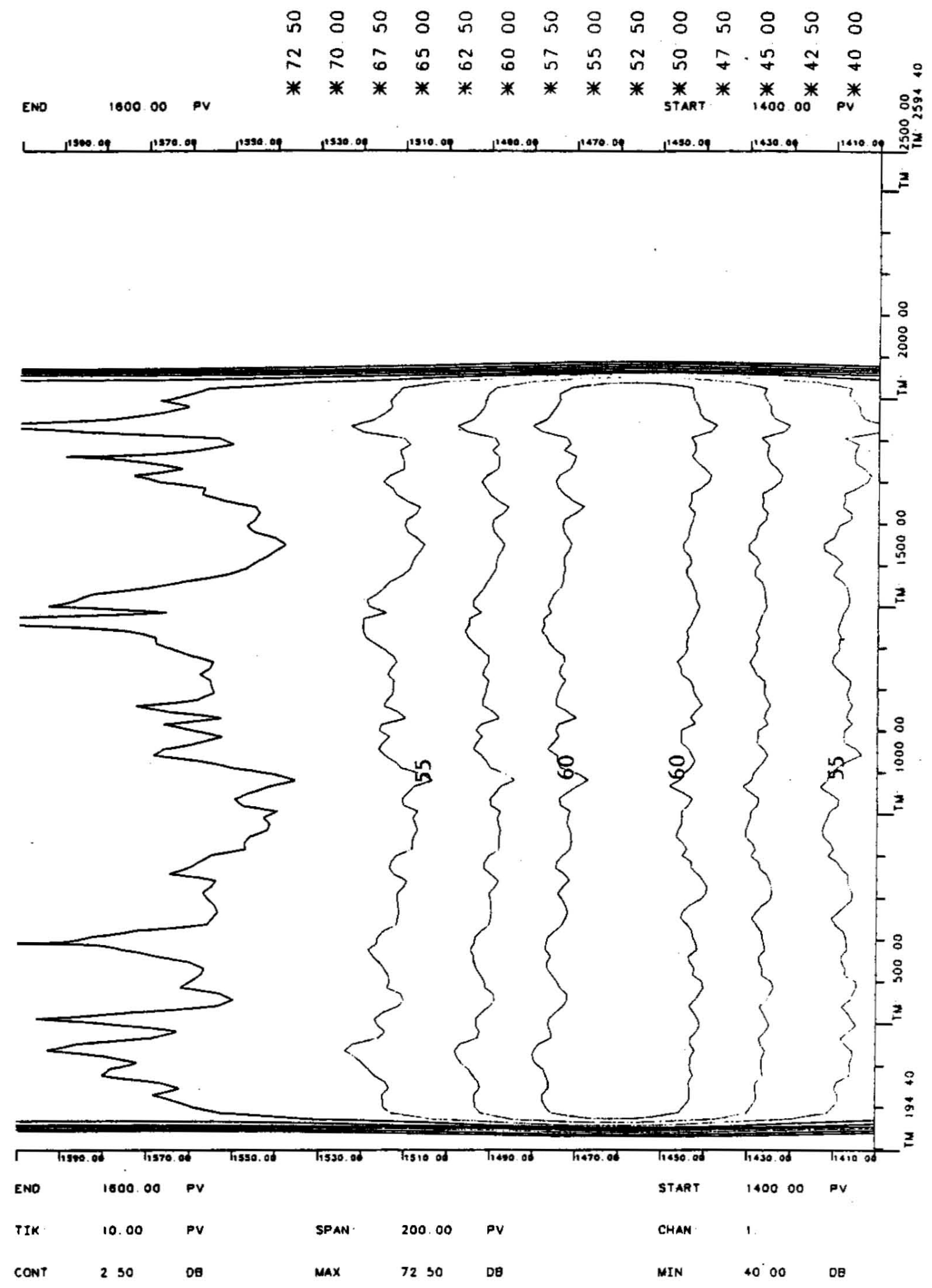

SINGLE GEAM MLM BEAMFORMER STEERED IN VERTICAL FOR 1775 HZ TONE

Figure 7-5: Output of Single Beam MLM Beamformer Scanned in Phase Speed for $17.75 \mathrm{~Hz}$ Tone

(Horizontal Array with 3 Sensors Excluded) 
However, the signal level variations point more to a mechanical or electrical phenomenon than an acoustic cause. Such variations have also been observed in other data taken from the horizontal array.

The results of Figure 7-5 are similar to those obtained at $27 \mathrm{~Hz}$, and once again show dominant first mode propagation. This is in qualitative agreement with the preliminary analysis done in Section 3.12 for the $17.75 \mathrm{~Hz}$ tone recorded on the vertical array. The widening of the first mode peak in Figure $7-5$ compared with Figure 7-2 is attributed to the loss of array directionality at the lower frequency.

The final signal displayed from the horizontal array data set is the $47 \mathrm{~Hz}$ tone shown in Figure 7-6. This figure has again been generated from the output of the MLM beamformer when steered in phase speed at the signal azimuth. Note the marked lack of a strong first mode arrival, which should appear at a phase speed of about $1449 \mathrm{~m} / \mathrm{sec}$. Instead, the most intense arrival occurs near $1480 \mathrm{~m} / \mathrm{sec}$, and can be associated with the deepest diving RSR rays of the TRISTEN/FRAMI channel (see Figure 4-12). Even this arrival is only marginally stronger than those seen at other phase speeds, though. The received levels appear to indicate a rough equipartition of energy in the various modes; however, the array lacks sufficient resolution in the vertical to make a definitive statement about the distribution of energy in the higher modes. In any case, the strong first mode arrival seel at lower frequencies has now disappeared.

This result agrees closely with the initial analysis of the $47 \mathrm{~Hz}$ tone from the vertical array done in Section 3.12, and is quite remarkable when one considers that $47 \mathrm{~Hz}$ lies almost at the peak of the relative advantage curve shown in Figure 1-9. The theory predicts that the first mode should dominate its neighbors by almont 60 $\mathrm{dB}$ if the attenuation is constant across all modes, which is in direct opposition to the analysis completed both here and in Section 3.12, where it is concluded that the 


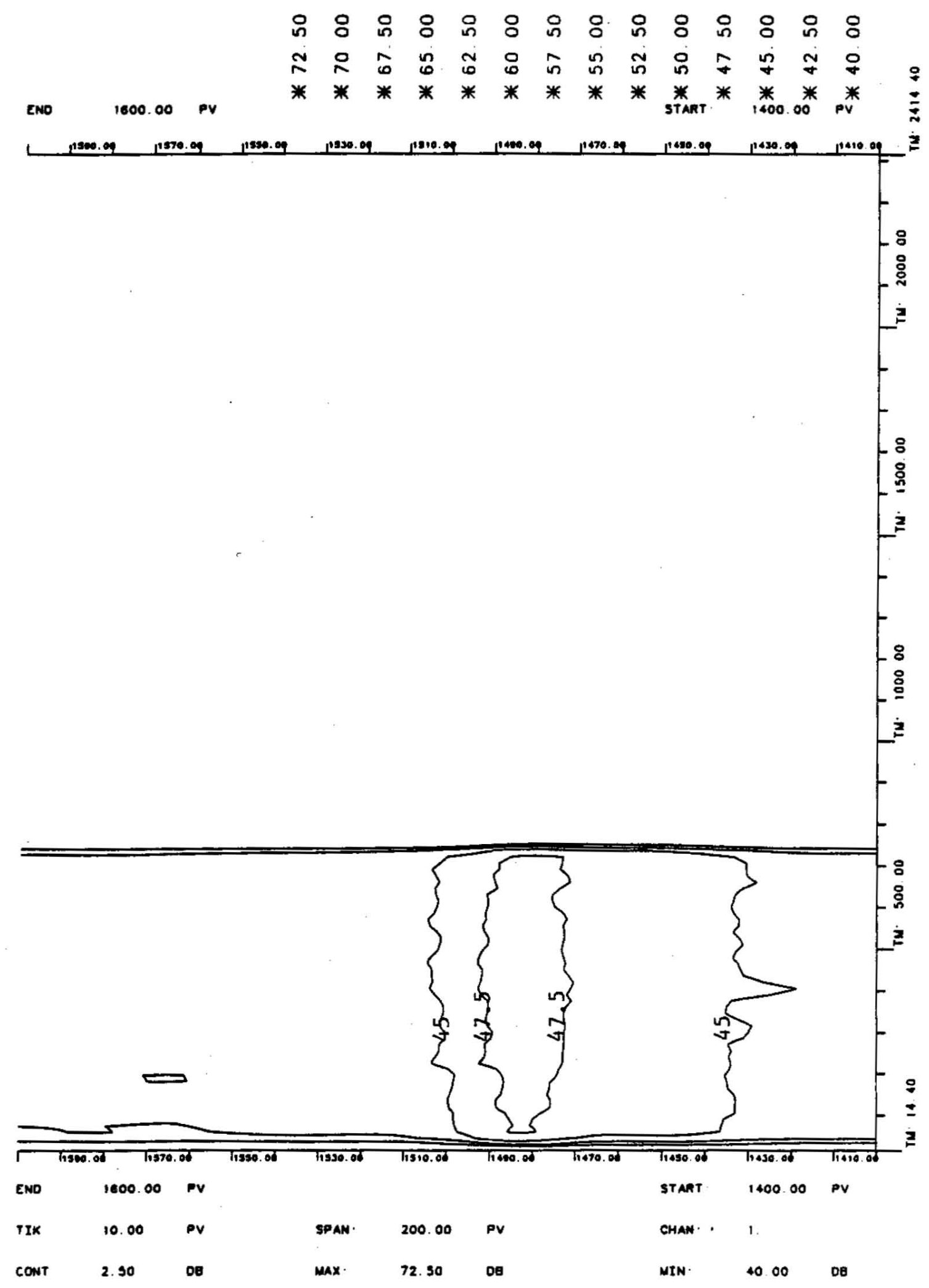

SINGLE BEAM MLM EEAMFORMER STEERED IN VERTICAL FOR $47.00 \mathrm{HZ}$ TONE

Figure 7-6: Output of Single Beam MLM Beamformer Scanned in Phase Speed for $47.00 \mathrm{~Hz}$ Tone (Horizontal Array) 
variations of the received signal with depth can only be explained by the presencen of a number of coherently interfering modes. The $53.25 \mathrm{~Hz}$ tone from the horizontal array data set indicates similar low levels for the first mode.

\subsubsection{Summary of the Horizontal Array Processing Results}

Several important general observations can be made from the procesived horizontal array data. An extremely stable channel has been observed at all frequencies in the data set below $70 \mathrm{~Hz}$. Fluctuations in beamformer outputs orer periods as long as 30 minutes rarely exceed $1 \mathrm{~dB}$. The data appear to be separable into two or, possibly, three regimes. The data below about $30 \mathrm{~Hz}$ show the expected dominant first mode propagation. Despite the tremendous relative advantage possessed by first mode's coupling to both the source and the horizontal array, the data above $40 \mathrm{~Hz}$ show only very weak first mode propagation. In this frequency regime, the deep RSR rays of the channel appear to contribute marginally more to the total propagation than does any other path. While a strong first mode arrival is also seen in the region between 30 and $40 \mathrm{~Hz}$, it is perhaps not as dominant as might be expected from the results of Section 4.5. This frequency region appears to be a transition zone between the other two propagation regimes. The primary source of this conclusion is the data from $35.25 \mathrm{~Hz}$ signal, which has not been displayed here.

The vertical resolution of the horizontal array is inadequate to allow separation of the individual modes, even when the higher resolution MLM algorithm is employed. At frequencies below $40 \mathrm{~Hz}$, good quantitative estimates of the received level of the first mode can be made because of its relative strength compared to other modal arrivals; this same dominance prohibits estimates for higher modes at these frequencies. At frequencies above $40 \mathrm{~Hz}$, all mode level 
measurements are of questionable accuracy because of the lack of a dominant propagation path.

The result at $70 \mathrm{~Hz}$ is a special case. While an investigation of the complex time series output from the preprocessor shows a signal of reasonable strength to be present, neither the MLM nor the least squares beamformers produce acceptilble outputs. The inability to beamform this data effectively is attributed to the sermor displacement problem discussed in Section 3.7.

\subsubsection{Verification of the Modal Structure}

The high resolution of the single beam MLM beamformer allows experimental observations of the first mode horizontal phase speed to be made for signal frequencies below $40 \mathrm{~Hz}$. These measurements can then be used to verify the modal structure derived in Chapter 4. Figure 7-7 summarizes this data. Here, both the computed and the observed first mode phase speeds are plotted as a function of frequency. The error bars that are plotted with the experimentally determined values indicate the approximate width of the first mode peak seen in the MLM processing output. With the exception of the $27 \mathrm{~Hz}$ result, the agreement between prediction and observation is more than adequate. The $27 \mathrm{~Hz}$ data point is obviously out of line with both the computed results and the other experimental data, but the cause of this disagreement is not understood. Since the phase speeds are much more sensitive to environmental variations than the mode shapes, one can conclude from Figure 7-7 that the results presented in Chapter 4 adecinately describe the actual modal structure at the receiving arrays. 


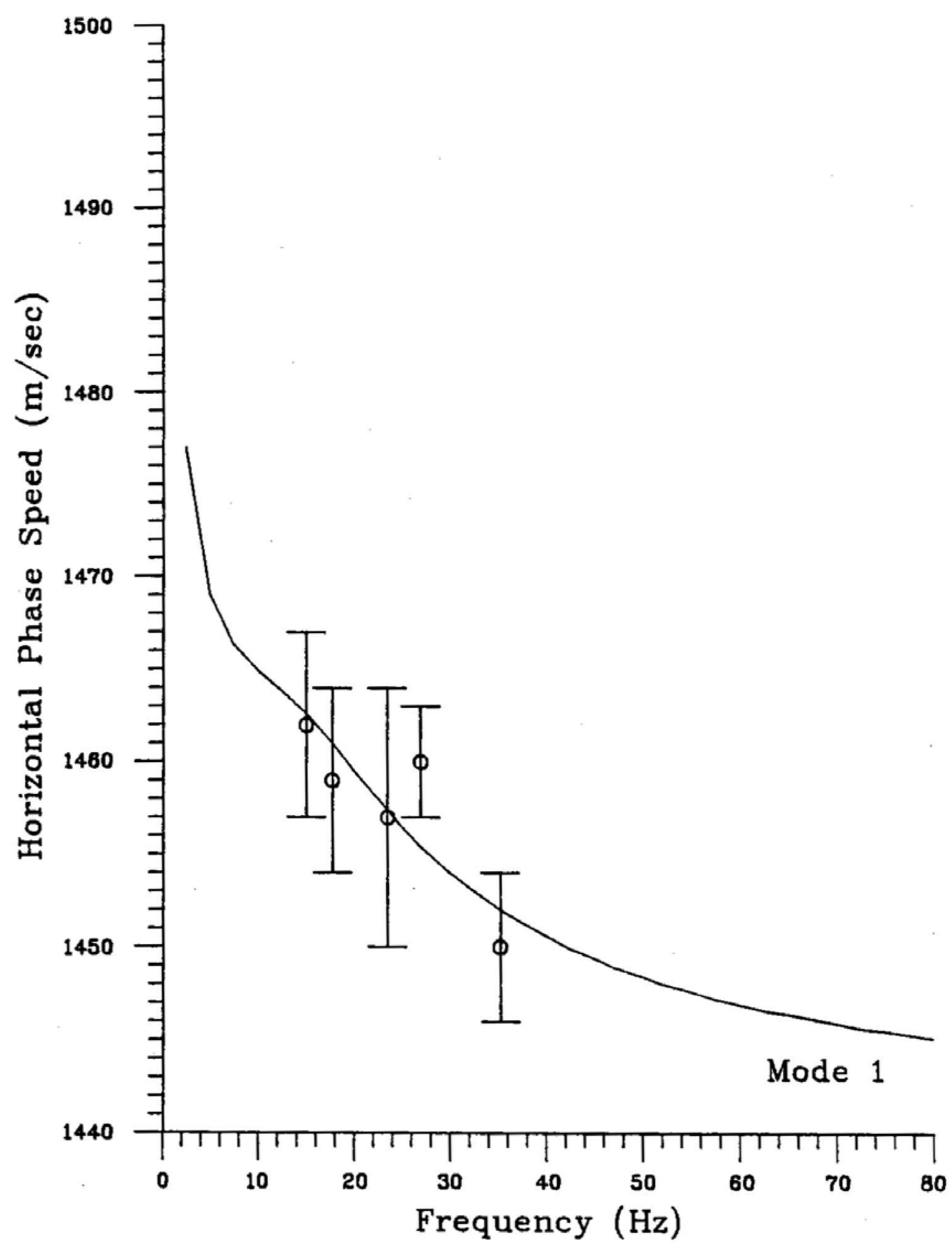

Figure 7-7: First Mode Phase Speed versus Frequency; C'omparison of Horizontal Array Observations with Theory 


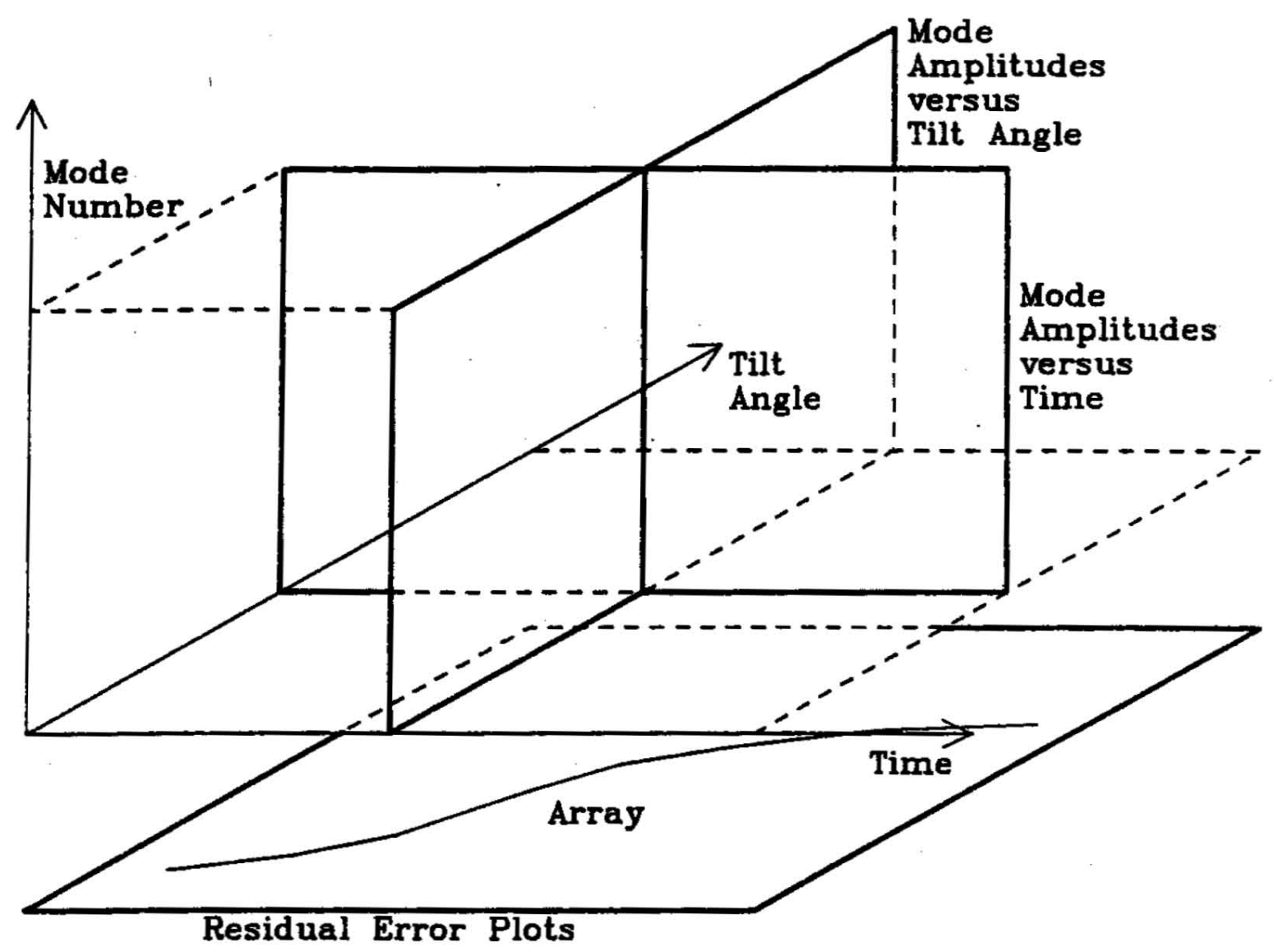

Figure 7-8: Conceptual Relationship of the Different Types of Vertical Array Processing Outputs

\subsection{Presentation of the Vertical Array Processing Results}

In keeping with the discussion of Chapter 5 , the data taken from the vertical array have been processed using a multiple beam least squares beamforming algorithm. Because this beamformer is a linear processor, no bias issues occur, and the output levels are quantitatively accurate. Of the two most critical aspects of this processing, the first, which is the number of modes that can be included in the beamformer at any particular frequency, has been fully resolved in Section $\mathbf{5 . 1 0}$ (see 
Table 5-1). The other is the estimation of an effective array tilt angle for each of the different signals recorded on the vertical array, the practical details of which are discussed at some length here.

Three different forms of output have been generated for each signal in the vertical array data set. One is a plot of the various modal amplitude estimates versus time for any assumed tilt angle. The second is a plot of the modal amplitude estimates at one particular time versus a range of assumed tilt angles. These two outputs can be thought of as being orthogonal cross-sections of a three-dimensional amplitude function, the axes representing time, mode number, and array tilt. This is illustrated in Figure 7-8. The third orthogonal cross-section would typically be a plot of the amplitude of one particular mode versus both time and array tilt. Instead, a somewhat more useful output is presented here to convey information in the third dimension. That output is the beamformer's residual error versus time and assumed tilt angle. This parameter is more appropriate because it may be considered to represent a projection or an integration of the amplitude data from all the modes into a single value which can then be plotted on the time-tilt surface. It is this integration effect across all the modes included in the beamformer that allows the residual error plot to provide direct information concerning the tilt of the array versus time.

\subsubsection{The Sensitivity of Vertical Array Data to Array Tilt}

The sensitivity of the modal decomposition process to vertical array tilt has been demonstrated theoretically and by simulation in Sections 6.2 and 6.3. Similar sensitivity is experienced when dealing with actual data, as seen in Figures $7-9$ and 7-10. Figure 7-9 shows a plot of the modal amplitude outputs from the multiple beam least squares beamformer plotted versus assumed array tilts between - 10 and 

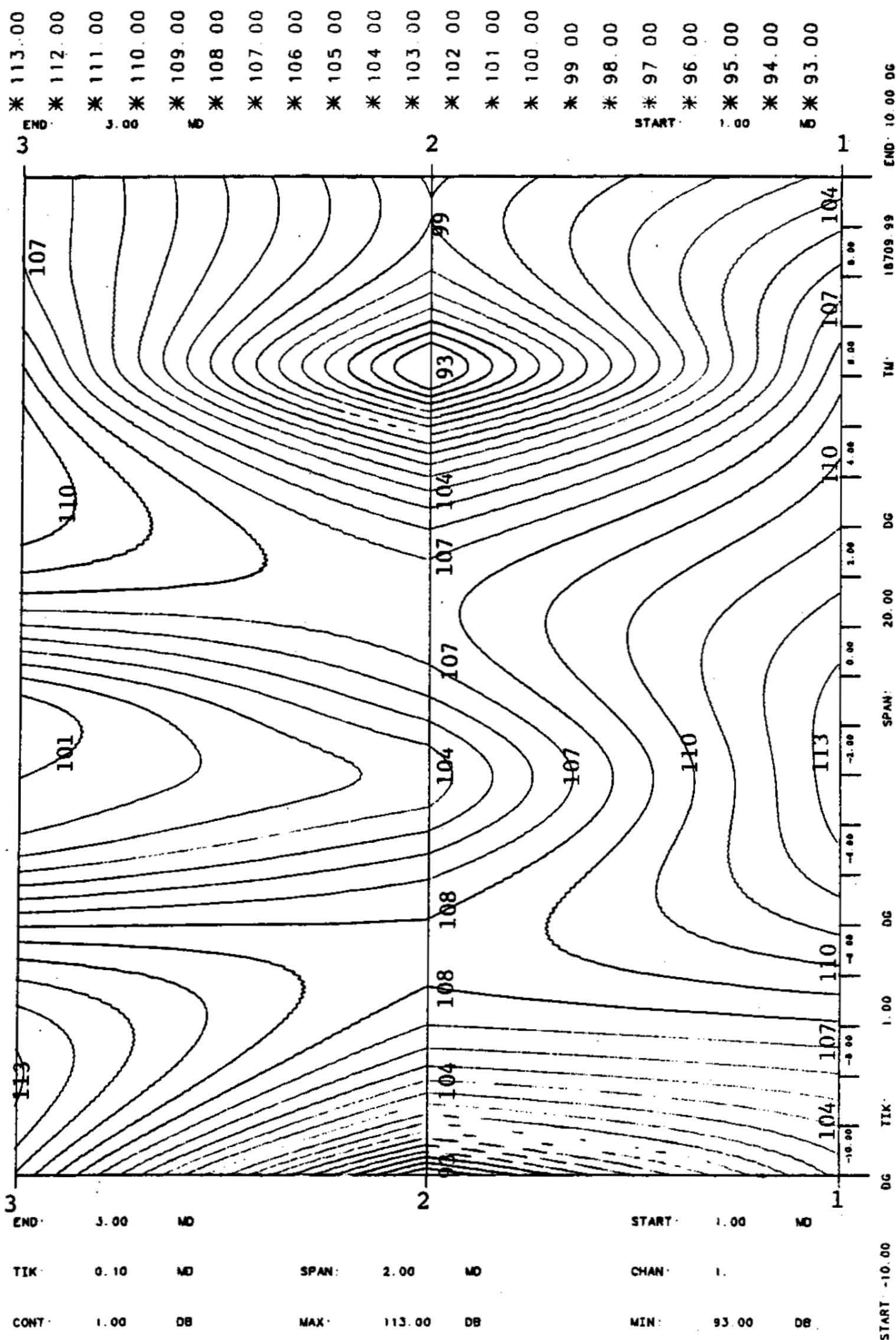

MODAL GEAMF ORMER ARRAY TILT SENSITIVITY AT $1775 \mathrm{HZ}$ (3 MOOES)

Figure 7-9: Output of Multiple Beam Least Squares Modal Beamformer versus Array Tilt for $17.75 \mathrm{~Hz}$ Tone (Vertical Array) 


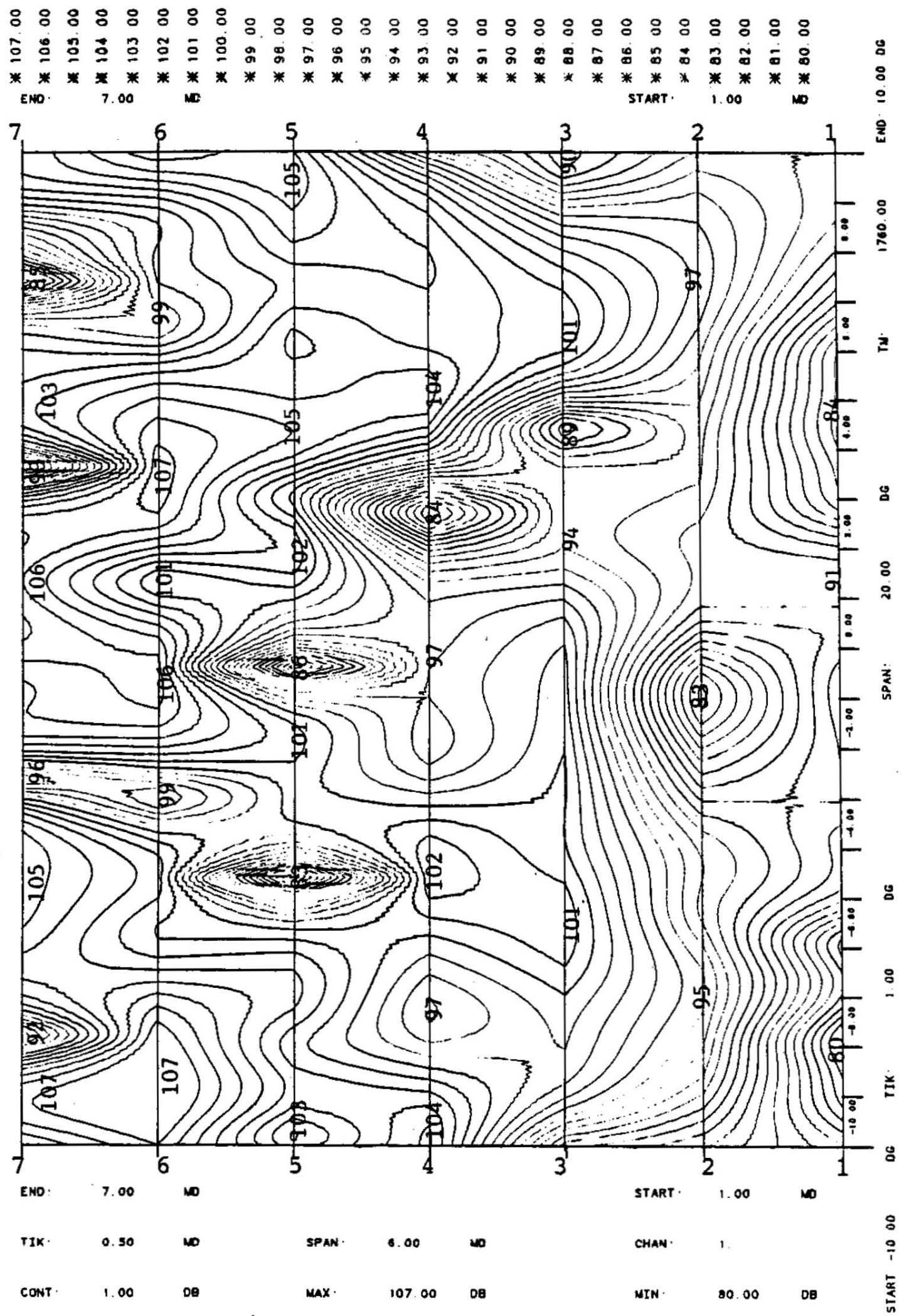

MODAL GEANFORMER ARRAY TILT SENSITIVITY AT 47:00 HZ (7 MODES)

Figure 7-10: Output of Multiple Beam Least Squares Modal Beamformer versus Array Tilt for $47.00 \mathrm{~Hz}$ Tone (Vertical Array) 
$10^{\circ}$. These results are computed from one particular $120 \mathrm{sec}$ window of data centered on the 18,710 sec mark in the signal time series (the amplitude and phase time series of this signal are displayed for the different vertical array hydrophones in Figures 3-11 and 3-12). The estimated modal amplitude levels for all thren resolvable modes are included on the plot. Even over just the range from $-5=$ to 5 (the most likely region for the actual effective tilt angle), significant variations in the modal amplitude estimates can be found. These variations are as much as $4 \mathrm{~dB}$ for mode 1 and $10 \mathrm{~dB}$ for mode 3 .

Figure 7-10 shows similar results for the $47 \mathrm{~Hz}$ tone from the vertical arrily data set; here, a total of seven modes can be resolved. The 2 minute window of data used for this figure is centered on the 1760 sec point of the signal time series (the time series for this signal can be found in Figures 3-9 and 3-10). The problem of tilt angle sensitivity obviously increases at higher frequencies; at any particular frequency, the estimates of higher order mode amplitudes appear to vary more with array tilt than do those of lower modes. Note the deep nulls displayed by some of the modes at selected tilt angles; variations in amplitude of more than $15 \mathrm{~dB}$ (an be seen over tilt variations of just $1^{\circ}$ in the $47 \mathrm{~Hz}$ result, for example. It is apparent that if one wishes to obtain accurate measurements of the various modal amplitudes, then estimates of the array tilt accurate to about \pm 0.25 " nust be available. At lower frequencies and lower mode numbers, this accuracy requirement may be relaxed somewhat. It is also important to observe that the sensitivity of amplitude measurements to assumed array tilt errors is quite dependent on both the mode being considered and the actua! tilt value; a $0.1^{\circ}$ change in array tilt is much more significant for some mode number/tilt angle combinations than for others.

Figures 7-9 and 7-10 do provide qualitative information about the various modal amplitudes, even without determining the actual array tilt. This information 
can be extracted through the gross averaging of results over all possible array tilts. The $17.75 \mathrm{~Hz}$ results indicate a strong first mode arrival relative to the second and third mode amplitudes; this is entirely consistent with previous conclusions for dat it at this frequency made in Sections 3.12 and 7.2. Similarly, the $47 \mathrm{~Hz}$ outputs of Figure 7-10 are in agreement with previous observations of a very low level amplitude for the first mode. This consistency generates confidence in both the operation of the beamformer and the validity of the observations.

\subsubsection{Determination of Vertical Array Tilt Angles}

Having demonstrated the sensitivity of the experimental results to the tilt of the vertical array, the next major step in processing the data is to make sufficiently accurate estimates of the array tilt at various times. If in situ measurements of both the magnitude of the tilt angle and its direction in azimuth were available. then they could be employed to resolve the issue directly. Since no nlch measurements are available for the FRAM IV Experiment, the alternative procedure outlined in Section 6.6 is employed here to make an estimate of the array till for each signal in the vertical array data set. This procedure involves minimization of the beamformer's residual error over the range of physically reasonable array tilts. Recall that the multiple beam least squares modal beamformer computes the full set of complex modal amplitudes which best fit the observed sound pressure field. given one assumed effective tilt angle. The normalized residual error is the performance measure for this fit (i.e., a measure of how good the fit really is). Even though the residual error is a scalar value, it should be remembered that its calculation is affected by the number of modes included in the multiple beam algorithm. By selecting the assumed tilt angle to minimize the residual error, the outputs generated by the beamformer then form the set of amplitudes that best fit the observed field for any possible array tilt. 


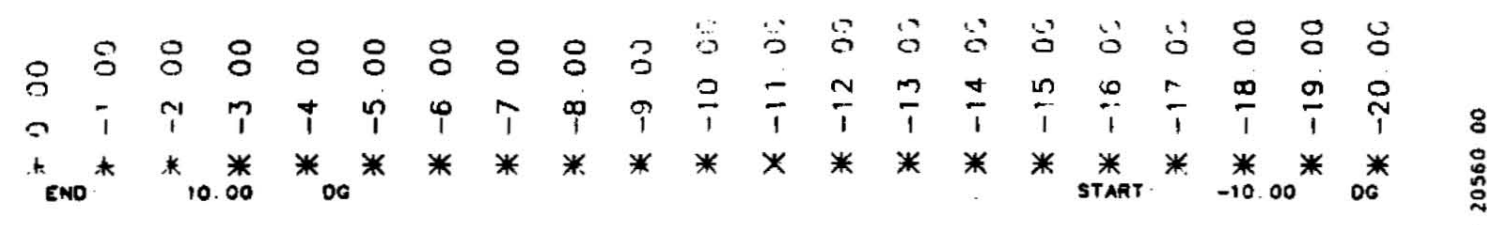

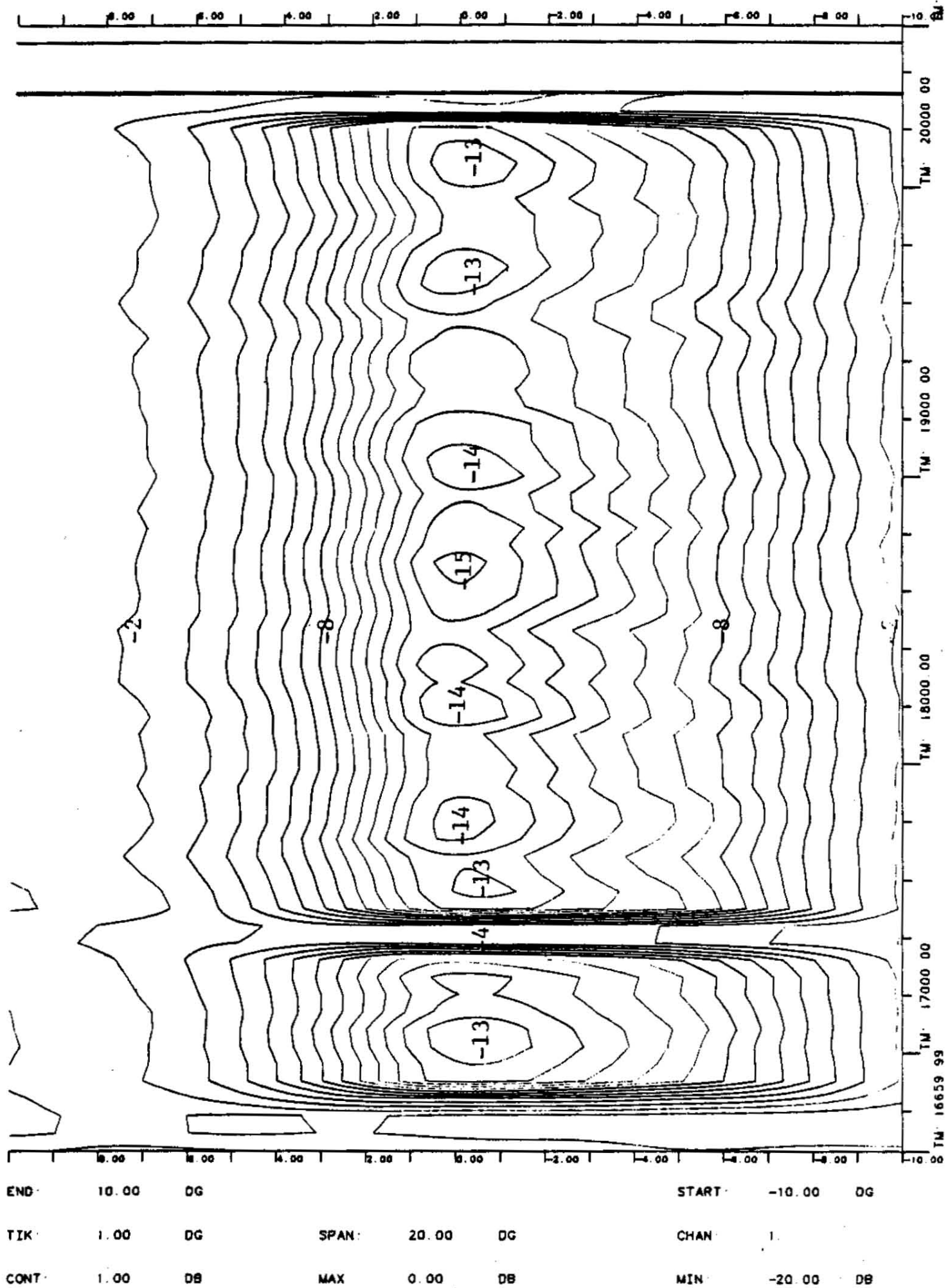

MODAL GEAMFORMER RESIDUAL ERROR AT $17.75 \mathrm{HZ}$ (3 MOOES)

Figure 7-11: Residual Error of Multiple Beam Least Squares Modal Beamformer versus Array Tilt for $17.75 \mathrm{~Hz}$ Tone (Vertical Arrav with 3 Modes Included) 


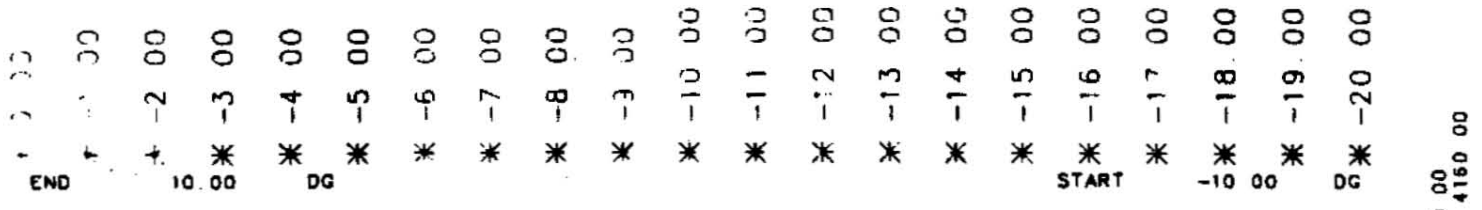

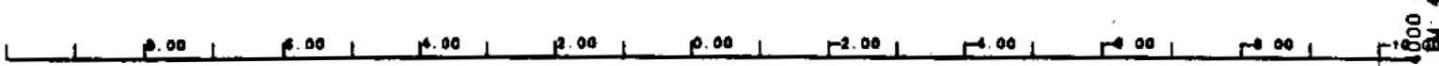

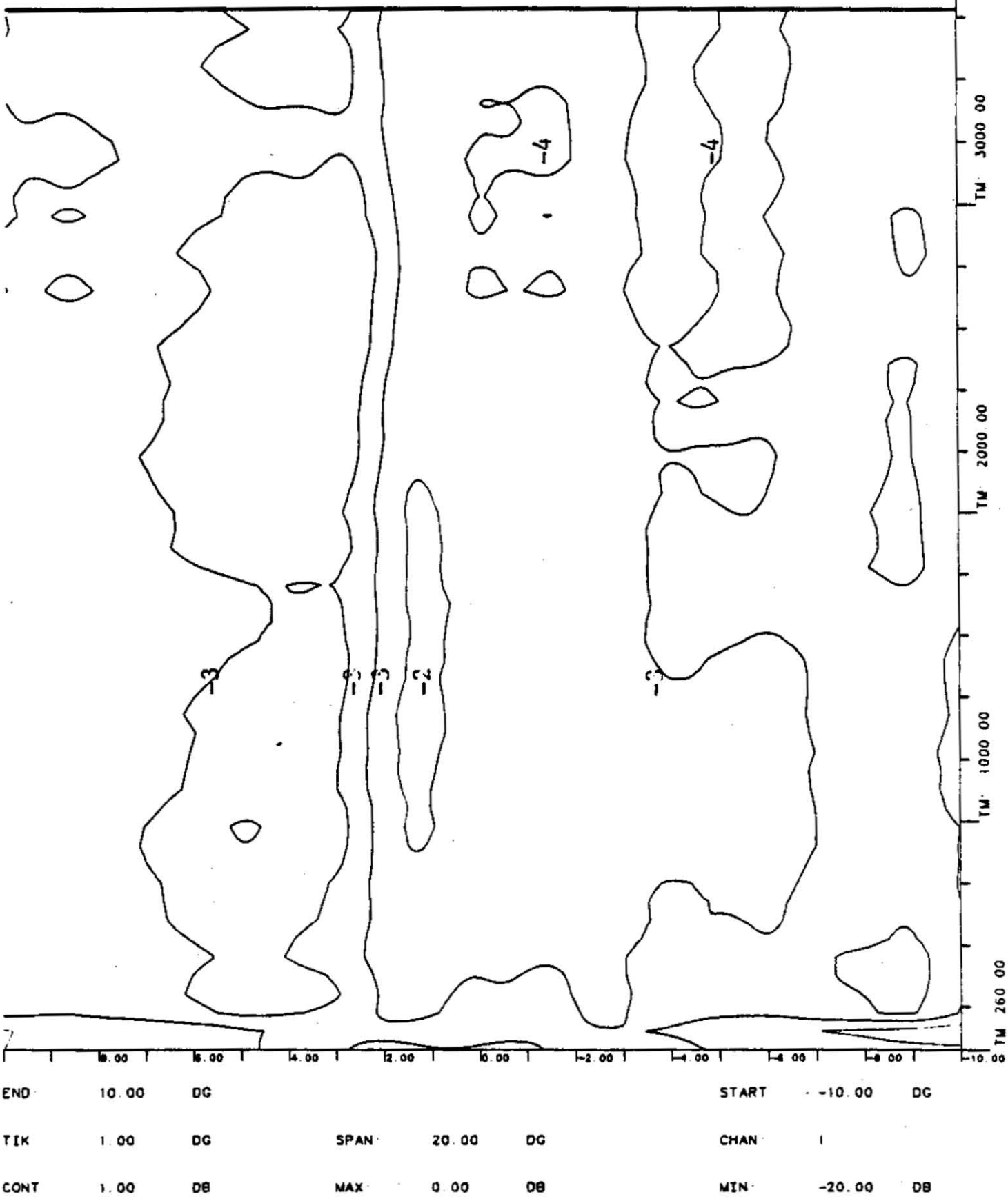

MOOAL GEAMFORMER RESIOUAL ERROR AT $4700 \mathrm{HZ}$ (7 MODES)

Figure 7-12: Residual Error of Multiple Beam Least Squares Modal Beamformer versus Array Tilt for $4 \bar{i} .00 \mathrm{~Hz}$ Tone (Vertical Array with 7 Modes Included) 
Indications exist that the array may not necessarily be completely stationary over periods of longer than about twenty minutes; the discussions in Sections 3.12 and 7.3.4 are germane. For the purposes of determining array tilt, however, any possible dynamic shifts in array shape over the signal's duration have been ignored, so that one average value of the array tilt can be computed for the full length of each signal. This is done primarily for ease in implementing the software necessary to perform the processing. A secondary consideration is that the tilt angle estimation scheme appears to lack the resolution necessary to accurately track much more than the largest variations.

Figure 7-11 shows a plot of the normalized residual error of the multiple beam least squares beamformer on the time-tilt surface for the $17.75 \mathrm{~Hz}$ data. Here, as in Figure $7-9$, the three modes resolvable by the array at $17.75 \mathrm{~Hz}$ have been included in the multiple beam algorithm. The error values have been computed at every $0.1^{\circ}$ of array tilt in the range from $-10^{\circ}$ to $10^{\circ}$. The array tilt generating the minimum residual error is $-0.15^{\circ}$. Data taken when the signal was off, as well as the data near the 17,400 sec point (when the airgun was active), have been excluded to avoid biasing the time average. The tilt that provides the minimum error is reasonably consistent over the length of the signal, and errors as small as $-15 \mathrm{~dB}$ (fitting $97 \%$ of the data) are achieved. The broad nature of the minimum presents some difficulty. While a determination of the minimum point can be made to any desired accuracy, some question exists as to the physical relevance of that accuracy. From the nature of Figure 7-11, it appears that the minimum tilt angle is probably known to better than $\pm 1^{\circ}$, but that the $0.25^{\circ}$ accuracy desired has not been achieved.

Figure 7-12 illustrates a similar set of results for the $47 \mathrm{~Hz}$ tone. The residual error computation now includes the seven modes resolvable at this higher frecunency. 
No value of array tilt in the expected range does a very good job of fitting the observed data; even the best fits account for less than $75 \%$ of the signal energy (equivalent to a $-6 \mathrm{~dB}$ error level). This result is believed to be due to the presence of significant amounts of signal energy in modes beyond the seven included in the beamformer. In the horizontal array data of Figure 7-6, such a distribution of energy in the higher modes has already been demonstrated; the preliminary analysis of Section 3.12 supports the same view. Further, in Section 6.6, it has been shown that such a distribution would effectively disrupt the ability of the scheme to properly estimate array tilts. Thus, the results of Figure 7-12 should be interpreted as proving that the first seven modes form an inadequate model for explaining the structure of the received field.

The two error plots just presented are typical of those generated by the remaining signals in the data set. In general, the received fields for signals below frequencies of about $40 \mathrm{~Hz}$ are adequately modeled by the modes that can be resolved with the FRAM IV vertical array; therefore, reasonable estimates of array tilt can be generated from the residual error analysis for these tones. Minimum residual errors of less than $-10 \mathrm{~dB}$ (better than a $90 \%$ fit) are typically achieved for these signals. The minimum can normally be determined to an accuracy of better than $\pm 1^{\circ}$ for these data sets, but the desired $0.25^{\circ}$ accuracy appears unachievable with this method. Based on Table 6-II, the technique probably provides accurate first mode results and partially accurate second mode results. The reliability of second and third mode amplitude estimates can be expected to improve somewhat at lower frequencies for two reasons: the sensitivity of the estimates to tilt angle decreases; and the residual error plots for these frequencies tend to provide better array tilt data.

By contrast, all of the signals above $40 \mathrm{~Hz}$ in frequency provide results similar 
to those shown in Figure 7-12, making even a rough estimate of array tilt impossible. Additionally, analysis of the $35.50 \mathrm{~Hz}$ signal, while producing a respectable minimum error, generates a double minimum several degrees apart. Therefore, no reliable tilt angle estimate can be made for this tone either.

The array tilts measured by the residual error minimization technique and the corresponding minimum errors achieved are listed in Table 7 -I. The best available fit generally becomes poorer with increasing frequency. The excellent fits achieved at the lowest end of the frequency spectrum must be at least partially attributed to the range dependence of the channel, which tends to suppress propagation of many of the modes that cannot be resolved by the array at the lower frequencies. Note that the tilt angles are reported to the nearest $0.05^{\circ}$ only because the beamforming algorithm requires an input of infinite precision.

\begin{tabular}{lccc} 
Frequency & $\begin{array}{c}\text { Minimum } \\
\text { Error }\end{array}$ & $\begin{array}{c}\text { Corresponding } \\
\text { Array Tilt }\end{array}$ & $\begin{array}{c}\text { Array Tilt } \\
\text { Assumed in } \\
\text { Processing }\end{array}$ \\
\hline $15.00 \mathrm{~Hz}$ & $-17 \mathrm{~dB}$ & $-70^{\circ}$ & $2.05^{\circ}$ \\
$17.75 \mathrm{~Hz}$ & $-14 \mathrm{~dB}$ & $-0.15^{\circ}$ & $-0.15^{\circ}$ \\
$20.00 \mathrm{~Hz}$ & $-12 \mathrm{~dB}$ & $2.95^{\circ}$ & $2.05^{\circ}$ \\
$23.50 \mathrm{~Hz}$ & $-10 \mathrm{~dB}$ & $2.70^{\circ}$ & $2.30^{\circ}$ \\
$30.00 \mathrm{~Hz}$ & $-11 \mathrm{~dB}$ & $1.40^{\circ}$ & $2.05^{\circ}$ \\
$35.25 \mathrm{~Hz}$ & $-12 \mathrm{~dB}$ & $-1.65^{\circ}$ & - \\
$35.50 \mathrm{~Hz}$ & $-9 \mathrm{~dB}$ & - & - \\
$47.00 \mathrm{~Hz}$ & $-4 \mathrm{~dB}$ & - & - \\
$53.25 \mathrm{~Hz}$ & $-4 \mathrm{~dB}$ & - & - \\
$55.00 \mathrm{~Hz}$ & $-5 \mathrm{~dB}$ & - & - \\
$71.00 \mathrm{~Hz}$ & $-7 \mathrm{~dB}$ & - & -
\end{tabular}

Table 7-I: Results of the Array Tilt Estimation Procedure

As mentioned above, the residual error analysis does not provide the tilt angle precisely enough to accurately estimate all of the modes that are resolvable by the vertical array. The tilt estimates may be refined further by comparing and 
averaging the angles estimated from tones broadcast consecutively. Such an effort also contributes by providing an independent means for checking the accuracy of the tilt angle estimation process. A review of Table 3-IV, for example, indicates that the 15,20 , and $30 \mathrm{~Hz}$ signals were all broadcast within one hour of each other. It is reasonable to expect that their array tilts should be approximately equal. This is in fact the case, with the largest difference between any pair of the three being only about $1.5^{\circ}$, implying an accuracy of roughly $0.8^{\circ}$ in these three tilt angle measurements. Assuming that the array did not move significantly over the course of the hour during which the three tones were broadcast, the accuracy of the tilt estimate can be improved by averaging the three individual findings. This yields a tilt angle estimate of about $2^{\circ}$, which is the value that has been used in the subsequent processing for all three signals.

The $17.75 \mathrm{~Hz}$ data was not taken concurrently with any other signal in the data set, so there is no way of verifying its estimated tilt angle. The $35.25 \mathrm{~Hz}$ data was taken just after the $23.50 \mathrm{~Hz}$ signal; a comparison between the array tilt estimates for these two cases is not very favorable, though, since a difference of over $4^{\circ}$ is found. Both residual error plots show minimum error tilt angles that are reasonably stable over the length of the signal. It is hardly likely that the array demonstrated a constant tilt for the $\mathbf{5 5}$ minute duration of the earlier signal. and then moved $4^{\circ}$ in tilt during the 5 minute pause between signals before once more becoming stationary for the full duration of the second signal. It is more reasonialble to suspect that at least one, and possibly both, of the estimates are being biased by signal energy in the unresolvable modes. Such an effect has been demonstrated with synthetic data in Figure 6-8. The horizontal array results of the previous section imply that the $35.25 \mathrm{~Hz}$ result is probably the less reliable of the two estimates. Therefore, no tilt angle has been assigned for subsequent processing of 
this tone. In consideration of the $35.25 \mathrm{~Hz}$ result, however, a small correction has also been made in the tilt angle assumed when processing the $23.5 \mathrm{~Hz}$ data.

\subsubsection{Time Series of the Modal Amplitudes}

Figure 7-13 displays the modal amplitudes as a function of time for the three modes resolvable by the array at $17.75 \mathrm{~Hz}$. These levels were computed assuming a tilt angle of $-0.15^{\circ}$, as indicated in Table 7-I. The results shown here are in agreement with the amplitudes shown in Figure $7-9$ for this tilt angle. The amplitude of the first mode is about $113 \mathrm{~dB}$ re $1 \mu \mathrm{Pa}$; the second mode amplitude is $7 \mathrm{~dB}$ below this. The relative amplitudes of the first and second modes thus agree with predicted values, assuming that both modes are attenuated equally. The amplitude of the third mode when compared with the first two is somewhat more problematic. The measured amplitude is $5 \mathrm{~dB}$ lower than that of the second mode. while approximately equal levels are expected. This error can best be explained by referring back to Figure $7-9$. At an assumed tilt angle of $-0.15^{\circ}$, the third mode estimate lies in a region of high sensitivity to tilt angle, so that even small changes in the assumed array tilt can greatly alter the beamformer output. These r(mults support the earlier conclusion that at these frequencies, the array tilt is known wefl enough to accurately determine at least the first two modes.

Only about $1 \mathrm{~dB}$ of fluctuation is seen in Figure $7-13$ for both the first and second mode estimates over the 55 minute duration of the signal (not including the period of the airgun blast). These levels are in agreement with those seen in the horizontal array data. The seemingly large contour variations over time are due to the fact that only three data points are represented along the vertical axis. Fluctuations in the third mode amplitude are only a little larger, perhilps 2 d lB. This observation provides a strong indication that the array was essentially 


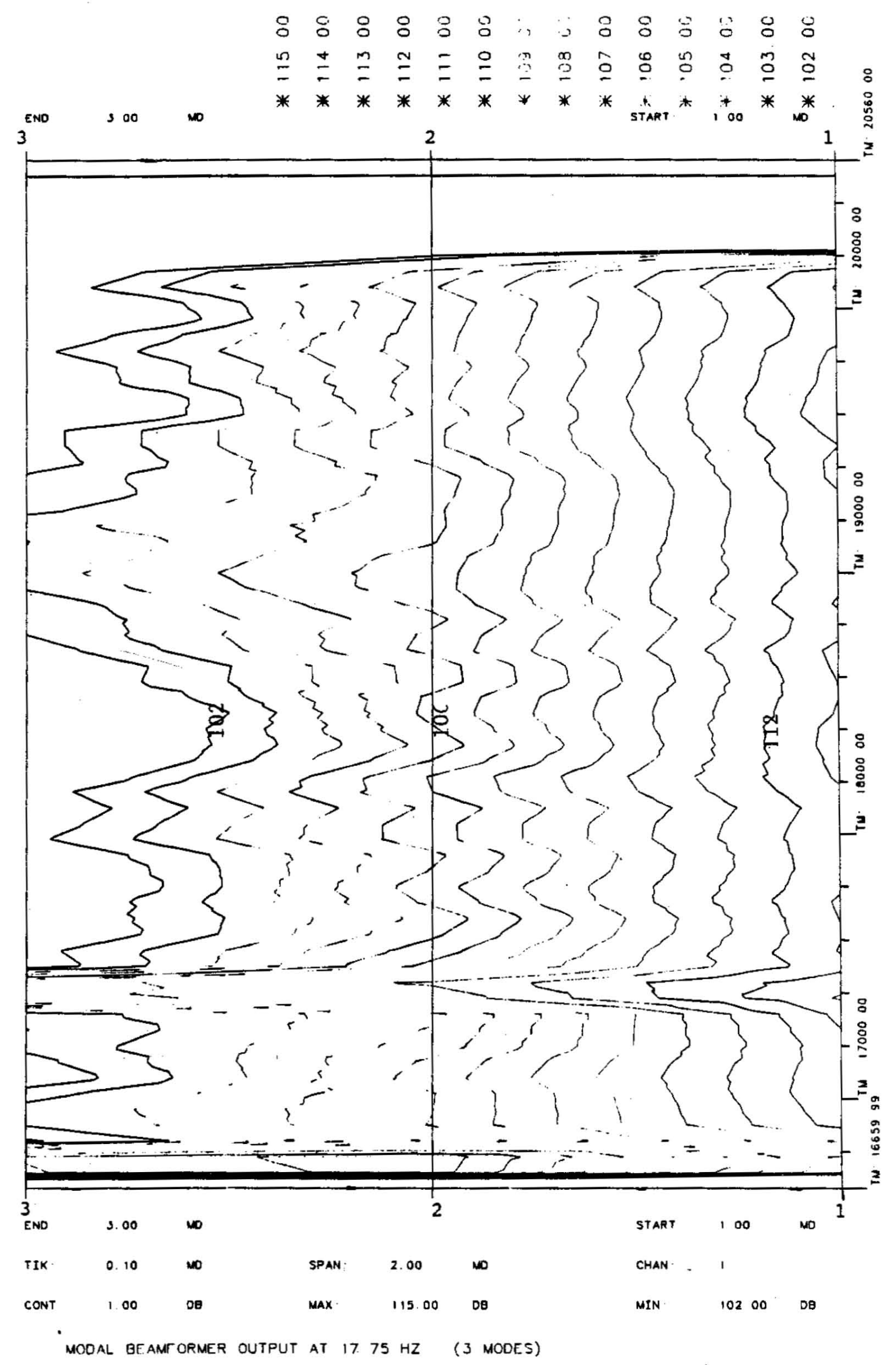

Figure 7-13: Output of Multiple Beam Least Squares Beamformer versus Time for $17.75 \mathrm{~Hz}$ Tone (Vertical Array) 
stationary for the duration of the signal, since even small amounts of array movement would generate large fluctuations in the highly sensitive third mode estimate.

For the signals with frequencies of $30 \mathrm{~Hz}$ and below, reasonably reliable array tilt estimates have been made; hence, reliable time series of the modal amplitudes can be obtained. All the time series show a dominant first mode structure, which is in general agreement with the horizontal array results. The fluctuation levels observed in these time series are also often comparable to the levels seen in the horizontal array data. In certain instances, more significant fluctuation levels are observed. The time scales associated with these larger fluctuations are almost always larger than 10 or 20 minutes. Since it is hypothesized that the cause of these variations is vertical array movement, they are taken up in the following section.

Since the array tilt cannot be determined for the vertical array signals with frequencies greater than $30 \mathrm{~Hz}$, valid time series results cannot be generated. The best that can be done is to assign a number of different tilt angles on a provisional basis and then to examine the results, realizing that the absolute modal amplitudes are inaccurate, but possibly allowing some general conclusions to be drawn. particularly about signal fluctuations over time. The modal time series getnerilted by this procedure show a pattern similar to that seen at lower freculuencies. Some signals show only very little fluctuation, and when larger variation is observed over time, it is invariably associated with the time scales of longer than 10 minutes. These also are discussed in the following section.

\subsubsection{Possible Array Movements Seen in the Vertical Array Data}

Several of the signals below $30 \mathrm{~Hz}$ have indications of possible array movement over the duration of the signal. One such example is the $23.5 \mathrm{~Hz}$ tont. 


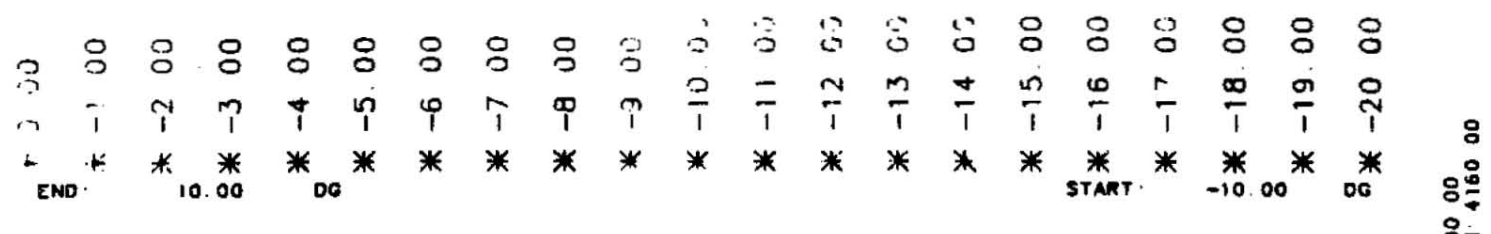

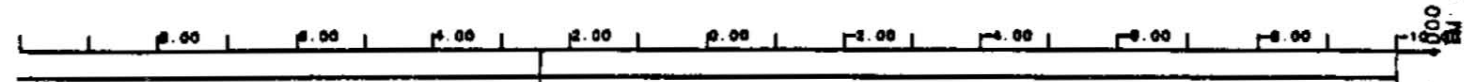

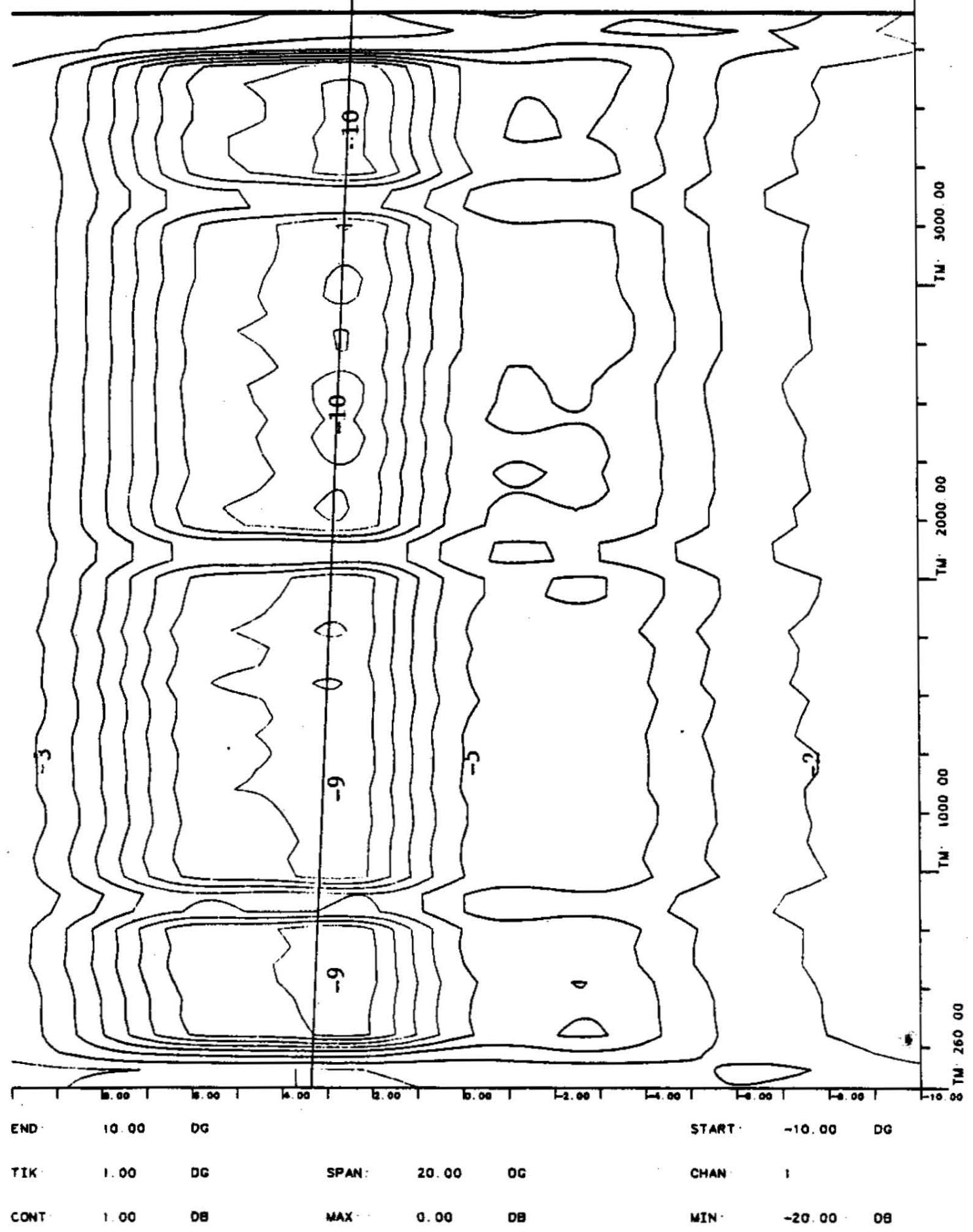

MODAL BEAMF ORMER RESIDUAL ERROR AT 23.5O HZ (4 MODES)

Figure 7-14: Residual Error of Multiple Beam Least Squares Modal Beamformer versus Array Tilt for $23.50 \mathrm{~Hz}$ Tone

(Vertical Array with 4 Modes Included) 


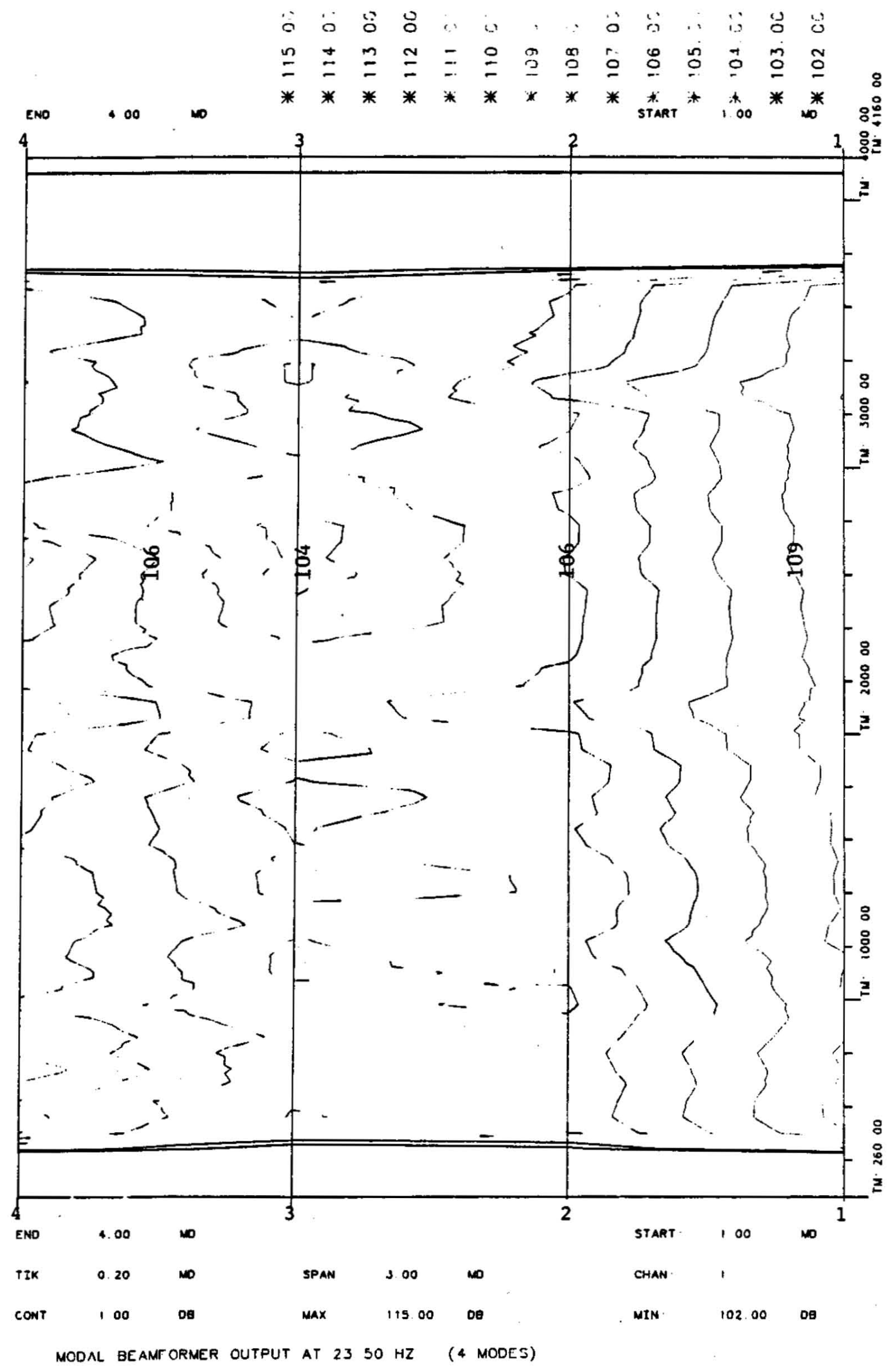

Figure 7-15: Output of Multiple Beam Least Squares Beamformer versus Time for $23.50 \mathrm{~Hz}$ Tone (Vertical Array) 
The residual error plot for this signal is presented in Figure $\mathbf{7}-14$, while Figure $7-15$ provides the modal amplitude time series data for the signal for an assumed array tilt of $2.30^{\circ}$. The amplitude time series show a small (but consistent) trend over time; the first and second mode amplitudes tend to increase slightly, while the third mode amplitude decreases. This drift is also reflected by an equivalent trend in the residual error data, which shows about a $1^{\circ}$ change in the minimum error tilt angle over the hour duration of the signal. These variations may be due to changes in the array tilt over time, or they may be actual fluctuations caused by changes in the acoustic transmission path. Unless actual array tilt measurements are available, the two effects are fundamentally inseparable. Certainly, the data presented here does not preclude either explanation. However, the consistency of the residual error result over time suggests that at least some of the variations observed here are due to array movement during the signal. It is important to observe that the variations under discussion appear to have time scales in the 10 to 20 minute range, and that there is no evidence of significant fluctuations possessing significantly shorter time scales.

Although it is not possible to obtain accurate modal amplitude results in an absolute sense for the signals that are above $30 \mathrm{~Hz}$ in frequency, the stability of these signals over time can be assessed in a qualitative fashion by computing the modal decomposition for an arbitrary tilt angle. For such i situation, the outputs of the beamformer can be expected to be constant over time if no significant temporal fluctuations are present in the sound field. When this is done, many of the signals above $30 \mathrm{~Hz}$ demonstrate a considerable amount of long term variation in the resultant modal amplitudes. It is even more difficult to assess the meaning of these long term fluctuations than it is for those present in the lower frequency data, since meaningful residual error analysis is not available. 
In summarizing the issues of fluctuations and array movement in the modial amplitude time series generated by the vertical array, it can be said that the short term amplitude fluctuations observed (those with time scales on the order of minutes) are small, and roughly equivalent in size to those seen in the horizontal array data. Some longer term fluctuations, of time scales that are greater than 10 or 20 minutes, have been observed in the vertical array data. A definitive separation of these variations into array movement effects and propagation path fluctuation effects is not possible, due to the lack of field measurements of vertical array tilt angle over time. Two observations provide circumstantial evidence that at least the fluctuations in the lower frequency data (that at $30 \mathrm{~Hz}$ and below) may be primarily related to array movement. The first is the nature of the residual error analyses available for the low frequency data; these analyses are consistent with the array movement explanation. The other is the lack of any similar fluctuations in the processed outputs of the horizontal array data, which indicate that the phenomena are probably array specific. Long term fluctuations in vertical array modal amplitudes for frequencies greater than $30 \mathrm{~Hz}$ are similar to those below $30 \mathrm{~Hz}$; reliable residual error analyses and comparative horizontal array data are lacking for these signals, though.

\subsection{Modal Amplitude Analysis}

The absolute modal amplitude estimates available from the beamformers can be exploited in two ways. The values generated from the vertical array data by modal beamforming can be compared to those made with more standard plane wave techniques employed with the horizontal array data in order to quantitatively verify the consistency of the two methods. This must be accomplished with first mode data, since the horizontal array cannot provide accurate amplitude estimates for 
higher order modes. Also, the comparison of observed levels to those predicted from propagation theory provides insight into the unique nature of long range propagation of low frequency sound in the central Arctic, and allows experimental measurements of modal attenuation coefficients to be made.

\subsubsection{Comparison of First Mode Amplitude Estimates from the Horizontal and Vertical Arrays}

Figure 7-16 summarizes the estimates of the first mode amplitudes versus frequency for both the horizontal and vertical array data sets. When the two groups of results are compared, it can be seen that the horizontal array values uniformly exceed the vertical array levels by about $4 \mathrm{~dB}$ over the 15 to $30 \mathrm{~Hz}$ range. the region in which both sets of estimates are most accurate. The lack of accuratt array tilt estimates make the vertical array results outside this range less reliable: the values shown for the horizontal array at 47 and $53.25 \mathrm{~Hz}$ are also questionable, since the first mode no longer dominates in these signals. For this reason, the data points at these frequencies have been connected with a dotted line. The $4 \mathrm{~dB}$ difference between the two sets of results appears to have two causes. A slight mismatch between horizontal and vertical array hydrophone sensitivities accounts for about 1 or $2 \mathrm{~dB}$ of the difference. The remaining $2 \mathrm{~dB}$ error is due to the slightly different propagation paths over which the two sets of signals were transmitted. Although the signals in each data set were grouped fairly closely in time, eight days elapsed between the last horizontal array data and the first vertical array data studied here. Note, however, that the issue is not merely one of range. as the TRISTEN/FRAM range for the horizontal array data $(26.5 \mathrm{~km})$ was actually slightly larger than that for the lower level vertical array data $(254 \mathrm{~km})$. 


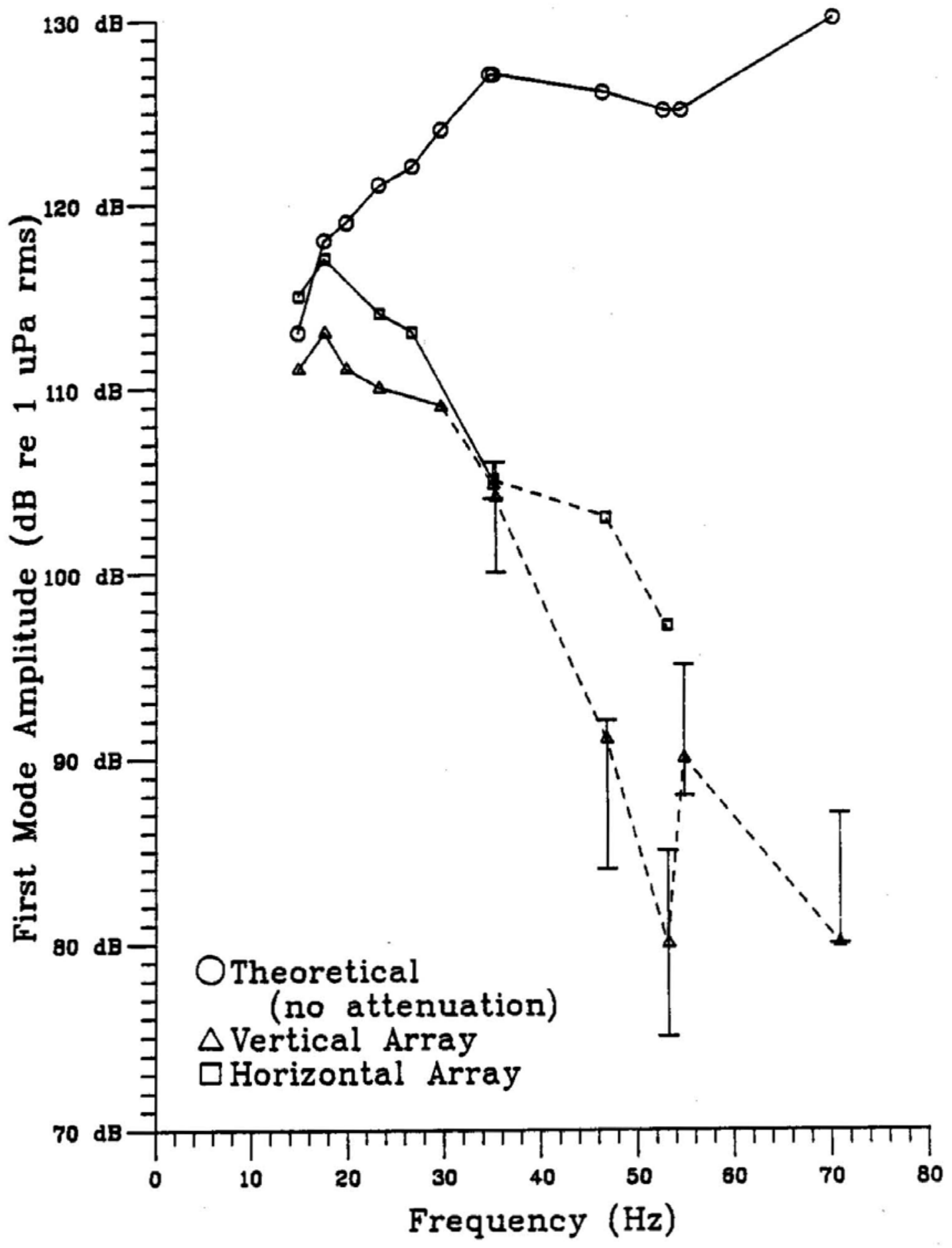

Figure 7-16: Summary of First Mode Amplitude Measurements 


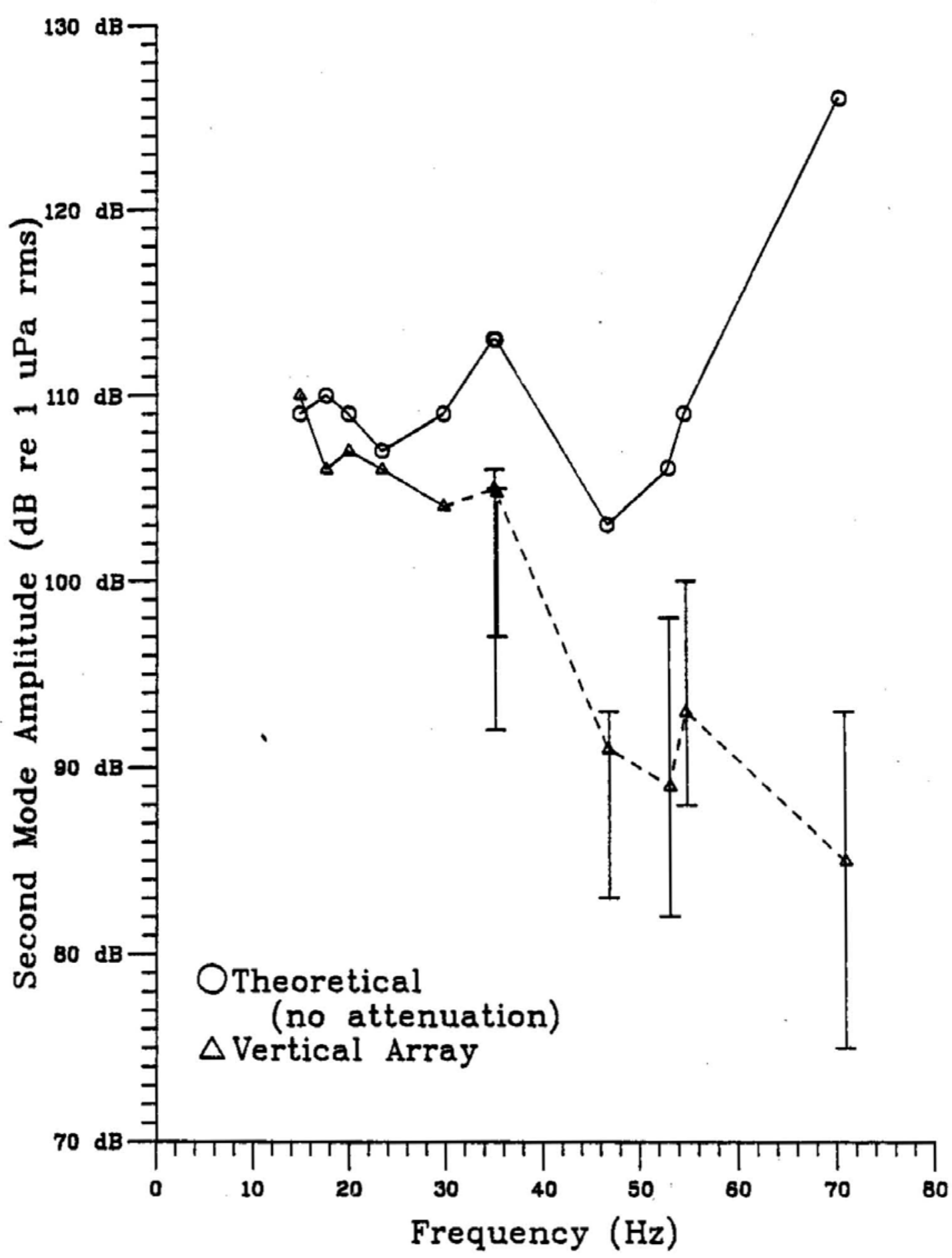

Figure 7-17: Summary of Second Mode Amplitude Measurements 


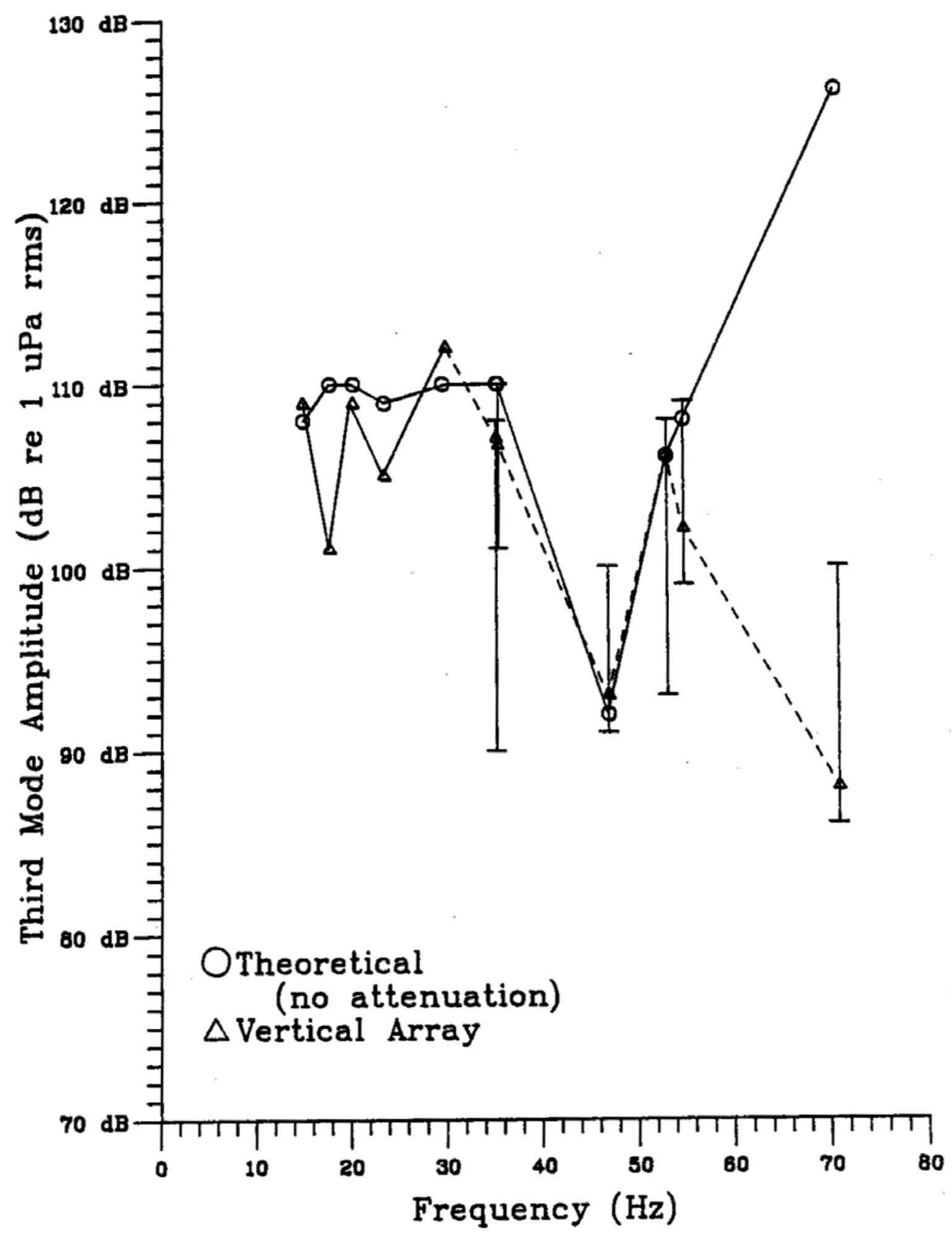

Figure 7-18: Summary of Third Mode Amplitude Measurements 


\subsubsection{Modal Attenuation Coefficient Measurements}

In addition to the data described above, Figure 7-16 also shows two other types of information. The amplitudes of the first mode estimated from vertical array data at higher frequencies are presented in a different fashion than those at lower frequencies. Since no accurate estimate of array tilt is available at these frequencies, the range of amplitudes generated by all possible array tilts between $-4^{\circ}$ and $4^{\circ}$ is displayed instead. The actual markers that are connected by the dotted line indicate the first mode levels for one subjective estimate of the actual array tilt. While this data is much less accurate than the lower frequency results, the ranges do provide valuable information about the general trend of the amplitude with frequency.

Also presented is the amplitude of the first mode as a function of frequency at a range of $254 \mathrm{~km}$ for an ideal horizontally stratified channel (i.e., geometric spreading with no attenuation mechanisms present). Figure 3-3 has been used as the source spectrum in computing these levels. The difference between this cirve and the experimental results indicates the attenuation loss for the first mode as a function of frequency. It is obvious that an attenuation mechanism is at work in the sound channel, and that it gets quite severe at higher frequencies. The attenuation is the cause of the unexpectedly low first mode amplitudes seen above $40 \mathrm{~Hz}$ in the data.

Figures 7-17 and 7-18 summarize similar results for the second and third modes, respectively. Again, the effect of the attenuation mechanism can be seen, particularly at the higher frequencies. Note that the measured modal amplitudes for the higher modes display greater scatter than do those for the first mode; this is indicative of the increased sensitivity to array tilt errors that the modal beamforming process exhibits at higher order modes. 


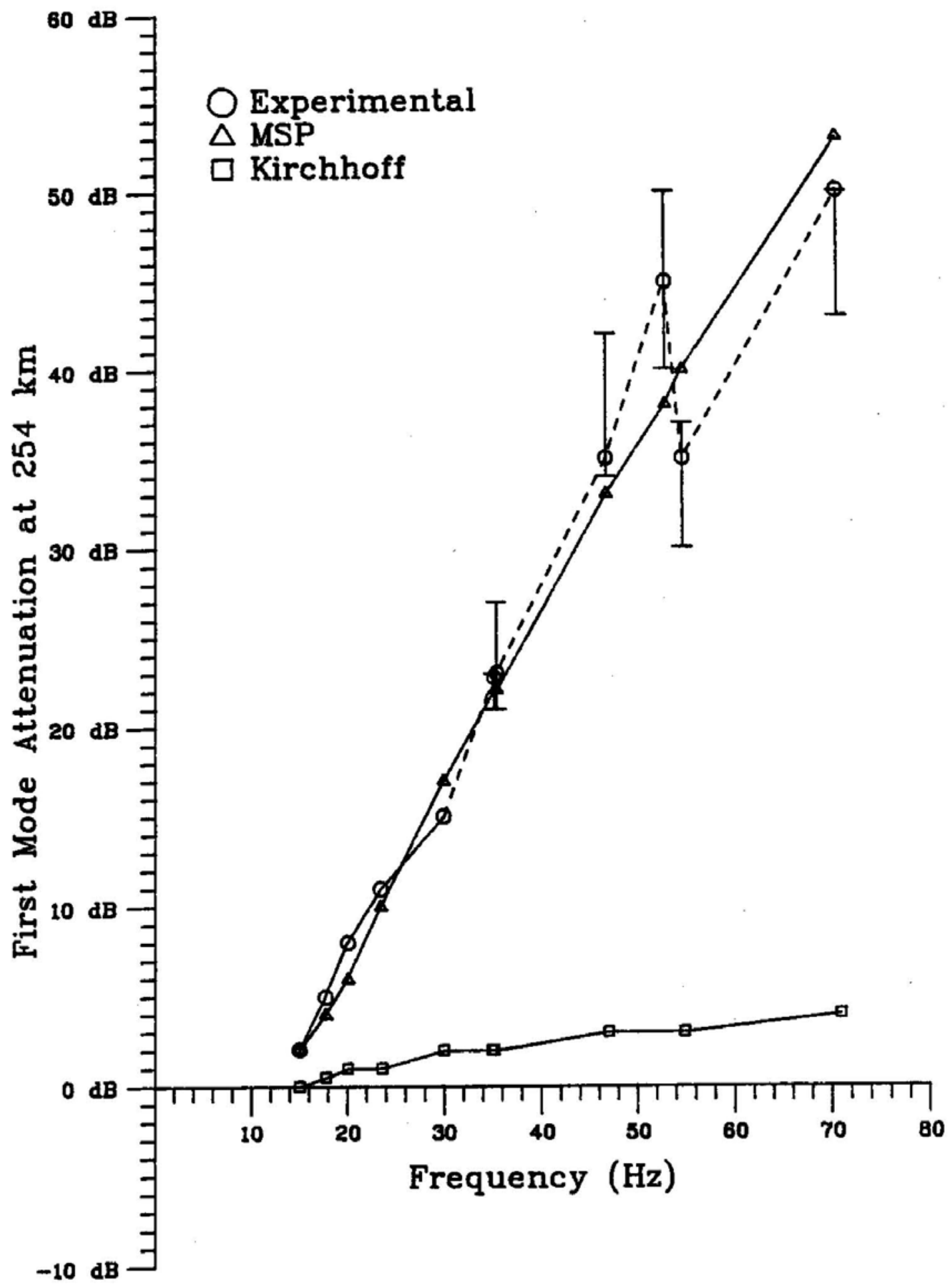

Figure 7-19: First Mode Attenuation - Experimental and Predicted 


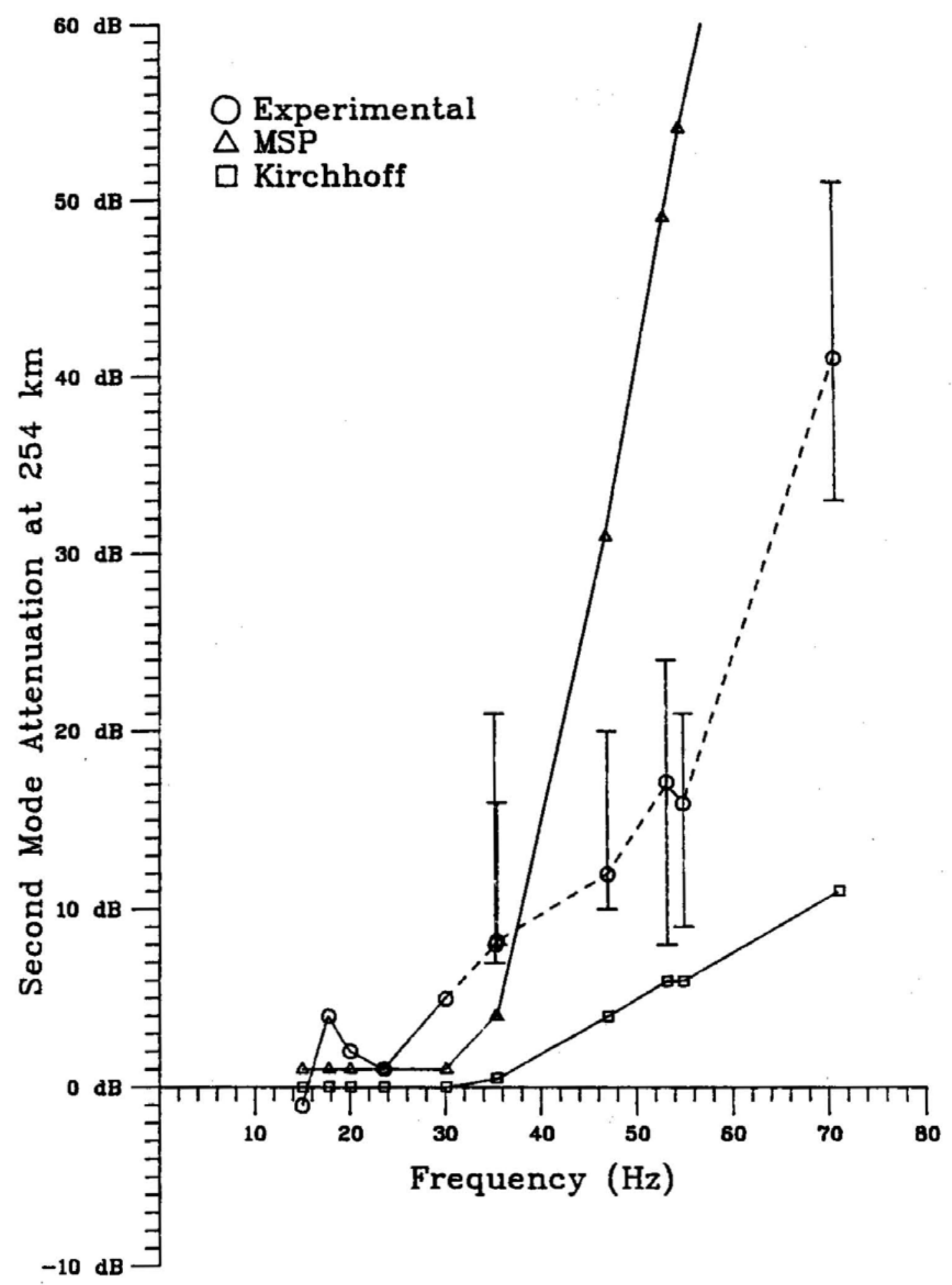

Figure 7-20: Second Mode Attenuation - Experimental and Predicted 


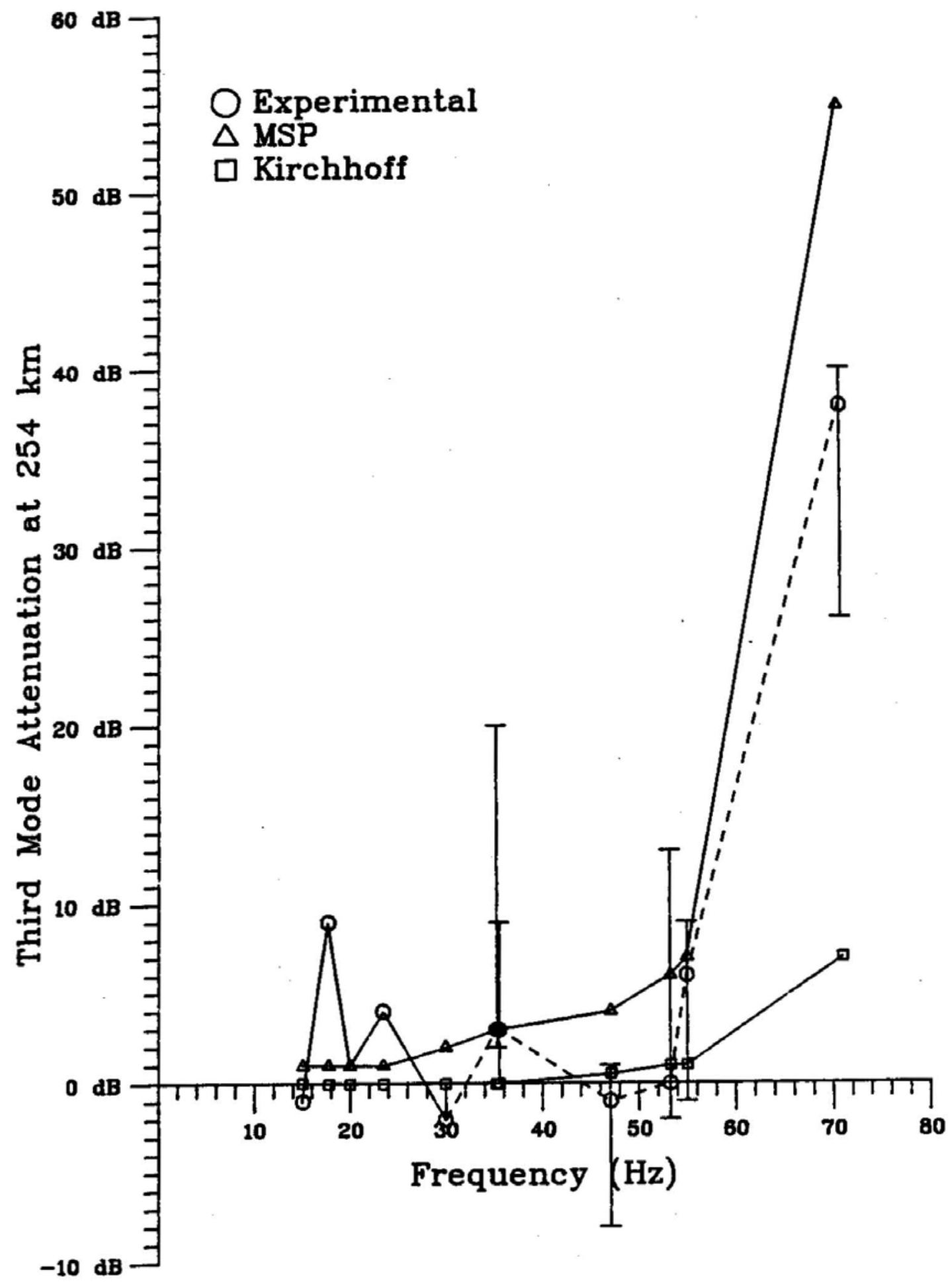

Figure 7-21: Third Mode Attenuation - Experimental and Predicted 
To characterize the modal attenuation further, Figures 7-19, 7-20, and $7-21$ have been included. In these plots, the actual attenuation experienced by the first, second, and third modes is displayed as a function of frequency. Vertical array results at higher frequencies are again plotted as ranges connected by a dotted line. The data is plotted on a scale of total attenuation at $254 \mathrm{~km}$; equivalent attenuation coefficients can be recovered simply by dividing the $\mathrm{dB}$ levels by this range.

Experience [25] suggests that the cause of the attenuation is the rough ice canopy; a comparison of the three plots supports this supposition by demonstrating directly that the attenuation mechanism is located in the surface duct of the central Arctic profile. Such a conclusion can be reached by noting the relationship between the onset of severe attenuation and the mode number. In Figure $7-19$, the experimentally determined attenuation for the first mode is seen to be strongly increasing, even at the lowest frequencies in the data set. By comparison, the point where attenuation becomes significant is delayed somewhat in frequency for the second mode (Figure 7-20), and even more for the third mode (Figure 7-21). Typical frequencies for the onset of severe attenuation are $30 \mathrm{~Hz}$ for the second mode and $50 \mathrm{~Hz}$ for the third mode. These values correlate well with the frequencies at which the various modes transition into the surface duct, as discussed in Section 4.5.2. From this, one can conclude that the attenuation mechanism must be located in the surface duct, making the rough ice surface a prime candidate.

Figures 7-19 through 7-21 also include plots of predicted modal attenuation versus frequency for two different rough surface scattering theories. Both rases assume a rough pressure release boundary at the ice-water interface. The statistics of this boundary are assumed to be those given by DiNapoli and Mellen [25], as discussed in Section 4.3. The simpler case is the well known Kirchhoff approximation, for which the attenuation coefficient of the $i^{\text {th }}$ mode can be 
computed from

$$
\alpha_{i}=\frac{\sigma^{2} \phi_{i}^{2}(0)}{2 \rho_{0}} \sqrt{\left(\frac{c_{i}}{c_{0}(0)}\right)^{2}-1} .
$$

Here $\sigma$ is the surface roughness (taken to be $2 \mathrm{~m}$ ) and $c_{0}(0)$ is the sound speed at the surface, while $\phi_{i}^{\prime}(0)$ is the slope of the mode's normalized shape function evaluated at the surface and $c_{i}$ is its horizontal phase speed. The other case is the full method of small perturbations (MSP) solution, as developed by Kuperman and Ingenito [-17]. In this development, the modal attenuation is shown to be

$$
\alpha_{i}=\frac{\sigma^{2} \phi_{i}^{\prime 2}(0)}{2 \rho_{0}} \operatorname{Re}\left[A\left(c_{i}\right)\right],
$$

where

$$
A\left(c_{i}\right)=\frac{c_{i}}{(2 \pi)^{2} f_{0}} \int \sqrt{\left(\frac{2 \pi f_{0}}{c_{0}(0)}\right)^{2}-|\vec{\eta}|^{2}} P\left(\frac{2 \pi f_{0}}{c_{i}} \vec{u}-\vec{\eta}\right) d \vec{\eta}
$$

In equation (7.3), $P(\vec{x})$ is the two-dimensional spectrum of the rough surface there given by equation (4.1) with an assumed correlation length of $44.8 \mathrm{~m}$ ), and $\vec{u}$ is a unit vector in the direction of propagation. The integration is carried out over a full range of vectors $\vec{\eta}$ in the horizontal plane. Details of the derivations of both these results can be found in [47]. It is important to note that both contain factors that include the square of the slope of the mode shape evaluated at the surface, a term which is not present in the more familiar plane wave scattering results, and, hence. often overlooked. These factors reflect the ability of the ice to scatter modes with their energy concentrated near the surface more strongly than those with their energy concentrated at deeper depths.

The attenuation predictions of both models for the first three modes have been plotted in Figures $7-19$ through $7-21$, respectively. The agreement of the 
experimental data with the Kirchhoff approximation is uniformly poor. On the other hand, the attenuation results predicted by the MSP match the first mode data quite well, and the second mode data somewhat less exactly. It is possible to speculate that the poorer second mode fit is caused by the scattering of first mode energy into the second mode by the ice. In general, any scattered energy redistributes itself across the modal spectrum. If significant amounts of motion were present at the surface, the scattered energy could be expected to be temporally incoherent when compared with the transmitted signal. For the Arctic, though, it appears reasonable to model the rough surface as being essentially stationary over time, so that an scattered energy would still be coherent with the specular transmission. Much of the scattered energy is lost into higher order modes that do not propagate well in the channel; however, a considerable percentage can be expected to end up in the lower order modes. In fact, the second mode will normally receive the largest share, since modal coupling is typically strongest between neighboring modes. In the 30 to $60 \mathrm{~Hz}$ frequency band, the first mode excitation exceeds that of other modes by 15 to $30 \mathrm{~dB}$ (see Figure 4-9). Thus. energy scattered from the first mode into the second mode may be significant when compared to second mode excitation levels, even though the coupling coefficient itself is negligibly small. Under such circumstances, the attenuation experienced by the second mode would appear to be reduced, as is the case in Figure $\mathbf{i}-20$.

The MSP also appears to fit the third mode data (Figure $i-21)$ reasonably well, although there are hints of a phenomenon similar to that of the second mode in the $71 \mathrm{~Hz}$ data point as well. It must be remembered that the third mode dat a is probably only partially resolved at best, due to the inability to estimate the array tilt angle to sufficient accuracy. As might be expected, the third mode data display less consistency than those of the other two modes. 


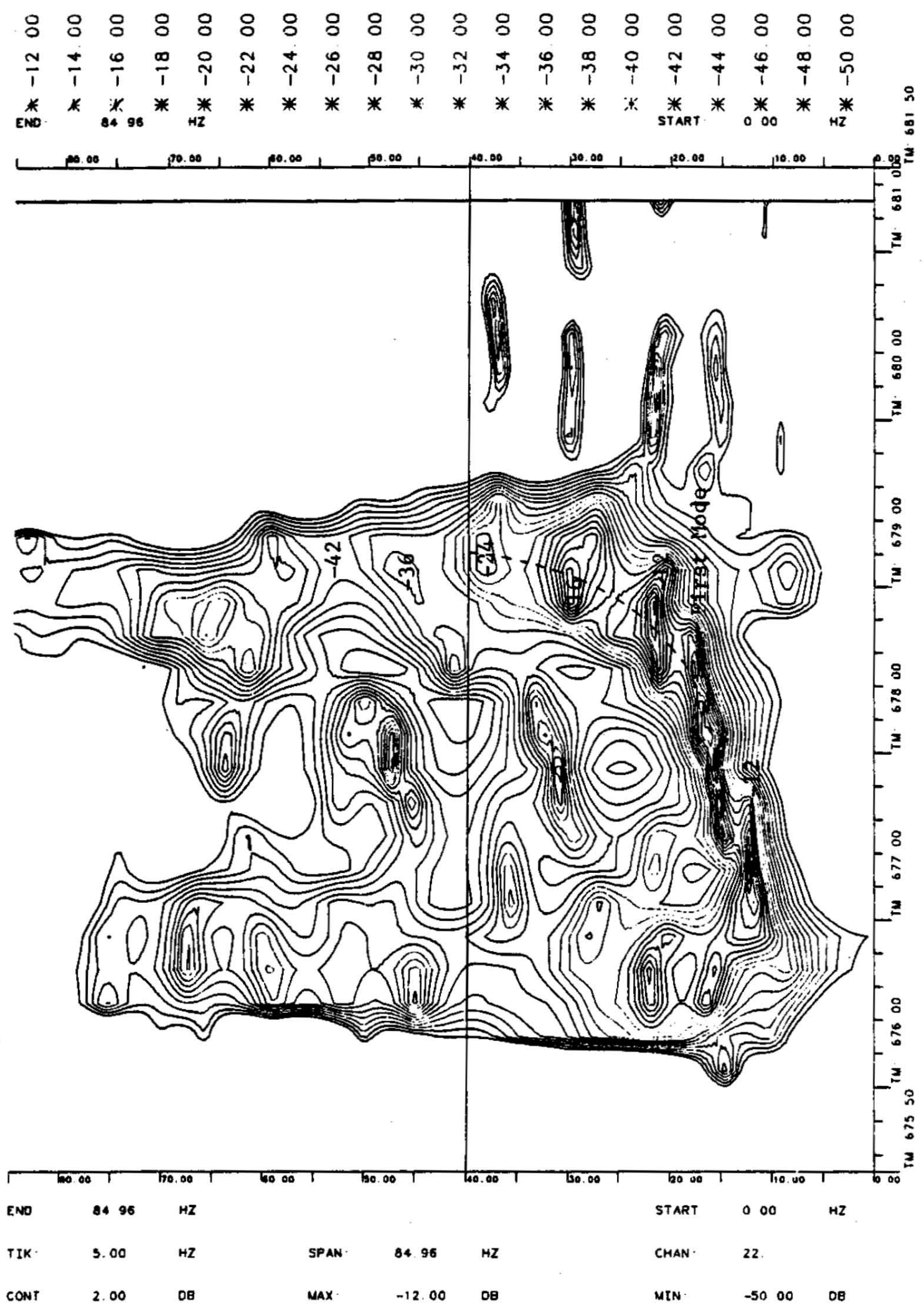

SONOGRAM OF SENSOR AT $90 \mathrm{M}$ FOR SHOT TRANSMITTED OVER TRISTEN/FRAM CHANNEL

Figure 7-22: Sonogram of Explosive Shot Transmitted Over the TRISTEN/FRAM Channel as Received on $90 \mathrm{~m}$ Hydrophone of the Vertical Array (300 ft Source Depth) 


\subsubsection{Comparison with Shot Data}

It is of interest to contrast the horizontal and vertical array data results just presented with data taken from an impulsive source broadcast over the same channel. This exercise confirms the horizontal array modal received level measurements directly and the vertical array results indirectly. It also generates insight into the relationship between the results of two data sets.

Figure 7-22 displays the sonogram of a shot set off at 300 feet and then transmitted over the TRISTEN/FRAM sound channel. The sonogram is a plot of received level versus frequency and time, and is generated by computing a sequence of very short length spectral estimates from the time series output from a single hydrophone, in this case the $90 \mathrm{~m}$ hydrophone from the vertical array. The results shown here were generated using a Burg spectral estimation technique to provide

greater frequency resolution in the $250 \mathrm{msec}$ window length used. A new spectral estimate was completed every $100 \mathrm{msec}$. The original time series for this shot has already been presented in Figure 1-2.

The peak marked with a dotted line is the first mode arrival, corresponding to the long tail of the signal displayed in Figure 1-2. This can be shown from a comparison of the measured dispersion characteristic with that expected of the first mode. Note that the received level of the first mode arrival is quite strong and is reasonably flat versus frequency up to about $40 \mathrm{~Hz}$, after which it almost completely disappears. This is exactly the effect that has been demonstrated in the horizontal array tonal data which, in turn, have been shown to be in quantitative agreement with the vertical array tonal results in Figure 7-16. In retrospect, the strong drop in the received level of the first mode in both the horizontal array data and the shot data can be seen to be a combination of two effects. The first mode exhibits some attenuation even at the lowest frequencies in the data set, and this attenuation 
increases rapidly at higher frequencies. Below $40 \mathrm{~Hz}$, this attenuation is offset by the increase in size of the mode shape at $91 \mathrm{~m}$, which causes both the source and the receiving hydrophones to couple to the first mode with increasing efficiency. The two effects almost cancel themselves, making the first mode received level appear relatively flat over this frequency range. Above $40 \mathrm{~Hz}$ the size of the first. mode shape at $91 \mathrm{~m}$ stops growing with frequency (as is seen in Figures 4.8 and 4-9). The balance between the two effects is lost, and the now severe first mode attenuation dominates the remaining results, causing the very quick drop in first mode received level that is seen in the data.

\subsection{Mode Coherence Measurements}

The results of the previous section indicate that the modal beamformer is performing reasonably well for frequencies of $30 \mathrm{~Hz}$ and below, where reliable array tilt estimates are available. This means that meaningful estimates of the coherence between the various modes can also be made. The results of these coherence measurements for the vertical array signals below $30 \mathrm{~Hz}$ are given in Table 7 -II. The window of data extracted from each signal to make the coherence estimate is the longest uninterrupted period available; the lone exception to this is the $23.5 \mathrm{~Hz}$ data, where the processing software limits the number of data points used. Air gun blasts, such as the one imbedded in the $17.75 \mathrm{~Hz}$ data, and other anomalies have been avoided so that the results would not be biased by invalid data.

The resultant coherence estimates are striking because of their uniformly large values. For all intents and purposes, these results show that the central Arctic channel can be considered to be a completely deterministic medium in time, and that all the different source-receiver propagation paths are completely phase locked. The slight variation that does exist between results appears to be strictly an issue of 


$\begin{array}{lccccc}\text { Frequency (Hz) } & \underline{15.00} & \underline{17.75} & \underline{20.00} & \underline{23.50} & \underline{30.00} \\ \text { Length of Average (sec) } & 400 & 2725 & 1120 & 2299 & 550 \\ \text { Mode 1-2 Pair } & 0.99 & 0.98 & 0.99 & 0.99 & 0.99 \\ \text { Mode 1-3 Pair } & 0.99 & 0.93 & 0.99 & 0.99 & 0.99 \\ \text { Mode 1-4 Pair } & - & - & - & 0.98 & 0.99 \\ \text { Mode 1-5 Pair } & - & - & - & - & 0.99 \\ \text { Mode 2-3 Pair } & 0.99 & 0.93 & 0.99 & 0.99 & 0.99 \\ \text { Mode 2-4 Pair } & - & - & - & 0.98 & 0.99 \\ \text { Mode 2-5 Pair } & - & - & - & - & 0.99 \\ \text { Mode 3-4 Pair } & - & - & - & 0.97 & 0.99 \\ \text { Mode 3-5 Pair } & - & - & - & - & 0.99 \\ \text { Mode 4-5 Pair } & - & - & - & - & 0.99\end{array}$

Table 7-II: Mode Coherence Estimates for the Vertical Array Data

SNR. The low levels of coherence in all pairings that include the third mode at 17.75 $\mathrm{Hz}$, for example, can be explained by realizing that the third mode amplitude is far enough below that of the first mode to allow the background noise level to exert a small amount of influence in the coherence estimate. Since confidence intervals for coherence estimates very close to unity are also quite tight [13], the estimates are guaranteed to be quite reliable from a statistical viewpoint.

While true coherence measurements cannot be made for vertical array signals above $30 \mathrm{~Hz}$, gross averages of observations over many different assumed array tilts are again possible. Like the lower frequency results, the modes at the higher frequencies also appear to be almost completely coherent. Modes that appear incoherent for a given tilt angle can normally be explained by an SNR argument. On the average, some small amount of coherence degradation can be seen at the higher frequencies, indicating that upper limits in both frequency and averaging length exist, beyond which the present results are not valid. Typical coherence values are about 0.96 for the $47 \mathrm{~Hz}$ data, and roughly 0.92 for the, $71 \mathrm{~Hz}$ data, 
compared to values of 0.99 seen at lower frequencies. None the less, these coherence levels are close enough to unity to conclude that the present experiment only covers a fraction of the frequency/duration space over which these conclusions are applicable.

hor scale: 200 sec / tick vert scale: $45^{\circ} /$ tick
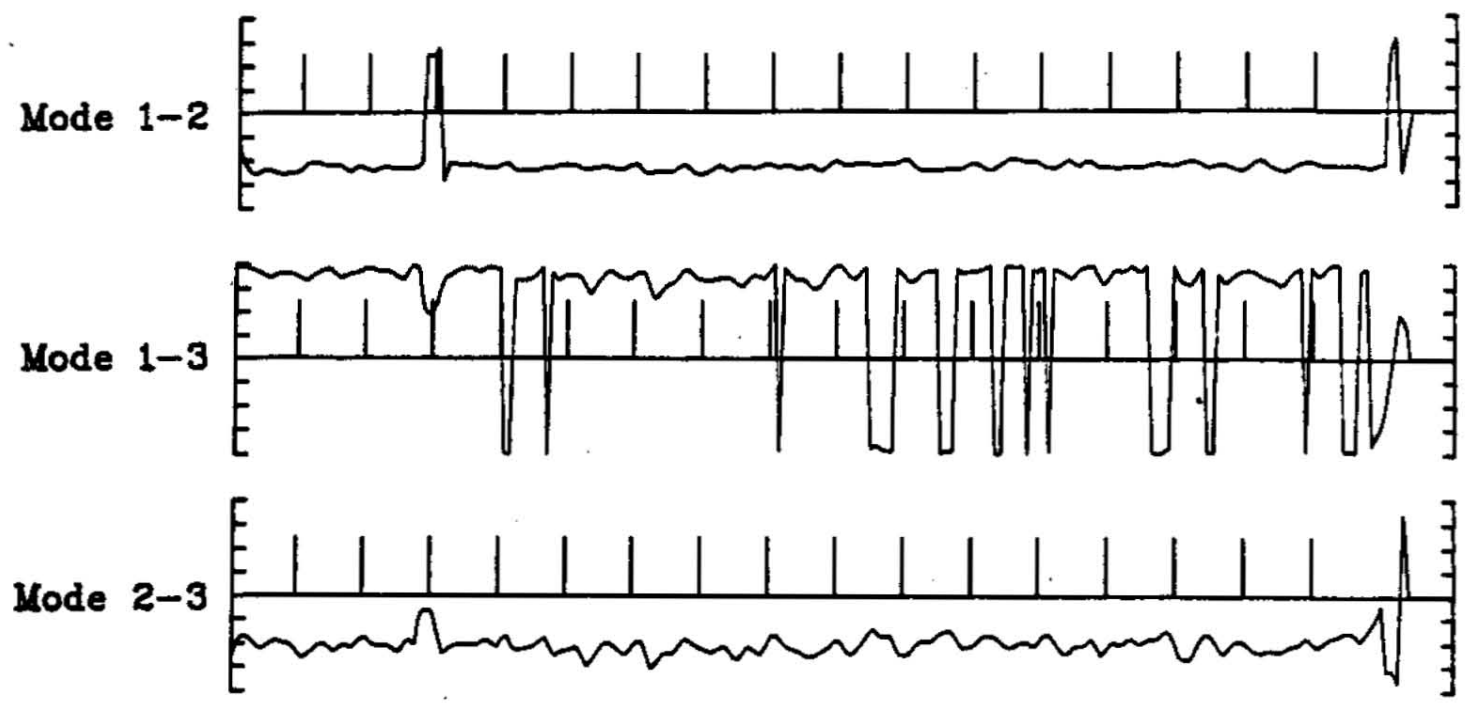

Figure 7-23: Time Series of the Relative Phases of Various Mode Pairs for $17.75 \mathrm{~Hz}$ Data

\subsection{Relative Phase Measurements for the Vertical Array Data}

The high levels of coherence just demonstrated between the various mode's indicate that their relative phases should be very stable over time. This is the case, as is seen in Figure 7-23. The three time series displaced represent the variations of the relative phases with time for the three possible pairings of the modes resolvable at $17.75 \mathrm{~Hz}$. As expected, the relative phases are seen to be extremely stable over the full 55 minute duration of the signal. The mode 1-2 pair is the most stable, 
demonstrating not more than a few degrees of fluctuation over the course of the signal. The somewhat larger fluctuations in the pairs involving the third mode reflect the same SNR issue discussed in the last section. Since the value of the mode 1.3 pair is very near to $180^{\circ}$, a large amount of phase wrap is visible.

Table 7-III gives the experimentally measured relative phases for all possible pairs of resolvable modes at frequencies below $30 \mathrm{~Hz}$. The signal durations used to compute these values are identical to those used in the coherence estimates. Assuming a source-receiver range of $254 \mathrm{~km}$ and a range independent channel, a comparison of the phases measured at $17.75 \mathrm{~Hz}$ with the values expected can be made. The results are presented in Table 7-IV. The significant differences between the theoretical and experimental data indicate two things. First, the calculation of the expected relative phase is sensitive to inaccuracies in the assumed horizontal phase speeds of the modal pairs. For example, a $1 \mathrm{~m} / \mathrm{sec}$ difference in the first mode phase speed results in about a $40^{\circ}$ in the predicted data of Table $7-\Gamma V$. Second, the measurement is also quite sensitive to range variations in the channel, since the total phase represents an integration of all the perturbations encountered in propagating through the channel. These appear to be non-negligible for this situation. Thus, while the relative phases themselves are quite stable and well behaved, there is some question as to whether it is possible to predict them accurately enough to accomplish passive source ranging in this fashion.

\subsection{Summary}

The horizontal and vertical array tonal data sets from the FRAM IV Arctic Acoustic Experiment that are described in Sections 3.9 and 3.10 have been processed using the algorithms discussed in Chapter 5. The results for both have been presented, compared, and analyzed in a variety of ways. 


$\begin{array}{lccccc}\text { Frequency (Hz) } & \underline{15.00} & \underline{17.75} & \underline{20.00} & \underline{23.50} & \underline{30.00} \\ \text { Mode 1-2 Pair } & 171^{\circ} & 102^{\circ} & 120^{\circ} & 48^{\circ} & 135^{\circ} \\ \text { Mode 1-3 Pair } & -153^{\circ} & -166^{\circ} & -22^{\circ} & -65^{\circ} & 174^{\circ} \\ \text { Mode 1-4 Pair } & - & - & - & -154^{\circ} & 85^{\circ} \\ \text { Mode 1-5 Pair } & - & - & - & - & 38^{\circ} \\ \text { Mode 2-3 Pair } & 36^{\circ} & 92^{\circ} & -142^{\circ} & -113^{\circ} & 39^{\circ} \\ \text { Mode 2-4 Pair } & - & - & - & 158^{\circ} & -50^{\circ} \\ \text { Mode 2-5 Pair } & - & - & - & - & -97^{\circ} \\ \text { Mode 3-4 Pair } & - & - & - & -89^{\circ} & -89^{\circ} \\ \text { Mode 3-5 Pair } & - & - & - & - & -136^{\circ} \\ \text { Mode 4-5 Pair } & - & - & - & - & -47^{\circ}\end{array}$

Table 7-III: Relative Phase Estimates for the Vertical Array Data

\begin{tabular}{lcc} 
Mode 1-2 Pair & Measured & Expected \\
\cline { 2 - 3 } & $102^{\circ}$ & $154^{\circ}$ \\
Mode 1-3 Pair & $-166^{\circ}$ & $-16^{\circ}$ \\
Mode 2-3 Pair & $92^{\circ}$ & $-170^{\circ}$
\end{tabular}

Table 7-TV: Comparison of Measured and Expected Relative Mode Phases for $17.75 \mathrm{~Hz}$ Data

The horizontal array data have been beamformed in azimuth using the single beam MLM algorithm in order to find the correct azimuth angles for the TRISTEN source. This processing results in azimuth angle estimates that are accurate to about $\pm 0.1^{\circ}$. The azimuth angles obtained agree completely with less precise estimates available from the navigation data. The MLM algorithm performs quite well in this application, producing a much narrower main lobe and significantly lower side lobe levels than an equivalent conventional beamformer.

The horizontal array has also been steered in the vertical direction at the azimuth angles previously determined to study the modal structure of the received field. This has been accomplished by varying the assumed horizontal phase speed with which the steering vector is computed. The processing has been accomplished 
twice: once with the single beam MLM algorithm to provide high resolution in phase speed; and once with the conventional beamformer to recover the correct received levels. The horizontal array data below $30 \mathrm{~Hz}$ exhibit a strong peak at horizontal phase speeds closely matching those predicted for the first mode. Above $40 \mathrm{~Hz}$, this first mode arrival vanishes; instead, a much more evenly distributed set of modal received levels is observed, possessing a very small peak at phase speeds corresponding to deep RSR path propagation. This occurs even though analysis of the modal structure suggests that near $40 \mathrm{~Hz}$, the received level of the first mode should dominate those of other modes by as much as $60 \mathrm{~dB}$. The region between 30 and $40 \mathrm{~Hz}$ might be considered to be a transition region between the two propagation regimes. Although a strong first mode arrival is seen in this band, it is perhaps not as great as might be expected from studies of the modal structure.

In the $27 \mathrm{~Hz}$ tone taken from the horizontal array, a strong fade similar to that seen by Mikhalevsky [57] has been identified. A study of the time series of this fade seems to indicate that it is a phase discontinuity which, in turn, causes the instantaneous frequency of the signal to walk out of the pass band of the low pass filter. Analysis in both the horizontal and the vertical shows no observable differences between the structure of the received field before and after the fade, suggesting that the cause is probably source related.

The vertical array data have been processed using the multiple beam least squares beamforming algorithm to produce modal amplitude and phase estimates. Three different types of output have been generated for the vertical array data set. One is a plot of the amplitude estimates for all the resolvable modes versus time for one given array tilt angle. The second is a plot of these same amplitude estimites, now displayed at one given time and across all reasonable values of array tilt. The third is a plot of the residual error versus time and assumed tilt angle. This last 
may be considered to be a condensation or integration into a single number of the all the various modal amplitude estimates made at one time and one assumed tilt angle. The three outputs represent different cross-sections along mutually orthogonal planes of an amplitude function that depends on mode number, time, and assumed tilt angle.

The modal decomposition of real data has been shown to be sensitive to the assumed array tilt in a fashion similar to the demonstrations made with synthetic data in Section 6.3. This sensitivity is seen to increase at higher frequencies and higher mode numbers. A tilt angle accuracy on the order of $\pm 0.25^{\circ}$ appears to be needed if one wishes to properly estimate all of the modes resolvable by the vertical array at any of the frequencies of interest. This requirement can be relased somewhat for lower frequencies and lower mode numbers.

As shown in Section 6.6, plots of residual error on the time-tilt plane allow estimation of the actual array tilt from the acoustic data. For the purposes of array tilt estimation, the residual error plots generated for the vertical array data have been used to compute one average tilt angle for the full duration of each signal, despite indications that the array may not be completely stationary over periods of longer than about 20 minutes. Where possible, the tilt angle estimates made by this procedure have been verified by comparing estimates from consecutively broadcast signals. For frequencies of $30 \mathrm{~Hz}$ and below, it appears that the estimation technique is capable of generating estimates to an accuracy of about $\pm 0.7^{\circ}$, with somewhat better results at the very lowest frequencies in the data set. This precision allows valid first mode estimates and partially valid second mode estimates to be made; at the lowest frequencies, third mode estimates may also be reasonably trustworthy. All tilt angle estimates made by this technique fall inside the expected limit of $5^{\circ}$. 
The residual error plots for signal frequencies above $30 \mathrm{~Hz}$ generally do not demonstrate the single deep minimum necessary to make accurate array tilt estimates. Even when estimates are possible, they do not appear to be consistent over consecutive signals. This problem is attributed to the increase at these frequencies of the proportion of energy carried by modes that are fundamentally unresolvable by the array. This effect has been demonstrated in the results of the horizontal array data. The outputs of vertical array modal decompositions versus both time and tilt angle also verify this effect. Above $30 \mathrm{~Hz}$, the first mode amplitude also appears to be abnormally low when compared to those of higher modes in the vertical array data.

The temporal stability of all the data examined in this chapter appears to be similar to that seen in the preprocessed time series of Section 3.12. In general, short term fluctuations for both horizontal and vertical array data are in the $1 \mathrm{~dB}$ range. Some larger short term variations have occasionally been noted on specific sensors in the horizontal array, but these do not appear to be coherent over the full array. Longer term variations (on the order of about 20 minutes) are seen in selected vertical array signals. The variations appear to increase as the frequency of the signal increases. No such long term fluctuations are observed in the horizontal array data. The lack of actual array tilt measurements makes the separation of channel fluctuation effects from array movement effects impossible, but nothing in the data precludes the latter from explaining the great majority of the long term variations seen.

The first mode amplitudes, as computed from the vertical array data, have been shown to be consistent with those derived from the horizontal array data over the range of frequencies (below $30 \mathrm{~Hz}$ ) where accurate estimates of each can be made. Both the horizontal and vertical array data indicate significant attenuation 
of first mode energy. Similar results are seen in the second and third mode amplitude results taken from the vertical array data. The nature of the attenuation curves versus frequency for the three modes demonstrates that the source of the attenuation is located in the Arctic surface duct. A good fit to the measured first mode attenuation can be generated by applying the method of small perturbations to a rough pressure release surface located at the ice-water interface, where the statistics used for the rough surface have been determined experimentally from ice profiles. A simpler approach using the Kirchhoff approximation yields only a very poor match to the measured attenuation values. Second and third mode attenuation estimates made with the method of small perturbations match experimental data somewhat less perfectly, predicting larger attenuation than is actually observed at higher frequencies. This result could possibly indicate that modal coupling between the first mode and neighboring modes cannot be ignored. If this is indeed the case, then the observed stability of the received signals requires the scattered field to be completely coherent with respect to the specular field, which, in turn, presumes a rough surface that is essentially stationary over time.

Coherence measurements for the various mode pairs have been made for signals with frequencies $30 \mathrm{~Hz}$ and below, where reliable tilt angle estimates are available. Average modal coherence over periods of as long as 45 minutes is typically 0.99 ; all the measurements made exceed a value of 0.93 . The few lower results observed appear to be caused by noise interference. By averaging results over a number of arbitrarily selected tilt angles, it is possible to get an indication of the coherence levels likely to be found at higher frequencies, where actual array tilt estimates cannot be made. Typical values are 0.96 at $47 \mathrm{~Hz}$ and 0.92 at $71 \mathrm{~Hz}$. Therefore, the various propagating modes for any signal in this data set can be considered to be completely coherent over the full duration of the transmission. 
The large coherence values observed also guarantee that they are highly reliable estimates. The slightly lower values seen at higher frequencies lead to the supposition that there is some frequency/duration limit beyond which these conclusions would no longer be valid.

The high levels of coherence observed guarantee that estimates of the relative phase of various mode pairs are meaningful. These relative phase estimates appear to be extremely stable, exhibiting only very small variations over time. However, the phases measured do not closely match those that would be expected if the channel were totally range independent. Thus, while the relative modal phases are quite stable, there appears to be some question about the ability to accurately predict them. 


\section{Chapter 8}

\section{Summary and Conclusions}

This chapter is divided into three sections. The first summarizes the general topics covered throughout the thesis, while the second discusses the major conclusions, locating them in the larger framework of signal processing theory and underwater acoustics propagation theory. A short list of some ideas for further work along these lines completes the thesis.

\subsection{Summary}

The twin issues of modal beamforming and mode coherence have been investigated at some length. The prime motivation for studying these topics comes from a desire to exploit the modal nature of sound propagation in a waveguide more fully, so that the source's range and depth may be estimated directly from sound field measurements. To this end, two necessary contributions have been made. First, beamforming techniques that address the waveguide nature of low frequency sound propagation in the oceans have been studied extensively and implemented on real data, including data taken from a vertical array; this has not been accomplished previously. Second, the relative amplitudes and phases of the various modes have been measured and their stability studied through the use of modal coherence estimates. This also is an original contribution of this thesis.

Some of the practical differences between horizontal and vertical arrays hive been analyzed. The most important difference from a signal processing viewpoint is the increased importance of vertical array sensor displacement. This is due to the increased size of the sensor displacements, which is caused by the greater scope of 
vertical arrays; and to the fact that the displacements occur in an organized pattern across the face of the array.

A preprocessing scheme involving two stages of quadrature demodulation, FIR low pass filtering, and decimation has been implemented to reduce the data set to a usable size prior to further processing. This scheme results a complex output time series for each channel that is demodulated to within $\pm 1 \mathrm{mHz}$ of the nominal signal frequency, filtered in a passband of $12.5 \mathrm{mHz}$, and downsampled to a sample interval of $1.6 \mathrm{sec}$. The resulting output time series contains about 100 independent degrees of freedom in a typical 55 minute signal.

The modal structure of the sound channel encountered during the FRAM $\mathrm{N}$. Experiment has been studied extensively, and predictions of the mode shapes and associated horizontal phase speeds have been made for all frequencies of interest here. The results have been analyzed both with respect to relative mode shape sizes at $91 \mathrm{~m}$ (the deployment depth for the TRISTEN source and the FRAM horizontal array) and with respect to horizontal phase speed variations as a function of frequency. The arrangement of the experiment makes both the source and the horizontal receiving array highly tuned to first mode propagation; first mode excitation typically exceeds that of other modes by more than $15 \mathrm{~dB}$. The variations of phase speed with frequency allow one to observe the transition of modes from the deeper sound speed profile into the strong Arctic surface duct. The effect of variations in the sound speed profile is shown to be small compared to the effect of the overhead ice canopy for central Arctic surface duct propagation.

Four different algorithms for computing modal decomposition of the observed sound field have been developed theoretically. These four algorithms include single beam and multiple beam variants of both the least squares and MLM algorithms. The least squares algorithm is shown to be a true least mean squares linear 
estimator for the complex modal amplitudes; its single beam variant is exactly the equivalent of the conventional beamformer. The MLM algorithm is shown to be a weighted least mean squares estimator, with the weighting essentially being the inverse of the sensor noise. The performance of all four methods has been compared.

In general, the multiple beam least squares technique can always be expected to provide better performance than its single beam counterpart, as long as the beams included together in the multiple beam approach are all different enough to avoid matrix singularity issues. Problems of this type are encountered when one begins to deal with modes not fundamentally resolvable to the array. The modal resolution of a vertical array may be limited in two ways, by its length and by inadequate inter-sensor spacing. An overly short array makes it difficult to resolve neighboring modes, while inadequate inter-sensor spacing causes Nyquist-like aliasing for modes widely separated in mode number. It has been demonstrated that the FRAM IV vertical array is array length limited for the low frequencies of interest in this thesis.

The single beam MLM algorithm can be expected to provide resolution superior to that of any of the other approaches in situations where the assumptions underlying the method are met. The most important of these assumptions recpuires that all the energy in the various modes be completely incoherent. The presence on coherent energy in several different beams causes severe distortions of the single beam MLM algorithm output, though. This coherent interference problem can be theoretically eliminated by the use of the multiple beam variant of the MLMI algorithm, but the practical realities of this approach make it unattractive. Thus. the multiple beam least squares processor has been chosen for use with the vertical array data analyzed here. It has been demonstrated that the single beam MLAI 
algorithm still provides higher resolution than either of the least squares approaches when properly used with horizontal arrays, even though the coherent interference problem corrupts the output levels. Therefore, the horizontal array data have been processed twice, once with the single beam MLM algorithm for resolution and once with the single beam least squares algorithm for the purpose of quantitative estimation of the received levels.

The effect of vertical array tilt on the modal decomposition process has been investigated. An examination of the fundamental physics of the process suggests that the modal beamformer results are very sensitive to variations in array tilt over the range of tilt angles most likely to be encountered with the FRAM IV vertical array, which is from $-5^{\circ}$ to $5^{\circ}$ away from vertical. This examination also leads to a simple method for estimating such tilt angle sensitivity. Upon application to the FRAM IV vertical array, it is found that the tilt angle must be known to about $\pm 0.25^{\circ}$ in order to properly resolve anything more than the first mode. This sensitivity to tilt angle increases at higher mode numbers and higher frequencies. Since no tilt angle measurements are available for the FRAM IV Experiment, a scheme for estimating the array tilt from the acoustic data has been developed and implemented in this thesis. The method involves the minimization of the residual error associated with the multiple beam least squares beamforming algorithm over a reasonable range of assumed array tilt angles. This scheme has been shown capable of tracking the array in tilt angle by simulation; its performance is dependent upon the total amount of energy present in the modes that are included in the beamformer relative to the amount present in modes not resolvable by the array. The approach is subject to bias when a large percentage of the signal energy that is present resides in fundamentally unresolvable modes.

Two extensive tonal data sets taken during the FRAM IV Arctic Acontic 
Experiment have been processed and analyzed in this thesis. One set was recorded from the hydrophones of the horizontal array available at the FRAM ice camp, while the other set was recorded from eighteen sensors of the vertical array deployed at the same camp. The horizontal array data have been analyzed to provide experimental amplitude and horizontal phase speed measurements for the first mode; these, in turn, have been used to successfully test the consistency of the modal beamformer output and the validity of the mode structure determined in Chapter 4. The vertical array data has been used to measure modal amplitudes and relative phases for various low order modes, and to make estimates of the temporal stability and coherence of the complex modal amplitudes over time. The modal amplitudes measured have been analyzed with respect to the attenuation observed. and measured modal attenuation levels have been compared with predicted levels using both the Kirchhoff approximation and the full method of small perturbations. Good agreement with experimental attenuation results has been obtained from the latter method using a simple rough free surface model and experimentally derived estimates of the ice canopy statistics. The stability and coherence measurements have been used to investigate the nature of the phase relationship between various modes. It has been concluded that the central Arctic sound channel can be considered to be completely deterministic in terms of signal propagation over periods of time well in excess of one hour. All propagating modes appear to be phase-locked, indicating that no independent, phase-random propagation paths exist in the Arctic for these time scales and operating frequencies. 


\subsection{Conclusions}

The important conclusions of this thesis fall into the same three general categories into which the contributions discussed in Section 1.4: results pertaining primarily to the discipline of signal processing; results dealing with the practical use of vertical arrays; and results affecting the field of underwater acoustics. Each category is discussed in turn.

\subsubsection{Signal Processing Conclusions}

The most important signal processing conclusion is that a method for decomposing the sound field generated by a distant tonal source and received at a vertical array into its component normal modes has been proven to be effective by theory, by simulation, and by practical application to real data. This last is particularly significant, as no previous work dealing with narrowband modal beamforming has involved application to actual data taken in the field. Thus, this thesis demonstrates the feasibility of estimating narrowband modal amplitudes and phases directly from data taken with a vertical array.

A number of conclusions have been reached concerning the relative performance of the four beamforming algorithms investigated, primarily along the lines of comparisons between the least squares and MLM approaches and between single beam and multiple beam algorithms.

The critical issue involved in any comparison of MLM and least squares beamforming algorithms for narrowband modal applications is the coherence of the various modal amplitudes. What is defined as the signal for the MLAI algorithm must be incoherent with respect to everything else in the sound field. which is automatically defined as noise. Violation of this requirement has been shown to have severe consequences for MLM performance, particularly when the steering 
vectors that represent the various beams of interest approach orthogonality; such is the case for the low order modes that are of interest here. If it is desired to use the MLM algorithm when highly coherent signals are simultaneously present on different beams, then a multiple beam approach that includes all possible beams upon which the coherent energy can arrive must be used. Alternatively, it may be possible to create an effective steering vector from a properly weighted sum of the beams. The former approach is generally infeasible, because the number of beams that must be included almost always exceeds the number of sensors available, so that the MLM inversion becomes singular. In the rare cases when an adequate number of sensors is available, one can still expect to encounter problems with the processing stability of the algorithm. On the other hand, the latter approach generally requires one either to index the beamforming over an unacceptably liarge number of parameters (all possible relative modal amplitudes and phases), or to somehow solve the modal propagation problem a priori, such as through the use of normal mode or parabolic equation predictions. Although it is clearly not of use for the present effort, this final method does offer some promise.

It is interesting to note that in situations where the MLM algorithm has been traditionally employed, namely, for horizontal arrays when high resolution is needed, the effects of the coherent interference problem are minimized. Higher resolution than that of conventional approaches is in fact achieved, at the expense of accuracy in the absolute levels computed. This effect has probably been incorrectly attributed to the issue of MLM bias in the past, masking the coherent interference problem.

By comparison, the performance of the least squares algorithms is not sensitive to the issue of coherence between signal and noise, making it much more robust. Additionally, because the technique is linear, it does not suffer from the bias issues 
known to plague MLM algorithms. Still, the results of Chapter 5 indicate that least squares approaches provide significantly less resolution than MLM techniques when the latter can be applied.

The major difference between multiple beam algorithms and their single beam equivalents stepped over the same set of steering vectors is that the multiple beam processors force the decoupling of the various beams while the single beam variants do not. Stated another way, each beam included in a multiple beam processor has its nulls arranged in such a fashion to prohibit the leakage of energy detected on the other beams included in the processor. Whether or not this null placement improves performance over that of the equivalent single beam processor depends on two things. First, the way in which the degree of freedom that each specially placed null represents is employed in the single beam approach must be considered. For the least squares algorithm, the single beam processor does not use null placement to improve the results; thus, the multiple beam least squares processor can be expected to provide at least marginally better performance by eliminating the crosstalk between beams. In the MLM algorithm, however, the single beam processor deploys the nulls in such a fashion as to minimize the total noise power allowed into the beam. Thus, the multiple beam MLM processor decouples the included beams only at the expense of less satisfactory noise rejection performance. If the energy in the different beams is incoherent, this is always a losing proposition in terms of total performance, as is shown in Appendix B. On the other hand, if the energy is coherent across different beams, then the single beam MLM processor experiencess serious difficulties due to the coherent interference, and the multiple beam ML.M approach must be used.

The other thing that must be considered when discussing single beam versus multiple beam approaches is the processing stability issue. If the different beams 
that one is trying to decouple with the multiple beam algorithm look too much alike, then the decoupling process becomes singular; the beamformer must work so hard to separate the closely spaced beams that it becomes sensitive to even small amounts of background noise. The conclusion made here is that the issue can be quantified for the least squares algorithm through the use of the singularity coefficient defined in Section 5.10. Significant processing stability problems begin to occur at singularity coefficient values of roughly 0.4 . For modal beamforming with vertical arrays, the problem arises from the fundamental inability of the array to resolve higher order modes. Two causes are possible: insufficient array length, which causes adjacent modes to look too much alike; and insufficient inter-sensor spacing. which causes Nyquist-like aliasing between modes widely spaced in mode number. The FRAM IV vertical array has been shown to be array length limited for the low frequencies of interest here.

The final conclusion of this section deals with the usefulness of the residual error as a performance measure for the least squares algorithm. Since the residual error essentially provides a goodness of fit measurement, it can, if used carefully, be successfully employed to estimate critical unknown parameters from the acoustic data. One example is its use in finding the array tilt angle. Its reliability in accomplishing such a task is fundamentally related to the percentage of signal energy in the modes resolvable to the vertical array. When a large proportion of the signal energy resides in fundamentally unresolvable modes, then the method can be expected to work poorly, since the implicit signal model is inadequate. This effect has been demonstrated with real data, where reliable estimates of array tilt for signals over $40 \mathrm{~Hz}$ cannot be made. 


\subsubsection{Conclusions Concerning the Practical Use of Vertical Arrays}

Three major conclusions deal with the practical employment of vertical arrays in a real ocean environment. The first, which has already been mentioned, is the modal resolution issue. The techniques developed in Chapter 5 are more than adequate for investigating this question for arbitrary vertical arrays, and can even be inverted for use in a design role, such as selecting sensor depths to maximize the singularity coefficient.

From the comparisons made in Sections 3.5, 3.6, and 3.7, it can be concluded that the proper design and implementation of vertical arrays is a much harder process than the equivalent development of horizontal arrays. Variations in hydrophone sensitivity across the array must be very carefully controlled, since typical applications of vertical arrays depend more heavily on accurate relative signal levels between different sensors than do those of horizontal arrays. The greater scopes of vertical arrays significantly increase the effect of ocean currents on array shape and, therefore, on sensor location, to the point where the net sensor displacements can no longer be neglected in the signal processing. This increased importance is due both to larger physical sensor offsets and to the fact that these offsets now occur in an organized pattern across the face of the array.

The results of Chapter 6 and Chapter 7 leave little doubt about the conclusion that modal beamforming techniques are extremely sensitive to variations in array tilt. This sensitivity increases for higher order modes and with higher operating frequency. The combination of this conclusion with the previous one shows that improved methods of either controlling or measuring vertical array shape, and particularly the effective tilt angle of the array, are needed for proper exploitation of the unique capabilities of vertical arrays. From Section 6.4, it can be concluded that the effective array tilt must be known to better than about $\pm 0.25^{\circ}$ in the 
Arctic Ocean if one wishes to accurately measure the amplitudes and phases of just the lowest order modes in the 0 to $80 \mathrm{~Hz}$ frequency range. A simple method of predicting the array tilt sensitivity has been presented there. It is important to note that the results of Chapter 3 and Chapter 7 indicate the shortest time scales for significant tilt angle variations in the FRAM IV vertical array are between 10 and 20 minutes.

\subsubsection{Underwater Acoustics Conclusions}

The results of Chapter 7 paint a fresh and unique picture of low frequency sound propagation in the central Arctic, from which a number of important conclusions can be drawn.

The validity of all the other results obtained in this area is dependent upon the fundamental conclusion that the modal structure developed in Chapter 4 is a reasonably accurate representation of that present during the FR.AII IV Experiment. This inference can be readily drawn from Figure 7-7, since, in general. modal phase speeds tend to be much more sensitive to channel variations than mode shapes.

The next significant conclusion to be drawn concerns the importance of the Arctic surface duct in sound propagation, even at the lowest frequencies. This duct influences the FRAM IV sound propagation problem in three ways: by controlling the modal distribution of source energy; by controlling the sensitivity of the horizontal array to the various modes; and by concentrating large amounts of sound energy very near the rough ice canopy. It is obvious that the overwhelming majority of sound energy generated by any source located in the surface duct is channeled into modes that are effectively trapped in that duct. A similar conclusion is true for horizontal receiving arrays deployed within the surface duct. For the 
frequencies of interest here, this means that the first mode has a tremendous relative advantage over all other modes; first mode source excitation is often more than $15 \mathrm{~dB}$ above that for other modes. It is also interesting and important to note the frequencies at which various modes transition into the surface duct. The first mode crosses over from the deeper Arctic profile to the surface duct at about $20 \mathrm{~Hz}$. the second mode at about $40 \mathrm{~Hz}$, and the third at roughly $65 \mathrm{~Hz}$, although they are not completely trapped in the upper layer until frequencies of about $30 \mathrm{~Hz}, 60 \mathrm{~Hz}$. and $90 \mathrm{~Hz}$, respectively.

Given the great relative advantage that the first mode possesses compared to the higher order modes, the observation of only very small amounts of first mode energy at frequencies above $40 \mathrm{~Hz}$ is extremely important. It highlights the importance of modal attenuation effects in central Arctic Ocean propagation. In terms of what is observed from horizontal array data, two very different propagation regimes exist. One of these regimes occurs below $40 \mathrm{~Hz}$, where strong first mode arrivals are observed on the horizontal array. The other regime, above $40 \mathrm{~Hz}$, shows a much more equal partition of energy in the higher order modes, with the deepest RSR paths being marginally dominant. Comparison with vertical array results explains this sharp change. At frequencies below $40 \mathrm{~Hz}$, the increase in attenuation of the first mode with frequency is approximately compensated by increasing source excitation and horizontal array sensitivity. Only above $40 \mathrm{~Hz}$, when these effects no longer mask it, does the strong frequency dependence of the first mode attenuation become apparent in the horizontal array data. The $40 \mathrm{~Hz}$ breakpoint between the two regimes can be considered to be an effective upper bound to the range of frequencies that propagate efficiently in the Arctic surface duct, and may thus be taken as a high frequency limit for long range acoustic systems designed to be employed in the Arctic environment. 
The modal attenuation measurements made for the first three modes from the vertical array data verify the dominant role that the rough surface of the ice canopy plays in Arctic Ocean sound attenuation. The frequency dependence of the attenuation for the different modes correlates well with the frequencies at which they transition into the surface duct, suggesting that it is the ice cover that provides the attenuation mechanism. Moreover, the agreement of the measured modal attenuation with predictions using the full method of small perturbations and concurrent experimental estimates of the ice statistics is excellent for a model consisting solely of a rough free surface at the ice-water interface. This result is at variance with that of DiNapoli and Mellen [25], in which they cannot reach adequate agreement between experiment and theory using measured values of the ice statistics. The reason for the difference in results is not presently understood. The Kirchhoff approximation is shown to be poor by comparison in this situation. The result demonstrates the need for the careful inclusion of surface correlation effects in rough surface scattering models. Additionally, it is possible to interpret the second mode attenuation results as indicating that multiple scattering effects, or, equivalently, modal coupling effects, are significant in the rough surface scattering problem posed by the ice canopy. Certainly, a great deal more work is needed to verify this interpretation; if it is correct, however, it demonstrates a unique case where the modal coupling is significant even though the coupling coefficients themselves are negligible. What would make the coupling important in this case is the great difference in modal excitation levels. Even very low level scattering from the first mode into the second or third modes may be important when the original excitation levels of these modes are 20 or $30 \mathrm{~dB}$ smaller than that of the first mode.

The signal stability observed throughout this investigation has been 
remarkable. Many of the temporal variations observed can be attributed either to background noise effects or to vertical array movement, although assignment of observed fluctuations to the latter cause cannot be done conclusively. This is due to the lack of concurrent vertical array tilt measurements for the experiment. The only exceptions to these findings are some signal fades observed only on selected hydrophones of the arrays used; the cause of these variations is not presently well understood. The observed stability of the central Arctic sound channel and the high values of the modal coherence measured strongly support the conclusion that, for the low frequencies investigated here, the central Arctic channel can be considered to be completely deterministic in a temporal sense for periods in excess of one hour. These results agree closely with those of Mikhalevsky [5i], but also break new ground, since the various propagation paths have now been at least partially separated for independent inspection. Not only are the relative phases of the various modes very stable; it appears that the environment causes almost no phase fluctuations of any kind. Thus, the different propagation paths appear to be completely coherent, not so much because they all exhibit the same phise fluctuations, but more because none of them exhibit any significant phase fluctuations at all. This observation has a number of important implications. First, it means that any energy scattered due to rough surface effects (particularly from the ice) remains coherent with respect to the transmitted field. This is why sound transmissions above $40 \mathrm{~Hz}$, while obviously suffering major amounts of scattering by the ice, still display such stable phase traces at the different sensors. Second, it means that totally unrelated tonal sources can still exhibit very high cross-coherence in the central Arctic; the primary limiting factor is the ability to match the different sources in frequency. This, in turn, makes the use of high resolution beamforming algorithms that are subject to coherent interference (such as the MLM algorithm) 
highly suspect for stationary (non-moving) tonal sources in the central Arctic, unless either only a single source is known to be present, or the averaging length is extended to periods of at least several hours, if not several days. Most importantly, the observations show that the channel does not destroy the relative phase information from which target range information might be extracted. Therefore. target range estimation is feasible with respect to the temporal fluctuations in the central Arctic. What is not so clear from the results of this investigation is whether relative phase predictions can be made accurately enough to support such a passive ranging effort, especially if multiple scattering or mode coupling effects are, in fact. significant.

In retrospect, it is interesting to apply the insight gained from the Arctic data presented here back to the open oceans of the world. Clearly, these warmer waters do not exhibit anything near the same stability and coherence as observed in the central Arctic. Since the most glaring difference between the two environments is the type of free surface involved, one must at least suspect that the temporal instabilities more regularly observed in oceanic sound transmission are intimately related to the time variations in this free surface from wind and wave action. Certainly, these effects generate the strongest temporal variations in channel. The Arctic results prove that temporal channel variations are needed to generate temporal fluctuations in the propagating sound field.

\subsection{Some Thoughts on Further Work}

Every scientific investigation answers certain questions while raising other. previously unseen, issues. This thesis is certainly no exception. Before concluding, then, it is useful to discuss at least a few areas that may prove fruitful for further research. 
From a signal processing viewpoint, two valuable investigations come to mind immediately. The first is the issue of coherent interference in the MLAI algorithm. For a situation such as that found in the central Arctic Ocean, no high resolution beamforming alternative that presently exists could be expected to work well, because of the coherent nature of the channel. An investigation of how one might successfully employ high resolution concepts in this type of environment would be of great interest, not only for its practical value, but also because it would probably provide a great deal of insight into how present high resolution algorithms might be made more reliable. It is this lack of robustness which often inhibits potential users from employing high resolution techniques. Additionally, such an investigation would probably remove much of the mystery surrounding the often misunderstood MLM bias issue.

A second useful investigation would deal with better methods of inferring vertical array tilt from a received acoustic field. If a highly accurate method of accomplishing this task can be found (one much more reliable than the technique used here), it would provide a rather cheap and simply implemented solution to the most important problem facing anyone wishing to use vertical arrays for modal decomposition. By comparison, any other type of solution, such as an in situ measurement scheme or a modified deployment method, is likely to be both cumbersome and expensive. A related effort to this would be an investigation of the effects of higher order array shape variations on modal beamforming. Although assumed negligible here, there is likely to be some frequency/mode number limit beyond which even the small amounts likely to be encountered in practice are significant. Such an investigation is fairly straightforward, and would add confidence to the belief that the shape of a vertical array can be adequately modeled by a straight line at some angle to the vertical. 
The most interesting effort that could be undertaken in the underwater acoustics domain to follow the present work would be a repeat of this study in an open ocean situation. Lacking that, two other related investigations come to mind. First, the indications that mode coupling might be significant for the rough surface scattering observed need to be studied more closely. Intimately coupled to that question is the more difficult issue of whether or not relative modal phases can be predicted from the general characteristics of the medium with enough accuracy to allow the source range to be estimated. Such an investigation would obviously have to include an assessment of the effects of mode coupling.

Finally, it would be both useful and enjoyable to repeat the exact same experiment with an adequate array tilt measurement system in place. The lack of tilt angle measurements has caused more than a little frustration in this thesis, and in too many ways the results are still tenative as a result. In particular, it would be interesting to know just how effective the array tilt estimation scheme used here is in practice, since this is probably the greatest remaining unknown affecting the results presented here. 


\section{Appendix A \\ On the Inclusion of Signal \\ Components in Generalized Least Squares Weighting Matrices}

Consider a generalized least squares estimation problem with an $N \times N$ Hermitian positive definite weighting matrix $\underline{\mathbf{W}}_{0}$, and an $N \times M$ complex steering matrix $\underline{\mathbf{E}}$. The $M \times 1$ complex vector $\underline{\mathbf{A}}_{0}$ which minimizes the scalar error $Q$ defined in equation (5.14) is given in equation (5.15), which is repeated for convenience

$$
\underline{\hat{\mathbf{A}}}_{0}=\left(\underline{\overline{\mathbf{E}}}^{+} \underline{\overline{\mathbf{W}}}_{0} \underline{\overline{\mathbf{E}}}\right)^{-1} \underline{\underline{\mathbf{E}}}^{+} \underline{\mathbf{W}}_{0} \underline{\mathbf{P}} .
$$

Here, $\underline{\mathbf{P}}$ is the observed complex pressure vector.

Typically, the desired weighting matrix is the inverse of the sensor crosscoherence matrix for the noise. However, it is usually more convenient to use the total sensor cross-coherence matrix, including both the signal and noise components. since estimation of this matrix does not require separating the signal from the noise. Thus, one must be concerned with the effect of including signal components in the inverse of the weighting matrix. Let $\underline{\overline{\mathbf{W}}}$ be such a modified weighting matrix. Its form can be defined by

$$
\underline{\mathbf{W}}^{-1}=\overline{\mathbf{W}}_{0}^{-1}+\underline{\overline{\mathbf{E}}} \underline{\mathbf{B}} \underline{\mathbf{B}}^{+} \underline{\overline{\mathbf{E}}}^{+},
$$

where the $M \times 1$ complex vector $\underline{B}$ consists of arbitrary constants. The second term of equation (A.2) represents the inclusion of the signal components. Using the identity 


$$
\left(\underline{\overline{\mathbf{Z}}}+\underline{\mathrm{U}} \underline{\mathbf{V}}^{+}\right)^{-1}=\underline{\overline{\mathrm{Z}}}^{-1}-\frac{\overline{\mathrm{Z}}^{-1} \underline{\mathrm{U}} \underline{\mathbf{V}}^{+} \overline{\mathbf{Z}}^{-1}}{1+\underline{\mathbf{V}}^{+} \underline{\overline{\mathbf{Z}}}^{-1} \underline{\mathrm{U}}},
$$

it is possible to generate an expression for $\underline{\overline{\mathbf{W}}}$ in closed form

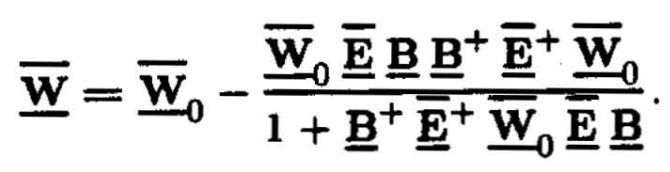

The optimal estimator for $\overline{\overline{\mathbf{W}}}$ is

$$
\underline{\hat{\mathbf{A}}}=\left(\underline{\overline{\mathbf{E}}}^{+} \underline{\overline{\mathbf{W}}} \underline{\overline{\mathbf{E}}}\right)^{-1} \underline{\bar{E}}^{+} \underline{\overline{\mathbf{W}}} \underline{\mathbf{P}} \text {. }
$$

Using equation (A.4), it is possible to write

$$
\underline{\overline{\mathbf{E}}}^{+} \underline{\overline{\mathbf{W}}} \underline{\overline{\mathbf{E}}}=\left(\underline{\overline{\mathbf{E}}}^{+} \underline{\mathbf{\mathbf { W }}}_{0} \overline{\overline{\mathbf{E}}}\right)-\frac{\left(\overline{\overline{\mathbf{E}}}^{+} \overline{\overline{\mathbf{W}}}_{0} \overline{\overline{\mathbf{E}}}\right) \underline{\mathbf{B}}^{+} \underline{\underline{\mathbf{B}}}^{+}\left(\overline{\overline{\mathbf{E}}}^{+} \overline{\overline{\mathbf{W}}}_{0} \overline{\overline{\mathbf{E}}}\right)}{1+\underline{\mathbf{B}}^{+} \overline{\mathbf{E}}^{+} \underline{\overline{\mathbf{W}}}_{0} \overline{\overline{\mathbf{E}}} \underline{\mathbf{B}}} .
$$

which can again be inverted using equation (A.3) to yield

$$
\left(\underline{\bar{E}}^{+} \overline{\overline{\mathbf{W}}} \underline{\bar{E}}\right)^{-1}=\left(\overline{\bar{E}}^{+} \underline{\overline{\mathbf{W}}}_{0} \overline{\overline{\mathbf{E}}}\right)^{-1}+\underline{\mathbf{B}}^{+} \text {. }
$$

Substituting equations (A.4) and (A.7) into equation (A.5) yields

$$
\underline{\hat{\mathbf{A}}}=\left(\underline{\overline{\mathbf{E}}}^{+} \underline{\overline{\mathbf{W}}}_{0} \underline{\mathbf{E}}^{-1} \underline{\underline{\mathbf{E}}}^{+} \underline{\overline{\mathbf{W}}}_{0} \underline{\mathbf{P}}=\underline{\hat{\mathbf{A}}}_{0}\right. \text {. }
$$

It can be seen that the least squares estimator produces the same result for either of the two weighting matrices. If $\underline{\underline{\mathbf{W}}}_{0}$ is taken to be the inverse of the noise portion of the sensor coherence matrix, then, from equation (A.2), $\underline{\overline{\mathbf{W}}}$ is the inverse of the full sensor coherence matrix (including both signal and noise) for a completely coherent signal that is uncorrelated with the noise. Note that this is the nature of the requirement for independent signal and noise in the MLM algorithm.

The above result can easily be generalized to the case of a random signal that 
is less than fully coherent. In this situation, the relation between the two weighting matrices is of the form

$$
\underline{\overline{\mathbf{W}}}^{-1}=\overline{\mathbf{W}}_{0}^{-1}+\underline{\overline{\mathbf{E}}} \overline{\mathbf{S}}_{M} \underline{\overline{\mathbf{E}}}^{+} \text {, }
$$

where $\overline{\mathbf{S}}_{M}$ is the $M \times M$ modal (or signal) cross-coherence matrix

$$
\overline{\mathbf{s}}_{M}=\mathrm{E}\left[\underline{\mathbf{B}} \underline{\mathbf{B}}^{+}\right] \text {. }
$$

This is a complete generalization, since for complete signal coherence the vector $\underline{\mathbf{B}}$ becomes non-random, and the result then reverts back to that given in equation (A.2).

By singular value decomposition, the matrix $\overline{\mathbf{S}}_{M}$ can be written as

$$
\overline{\underline{\mathbf{s}}}_{\mathrm{M}}=\sum_{i=1}^{M} \lambda_{i} \underline{\mathbf{e}}_{i} \mathbf{e}_{i}^{+}
$$

where the $\lambda_{i}$ and $\underline{e}_{i}$ are its eigenvalues and eigenvectors, respectively. The more general result is then proven by applying the approach presented above $M$ times in succession, once for each eigenvector in the sum. It is important to observe that while this generalization relaxes the deterministic signal assumption. it in no way alters the requirement that the signal and noise be completely uncorrelated. 


\section{Appendix B \\ The Performance Relationship Between Single Beam and Multiple Beam MLM Algorithms}

Consider two related multiple beam MLM estimation problems. In the first problem, $M$ modes (or beams) are included in the beamformer. From equation (5.20), the estimate of the $M \times M$ modal (or beam) cross-coherence matrix for this situation is

$$
\hat{\mathbf{S}}_{M}=\left(\overline{\mathbf{E}}_{M}^{+} \overline{\mathbf{S}}_{\mathrm{N}}^{-1} \overline{\mathbf{E}}_{M}\right)^{-1}
$$

where $\overline{\mathbf{E}}_{\mathrm{M}}$ is the $N \times M$ steering matrix and $\overline{\mathbf{S}}_{\mathrm{N}}$ is a sensor cross-coherence matrix of size $N \times N$.

As the second problem, consider a multiple beam beamformer having a total of $M+1$ modes included, and let the first $M$ of these modes be identical to those from above. The steering matrix for this problem is then $N \times M+1$, and may be written as

$$
\overline{\mathbf{E}}_{\mathrm{M}+1}=\left[\overline{\underline{\mathbf{E}}}_{\mathrm{M}} \mid \underline{\mathbf{E}}\right]
$$

where $\mathbf{E}$ is the $N \times \mathbf{l}$ steering vector of the additional mode. The estimate of the $M+1 \times M+1$ modal cross-coherence matrix is

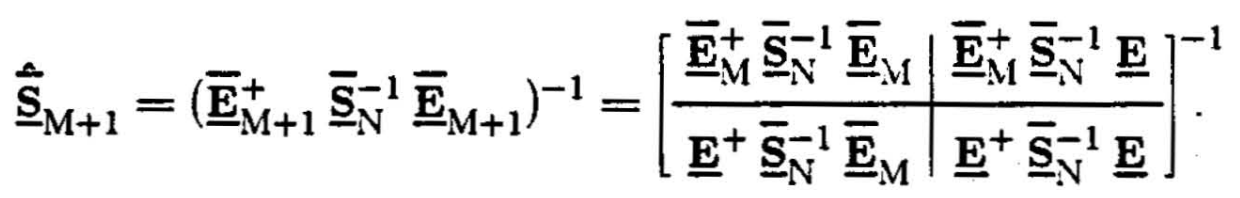

An estimate of the modal cross-coherence matrix for the original $M$ modes may be obtained from the second problem by extracting the upper left hand $M \times M$ submatrix 


$$
\underline{\hat{\mathbf{S}}}_{\mathrm{M}+1}=\left[\begin{array}{c|c}
\underline{\hat{\mathbf{S}}}_{\mathrm{M}}^{\prime} & \underline{\hat{\mathbf{R}}} \\
\hline \underline{\hat{\mathbf{R}}}^{+} & \hat{\sigma}_{M+1}^{2}
\end{array}\right] .
$$

$\underline{\hat{\mathbf{S}}}_{M}^{\prime}$ may then be identified by using the formula for the inverse of a partitioned Hermetian matrix. If

$$
\underline{\overline{\mathbf{x}}}=\left[\begin{array}{l}
\underline{\overline{\mathbf{Y}}} \mid \underline{\mathbf{Z}} \\
\hline \underline{\mathbf{z}}^{+} \\
v^{2}
\end{array}\right],
$$

and

$$
\underline{\overline{\mathbf{x}}}^{-1}=\left[\begin{array}{l|l}
\underline{\overline{\mathbf{A}}} & \underline{\mathbf{B}} \\
\hline \underline{\mathbf{B}}^{+} & c^{2}
\end{array}\right],
$$

then

$$
\begin{aligned}
& \underline{\overline{\mathbf{A}}}=\underline{\overline{\mathbf{Y}}}^{-1}+\frac{\overline{\overline{\mathbf{Y}}}^{-1} \underline{\mathbf{Z}} \underline{\mathbf{Z}}^{+} \underline{\overline{\mathbf{Y}}}^{-1}}{v^{2}-\underline{\mathbf{Z}}^{+} \underline{\mathbf{Y}}^{-1} \underline{\mathbf{Z}}}, \\
& \underline{\mathbf{B}}=\frac{-\underline{\overline{\mathbf{Y}}}^{-1} \underline{\mathbf{Z}}}{v^{2}-\underline{\mathbf{Z}}^{+} \underline{\underline{\mathbf{Y}}}^{-1} \underline{\mathbf{Z}}},
\end{aligned}
$$

and

$$
c^{2}=\frac{1}{v^{2}-\underline{\mathbf{Z}}^{+} \underline{\overline{\mathbf{Y}}}^{-1} \underline{\mathbf{Z}}}
$$

From equation (B.7),

$$
\begin{aligned}
& \underline{\hat{\mathbf{s}}}_{\mathrm{M}}^{\prime}=\left(\underline{\overline{\mathbf{E}}}_{\mathrm{M}}^{+} \overline{\overline{\mathbf{s}}}_{\mathrm{N}}^{-1} \overline{\mathbf{E}}_{\mathrm{M}}\right)^{-1}+
\end{aligned}
$$

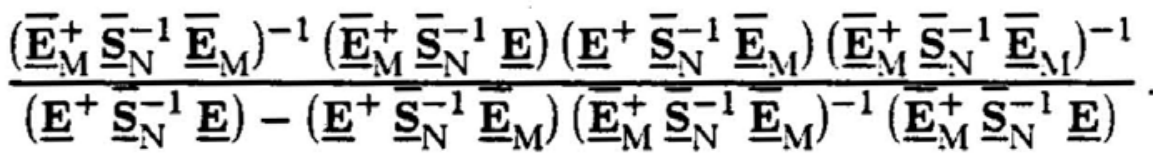


Equations (B.1) and (B.10) can be interpreted to show that the beamformer containing $M$ modes always provides better performance than the one containing $M+1$ modes. Consider a situation where the sensor cross-coherence matrix consists solely of noise with no signal. For these conditions, the two different estimates of the $M \times M$ modal cross-coherence matrix indicate the amount of noise that is not rejected in each beamformer. In particular, the diagonal terms indicate the noise power that leaks into the amplitude estimate of each mode. A comparison of equation (B.1) with (B.10) shows that the diagonal terms in the latter are never less than the diagonal terms in the former. The additional contributions indicated in (B.10) are always positive, since the numerator of the fraction is a matrix formed by taking an outer product of a vector with itself, while the denominator is a scalar that is also guaranteed to be positive. This last conclusion comes from a consideration of $\hat{\sigma}_{M+1}^{2}$ in equation (B.4). If one assumes $\overline{\mathbf{S}}_{\mathrm{N}}$ to be positive definite, then it follows that $\stackrel{\widehat{S}}{M+1}_{M}$ must also be positive definite and, hence, $\hat{\sigma}_{M+1}^{2}$ must be positive. But, from equation (B.9),

$$
\begin{aligned}
& \dot{\sigma}_{M+1}^{2}= \\
& \frac{1}{\left(\underline{\mathbf{E}}^{+} \underline{\overline{\mathbf{S}}}_{\mathrm{N}}^{-1} \underline{\mathbf{E}}\right)-\left(\underline{\mathbf{E}}^{+} \underline{\underline{\mathbf{S}}}_{\mathrm{N}}^{-1} \underline{\underline{\mathbf{E}}}_{\mathrm{M}}\right)\left(\overline{\underline{\mathbf{E}}}_{\mathrm{M}}^{+} \overline{\overline{\mathbf{S}}}_{\mathrm{N}}^{-1} \overline{\underline{\mathbf{E}}}_{\mathrm{M}}\right)^{-1}\left(\underline{\mathbf{E}}_{\mathrm{M}}^{+} \overline{\underline{\mathbf{S}}}_{\mathrm{N}}^{-1} \underline{\mathbf{E}}\right)},
\end{aligned}
$$

so that the denominator (which is identical to that in equation (B.10)) must be positive.

More insight can be gained by considering a situation where the sensor crosscoherence matrix is a combination of two components, one consisting of noise and the other being a signal of amplitude $\alpha$ in the $M+1^{\text {st }}$ mode. From the viewpoint of the first $M$ modes, the signal in the $M+1^{\text {st }}$ mode represents additional noise against. which the processors must discriminate. For this situation, the sensor cross- 
coherence matrix is

$$
\overline{\underline{S}}_{\mathrm{N}}=\overline{\underline{\mathbf{s}}}_{\mathrm{n}}+|\alpha|^{2} \underline{\underline{\mathbf{E}}} \underline{\underline{E}}^{+} \text {. }
$$

After much algebra, it can be shown that

$$
\begin{aligned}
& \underline{\underline{\underline{S}}}_{M}=\left(\overline{\underline{E}}_{M}^{+} \overline{\underline{s}}_{n}^{-1} \overline{\underline{E}}_{M}\right)^{-1}+
\end{aligned}
$$

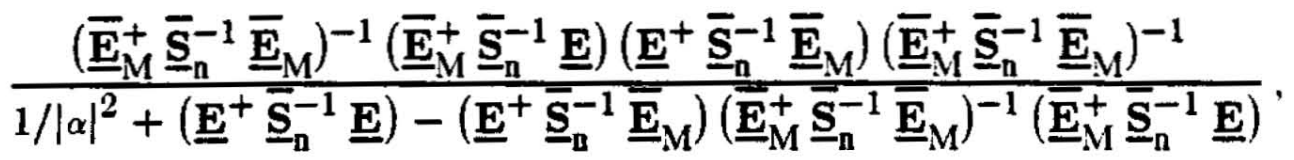

while

$$
\begin{aligned}
& \underline{\hat{\mathbf{S}}}_{\mathrm{M}}^{\prime}=\left(\overline{\underline{\mathbf{E}}}_{\mathrm{M}}^{+} \overline{\mathbf{S}}_{\mathrm{n}}^{-1} \overline{\underline{\mathbf{E}}}_{\mathrm{M}}\right)^{-1}+
\end{aligned}
$$

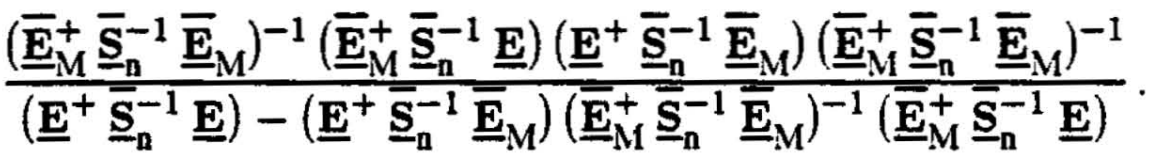

Note that the result in equation (B.14) is completely independent of the amplitude of the $M+1^{\text {st }}$ mode, and is in fact identical in form to equation (B.10). This is indicative of the fact that the beamformer containing $M+1$ modes automatically generates a null in the direction of the $M+1^{\text {st }}$ mode for the beam patterns of all the other modes, whether or not any signal is actually present there. The result in equation (B.13), on the other hand, balances the amount of noise against the amount of $M+1^{\text {st }}$ mode signal, optimally deploying the extra null to best discriminate against the combination of the two. When the amplitude goes to zero, the second term in equation (B.13) disappears entirely, and the beamformer completely ignores the $M+1^{\text {st }}$ mode. Conversely, as the amplitude gets larger, the beamformer increasingly discriminates against the mode; at very large amplitudes. it is forced to devote a full null to the mode, and result approaches that of equation (B.14). 
If a multiple beam MLM beamformer containing $M$ modes always produces better performance than one containing $M+1$ modes, then a $M-1$ mode beamformer always performs better than one of $M$ modes. Thus, it is obvious that a single beam MLM beamformer (which contains only one mode) always provides better performance than any multiple beam MLM beamformer. However, this statement must be qualified by the assumptions inherent in the derivation of the MLM algorithm, particularly the assumption that the signal and noise be independent of each other. For a single beam MLM beamformer operating in a sound field consisting of many modes, this assumption is met only if the different modes are incoherent with respect to each other. If this is not the case, then coherent interference adversely affects the performance of the single beam algorithm, a degradation not accounted for in the analysis presented here. 


\section{Bibliography}

[1] K. Aki and P.G. Richards. Quantitative Seismology: Theory and Meihods. W.H. Freeman and Company, San Francisco, 1980.

[2] A.B. Baggeroer. Space/Time Random Processes and Optimum Array Processing. Technical Report TP 506, NUC San Diego, 1976.

[3] A.B. Baggeroer (private correspondence).

[4] A.B. Baggeroer. A Numerical Approach to the Solution of Acoustic Wave Equations (unpublished).

[5] A. Beillis and F.D. Tappert. Coupled Mode Analysis of Multiple Rough Surface Scattering. Journal of the Acoustical Society of America 66(3):811-8:20. 1979.

[6] C.M. Bender and S.A. Orszag. Advanced Mathematical Methods for Scientists and Engineers. McGraw-Hill Book Company, New York, 1978.

[7] L.M. Brekhovshikh. Waves in Layered Media. Academic Press, New York, 1980.

[8] L.M. Brekhovshikh and Y. Lysanov. Fundamentals of Ocean Acoustics. Springer-Verlag, Berlin, 1982.

[9] H.P. Bucker. Use of Calculated Sound Fields and Matched-Field Detection to Locate Sound Sources in Shallow Water. Journal of the Acoustical Society of America 59(2):368-373, 1976.

[10] J. Capon. High-Resolution Frequency-Wavenumber Spectrum Analvsis. Proceedings of the IEEE 57(8):1408-1418, 1969.

[11] J. Capon and N.R. Goodman. Probability Distributions for Estimators of the Frequency-Wavenumber Spectrum. Proceedings of the IEEE 58(10):1785-1786. 1970 .

[12] B. Carahan, W.A. Luther, and J.O. Wilkes. Applied Numerical Methods. John Wiley \& Sons, New York, 1969.

[13] G.C. Carter, C.H. Napp, and A.H. Nuttall. Statistics of the Estimate of the Magnitude-Coherence Function. IEEE Transactions of Audio and Electroacoustics 21(4):388-389, 1973.

[14] General Bathymetric Chart of the Oceans 5.17, 5th ed., Aug, 1979. 
[15] Y.M. Chen. Report for a Study on Sound Speed Profiles of the Arctic Ocean. Technical Report, MIT Department of Ocean Engineering, 1981.

[16] Y.M. Chen. Underwater Acoustic Ambient Noise in the Arctic. Master's thesis, Massachusetts Institute of Technology, 1982.

[17] C.S. Clay. Waveguides, Arrays, and Filters. Geophysics 31:501-505, 1966.

[18] C.S. Clay and H. Medwin. Acoustical Oceanography. John Wiley \& Sons, New York, 1977.

[19] L.K. Coachman and K. Aagard. Physical Oceanography of Arctic and SubArctic Seas. Marine Geology and Oceanography of the Arctic Seas. SpringerVerlag, Berlin, 1974.

[20] R. Courant and D. Hilbert. Methods of Mathematical Physics. John Wiley \& Sons, New York, 1937.

[21] H. Cox. Resolving Power and Sensitivity to Mismatch of Optimum Arrav Processors. Journal of the Acoustical Society of America 54(3):771-785, 1973.

[22] R.L. Deavenport and F.R. DiNapoli. Evaluation of Arctic Transmission Loss Models. Technical Report TM 821106A, NUSC New London, 1982.

[23] F.R. DiNapoli, D. Viccione, and H. Kutschale. The Arctic Channel: An Acoustic Waveguide. Technical Report TM 781078, NUSC New London, 1978.

[24] F.R. DiNapoli, R. Nielsen, D. Potter, and P.L. Stocklin. TRISTEN/FR.4M IV CW Spatial Coherence and Temporal Stability. Technical Report TD 7095, NUSC New London, 1984.

[25] F.R. DiNapoli. Low Frequency Attenuation in the Arctic Ocean. Technical Report TM 851130, NUSC New London, 1985.

[26] F.R. DiNapoli (private correspondence).

[27] L.B. Dozier and F.D. Tappert. Statistics of Normal Node Amplitudes in il Random Ocean. I. Theory. Journal of the Acoustical Society of America 63(2):353-365, 1978.

[28] L.B. Dozier and F.D. Tappert. Statistics of Normal Mode Amplitudes in a Random Ocean. II. Computations. Journal of the Acoustical Society of America 64(2):533-547, 1978.

[29] G.L. Duckworth. Processing and Inversion of Arctic Ocean Refraction Data. PhD thesis, Massachusetts Institute of Technology, 1983.

[30] G.L. Duckworth and A.B. Baggeroer. Inversion of Refraction Data from the Fram and Nansen Basins of the Arctic Ocean. Tectonophysics 114:5.5-102, 198.;. 
[31] I. Dyer. Statistics of Sound Propagation in the Ocean. Journal of the Acoustical Society of America 48(1):337-345, 1970.

[32] I. Dyer. The Song of Sea Ice and Other Arctic Melodies. Arctic Technology and Policy. Hemishpere Publishing Corporation, Washington, 1984, pages 11-37.

[33] R.H. Ferris. Comparison of Measured and Calculated Normal Mode Amplitude Functions for Acoustic Waves in Shallow Water. Journal of the Acoustical Society of America 52(3):981-988, 1972.

[34] R.G. Fizell and S. Wales. Source Localization in Range and Depth in an Arctic Enviroment. Journal of the Acoustical Society of America 78(S1):S57-S58, 1985. (Abstract only).

[35] G.D. Garland. Introduction to Geophysics. W.B. Saunders Company, Philadelphia, PA, 1979.

[36] G.H. Golub and C.F. Van Loan. Matrix Computations. John Hopkins University Press, Baltimore, MD, 1983.

[37] K.F. Graff. Wave Motion in Elastic Solids. Ohio State University Press, 1975.

[38] S.P. Hayes, P. Ripa, and L.J. Mangum. On Resolving Vertical Modes with Observational Data. Journal of Geophysical Research 90(C4):7227-7234, 1985.

[39] R.M. Heitmeyer, W.B. Moseley, and R.G. Fizell. Full Field Ambiguitv Function Processing in a Complex Shallow Water Environment. In R.A. Wagstaff and A.B. Baggeroer (editors), High-Resolution Spatial Processing in Linderuater Acoustics, pages 171-192. NORDA, NSTL, Mississippi, 1983.

[40] F.B. Hildebrand. Methods of Applied Mathematics. Prentice-Hall, Inc., Englewood Cliffs, NJ, 1965.

[41] F.B. Hildebrand. Advanced Calculus for Applications. Prentice-Hall. Inc.. Englewood Cliffs, NJ, 1976.

[42] M.J. Hinich. Maximum Liklihood Signal Processing for a Vertical Array. Journal of the Acoustical Society of America 54(2):499-503, 1973.

[43] M.J. Hinich. Array Design for Measuring Source Depth in a Horizontal Waveguide. SIAM Journal of Applied Mathematics 32(4):800-806, 1977.

[44] H. Hobaek, C.T. Tindle, and T.G. Muir. Model Experiments on Normal Mode Propagation in a Wedge. Journal of the Acoustical Society of America 78(S1):S70, 1985. (Abstract only). 
[45] D.H. Johnson and S.R. DeGraaf. Improving the Resolution of Bearing in Passive Sonar Arrays by Eigenvalue Analvsis. IEEE Transactions on Acoustics. Speech, and Signal Processing 30(4):638-647, 1982.

[46] W.A. Kuperman. Coherent Component of Specular Reflection and Transmission at a Randomly Rough Two-Fluid Interface. Journal of the Acoustical Society of America 58(2):365-379, 1975.

[47] W.A. Kuperman and F. Ingenito. Attenuation of the Coherent Component of Sound Propagating in Shallow Water with Rough Boundaries. Journal of the Acoustical Society of America 61(5):1178-1187, 1977.

[48] H.W. Kutschale and T. Lee. Bottom-Interacting Acoustic Signals in the Arctic Channel: Long-Range Propagation. Journal of the Acoustical Society of America 74(S1):S1, 1983. (Abstract only).

[49] L. Lawson and R.J. Hansen. Solving Least Squares Problems. Prentice-Hall. Inc., Englewood Cliffs, NJ, 1974.

[50] N.C. Makris and I. Dyer. Environmental Correlates of Pack Ice Noise. Journal of the Acoustical Society of America 79(5):1434-1440, 1986.

[51] H.W. Marsh and R.H. Mellen. Underwater Sound Propagation in the Arctic Ocean. Journal of the Acoustical Society of America 35(4):552-563, 1963.

[52] R.H. Mellen and H.W. Marsh. Underwater Sound Reverberation in the Arctic Ocean. Journal of the Acoustical Society of America 35(10):1615-16-18. 1963.

[53] R.H. Mellen. Underwater Acoustic Scattering from Arctic Ice. Journal of the Acoustical Society of America 40(5):1200-1202, 1966.

[54] R.H. Mellen. Ray-Mode Equivalence in the Arctic Sound Channel. Journal of the Acoustical Society of America 74(S1):S2, 1983. (Abstract only).

[55] R.H. Mellen and F.R. DiNapoli. Underwater Acoustics in the Arctic Ocean. In Proceedings of the Con ference on Methods in Underwater Acoustics. N.ITO Advanced Study Institute, Luneberg, Germany, 1984.

[56] P.N. Mikhalevsky. The Statistics of Finite Bandwidth, Modulated .tcoustic Signals Propagated to Long Ranges in the Ocean, Including Multiple Source Effects. PhD thesis, Massachusetts Institute of Technology, 1979.

[57] P.N. Mikhalevsky. Characteristies of CW Signals Propagated under the Ice in the Arctic. Journal of the Acoustical Society of America $70(6): 1717-1722,1981$.

[58] D.M. Milder. Rav and Mode Invariants for SOFAR Channel Propagation. Journal of the Acoustical Society of America 46(6):1259-1263, 1969. 
[59] A.R. Milne. Sound Propagation and Ambient Noise Under Ice. Underwater Acoustics. Pleneum Press, New York, 1967, Chapter 7.

[60] P.M. Morse and H. Feshbach. Methods of Theoretical Physics (Parts I and II). McGraw-Hill Book Company, New York, 1953.

[61] J.N. Newman. Marine Hydrodynamics. The MIT Press, Cambridge, MA, 1977.

[62] A.V. Oppenheim and R.W. Schafer. Digital Signal Processing. PrenticeHall, Inc., Englewood Cliffs, New Jersey, 1975.

[63] T.W. Parks and J.H. McClellan. A Program for the Design of Linear Phase Finite Impulse Response Filters. IEEE Transactions of Audio and Electroacoustics 20(4):280-288, 1972.

[64] C.L. Pekeris. Theory of Propagation of Explosive Sound in Shallow Water. Geological Society of America, Memoir 27, 1948.

[65] A.D. Pierce. Extension of the Method of Normal Modes to Sound Propagation in an Almost-Stratified Medium. Journal of the Acoustical Society of America 37(1):19-27, 1965.

[66] J.G. Pierce and K. Hickox. A Review of the Soviet Open Literature on Acoustic Research in the Arctic. Technical Report KFR 423-83, Ketron, Inc., Arlington, VA., 1983.

[67] J.J. Polcari. Low Frequency Acoustic Signal Propagation in the Arctic Ocean. Master's thesis, Massachusetts Institute of Technology, 1983.

[68] J.J. Polcari. An Array-Processor Based Time Series Processing System (internal software documentation).

[69] M.B. Porter (private correspondence).

[70] M.B. Porter, R.L. Dicus, and R.G. Fizell. Simulations of Matched-Field Processing in a Deep-Water Pacific Environment (in preparation).

[71] K. Prada, K. Vonderheydt, and T.F. O'Brien. A Versatile Multichannel Data Acquisition System for Seismic and Acoustic Application. In Proceedings of the IEEE Con ference Oceans '81, pages 44-47. 1981.

[72] S.R. Rutherford. An Examination of Coupled Mode Theory as Applied to Underwater Sound Propagation. PhD thesis, University of Texas at Austin, 1979.

[73] E.K. Scheer. Estimates of Crustal Transmission Losses Using MLM Array Processing. Master's thesis, Massachusetts Institute of Technology, 1982. 
[74] F.C. Schweppe. Sensor-Array Data Processing for Multiple-Signal Sources. IEEE Transactions on In formation Theory 14(2):294-305, 1968.

[75] E.C. Shang, C.S. Clay, and Y.Y. Wang. Passive Harmonic Source Ranging in Waveguides by Using Mode Filter. Journal of the Acoustical Society of America 78(1):172-175, 1985.

[76] E.C. Shang, L.M. Lawson, and D.R. Palmer. Source Range Information Loss in Waveguides. Journal of the Acoustical Society of America 79(4):958-963, 1986.

[77] G.W. Sheppard. Arctic Ocean Ambient Noise. Master's thesis, Massachusetts Institute of Technology, 1979.

[78] W. Tiemann, J. Ardai, B. Allen, and T.O. Manley. Geophysical Data from the Drifting Ice Stations FRAM IV and TRISTEN. Technical Report LDGO-82-3, LDGO, Columbia University, Palisades, NY, 1982.

[79] I. Tolstoy and C.S. Clay. Ocean Acoustics. McGraw-Hill Book Company, New York, 1966.

[80] R.J. Urick. Principles of Underwater Sound. McGraw-Hill Book Company, New York, 1975.

[81] H.L. Van Trees. Dectection, Estimation, and Modulation Theory. Part I. Detection, Estimation, and Linear Modulation Theory. John Wiley \& Sons, New York, 1968.

[82] H.L. Van Trees. Dectection, Estimation, and Modulation Theory. Part III. Radar-Sonar Signal Processing and Gaussian Signals in Noise. John Wiley \& Sons, New York, 1971.

[83] P. Wadhams. Arctic Sea Ice Morphology and Its Measurement. Arctic Technology and Policy. Hemishpere Publishing Corporation, Washington, 198.1. pages 11-37.

[84] T.C. Yang and G.R. Giellis. Application of High-Resolution Technigues to Vertical Array Beamforming in the Arctic Ocean. In R.A. Wagstaff and A.B. Baggeroer (editors), High-Resolution Spatial Processing in Underwater Acoustics. pages 171-192. NORDA, NSTL, Mississippi, 1983.

[85] T.C. Yang (private correspondence). 


\section{Biography}

Lieutenant John Polcari, USN, graduated from the U.S. Naval Academy in June, 1977, receiving a B.S. degree in Electrical Engineering. After graduation and subsequent commissioning in the U.S. Navy, he served a two year tour of duty in the weapons department of the USS John C. Calhoun (SSBN 630 Gold), where he completed his submarine qualification requirements. After completion of his duty on the Calhoun, he was transferred to the professional engineering corps of the U.S. Navy and ordered to post-graduate school in the Naval Construction and Engineering curriculum at the Massachusetts Institute of Technology in June, 1980. While at MIT, he completed a thesis entitled "Low Frequency Acoustic Signal Propagation in the Arctic Ocean", and was awarded the dual degrees of M.S. in Electrical Engineering and Ocean Engineer in June, 1983. At that time, he was awarded the Naval Sea Systems Command Award in Naval Construction and Engineering for his performance during the course of instruction. He has since continued at MIT in pursuit of an Sc.D. in Oceanographic Engineering in the Joint Program between MIT and Wood's Hole Oceanographic Institute. His primary technical interests include military applications of signal processing techniques 10 the field of underwater acoustics, particularly with respect to arrays and other spatial issues. 